\title{
The nature and applicability of the blackboard architecture
}

Citation for published version (APA):

Velthuijsen, H. (1992). The nature and applicability of the blackboard architecture. [Doctoral Thesis, Maastricht University]. Rijksuniversiteit Limburg. https://doi.org/10.26481/dis.19920924hv

Document status and date:

Published: 01/01/1992

DOI:

10.26481/dis.19920924hv

Document Version:

Publisher's PDF, also known as Version of record

\section{Please check the document version of this publication:}

- A submitted manuscript is the version of the article upon submission and before peer-review. There can be important differences between the submitted version and the official published version of record.

People interested in the research are advised to contact the author for the final version of the publication, or visit the DOI to the publisher's website.

- The final author version and the galley proof are versions of the publication after peer review.

- The final published version features the final layout of the paper including the volume, issue and page numbers.

Link to publication

\footnotetext{
General rights rights.

- You may freely distribute the URL identifying the publication in the public portal. please follow below link for the End User Agreement:

www.umlib.nl/taverne-license

Take down policy

If you believe that this document breaches copyright please contact us at:

repository@maastrichtuniversity.nl

providing details and we will investigate your claim.
}

Copyright and moral rights for the publications made accessible in the public portal are retained by the authors and/or other copyright owners and it is a condition of accessing publications that users recognise and abide by the legal requirements associated with these

- Users may download and print one copy of any publication from the public portal for the purpose of private study or research.

- You may not further distribute the material or use it for any profit-making activity or commercial gain

If the publication is distributed under the terms of Article $25 \mathrm{fa}$ of the Dutch Copyright Act, indicated by the "Taverne" license above, 


\section{The Nature and Applicability of the Blackboard Architecture}

\section{PROEFSCHRIFT}

ter verkrijging van de graad van doctor aan de Rijksuniversiteit Limburg te Maastricht, op gezag van de Rector Magnificus, Prof. Mr. M.J. Cohen, volgens het besluit van het College van Dekanen, in het openbaar te verdedigen op donderdag, 24 september 1992 om 16.00 uur

door

Hugo Velthuijsen 


\section{CIP-GEGEVENS KONINKLIJKE BIBLIOTHEEK, DEN HAAG}

Velthuijsen, $\mathrm{H}_{\text {. }}$

The nature and applicability of the blackboard

architecture / $H$. Velthuijsen

- Leidschendam: PTT Research - III.

Proefschrilt Maastricht - Met index, lit. opg.

ISBN 90-72॥25-34-7

Trefl $w_{:}$expertsystemen.

Graphic design: PTT Research, General affairs

(C) 1992 by PTT Research

Subject to the expectations provided for by law, no parts of this publication may be reproduced and/or published in prine, by photocopying on microfilm or in any other way without the written consent of the copyright owner. The same applies to whole or partial adaptations. The copyright owner retains the sole right to collect from third parties fees payable in respect of copying and/or to take legal or other action for this purpose. 


\section{promotor:}

Prof. dr. H.J. van den Herik

\section{co-promotor:}

Dr. P.J. Braspenning

beoordelingscommissie:

Prof. dr. ir. A. Hasman (voorzitter)

Prof. dr. ir. E. Backer (Technische Universiteit Delft)

Dr I.D. Alexander-Craig (University of Warwick)

Prof. dr. G.R. Renardel de Lavalette (Rijksuniversiteit Groningen)

Prof. dr. ir. drs. O.J. Vrieze

Dit proefschrift is tot stand gekomen mede dankzij de ondersteuning van PTT Research. 
8 


\section{Acknowledgements}

First of all, I am grateful to my supervisors at the University of Limburg for their support. I thank Jaap van den Herik for giving me the opportunity to pursue my Ph.D. degree at the University of Limburg, and for his advice and suggestions. I want to thank Peter Braspenning for his guidance during the realization of this thesis and for helping me on my way to becoming a scientist.

The research reported on in this thesis has been carried out at PTT Research, the research laboratories of the Royal PTT Nederland NV. I am grateful to the Managing Board of PTT Research for providing the means for the work leading to this thesis. Particularly, I wish to thank Michel Antal, General Director of PTT Research, who funded the research, and Harry van Binsbergen, former Head of the Computer Science Department and current Director of Tele-Informatics at PTT Research, who actively supported my request to pursue a $\mathrm{Ph} . \mathrm{D}$. degree.

The work reported in this thesis builds on and extends research carried ont by a number of project groups. I am indebted to my fellow members of these groups and am grateful for their contributions to the work that forms a basis for this thesis: Jeanette Vonk, Ben Lippolt, Gidi van Liempd, and Adriana Florescu.

I also wish to thank Mariette Legtenberg and Radjen Kishna, who implemented parts of the systems described in this thesis during their traineeships at PTT Research.

Special thanks go to Ben Lippolt, Gidi van Liempd, and Martin Kooij for proofreading earlier versions of the thesis and for providing me with a knowledgeable environment in which I could discuss my ideas. Furthermore, I wish to thank Iain Alexander-Craig for his careful proofreading 
and many helpful suggestions.

Finally, I wish to thank Han Zuidweg, Kees Middelburg, and Bart Nieuwenhuis, who blazed the trail that II followed.

Hugo Velthuijsen Morristown, July 1992. 


\section{Contents}

1 Introduction 1

1.1 Scope of the thesis and terminology ......... 4

1.2 Problem definition and issues addressed $\ldots \ldots \ldots 6$

1.3 Structure of the thesis ............. 7

2 The Blackboard Architecture 9

2.1 Blackboard ................... 10

2.2 Knowledge sources . . . . . . . . . . . 17

2.3 Control . . . . . . . . . . . . . . 20

2.4 Concurrent blackboard architectures . . . . . . . 26

2.5 Cognitive models $\ldots \ldots \ldots \ldots \ldots \ldots \ldots \ldots$

2.6 Blackboard applications . . . . . . . . . . 38

2.7 Blackboard frameworks and generators . . . . . . . 48

3 The Blackboard Framework Blondie 53

3.1 Blondie ........................ 55

3.2 Evaluation . . . . . . . . . . . . . 72 
4 A Parallel Blackboard Framework for Robot Control $\quad 79$

4.1 The application - robot control ......... 80

4.2 Parallelisation of Blondie ............. 85

4.3 Related work . . . . . . . . . . . . . . 99

4.4 Evaluation . . . . . . . . . . . . . . . 101

5 The Distributed Blackboard Framework Blondie-III 105

5.1 Blondie-III . . . . . . . . . . . . 107

5.2 Communications . . . . . . . . . . . 118

5.3 Related work . . . . . . . . . . . . 125

5.4 A configuration task for a telecommunication network . . 126

5.5 Network-configuration implementation . . . . . . . 128

5.6 Evaluation . . . . . . . . . . . . . . . 139

6 Formal Description of Blackboard Architectures 143

6.1 Choice of formalism . . . . . . . . . . . . 144

6.2 The blackboard metaphor . . . . . . . . . . 146

6.3 Blackboard system . . . . . . . . . . . . . 149

6.4 Control unit . . . . . . . . . . . . . . . 154

6.5 Blackboard . . . . . . . . . . . . . 162

6.6 Knowledge sources . . . . . . . . . . . 166

6.7 Related work . . . . . . . . . . . . . 175

6.8 Evaluation . . . . . . . . . . . . 176

$\begin{array}{lll}7 & \text { Options and Variations } & 179\end{array}$

7.1 Existing options and variations . . . . . . . . . 182

7.2 Extending the existing options and variations . . . . . 198

7.3 Distinctive features of the blackboard architecture . . . 219

7.4 Evaluation . . . . . . . . . . . . . 223 
8 A Proposal for Integration of Goal-directed Control 225

8.1 Goal-directed control in blackboard architectures . . . . 2227

8.2 Maes' spreading activation network . . . . . . . 234

8.3 A blackboard activation network . . . . . . . . 249

8.4 Evaluation . . . . . . . . . . . . . . . 259

9 Conclusions

9.1 The nature of the blackboard architecture . . . . . . 262

9.2 Applicability of the blackboard architecture . . . . . . 264

9.3 Future methodological work . . . . . . . . . 267

Bibliography

A Formal Description of Blackboard Architectures $\quad 295$

A.1 Hearsay-II . . . . . . . . . . . . . . . . . . 296

A.2 Hearsay-III . . . . . . . . . . . . . 300

A.3 DVMT . . . . . . . . . . . . . . . . . . 304

A.4 HASP/SIAP $\ldots \ldots \ldots \ldots \ldots \ldots \ldots$

A.5 AGE . . . . . . . . . . . . . . . . 312

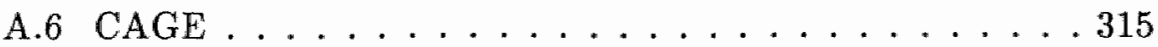

A.7 POLIGON $\ldots \ldots \ldots \ldots \ldots \ldots \ldots$

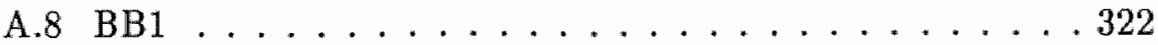

A.9 AGORA . . . . . . . . . . . . . . . . 327

A.10 GBB . . . . . . . . . . . . . . . . . . . . .

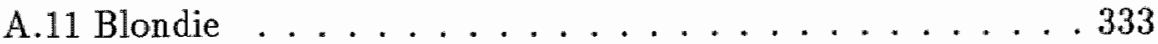

A.12 Blondie-II . . . . . . . . . . . . . . . 337

A.13 Blondie-III . . . . . . . . . . . . . . . . . 342

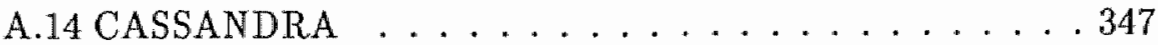

Index 
Summary

359

Samenvatting

363

Curriculum Vitae

369 


\section{List of Figures}

1.1 The blackboard metaphor. . . . . . . . . . . . 2

1.2 The blackboard architecture; basic components. . . . . . 2

2.1 Blackboard levels in Hearsay-II. . . . . . . . . . . 13

2.2 Blackboard panels in OPM. . . . . . . . . 14

2.3 Options in communication. . . . . . . . . 15

2.4 Example of a control loop. . . . . . . . . . . 21

2.5 Simplified illustration of the interactive activation model. 35

3.1 Description of the number game. . . . . . . . 54

3.2 Example of a blackboard unit. . . . . . . . . 56

3.3 Panels and levels in the test application. . . . . . . 57

3.4 Example of the control blackboard panel. . . . . . . 58

3.5 Example of the domain blackboard panel. . . . . . . 59

3.6 Example of an event. . . . . . . . . . . 61

3.7 Example of a KS descriptor. . . . . . . . . . . 63

3.8 Example of a Knowledge Source Activation Record (KSAR). 64

3.9 KS interactions in the test application. . . . . . . 67

3.10 Module structure in Blondie. . . . . . . . . . . . 71

4.1 The experimental robot cell at PTT Research. . . . . 81

4.2 A "flexible" robot cell architecture. . . . . . . . 83 
4.3 Supervisor interaction. . . . . . . . . . . 84

4.4 All virtual processors on one physical processor. . . . . . 89

4.5 Virtual processors distributed over physical processors. . 89

4.6 Mapping between KSs and physical processors. . . . . . 91

4.7 Communication in Blondie-II. . . . . . . . . . . . 93

4.8 Example of a KS descriptor in Blondie-II. . . . . . . 95

4.9 Control loop for parallel KS execution. . . . . . . . . . 96

5.1 Example of a distributed blackboard architecture. . . . 107

5.2 Example of the tree structure used for processing events. . 118

5.3 Overview of communication primitives in Blondie-III. . . 122

5.4 Structure of unit on the blackboard-level communications. 123

5.5 Topology of an imaginary district. . . . . . . . 127

5.6 Communications in the distributed system. . . . . . 130

5.7 Finding a solution on the blackboard. . . . . . . 134

5.8 The flow diagram of select- KSs. . . . . . . 135

5.9 Example of a rule in the configuration application. . . 138

6.1 Merging the KS cycles into one control cycle. . . . . 156

6.2 Control cycles in a distributed-control blackboard architecture. . . . . . . . . . . . . . 161

6.3 Control cycle in a parallel blacliboard architecture. . . . 162

6.4 Structure of discussion. . . . . . . . . . 163

6.5 States and transitions for a KS instantiation. . . . . 174

7.1 A map of existing options and variations. . . . . . 181

7.2 Variations in information used for selection of activities. . 211

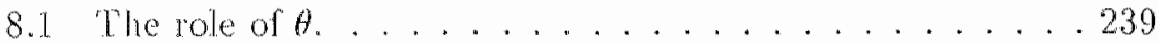

8.2 The role of $\sigma, \gamma$ and $\delta . \ldots \ldots . \ldots 240$ 
8.3 Bias towards ongoing sequences of actions. . . . . . 241

8.4 Activation spreading in a network. . . . . . . . 246

8.5 An activation network when KSIs would be included. . . . 251

8.6 Procedures needed for matching and unification. . . . . 253

8.7 Preselection of KSIs for participation in the KSI selection by an activation network. . . . . . . . . 257 
viii

List of Figures 


\section{List of Tables}

2.1 Parallel blackboard architectures. . . . . . . . . . 27

2.2 Distributed blackboard architectures. . . . . . . . . 31

2.3 Blackboard-application categories. . . . . . . . 38

2.4 Interpretation applications. . . . . . . . . . . 39

2.5 Monitoring over time applications. . . . . . . . 41

2.6 Planning and configuration applications. . . . . . 43

2.7 Control applications. . . . . . . . . . . 45

2.8 Integration applications. . . . . . . . . 47

2.9 Frameworks and generators. . . . . . . . . . . 49

3.1 Knowledge sources in the test application. . . . . . 66

6.1 Notation for dynamic aspects. . . . . . . . . 147

6.2 Notation for static aspects. . . . . . . . . . 148

7.1 Overview of formally described black board architectures (apart from Blondie, Blondie-II, and Blondie-III). . . . 180

7.2 Variation observed in Hearsay-II, Hearsay-III, and DVMT. 184

7.3 Variation observed in HASP/STAP, AGE, CAGE, and POLIGON. . . . . . . . . . . . . . 185

7.4 Variation observed in BB1, AGORA, and GBB..... 186

7.5 Variation observed in Blondie, Blondie-II, Mlondie-III and

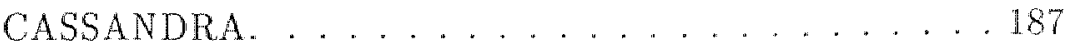


7.6 Summary of options observed in the fourteen blackboard architectures. . . . . . . . . . . . . . 188

8.1 Goal relationships . . . . . . . . . . . . 227

8.2 Glossary of notation in Maes' activation network. . . . . . 243

8.3 Blackboard systems components used for implementing elements of an activation network. . . . . . . . . . 249 


\section{Chapter 1}

\section{Introduction}

The blacliboard architecture is a knowledge-based system architecture first suggested in (Newell, 1962) and first implemented in the early seventies as the architectural framework for the speech-understanding system Hearsay-II (Lesser et al, 1975). The blackboard architecture has later been applied in many other problem domains, such as interpretation of sensor data, planning, control of systems and processes, and medical diagnosis, illustrating the power of the architecture.

The blackboard architecture can be illustrated best by the metaphor that lent it its name. Imagine several specialists with different expertise required to solve a problem cooperatively. The specialists are gathered around a blackboard for communicating intermediate results and hypotheses (see Figure 1.1). As soon as data has been written on the blackboard by one of the specialists, other specialists may decide that they can use that information to contribute to the problem-solving process. These specialists in turn write their own findings on the blackboard, thus providing possibly interesting information for other specialists to continue the problem-solving process. The process so continues until either a solution to the problem is found or no specialist is able to proceed on any of the data present on the blackboard.

The blackboard architecture models this type of problem-solving behaviour (see Figure 1.2). Separate, otherwise independent computer programs. called knowledge sources (KSs), are given the role of specialists. A global data structure (the blackboard) is used to write and 


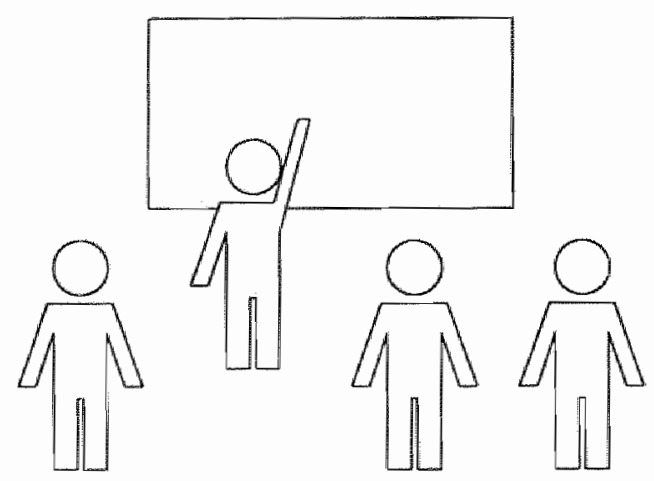

Figure 1.1: The blackboard metaphor: specialists gathered around a blackboard for communicating intermediate results and hypotheses.

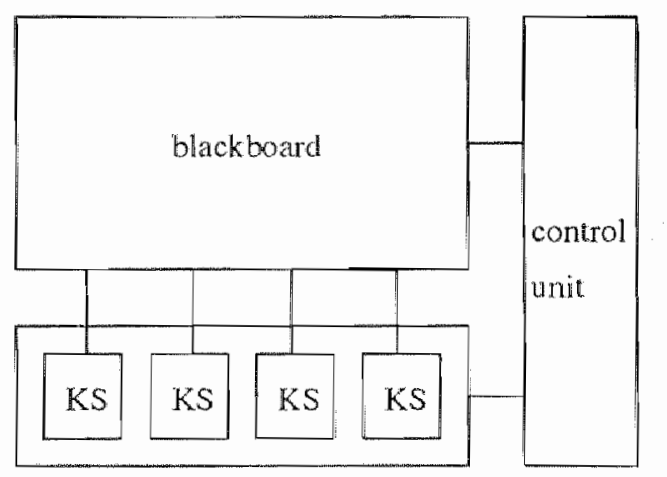

Figure 1.2: The blackboard alchitecture; basic components.

read intermediate results and hypotheses. Knowledge sources communicate only via the blackboard. In many blackboard systems a control unit is added. The control unit mediates between competing knowledge sources. For this tasls it can use information on KSs and data stored on the blackboard. Metaphorically, a control unit is given the role of discussion leader.

Some characteristics of the blackboard architecture are immediately apparent from this description. The blacliboard architecture is strictly modular because of the subdivision in knowledge sources. This subdi- 
vision can be used to incorporate different processing (and reasoning) methods, such as procedural and rule-based processing or numeric and symbolic processing, within one system.

A blackboard serves two distinct roles. Firstly, communication via a global data structure enables asynchronous communication between modules. A knowledge source does not need to know addresses or possible recipients of messages; interested parties monitor the blackboard for relevant information themselves. Secondly, information present on the blackboard forms a representation of the current state of the problemsolving process. This source of information about the problem-solving state can be useful not only for determining which knowledge sources are applicable at any given moment, but also for deciding which of the pending activities is most promising, thus enabling sophisticated selection of activities and well-informed problem-solving behaviour.

The blackboard architecture provides a flexible architecture, not only with respect to the specific modules used, but also with respect to the applied activity selection scheme. A system with a blackboard architecture is basically data driven. It detects and exploits opportunities for system activities and does not necessarily proceed in a structured manner, as in a top-down or bottom-up processing, although blackboard systems can be implemented to do so. Selection of activities in a blackboard architecture can be made to proceed even in a goal-directed manner, as has been shown in (Corkill et al., 1982; Lesser et al, 1989b).

At first glance, some characteristics of the blackboard architecture make the architecture particularly suited for concurrent processing. "The strict subdivision into more or less independent programs within the architecture suggests opportunities for pluysical distribution. The presence of the blackboard provides a means for communication, no matter what the physical location of components might be. Several examples exist of blackboard architectures, where knowledge sources, the control component, and even the blackboard have been distributed in a natural way over different processors. A blackboard architecture which allows concurrent execution of knowledge sources is called a parallel blackboard architecture, provided that the blackboard and control unit are not distributed. A distributed blackboard architecture is a blackboard architecture with a distributed blackboard and control unit. 


\subsection{Scope of the thesis and terminology}

Artificial as a science has basically two different approaches that motivate research; the cognitive science approach and the engineering approach. The cognitive science approach deals with building models of human behaviour and validating these models by studying computer programs based on these same models. The engineering approach attempts to create computer programs to perform tasks at which, currently, humans are better. Here, human performance may provide examples and inspiration, but is not considered as a strict guideline. In practice, actual motivation for research can lie between these two extremes. The engineering approach is most significant in industrial development and applied research.

Similarly, the blackboard architecture is an important architecture in two different respects. The blackboard architecture provides a model and implementation of opportunistic problem solving, which has been proposed as a cognitive model for errand-planning tasks (Hayes-Roth \& Hayes-Roth, 1979) and for recognising printed words (McClelland \& Rumelhart, 1981; Rumelhart \& McClelland, 1982)1. Aspects of the blackboard architecture, however, have been applied also successfully in situations, where human behaviour did not necessarily provide the norm.

Currently, two different views on the blackboard architecture still exist. In the cognitive view the blackboard architecture is a model of problem solving. The definition of the architecture in that view necessitates an abstraction hierarchy on the blacliboard, knowledge sources that operate on one level of abstraction of the blackboard and post results on another level, and opportunistic selection of knowledge sources. This is the definition as can be found in ( $\mathrm{Nii}, 1986 \mathrm{a} ; \mathrm{Nii}, 1986 \mathrm{~b}$ ).

The engineering view presupposes only a global, organised data structure for communication among otherwise independent chunks of computer program. It uses adcitional elements and characteristics from the full blackboard model of problem solving according to the demands of the application domain.

\footnotetext{
'Both models and how they compare to the blackboard architecture will be disucsed in Chapter 2.
} 
Existing blackboard architectures can be found between these two extremes, where at one end we find systems using a blackboard solely as a means for asynchronous communication (see also Winston, 1984, ch. $5)^{2}$, while at the other end the complete cognitive model can be found. This thesis adopts the engineering view on the blackboard architecture, seeing the blackboard architecture as a useful tool for implementing computer systems in complex domains. We will not deal with how much the blackboard architecture provides indeed a useful model of certain cognitive tasks.

Certain terms, such as 'blackboard model', 'blackboard architecture', and 'blackboard shell', are used extensively throughout the literature as well as in this thesis. As there did not exist consensus on their exact meaning, a proposal for definitions was given in the introduction of (Engelmore \& Morgan, 1988). These definitions are adopted in this thesis. We also define several other terms for the scope of this thesis. This leads to the following terminology (further exact definitions of relevant terms are given in Chapter 6):

problem solving Problem solving is the process of finding a solution to a given problem. We assume that problem solving proceeds in a 'problem space'. The problem space consists of "states". At any time the problem solver is in a certain state, characterised by what has been achieved (and what has not yet been achieved) when the problem solver is in that state. By taking steps in the problemsolving process (the specific nature of these steps is dependent on the domain of the problem solver), the problem solver can nove from one state to another. Usually, certain steps can be taken only when the problem solver is in specific states. Thus, a state also determines indirectly what can be done. The steps comnect stales in the problem space; together they form a graph. The task of a problem solver is to find a path through this graph from the initial state to a state that matches the goals of the problem solver.

blackboard model The blackboard model is a problem-solving morlel. based on observations of human problem solving. The blacklonal

${ }^{2}$ One conld dispute whether such systems should be terned 'blackboard systems" As is argued in Chapter 7 , an essential feature of a blackboard in a blackboard swstom is that it contains, at any time, information about what has been done. whit can ho done now; and what must be done eventually. 
model describes otherwise independent 'chunks of knowledge' used cooperatively to solve a common problem by communicating exclusively via a central data storage. These 'chunks of knowledge' may be embodied for instance by humans or computer programs.

architecture In general, the term 'computer-system architecture' (or just 'architecture') denotes a description of components in that computer system, their interfaces, and their functionality.

blackboard architecture A blackboard architecture is a computersystem architecture implementing the elements as observed in the blackboard model. We use 'the blackboard architecture' as a generic term to indicate all possible blackboard architectures.

blackboard system A blackboard system is a computer system with a blackboard architecture.

blackboard shell $A$ blackboard shell is either a specification of components of a blackboard model or an implementation of such a specification. A blackboard shell is not yet a problem-solving system.

blackboard framework The term 'blackboard framework' is synonymous with 'blackboard shell'.

blackboard application A blackboard application denotes a blackboard system solving problems in a specific problem domain. A blackboard application adds domain-specific elements to a bare blackboard framework to create a 'complete' problem-solving system.

\subsection{Problem definition and issues addressed}

This thesis studies several aspects of the blackboard architecture in detail. The blackboard architecture is a rather complex computational framework, although the blackboard metophor can be explained and itlustrated rather simply. Over the years since the first blackboard system has been implemented it has become clear, that there exist many possible options and variations to choose from for any single implementation 
of the metaphor, while the semantical consequences of particular choices or combinations of choices are not yet sufficiently clear.

In order to be able to apply and exploit the advantages of the blackboard architecture successfully, a deeper understanding of the blackboard architecture is needed. Important questions which must be answered in this respect specifically are the following:

- what distinguishes a blackboard architecture from other computational frameworks and which characteristics make them especially useful for certain applications;

- which options and variations have been used within existing blackboard architectures;

- which (interesting) options and variations and also combinations thereof have not yet been tried; and finally,

- what options and variations lead to what characteristics and how should options be selected for any given application.

The central problem addressed in this thesis is to find answers to these four fundamental questions. In the course of this work several other issues were adciressed as well. These can be listed as follows:

- Applicability of the blackboard architecture in domains relevant within the operations of Koninklijke PTI Nederland NV.

- Exploitation of inherent parallelisn of the blackboard architecture for implementing concurrent blackboard applications.

- Extension of the basically data-driven mode of knowledge-source selection within blackboard architectures with a goal-directed component.

\subsection{Structure of the thesis}

We now present the siructure of this thesis. Chapter 2 contains a more detailed discussion of the blackboard architecture and provides back- 
ground information on the blackboard architecture in general. The emphasis of this discussion is, as hinted in Section 1.1, on the blackboard architecture from an engineering point of view.

We have designed and implemented a number of blackboard frameworks which are presented in the Chapters 3,4 , and 5 . The first of these frameworks is called Blondie ${ }^{3}$ and is described in Chapter 3. Blondie was developed mainly as an instrument to gain practical experience with the blackboard architecture.

Concurrency in blackboard architectures is addressed in the next two chapters. Chapter 4 describes Blondie-II, which is a parallel blackboard framework developed with the domain of robot control in mind. In Blondie-II knowledge sources can be executed in parallel. The third blackboard framework, Blondie-III, is described in Chapter 5. BlondieIII is a distributed blackboard framework, designed for implementing a number of cooperating blackboard systems. Blondie-III has been applied on a telecommunication-network configuration problem, also described in Chapter 5.

Chapters 6 and 7 address the centrall problem of this thesis specifically. Chapter 6 proposes a formal description of blackboard architectures. This formalisation was used to describe a number of well-known and influential blackboard architectures in order to identify existing as well as new and promising options and variations within blackiboard architectures. The actual formal descriptions of these existing blackboard architectures can be found in Appendix A. Results of our formalisation efforts and answers to the four fundamental questions posed in the previous section are presented in Chapter 7 .

Chapter 8 presents a dynamical network-based approach to knowledgesource selection as a proposal for integrating data-directed and goaldirccted knowledge source selection within blackboard systems.

Conclusions to the work presented in this thesis can be found in Chapter 9.

"The name 'Blondie' is a Dutch acronym ("Blackboard Onderzoek in Expert Systemen'), which translates into 'Blackboard Research in Expert. Systems'. 


\section{Chapter 2}

\section{The Blackboard Architecture}

We present the blackboard architecture in more detail in this chapter. This includes discussions in subsequent sections of components which typically constitute a blackboard system, i.e., blackboard, knowledge sources, and control unit. These discussions deal mostly with the indication of important features and assessing what these features can contribute to an application. More detailed descriptions of variations and options for the design of a blackboard architecture, both as observed in existing systems and as conceivable by extending what has been observed, are given in Chapter 7 . Therefore, we refrain from entering into a detailed discussion of variation here as well.

The chapter then proceeds by highlighting the applicability of the blackboard architecture for developing concurrent systems. The next section contains two illustrations of cognitive models based on the blackboard model. We conclude with discussions of existing blackboard applications and frameworks.

Descriptions of the blackboard architecture and of different implementations of the blackboard architecture can be found in (Nii, 1986a; Nii, 1986b), which give a good overview of the state of the art of blackboard technology in 1986. Another useful, more extensive overview is presented in (Engelmore \& Morgan, 1988), in which important papers on the subject are collected. Finally, we refer to (Craig, 1989) for a. 
description of the blackboard architecture, and to (Jagannathan et al., 1989) for a collection of papers on research issues in the area.

\section{$2.1 \quad$ Blackboard}

The blackboard in a blackboard system can be seen as having two distinct functions. The blackboard not only serves as a communications medium between the knowledge sources, but also provides a representation of the current state of the problem-solving process of a blackboard system at any given moment. These different roles, together with a discussion on the structure of the blackboard, are addressed in the following sections.

\subsubsection{Problem-solving state}

The blackboard in a blackboard system contains all information considered to be of relevance to other components within the system, as a. result of interactions by the knowledge sources with the blackboard, In fact, the blackboard may contain information pertaining to previously generated hypotheses, facts, currently held beliefs and views, goals, and relations between data elements. The blackboard thus mirrors the current state of affairs for a problem-solving process and can be used as an indication of what has yet to be done to complete the problem-solving process.

Blackboard systems are able to exploit this explicit representation of the problem-solving state in a number of ways. As data elements can be used in subsequent processing steps, the emergence or existence of data. on the blacliboard may indicate opportunities for future contributions to the problem-solving process by one or more knowledge sources. Correspondingly, each knowledge source in a blackboard architecture has associated with it a specification of the blackboard data or modifications that provide an opportunity for this knowledge source to make a. contribution. In this way the blackboard helps in determining which activities can be executed at each point in time, i.e., it is instrumental in deciding the applicability of knowledge sources. But information on the blackboard can be used also to determine desirability of applying 
a specific knowledge source. This last aspect, however, is a matter of control and is better addressed in the discussion on control issues.

Proposing and subsequently extending and modifying hypotheses leads to the building of solutions from islands (groups of related hypotheses), which is characteristic for many blackboard applications. The blackboard system PROTEAN for determining the structure of proteins uses this feature (called anchors in PROTEAN) explicitly for tying components to specific places in the structure (Hayes-Roth et al, 1986a). The problem-solving process extends a partial solution candidate until it can be reasonably dismissed or until it can be combined with other partial solutions. This way of problem solving can be illustrated by the process of putting together a jigsaw puzzle, as was done in (Nii, 1986a).

An issue often seen as one of the characterising features of the blackboard architecture is its ability to hold competing entries on the blackboard simultaneously and to pursue alternative processing threads at the same time (Nii, 1986b). This is consistent with the idea of viewing the blackboard as containing a representation of the current problemsolving state: possibly competing hypotheses may persist until enough knowledge has been acquired to identify one hypothesis as the correct one. Relations between blackboard elements can be employed to keep track of the dependencies and inference steps.

The blackboard, however, has been applied in blackboard applications in a slightly different manner as well. It has been used to represent the currently held model or interpretation of the 'world' in which the application operated. This approach can be adopted, e.g., in applications to control tasks, such as robot control and process control (see also Section 2.3.2). In such cases the existence of competing entries on the blackboard, representing inconsistent interpretations of the real world, could be undesirable.

The difference between using the blackboard for representing either the current world model or the current problem-solving state is related to the difference between a Truth Maintenance System (TMS, Doyle, 1979) and de Kleer's Assumption-based Truth Maintenance System (ATMS, de Kleer, 1986). On the one hand, a TMS is dedicated to recording and maintaining consistency between derived predicates during a problemsolving process. This mirrors the role of a blackboard representing a current world model. On the other hand, an ATMS keeps track of 
which assumptions (axioms) were used in deriving a truth-value for each predicate and is thus capable of containing conflicting predicates simultaneously. This resembles closely the role of a blackboard representing the current problem-solving state.

\subsubsection{Structure}

According to the blackboard metaphor, knowledge sources write data to and read data from the blackboard. In implementations of the blackboard metaphor data is represented via data structures. The blackboard in most blackboard systems is not just an unorganised collection of data elements, but structured itself to facilitate interaction. The blackboard is essentially different from the working memory in a rule-based expert system, which is normally just an unstructured 'heap' of data. In this section we indicate the advantages of structuring the blackboard and describe some of the usual approaches.

\section{Advantages of structure}

Structuring a blackboard improves accessibility of a blackboard and efficiency in a blackboard system. By grouping together blackboard entries containing 'similar' information, it becomes easier and less laborious to locate and access entries based on the information it should contain. The blackboard framework GBB (Corkill el al., 1986) exploits this opportunity for increasing efficiency of blackboard interactions by supporting several facilities for ordering and structuring data. Structuring also improves understandability of information on the blackboard to users of a blackboard system.

As we have seen in the description of the blackiboard metaphor in the introduction of this thesis, the emergence of data on the blackboard is used to determine whether knowledge sources can contribute to the problemsolving process. Structuring can be used to ensure that all information of interest to a specific knowledge source is grouped together. This way, surveying the blackboard for the emergence of interesting data can be restricted to only a limited area, thus improving efficiency of determining applicability of knowledge sources. 


\begin{tabular}{|l|l|}
\hline interpretations & \\
\hline phrases & \\
\hline word sequences & \\
\hline words & \\
\hline syllables & \\
\hline segments & \\
\hline signals & \\
\hline
\end{tabular}

Figure 2.1: Blackboard levels in Hearsay-II.

\section{Approaches to structuring}

A number of common approaches to structuring the blackboard are now described. The blackboard system Hearsay-II (Erman et al., 1980) subdivided its blackboard into different so called levels which coincided with the different conceptual levels of the speech understanding process. Thus, levels in Hearsay-II contained hypotheses regarding parameters of acoustic signalls, segments of such signals, syllables, words, word sequences, phrases, and interpretations, respectively (see Figure 2.1). This approach has been adopted in other blackboard systems since. Examples are HASP/SIAP (Nii et al., 1988), Crysalis (Engelmore \& Terry, 1979; Terry, 1988), and OPM (Hayes-Roth \& Hayes-Roth, 1979).

Some blackboard frameworks, such as Hearsay-III (Balzer et al., 1980), POLIGON (Rice, 1986), and GBB (Corkill et al, 1986), extended the subdivision of the blackboard into levels by allowing the data on the blackboard to be structured via class hierarchies common in objectoriented modelling.

In the blackboard system Crysalis, sets of blackboard levels were grouped together for the first times to form distinct level hierarclies, called panels. Crysalis, also known as SU/P (Engelmore \& Terry, 1979; Jerry, 1988), is a blackboard system for inferring the atomic structure of proteins from X-ray diffraction data. One panel was used for storage of observable data, while another panel was used for storage of hypotheses derived from this datia and other hypotheses. The opportunistic planning model OPM used a smilar technique. Here, the sets of levels were called planes. The blackboard in OPM contained five planes coinciding with different kinds of decisions in its domain of erand planning 


\begin{tabular}{|c|c|c|c|}
\hline & $\begin{array}{l}\text { executive } \\
\text { priorities } \\
\text { focus } \\
\text { schedule }\end{array}$ & $\begin{array}{l}\text { meta plan } \\
\text { problem definitio } \\
\text { model } \\
\text { policies } \\
\text { evaluation criteri }\end{array}$ & \\
\hline \multicolumn{2}{|c|}{ plan abstraction } & knowlegde base & plan \\
\hline \multicolumn{2}{|l|}{$\begin{array}{l}\text { intentions } \\
\text { schemes } \\
\text { strategies } \\
\text { tactics }\end{array}$} & $\begin{array}{l}\text { errands } \\
\text { layout } \\
\text { neighbors } \\
\text { routes }\end{array}$ & $\begin{array}{l}\text { outcomes } \\
\text { designs } \\
\text { procedures } \\
\text { operations }\end{array}$ \\
\hline
\end{tabular}

Figure 2.2: Blackboard panels in OPM.

(see Figure 2.2). These five planes are called executive, meta-plan, plan abstraction, knowledge base, and plan. See (Hayes-Roth \& Hayes-Roth, 1979) for a description of the semantics of the planes and levels in OPM.

A part from subdividing the blackboard, structuring can be achieved also by representing relations between the data elements on the blackboard to model dependencies and inference relations or to indicate, e.g., partwhole relationships. Binary relations are fairly common in blackboard architectures and are indicated usually by the term links.

\subsubsection{Communication medium}

In this section, we discuss the blackboard in its role as a communication medium. An important function of a blackboard in a blackboard system is to provide a unfied communication medium for exchanging data between otherwise independent programs (the knowledge sources) within a complete blackboard system. Knowledge sources in a blackboard architecture are independent in the sense that they are capable of independently executing a certain subtask, solve a given subproblem, generate an hypothesis, or perform some other step in the problem-solving task of the complete blackboard system. Knowledge sources are not completely independent, as they have to be combined in some (possibly very complex) way to solve a given problem cooperatively. Communication is instrumental in achieving cooperation. 


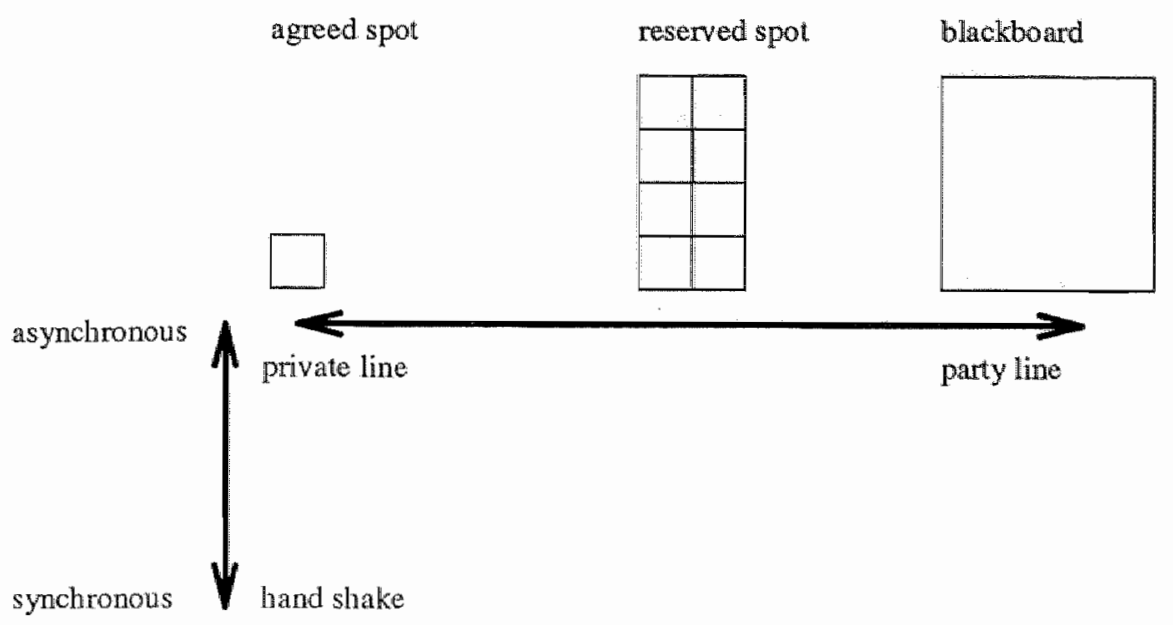

Figure 2.3: Options in communication.

In (Winston, 1984, Chapter 5) an abstract description of options for communication between different computer programs or agents is presented. These options are shown in Figure 2.3. Options can be subdivided into two different classes: direct communication (or privateline method) and indirect communication (or party-line method). The private-line method is only applicable when it is known exactly which programs will engage in a certain communication. In that case information can be sent directly to the intended receiving program. The privateline methor can be implemented in a synchronous as well as in an asynchronous fashion. Synchronous implementation implies that both sender and receiver need to be engaged in the information exchange simultaneously at some point in time (also metaphorically termed a handshake). In asynchronous form a message is stored by the sending program at an agreed spot and inspected by the intended receiver at an appropriate, later, time, while the sending program may proceed undisturbed after sending.

In order to increase independence of knowledge sources in blackboard architectures, it is often not desirable (or, indeed, not even possible) that knowledge sources know which other components in the system (knowledge sources or control unit) will use exported clata. Thus, the 
private-line method is not a good candidate for communication between the knowledge sources.

In the party-line method, the asynchronous mode of private-line communication is generallised. Messages are stored again at an agreed spot, but now more than one other program can inspect the contents. Here, any variation along a spectrum is possible: at the far end is the blackboard method (so termed by Winston). In the blackboard method all messages are stored in one big, general space (the blackboard), where all programs can inspect all messages. The other end of the spectrum is represented by the private-line method. Between the two extremes in this spectrum is the reserved-spot method, which allows only selected programs to write or inspect certain message storage methods. These separate message storage methods play different roles: they contain either different "kinds" of information or they are used to set up communication between different (groups of) programs.

Hearsay-II (like most other existing blackboard systems) uses a form of the reserved-spot method for data exchange between knowledge sources. ${ }^{1}$ In Hearsay-II the blackboard is subdivided into levels and knowledge sources typically only inspect data on one level and write data on another level. The different levels of the blackboard in Hearsay-II coincide with different interpretational levels in the speech understanding domain, for which Hearsay-II was developed, such as syllables, words, and phrases.

An advantage of the asynchronous communication method between different programs lies in the fact that it allows communication to take place between programs which are not running at the same time: data can be stored on the blackboard for future reference by then existing programs. Many blackboard architecture implementations need such a mechanism when no more than one knowledge source can be executed at the same time. Thus, messages must be saved at least until the appropriate knowledge sources have retrieved the information they need.

An example of using a blackboard purely as asynchronous communication medium can be found in (Tsichritzis et al., 1987), which describes an object-oriented enviromment with a blackboard for communication among its objects.

\footnotetext{
${ }^{1}$ Hearsay-II also allowed some direct communication among knowledge sources, i.e., communication using the private-line method.
} 


\subsection{Knowledge sources}

The knowledge sources in a blackboard system can be seen as conditionaction pairs: the condition part of a knowledge source specifies interesting situations for that knowledge source, i.e., the data that needs to be present on the blackboard before the action part associated with that knowledge source can be executed. In this section we focus in turn on issues related to condition parts and action parts. Furthermore, we discuss consequences of the strict modularity of a blackboard system obtained by subdividing the system into knowledge sources.

\subsubsection{Condition part}

The condition part of a knowledge source specifies interesting situations for contribution to the problem-solving process by the corresponding knowledge source in terms of data that must be present on the blackboard; it is the component of a knowledge source used as a template for scanning the blackboard for opportunities to contribute to the overall problem-solving process. In many blackboard-architecture implementations this scanning is facilitated by the use of events. Events are representations of changes of information on the blackboard rather than of the information itself. Not only do events provide in some cases a more natural way of representing interesting situations on the blackboard, but often can they be used more efficiently as well. Events make it possible for knowledge sources to be considered only when a. specified change in the blackboard situation occurs, instead of regularly checking large sections of the blackboard for the presence of interesting data. The part of a condition part specifying in which events a specific linowledge source is interested is called usually a trigger. Starting with the Hearsay-II system, virtually all blackboard architectures have employed an eventbased mechanism for determining applicability of linowledge sources.

Events are not always sufficient for representing a condition; additional blackboard state-based condition checking may be mandatory to ensure that a knowledge source is indeed applicable. Thus, a typical condition part consists of two parts: one specifying interesting blackboard state changes and one specifying additionally necessary information. Important blackboard architectures like Hearsay-II and BB1 (Hayes-Roth, 1985) use this subdivision. On the other hand, HASP/SIAP (Nii et al, 
1988) and the blackboard framework AGE (Nii \& Aiello, 1979), which is related to HASP/SIAP, rely completely on events for deciding on the applicability of knowledge sources.

\subsubsection{Action part}

The action part of a knowledge source contains the code which actually can contribute to the problem-solving process. Based on the information that caught its attention in the first place (the data on the blackboard responsible for the condition to be satisfied), the action part proceeds and eventually modifies the blackboard according to its findings. The action part can read and use additional information from the blackboard for this task and later create new information on the blackboard or modify or delete existing data on the blackboard, thus possibly making new opportunities for other knowledge sources to contribute.

Some blackboard architectures use IF-THEN rules for implementing the action parts of their knowledge sources, such as AGE (Nii \& Aiello, 1979) and BB1 (Hayes-Roth, 1985). Other systems use arbitrary code, such as SAII code in Hearsay-II (Erman et al, 1980), LISP code in HearsayIII (Balzer et al., 1980), or Pop-11 code in Blondie (see next chapter). One of the interesting features of the blackboard architecture in general is the possibility to use different representational techniques for the action parts of different knowledge sources: the blackboard architecture can be used for incorporation of subsystems into one system, each using different knowledge representation and inferencing techniques. The section on blackboard applications later in this chapter gives examples of blackboard applications exploiting this feature.

\subsubsection{Knowledge-source instantiations}

When a knowledge source becomes applicable to certain information on the blackboard, the knowledge sonrce and this information together form an instantiation for that knowledge source. Especially in blackboardsystem implementations, where only one knowledge source can be executed at the same time, there might elapse some time between the time a knowledge source becomes applicable and the time it is executed. Blackboard systems such as Hearsay-II (Erman et al, 1980) and BBI (Hayes- 
Roth, 1985) represent knowledge-source instantiations via specific data structures. In BB1 these data structures are called knowledge-source activation records or KSARs. These terms have since been used in other publications as well. Representations of knowledge-source instantiations are necessary to distinguish between different instantiations which may exist at the same time for the same knowledge source and to store information about each specific instantiation for selection purposes. This last aspect enables a control component to use information both about specific information that satisfied the condition of a knowledge source and information about the knowledge source itself for its selection task.

\subsubsection{Modularity}

The blackboard architecture presupposes a subdivision of the problemsolving capabilities of a blackboard system into different modules, i.e., the knowledge sources. Thus, the blackboard architecture is a modular architecture. Modularity is important both from a top-down and from a bottom-up point of view. From a top-down point of view it is interesting to study which criteria. allow for a meaningful partitioning of a given problem-solving task, while on the other hand many of the same aspects may present ideas on how distinct tasks or chunks of expertise can be combined to form a complex problem-solving system.

Partitioning a problem-solving process into separate knowledge sources is a sound practice as proposed by software engineering: solve the problem via a divide and conquer strategy. Combining knowledge sources to form a complete system provides opportunities for integration of heterogeneous components (see Section 2.6 for illustrations).

Modularity of the backboard architecture has further advantages with

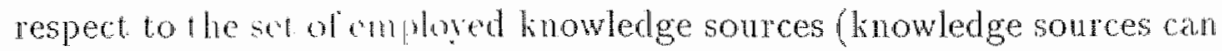
be added, ronowed. or wanged fairly easily) and to concurrency, as is discussed in Sextion : 2.4 .

Somewhat morc abstricly. knowledge sources are actions or objects which can be rasomed ahout. In some blackboard frameworks this issue has received extensive attention. In BB1, for instance, the selection of knowledge sonices for excution is presented as a planning task (Hayes. Roth. 1985). Other approaches rate the expected effects of execution of a knowledge sonrce against the current goals of the system in order 
to display goal-directed behaviour (Corkill et al., 1982; Lesser et al., $1989 b)$.

An interesting question is raised when an actual subdivision into knowledge sources must be developed. One can employ different criteria for deciding what should constitute a knowledge source. In Hearsay-II different knowledge sources coincided mainly with different bodies of expertise (Erman et al., 1980). Other approaches might choose to view knowledge sources as tasks or (sub)processes or as collections of activities having something in common such as the same events needed for triggering or the same data that should be present on the blackboard in order to satisfy a condition. The resulting subdivision may have implications for control (see also the next section). For instance, a stress on events in the definition of knowledge sources will make the use of events for control more natural (event-driven control). On the other hand, a stress on data that must be present will imply a more data-driven form of control.

\subsection{Control}

Blackboard control deals with tasks such as administrating which knowledge sources can be executed and/or which blackboard data need to be processed, selection of appropriate actions, and supervising the execution of knowledge sources. Blackboard control is not essential within the blackboard model of problen solving as described by the blackboard metaphor. In the blackboard metaphor specialists (knowledge sources) are capable of monitoring the blackboard, deciding themselves when and how to contribute to the overall problem-solving process. This presupposes an architecture in which each knowledge source has a processor fully at its own disposal, performing a cycle of alternately checking the blackboard for interesting situations and, if appropriate, executing the corresponding knowledge source. In fact, however, none of the earlier blackboard architectures were developed in a multi-processor environment (the earliest attempt of a concurrent blackboard architecture implementation was described in (Fennell \& Lesser, 1977)). Lack of resources prompted the need for a component within the architecture, the control wnit, that could serve as a scheduler for mediating between knowledge sources competing for limited resources. 


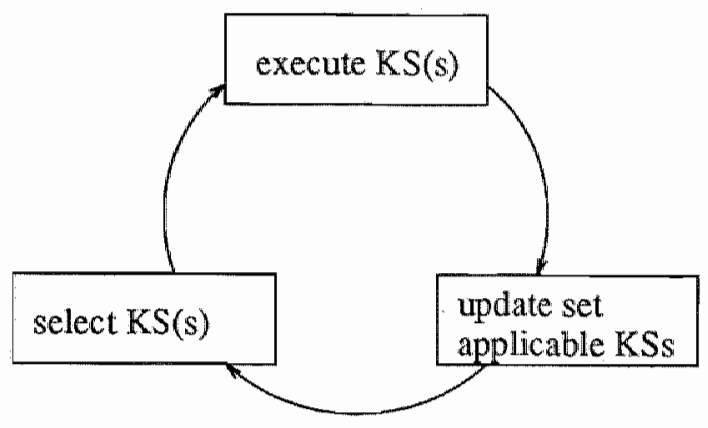

Figure 2.4: Example of a control loop.

The primary objective of a control unit is thus scheduling. Initial blackboard system applications such as Hearsay-II and HASP/SLAP use reasonably simple strategies for selecting knowledge source activities. Later, research efforts have concentrated on extending the control unit, equipping it with the ability to make well-informed, intelligent decisions as to the next step to be taken in the problem-solving process. The blackboard architecture can provide much useful information for the decisionmaking task in the form of problem-solving state information, as represented on the blackboard, and information about activities, as represented in descriptors of the knowledge sources.

Most sequential blackboard systems have a control unit executing a control loop. Typically, such a control loop in turn executes one or several knowledge sources, checks which knowledge sources have become applicable due to recent modifications to the blackboard, and selects one or several of all applicable knowledge sources to be executed next (see Figure 2.4). This section discusses issues important to control in blackboard architectures.

\subsubsection{Terminology}

Top-down and bottom-up processing

The blackboard levels and blackboard panels in many blackboard systems are ordered following a hierarchy. In Hearsay-II some blackboard 
levels coincided with abstraction levels within the domain of speech understanding (including, e.g., levels representing syllables, words, or phrases), while other levels represented an aggregation of information on 'lower' levels (e.g., the level for word sequences aggregating data from the "word" level). When there is such a hierarchy of blackboard levels and panels, we can define the terms top-down processing and bottom-up processing. Top-down processing starts at the highest level according to the hierarchy and uses information on that level to derive intermediate results or hypotheses on lower levels. These intermediate results can then in turn be refined to derive intermediate results on even lower levels of the hierarchy. Top-down processing thus implements successive refinement of intermediate results. Conversely, bottom-up processing starts at the lowest level of the hierarchy and combines intermediate results on lower levels to find intermediate results on higher levels. Bottom-up processing uses successive combining of intermediate results to reach $a$. solution at the top level.

\section{Opportunistic problem solving}

Problem solving is called opportunistic when at each point in the process decisions are made regarding opportunities for proceeding in a specific direction as indicated by the available activities and based on information on past and present states of the process. Opportunistic problem solving might proceed in a top-down or bottom-up fashion, when characteristics of the problem domain suggest such an approach, but is not: restricted to one of these two orderly strategies. Top-down and bottomup processing, e.g., may be intermingled. Also, it might happen that work on one partial solution is abandoned suddenly in favour of another, more promising, partial solution. In opportunistic problem solving, interesting intermediate results are identified and promising activities are chosen to process these interesting intermediate results; 'up', 'down', or in any other appropriate direction.

Some researchers have stated that the blackboard architecture is synonymous with opportunistic problem solving - see, e.g., (Nii, 1986b). However, we tend to agree with Craig that opportunistic problem solving is made possible by the fact that control information and memory can be recorded and made available explicitly, which is not the prerogative of the blackboard architecture. Furthermore, a blackboard system 
can be implemented in such a way that control proceeds completely systematic, as in top-down or bottom-up processing. Still, the blackboard supports opportunistic problem solving, which makes the architecture an interesting candidate when such a form of control is desired.

\section{Data-driven, event-driven, and goal-directed control}

Action selection is said to be data-driven, when selection of activities is based only on currently available input and derived data. (We note that only activities can be selected for execution which are applicable with respect to currently available data.) In purely data-driven control applicable activities are not rated against some goal of the problemsolving process. Event-driven processing emerges, when data changes instead of data itself is used for selection of activities. On the other hand, goal-directed control selects activities promising a contribution to some explicitly stated goal or subgoal.

Control in blackboard architectures is usually data driven and/or event driven. Data about the current state of the problem-solving process is readily available on the blackboard and most, if not all, blackboard architectures use events to decide whether a knowledge source can or: should contribute to the problem-solving process.

'Data-driven control', and 'goal-directed control' are not defined in terms of (abstraction) hierarchies like top-down and bottom-up processing. Although data-driven control often coincides with bottom-up processing and, similarly, goal-directed control might coincide with top-elown processing, these notions can not, and should not, be interchanged. In goal-directed control the effects of an action per se are under consideration and rated against goals and subgoals; the direction of the action according to some hierarchical representation of the problem domain is not an issue.

While opportunistic control can model top-down and botton-up behaviour and (this is the essence of opportunistic control) switch between these as appropriate, opportunistic control does not provide a means for integrating data-driven and goal-directed control in the same way. Integrating data-driven and goal-directed control involves representing current state data and goals simultaneously and using both the relation. ships between problem-solving states and knowlerige-source conditions 
and the relationships between goals and expected knowledge-source results to determine the most appropriate activity at any time. In a sense integrated datamdriven and goal-directed control can be opportunistic again as the best activities are selected according to presently available information.

\subsubsection{Control as a planning problem}

In many cases sophisticated control in blackboard systems has taken the form of a planning problem. Planning can be defined as the activity of generating a sequence of activities which, when executed, achieves one or more goals. Goals are either stated explicitly (e.g., 'put block A on block B') or defined much more loosely: the goal in the - blackboard-based - errand-planning system OPM (Hayes-Roth \& Hayes-Roth, 1979) is to 'generate a realistic plan indicating which errands to do, when, and how to travel among them'.

The similarity between planning and blackboard control is illustrated by the following. In planning domains the input is a current world state, a set of activities, and a set of goals, specifying more or less strict constraints of the desired world state (see, e.g., Vere, 1987). The output is a sequence of activities vltimately satisfying the stated goals. The control process in a blackboard architecture receives as its input the current blackboard state and a set of knowledge sources. The output, as seen in the preliminaries to this section, is a sequence of system activities solving the current problem.

Seemingly, two differences exist between the generic task of planning and the control process in blackboard systems:

- planning tries to find a sequence of actions, while blackboard control (which is predominantly data driven and/or event driven) seems more concerned with selecting the 'best.' next activity (singular);

- a set of goals, although possibly fuzzily stated, is essential in the definition of a planning problem, but does not necessarily appear in the definition of blackboard control. 
The stated differences ${ }^{2}$, however, need not be of relevance. We first show that blackboard control also finds sequences of activities. The simplest strategy for selecting activities in a blackboard system is to execute all knowledge sources in all applicable situations. However, this approach can lead to a combinatorial explosion and is clearly not an efficient, nor an effective one. In most problem domains, available resources like time and processing power limit the number of activities that can be executed. Moreover, the order in which activities are performed may determine whether a solution is found at all. It is the task of the control unit to select and execute activities that solve the current problem, while ensuring that resource constraints are met. When this process is completed successfully, a sequence of activities is found that solved the problem. At the same time this sequence of activities has also been executed, extending the generic task of planning somewhat. The control unit repeatedly selects the next activity or activities, executes them, and uses the resulting problem-solving state information to decide how to proceed next, possibly readjusting previously made decisions, as activities do not always have the desired effect. This adds execution and monitoring to the task of the control unit, but also encompasses planning itself.

In order to show that goals are as essential in blackboard control as they are in planning, let us next consider the relevance of goals in the definition of 'problem' in a blackboard application. Many blackboard systems have been applied to domains, where explicit, a priori formulation of goals is not possible. Examples are the tasks of speech understanding (Hearsay-II, Erman et al., 1980), signal interpretation (HASP/SIAP, Nii et al, 1988), errand planning (OPM, Hayes-Roth, 1985), and protein structure modelling (PROTEAN, Johnson \& HayesRoth, 1986). In these domains (sub)goals can be generated either during the problem-solving process (in PROTCAN) or are implemented implicitly and employed by way of automatic sequencing of activities by means of knowledge-source action-part interactions with the blackboard and matching knowledge-sources conditions (in Hearsay-II). Implicit or explicit, each system has as its most basic goal that of solving problems

\footnotetext{
${ }^{2}$ One might also argue that there exists a difference in granularity between the actions in a plaming problem and the knowedge-sonce actividias in blackboard control. This implies that planing systems and blackboard control components operate on different levels of abstraction.
} 
in its application domain. No blackboard system is capable of intelligent behaviour unless it uses a goal-oriented process as part of control. Thus, control in blackboard systems, in a manner similar to planning, receives goals, although possibly implicitly stated, as its input.

Thus, we have illustrated that control in blackboard architectures does not really differ from planning problems. Therefore, blackboard control can benefit from ideas and insights acquired about the generic task of planning, including such concepts as hierarchical planning (implemented by ordering goals and subgoals), temporal planning (e.g., in applications in process control), and distributed planning (for distributed blackboard architectures). An important example of viewing blackboard control as a planning process can be found in (Hayes Roth \& HayesRoth, 1979; Hayes-Roth, 1985; see also Section 2.5.2). The planning view was adopted also in the work by Durfee on partial global plans (Durfee, 1988), where partial plans are used by cooperating, "intelligent" systems (complete blackboard systems) to reason about mutual intents and purposes.

In some respects the planning view of blackboard control seems to be violating the inherent data-driven and event-driven character of the blackboard architecture. Techniques resulting from research into the area of situated action (see, e.g., Agre, 1990) may be useful for implementing sophisticated control mechanisms for blackboard systems which are more natural to the blackboard architecture in that these techniques would be able to select activities based on the 'sea of data' available to the control component of a blackboard system at any point in time.

\subsection{Concurrent blackboard architectures}

The blackboard architecture has several characteristics maling it suited for concurrent implementations. In fact, many such implementations exist. This section discusses concurent blackboard architectures. A distinction is made between parallel blackboard architectures and distributed blackboard architectures. Parallel blackboarcl architectures are characterised by concurrently executed knowledge sources communicating via a single, shared memory blackboard. In distributed blackboard architectures not only can knowledge sources be concurrently executed, but the blackboard is distributed as well. 


\begin{tabular}{|c|c|c|}
\hline reference & mame & application \\
\hline (Märtin \& Waldhör, 1988) & Basar & office automation \\
\hline (Adey, 1988) & - & ship and aircraft control \\
\hline (Aiello, 1986) & $\mathrm{CAGE}$ & \\
\hline (Shafer \& Stentz, 1987) & CODGER & robot navigation \\
\hline (Fehling et al, 1989) & HCVM & $\begin{array}{l}\text { real-time control of } \\
\text { materials composition } \\
\text { process }\end{array}$ \\
\hline (Raulefs, 1987) & HCVM-2 & - \\
\hline (Fleisch, 1990) & Mirage & - \\
\hline (Pomeroy \& Irwing, 1990) & $\begin{array}{l}\text { Pilot's } \\
\text { Associate }\end{array}$ & pilot assistance \\
\hline (Velthuijsen et al., 1987b) & Blondie-II & - \\
\hline (Fennel] \& Lesser, 1977) & Hearsay-II & speech understanding \\
\hline (Talukdar et al., 1988) & DPSK & engineering wasks \\
\hline (Winkelbauer, 1988) & KIM & $\begin{array}{l}\text { integrating coupled } \\
\text { systems }\end{array}$ \\
\hline (Ling et al., 1987) & - & - \\
\hline (Ensor \& Gabbe, 1985) & - & $\begin{array}{l}\text { transactional } \\
\text { blackboards }\end{array}$ \\
\hline
\end{tabular}

Table 2.1: Parallel blackboard architectures.

\subsubsection{Parallel blackboard architectures}

As we have seen earlier, the blackboard provides a logical mechanism for asynchronous communication between different programs. In a blackboard architecture, knowledge sources interact only by means of the blackboard. Knowledge sources 'Jook' at the blackboard to see if suitable data is present to trigger their execution. If they have been selected for execution, the result of their execution will be new or altered data on the blackboard, again triggering (other) knowledge sources. Solving a problem using a blackboard architecture is based on the cooperation of the knowledge sources present.

But this cooperation need not rely on the actual location of the knowledge sources: knowledge sources can very well be distributed over different processors and still contribute to the overall problem-solving prow cess. Such a blackboard architecture, where knowledge sources can be executed concurrently, but communicate via a single shared memory 
blackboard, is called a parallel blackboard architecture. Numerous examples of parallel blackboard architectures can be found in the literature (see also Table 2.1). Basar (Märtin \& Waldhör, 1988), CAGE (Aiello, 1986), CODGER (Shafer \& Stentz, 1987), the Heuristic Control Virtual Machine (HCVM, Fehling et al., 1989), HCVM-2 (Raulefs, 1987), Mirage (Fleisch, 1990), and Pilot's Associate (Pomeroy \& Irving, 1990) are all parallel blackboard systems or frameworks. Another example of a parallel blackboard architecture is Blondie-II, which is further described in Chapter 4 of this thesis. In (Fennell \& Lesser, 1977) an early study into the possibilities of parallelising the blackboard architecture is described. In that study, efficiency of a parallel implementation of a subset of the Hearsay-II system was compared with a sequential implementation of the same subset. In these experiments speed up factors of one order of magnitude were achieved.

Evidently, speed up is often a major goal of parallelisation efforts. Although the blackboard architecture has some inherently concurrent characteristics, many application domains prohibit extensive, many orders of magnitude, speed up factors. Rice suggests that, until really large knowledge bases are used and longer inference chains are needed, the most promising results should be expected from data parallelism, as opposed to pipeline parallelism ${ }^{3}$ (Rice, 1989c).

Another major goal for developing a parallel blackboard architecture is integration of very diverse processes. An example is the integration of symbolic and numerical processes. The Distributed Problem Solving Kernel (DPSK, Talukdar et al., 1988) and the Knowledge-Based Integration Manager (KIM, Winkelbauer, 1988) are examples of parallel blackboard frameworks designed with exactly this purpose in mind. A further step is presented in (Ling et al., 1987) describing the design of a. VLSI architecture including both numerical and symbolic processing units.

\footnotetext{
"In general, two distinctive opportunities for concurrency exist in data processing. Usually, the same sequence of processing activities needs to be applied to a number of data elements. Such a sequence is called a pipeline metaphorically. One approach to introducing concurrency is by execnting the activities in a pipeline in parallel, while the data elements are passed from one processing element, executing one activity in the pipeline, to the next. This approach is called pipeline parallelism. In the other approach, called data parallelism, the data elements are processed in parallel, i.e., by executing the pipelines concurrently.
} 
Theoretical research in the area of description languages for distributed cooperative systems provides another incentive for studying parallel blackboard architectures than speed up and integration requirements alone. Kelemen describes results of research studying formal grammars and languages for modelling distributed and cooperative systems (1991). The proposed grammars use a blackboard-like approach for interaction between otherwise independent 'ordinary' grammar systems. One of these results states that the generative power of such cooperating grammars is stronger than the power of the constituting grammars alone. This result suggests that parallel blackboard systems can be used to implement applications consisting of cooperating subsystems (knowledge sources) using relatively simple formalisms for reasoning which are more powerful than would be possible by implementing the complete system as a sequential system based on the same simple formalisms.

The most significant complications in parallel blackboard system implementations occur with concurrent access to the (single) blackboard:

- Concurrent access to the blackboard needs to be sequentialised for the process containing the blackboard to be able to handle simultaneously arriving requests.

- Consistency of data on the blackboard can be violated by concurrent access. When a knowledge source wishes to modify the blackboard based on information obtained earlier, this information may have been changed meanwhile by other knowledge sources, thus leading to inconsistencies. Several parallel blackboard ar. chitectures use locking (prolibiting access of blackboard objects presently being modified by a knowledge source) or tagging (annotating blackboard objects to maintain a history of updates) mechanisms in order to prevent such inconsistencies to occur (see also Chapter 4). Neither of these options is really satisfactory. Locking will lead to delays in knowledge sources as they wait for access of locked regions of the blackboard. Tagging, on the other hand, does not block parts of the blackboard from use by other knowledge sources, but leaves room for a knowledge source to proceed only to find that the information on which processing is based has become obsolete in the mean time. A transactional framework for ensuring consistency of data on the blackboard was proposed in (Ensor \& Gabbe, 1985). 
Finally, we mention POLIGON (Nii, 1986c; Rice, 1986; Rice, 1989b; Rice \& Aiello, 1989). POLIGON supports not only concurrent execution of activities, but also a distributed blackboard. In this respect, POLIGON is both a parallel and a distributed blackboard architecture. POLIGON is more extensively described in Appendix A.

\subsubsection{Distributed blackboard architectures}

While parallel blackboard architectures employ a shared memory approach with respect to the blackboard, distributed blackboard architectures are characterised by a distributed memory blackboard. Quite naturally, a distributed blackboard leads to distributed control: because the blackboard is distributed, the occurrence of interesting situations on the blackboard, as well as events, is distributed as well. Thus, determination of applicability of knowledge sources may occur locally (per blackboard). The virtual blackboard model, as proposed in (Jagamnathan, 1989), treats the (possibly actually distributed) blackboard as only one blackboard, but recognises different control components, each controlling a separate set of knowledge sources which together are interested primarily in only one section of the complete virtual blackboard. Distributed blackboard systems, each with its own blackboard, set of knowledge sources, and local control component, typically consist of a network of several 'ordinary" blackboard systems cooperatively worlking on a common problem.

Distributed Artificial Intelligence (Davis, 1980; Davis, 1982; Fehling \& Erman, 1983; Smith, 1985; Gasser, 1987; Sridharan, 1987; Huhns, 1987; Gasser \& Huhns, 1989; Bond \& Gasser, 1988) is the research area concerned with the study of more or less independent systems cooperatively solving a shared problem by making informed decisions regarding com.munication among themselves. Decisions involve when and with which other systems specific data should be shared and how (sub)tasks can be exchanged effectively. Networks of more or less independent, 'intelligent' subsystems, which are the subject of distributed AI research, are often termed distributed problem-solving networks, where the subsystems are the nodes in the network.

Distributed blackboard architectures have been used extensively as an underlying architecture for research in distributed AI as they can provide the more or less independent systems that malie informed decisions 


\begin{tabular}{|l|l|l|}
\hline reference & name & application \\
\hline (Lesser \& Corkill, 1983) & DVMT & vehicle monitoring \\
(Brajnik et al., 1988) & ADL & - \\
(Buteau, 1989) & CAIBL & - \\
(Craig, 1989) & CASSANDRA & - \\
(Ayel, 1988) & CSM & computer aided \\
(Leao \& Talukdar, 1988) & COPS & manufacturing \\
(Saxena et al., 1990) & DISPROS & air traffic control \\
(Weihmayer \& Brandau, 1990) & TEAM-CPS & network control \\
(Jagannathan, 1989) & Erasmus & - \\
(Addison \& Leon, 1987) & - & information flow \\
(Ciscon \& Johnson, 1990) & - & control \\
(Harmon, 1989) & & robot path \\
(Liempd et al., 1989) & planning \\
(Dai et al., 1990) & Blondie-III & robot control \\
(Ling et al., 1987) & HOPLS & realutime \\
\hline
\end{tabular}

Table 2.2: Distributed blackboard architectures.

about their activities, including communication. Cooperation between the nodes in a network of blackboard systems is achieved when a knowledge source at one node can investigate the blackboard of another node and/or put data there, directly or indirectly. Knowledge sources of different nodes can interact in a way similar to the way linowledge can sources within a single blackboard system. This way a blackboard can be used to exchange not only information, but also fasks between the nodes in a distributed blackboard system: the appearance of new or modified data from one node on the blackboard at another node may lead to the creation of a corresponding event, in turn causing triggering and execution of one or more knowledge sources in that other node. The actual selection for execution of a linowledge source triggered in this manner remains the responsibility of the control wnit of the receiving node. Thus, execution is not a 'sure' consequence of data exclange.

Examples of distributed blackboard systems and frameworks are the following (an overview is given in Table 2.2): the Distributed Vehicle Mon- 
itoring Testbed (DVMT, Lesser \& Corkill, 1983), the blackboard specification language ADL (Brajnik et al., 1988), CAIBL (Buteau, 1989), CASSANDRA (Craig, 1989), the Conceptual Supervision Model (CSM, Ayel, 1988), COPS (Leao \& Talukdar, 1988), DISPROS (Saxena et al., 1990), and the research testbed TEAM-CPS (Weihmayer \& Brandau, 1990). The blackboard generator system Erasmus (Jagannathan, 1989) allows also the specification of distributed blackboard systems. Additional examples are given in (Addison \& Leon, 1987; Ciscon \& Johnson, 1990; Harmon, 1989). Chapter 5 contains a description of the distributed blackboard framework Blondie-III and its application to a telecommunications network configuration problem.

A network of parallel blackboard architectures forms a hybrid version of a parallel and a distributed blackboard architecture. HOPES (Dai et al., 1990) is an example of such a hybrid architecture with a distributed hierarchy of blackboard panels and concurrent agents gathered around each blackboard panel. In (Ling et al., 1987) a VLSI design for a hybrid blackboard architecture is presented. This architecture also integrates symbolic and numerical processing units within its structure. An overview of the different opportunities for exploiting concurrency in the blackboard architecture is given in (Corkill, 1988),

\subsection{Cognitive models}

Although important aspects of the blackboard architecture can be explained and illustrated by means of its metaphor rather easily, experience with blackboard applications has taught us that developing blackboard systems may be far from simple. Common problems in developing blackboard systems are: determining how the system should be subdivided into knowledge sources; deciding the way the blackboard should be structured; determining how events should be used to ensure that the problem-solving process continues until a solution is found; and, finding an efficient control scheme. In view of these problems it might be suggested that the blackboard architecture is an 'unnatural' comptr. tational frameworli. To illustrate that this is not true in general, this section describes cognitive models for problem solving in two different task domains (reading and planning), both based on the blackboard architecture. One of these, a model for planning, has actually been im- 
plemented as a blackboard system. The other, a model for reading, does not describe a blackboard architecture in the strictest sense as it does not include knowledge sources and a blackboard in the usual way.

\subsubsection{A blackboard model of reading}

McClelland and Rumelhart have developed a cognitive model of reading that was inspired on ideas apparent in early publications on Hearsay-II (McClelland \& Rumelhart, 1981; Rumelhart \& McClelland, 1982). We discuss these ideas first and then proceed by presenting this cognitive model.

As can be seen from early publications on the evolution of the Hearsay-II architecture from its predecessor Hearsay-I (Reddy et al., 1973), the original architecture of Hearsay-II was intended to provide a system in which knowledge sources would operate as paralle], independent processes, cooperating to interpret speech utterances by using a hypothesize-and-test paradigm. In the hypothesize-and-test paradigm knowledge sources create hypotheses in a. shared memory which can be reviewed later by other knowledge sources that test and possibly alter or remove these hypotheses. This way uncertainties and errors, which can not be resolved by any knowledge source alone, are resolved as more information becomes available through continued processing by other knowledge sources. Actual parallelism was not implemented until much later (Fennell \& Lesser, 1977 ), but it was an important aspect of the original view of the architecture nonetheless. A discussion of possible ways for exploiting parallelism and expected consequences was included in (Lesser et al., 1975).

Also important in the original ideas on the architecture of Hearsay-II and the hypothesize-and-test paradigin were the different levels of abstraction and/or aggregation in the representation of the problem domain. This led to a hierarchy of levels for the representation of hypotheses in the shared memory. Knowledge sources process information on one level of representation and often can acd or modify hypotheses on another level of representation accordingly. Knowledge sources may process in both directions in the representation hierarchy. Essential to the hypothesize-and-test paradigm is that when processing on one level of representation halts as a result of insufficient data, it can benefit from ongoing processing on other levels. Thus, processing on different levels 
of representation can drive each other to an overall solution even in cases where information on each level alone would be insufficient.

The hypothesize-and-test paradigm is useful in resolving uncertainty. In a task like speech understanding each level of representation involves many causes for uncertainty starting with noise at the acoustic level and further affected by such phenomena as pauses, unnecessary phrase repetitions, and indistinguishable alternative word sequences (Erman et al, 1980). As has been shown with the development of HearsayII, information on one level can be used to resolve the uncertainty on another level.

The original model of parallel sources of knowledge, cooperating in a hypothesize-and-test fashion as described above, inspired McClelland and Rumelhart to clevelop a model of reading, called interactive activation model (McClelland \& Rumelhart, 1981, Rumelhart \& McClelland, 1982). Moreover, many of the ideas in their work on Parallel Distributed Processing in the field of neural networks, were inspired by the hypothesize-and-test characteristics of the blackboard model:

"In 1975, Rumelhart outlined a model of reading called the interactive model. That model, inspired by the Hearsay model of speech understanding (Reddy, Erman, Fennell, \& Neely, 1973), supposed that reading involved simultaneous processing at a large number of levels, including visual feature, letter, word, syntactic, and semantic levels. Hypotheses at each level were activated when active hypotheses on adjacent levels suggested them and competed with alternative hypotheses at the same level. This model, of course, was a precursor of the interactive activation model of word recognition and of the approach that underlies this whole book." (McClelland, 1986, p. 122)

The interactive activation model is based on a number of assumptions.

1. It is assumed that perceptual processing in word reading involves different levels of representation that correspond with visual features, letters, and words and higher levels derived from the interpretation of words based on their place in sentences. 


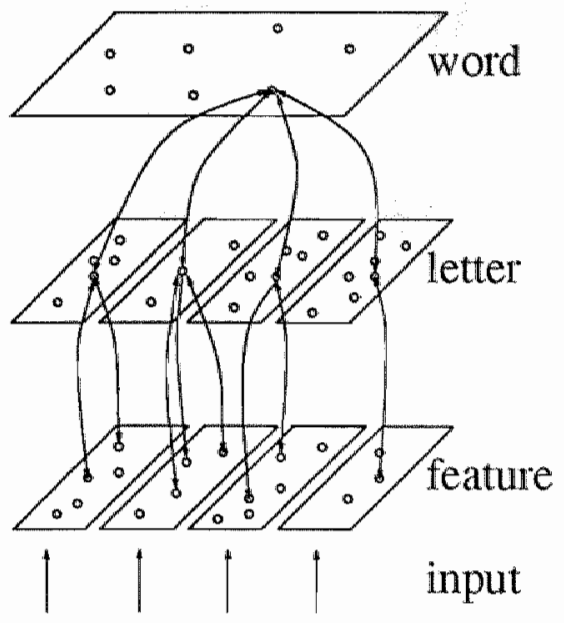

Figure 2.5: Simplified illustration of the interactive activation model.

2. A second assumption states that visual perception involved in reading is essentially parallel. Parallelism here includes spatial parallelism (letters in a word are interpreted - at least in part in parallel) and level parallelism (processing on different representational levels occurs in parallel).

3. McClelland and Rumelhart assume that reading is an interactive process. Top-down and bottom-up processing are integrated and mutually beneficiary.

These assumptions are all covered by one or another aspect in the original development of the Hearsay-II architecture as seen above.

Figure 2.5 illustrates the word recognition model built by McClelland and Rumelhart based on these assumptions. The model consists of three representational. levels: feature level, letter level, and word lenel. The model associates a certain amount of activation (confidence) with each hypothesis on the different levels. Confidence in a hypothesis on one level can increase (or decrease) the level of confidence currently held for hypotheses on other levels, when they are mutually consistent (or inconsistent). These influences are represented by connections between hypotheses on different levels in the figure. This way a connection network 
emerges. Two different elements on the same level of interpretation are either hypotheses for different items or competing hypotheses. Therefore, no stimulating (excitatory) connections exist among hypotheses within one level. We note that the assumption of spatial parallelism implies that hypotheses for different items can coexist. Processing knowledge in this model is represented by the connectivity and by the strengths of excitation or inhibition connections. Connectivity corresponds here to the conditions of knowledge sources in the Hearsay-II architecture, while the strengths of connections can be seen as the action parts of knowledge sources. The hypotheses correspond with the blackboard elements in Hearsay-II.

McClelland and Rumelhart presents an implementation of this model and a number of experiments with the implementation (McClelland \& Rumellart, 1981). Inputs for these experiments were (possibly mutilated) letter features of words and non-words consisting of four letters. The experiments show that the model is capable of accounting for a number of characteristics of human reading skills for these words known from the literature, suggesting that there might be some truth in the assumptions to the model.

Spatial parallelism in the interactive activation model of reading implies that features of different letters and their interpretations may be processed at the same time, suggesting that processing knowledge (as represented by connections between the hypotheses) needs to be duplicated for the different interpretational tasks. The programmable blachboard model of reading (McClelland, 1986) proposes a model in which processing knowledge is represented only once and appropriate parts of the connection network are 'programmed' with this processing lnowledge where needed in order to be ab]e to process different items simultaneously. This approach is not unlike the use of knowledge source instantiations on different data.

\subsubsection{A blackboard model for planning}

Hayes-Roth and Hayes-Roth have noted some remarkable similarities between the way people perform everyday planning tasks and the way the blackboard model of problem-solving operates (Hayes-Roth \& HayesRoth, 1979). Their work was based on a number of protocols describing 
the way in which subjects try to plan a day of doing errands. In the examples there were more errands to run then could be done in the allotted time. The task was to find a plan specifying which errands to run, in what sequence, and how to go from one errand to the next.

Inspection of the protocols revealed the following characteristics. At least two dimensions were relevant in expressing (partial) plans: time and abstraction or level of detail, thus specifying a solution space. During the planning task, the subjects tended to develop plans incrementally, extending partial plans rooted in different points in time and that were formulated in different levels of detail. Moreover, the subjects jumped from one point in the solution space to another; from one partial plan to another. In doing so, the subjects took advantage of opportunities for proposing partial plans, extending partial plans, combining partial plans, or discarding plans that were no longer seen as viable.

Hayes-Roth and Hayes-Roth proposed a model, based on the blackboard, to explain the observed behaviour of subjects. This model assumes that the cognitive planning process can be subdivided into many distinct specialist processes ${ }^{4}$ : some propose detailed plans, while others might suggest modifications of more abstract plans, etc. The suggestions or decisions are recorded on a common data structure, the blackboard. Thus, specialists can inspect and modify plans proposed by other specialists. In the model the blackboard is subdivided into different panels to store different linds of suggestions or decisions and each panel is subdivided into different levels to distinguish between different abstraction levels for the partial plans. A specialist has a condition associated. with it specifying the situations in which the specialist may be able to contribute.

Simulations with an implenentation of this model, called Opportunistic Planning Model (OPM), revealed some characteristics similar to those observed in the protocols of human planning behaviour. These communalities include maling proposals and modifications of partial plans at different levels of abstraction and alternately maling a number of related decisions or jumping about the blackboard.

\footnotetext{
"Specialist proceses in this moded are small, demonulike subprocesses of the coynitive process of phaming dodicated to highly specialised actiwities. Thus, the hem "specialist" in this context should not he confused with the same term in the blackboard metaphor as accorbed in the Chapter 1.
} 


\begin{tabular}{|l|l|}
\hline category & problem addressed \\
\hline interpretation & $\begin{array}{c}\text { inferring situation descriptions from } \\
\text { sensor data } \\
\text { inferring situation descriptions from } \\
\text { sensor data and updating these } \\
\text { descriptions as new data arrives } \\
\text { configuration } \\
\text { constructing an object or system from } \\
\text { elementary building blocks to satisfy } \\
\text { prespecified constraints } \\
\text { control }\end{array}$ \\
configuration of activities to form plans \\
interpreting, predicting, repairing, and \\
monitoring system behaviours
\end{tabular}

Table 2.3: Blackboard-application categories.

'This model of planning diverges significantly from other, 'classical' AI planning methodologies (Wilkins, 1988; Hendler et al., 1990). The opportunistic planning model is much less systematic. In the experiments with human subjects (as well as with an implementation of the model) described in (Hayes-Roth \& Hayes-Roth, 1979) it appeared that the subjects at times completely redirected their activities in order to accommodate newly detected insights. 'Classical' AI planning techniques, on the other hand, usually employ more straightforward strategies, such as successive refinement of goals.

\subsection{Blackboard applications}

In this section we give an overview of existing blackboard applications to illustrate the range of domains in which the blackboard architecture has been used. We have grouped applications together into four application categories: interpretation, monitoring over time, planning and configuration, and control. We use defintions for these categories as shown in Table 2.3 (see also Hayes-Roth et al., 1983, p. 14). The definition of 'monitoring over time' differs slightly from the one given for 'monitoring' in (Hayes-Roth et al., 1983).

Apart from the four sections presenting blackboard applications in each of these four categories, we have included sections discussing applications 


\begin{tabular}{|c|c|c|}
\hline reference & name & application \\
\hline (Erman et al., 1980) & Hearsay-II & speech understanding \\
\hline (Draper et al., 1988) & UMass & image understanding \\
\hline & Schema System & \\
\hline (Nagao et al., 1988) & - & analysis aerial \\
\hline (Andress \& Kak, 1988) & PSEIKI & vision \\
\hline (Williams, 1988) & TRICERO & signal understanding \\
\hline (Srihari et al., 1987) & ABLS & locating address blocks \\
\hline (Dreschler-Fischer, 1986) & - & data fusion \\
\hline (Brogi et al, 1988) & - & data fusion \\
\hline Grumbach \& & $\mathrm{RC}_{2}$ & signal interpretation \\
\hline Ducasse, 1988) & & \\
\hline (Cullingford, 1981) & DSAM & $\begin{array}{l}\text { newspaper article } \\
\text { understanding }\end{array}$ \\
\hline (Aiello, 1983) & PUFE & medical diagnosis \\
\hline (Lenat et al., 1983) & DOLPHIN & $\begin{array}{l}\text { military intelligence } \\
\text { analysis }\end{array}$ \\
\hline (Hausen-Tropper, 1989) & - & weather predictions \\
\hline
\end{tabular}

Table 2.4: Interpretation applications.

exploiting the blackboard architecture as an environment for integrating diverse components and developing complex systems, respectively.

\subsubsection{Interpretation}

The blackboard architecture has been used many times for applications involving interpretation of (large amounts) of data. We present some of these applications in this section. Table 2.4 presents an overview of the applications addressed in this section.

The first implementation of the blackboard architecture, the Hearsay-II speech understanding system (Erman et al., 1980), was also the first example of such an application. Basic advantages of the blackboard architecture for this class of application can be found in the avalability of the blackboard for representation of tentative partial solutions, resolving uncertainty as more and more information has been processed, and the knowledge source modularity which can be used to subdivide processing 
subtasks in a natural way.

Several blackboard systems have been developed for the interpretation of visible signals: the UMass Schema System for image understanding (Draper et al., 1988); a system for structural analysis of complex aerial photographs (Nagao et al., 1988); PSEIKI for computer vision (Andress \& Kak, 1988). TRICERO is a system for signal understanding which consists of a number of different, cooperating blackboard systens (Williams, 1988). ABLS (Address Block Localisation System, Srihari et al., 1987) locates address blocks on mail pieces. Fusion of data from different sources by means of a blackboard architecture has been addressed in (Dreschler-Fischer, 1986; Brogi et al., 1988).

RC2 is a blackboard framework developed for signal interpretation tasks in general and for speech understanding tasks specifically (Grumbach \& Ducasse, 1983).

A collection of other blackboard system applications to interpretation tasks are the following. DSAM (Distributable Script Applier Mechanism, Cullingford, 1981) used a blackboard architecture for its task of understanding newspaper articles on plane crashes. In (Aiello, 1983) three different versions of a system for diagnosis of pulmonary disease (PUFF) are described, each developed with the blackboard framework AGE (Nii \& Aiello, 1979). DOLPHIN was developed for the interpretation of military intelligence reports (Lenat et al., 1983). Additionally, weather prediction has been tackled with a blackboard architecture as described in (Hausen-Tropper, 1989).

\subsubsection{Monitoring over time}

Although the kind of task meant by 'monitoring over time' seems, at least superficially, similar to the kind of tasks addressed in the previous section, there is an important difference. Monitoring over time obviously includes a time aspect as information to be interpreted arrives over a period of time, and interpretation must be based on current information and be updated with newly arriving data. Conversely, ordinary interpretation systems can suffice with interpretation of one batch of data, while discarding earlier found interpretations and start anew for eventual successive inputs. 


\begin{tabular}{|c|c|c|}
\hline reference & name & application \\
\hline (Nii et al, 1988) & HASP/SIAP & sonar interpretation \\
\hline (Tailor, 1988) & MXA & data monitoring \\
\hline (Dai et al., 1990) & HOPES & real-time \\
\hline (Mulder \& Braspenning, 1988) & & radar interpretation \\
\hline (Lesser \& Corkill, 1983) & DVMT & vehicle monitoring \\
\hline (Gallestigni et al., 1989) & - & $\begin{array}{l}\text { real-time sound } \\
\text { classification }\end{array}$ \\
\hline (Boureau \& Hayes-Roth, 1989) & GUARDIAN & patient monitoring \\
\hline (Kaiser et al., 1988) & PVIM & $\begin{array}{l}\text { cockpit information } \\
\text { manager }\end{array}$ \\
\hline (Pomeroy \& Irving, 1990) & $\begin{array}{l}\text { Pilot's } \\
\text { Associate }\end{array}$ & pilot assistance \\
\hline (Rubin et al., 1988) & OFMSPERT & operator assistance \\
\hline
\end{tabular}

Table 2.5: Monitoring over time applications.

Monitoring over time applications clearly exploit the same characteristics of the blackboard architecture as interpretation tasks. Additionally, these applications can make use of a blackboard architecture in two other ways. The blackboard can be used to represent and maintain the current interpretation of the domain under observation. The event-driven character of the blackboard architecture can be used to integrate and react to newly arriving data. Table 2.5 presents several examples of blackboard applications for monitoring over time tasks.

The first blackboard systems for this kind of task were HASP and SIAP, which are architecturally sufficiently similas to be referred to as one system ( $\mathrm{Nii}$ et al., 1988). HASP/SIAP - also known as SU/X (Nii \& Feigenbaum, 1978) - had as its domain monitoring and interpretation of sonar data from an area of deep ocean to detect, localise, and classify vessels within the area. Building on HASP/SIAP were the blackboard framework MXA for data monitoring tasks (Stammers, 1985; Tailor, 1988) and the concurrent blackboard system HOPES for real-time radar signal interpretation and processing (Dai et al, 1990). Mulder and Braspenning used MXA to develop a blackboard system for recognising and classifying possibly moving objects in a three dimensional environment, based on two-dimensional radar images (Mulder \& Braspenning, 1988). 
Two applications use sound as a source of information for monitoring purposes. These are the Distributed Vehicle Monitoring Testbed (DVMT, Lesser \& Corkill, 1983) and a test application on top of GBB (Corkill et al, 1986) for real-time sound classification (Gallestigni et al., 1989). DVMT is a distributed blackboard architecture primarily developed for distributed AI research. Its application is tracking of vehicles based on sounds.

GUARDIAN (Boureau \& Hayes-Roth, 1989) is a real-time development on top of BB1 (Hayes-Roth, 1985) for monitoring patients.

Airplane pilots often have large amounts of data at their disposal. Tools for monitoring and preprocessing this information have been developed using the blackboard architecture. PVIM (Kaiser et al., 1988) is a system for cockpit information management built on top of the two blackboard frameworks GBB (Corkill et al., 1986) and Erasmus (Baum et al., 1989). Pilot's Associate is developed for a similar application (Pomeroy \& Irving, 1990). OFMspert (Rubin et al., 1988; Jones et al., 1990) is a framework for assistance of operators of complex dynamic systems in general.

\subsubsection{Planning and configuration}

Planning and configuration form a family of related task domains. In all these problem areas the solution of a problem consists of a construction, using elementary building blocks, which satisfies as well as possible a number of prespecified constraints. In planning, the elementary building blocks are activities, which, when applied in a correct order, constitute a plan to reach a prespecified goal. Finding a solution is basically a search problem in which the solution is found among all possible constructions of all available buillding blocks.

The blackboard architecture can be utilised in these kinds of task domains in a number of ways. The blackboard can be used to store (possibly mutually inconsistent) partial solutions. Knowledge about how and when to create, expand, modify, combine, or discard partial solutions can be stored conveniently in different modules and applied opportunistically. An illustration of this was presented in Section 2.5.2, where a blackboard-based cognitive model for planning and its implementation OPM (Hayes-Roth \& Hayes-Roth, 1979) was discussed. 


\begin{tabular}{|c|c|c|}
\hline reference & name & application \\
\hline $\begin{array}{l}\text { (Hayes-Roth \& } \\
\text { Hayes-Roth, } 1979\end{array}$ & OPM & errand planning \\
\hline (Lutze, 1988) & LUPINO & $\begin{array}{l}\text { office automation } \\
\text { planning }\end{array}$ \\
\hline (Ciscon \& Johnsion, 1990) & - & robot path planning \\
\hline (Findler \& Gao, 1987) & - & $\begin{array}{l}\text { plant combine } \\
\text { production planning }\end{array}$ \\
\hline (Lumley, 1983) & PROBE & planming \\
\hline (Potter \& Lawrence, 1987) & TABULA & $\begin{array}{l}\text { military planning, } \\
\text { assessment, decision }\end{array}$ \\
\hline (Lehnert, 1989) & SARGE & $\begin{array}{l}\text { tactical decision aid in } \\
\text { simulated battle }\end{array}$ \\
\hline (Engelmore \& Terry, 1979) & Crysalis & $\begin{array}{l}\text { structures from } \\
\text { crystallographic data }\end{array}$ \\
\hline (Hayes-Roth et al, 1986a) & PROTEAN & $\begin{array}{l}\text { Protein structures from } \\
\text { constraints }\end{array}$ \\
\hline (Liempd et al., 1990) & Blondie-IIII & network configuration \\
\hline
\end{tabular}

Table 2.6: Planning and configuration applications.

Other examples of blackboard applications in a plamning domain are office automation planning (LUPINO, Lutze, 1988) and robot path planning (Ciscon \& Johnson, 1990). In (Findler \& Gao, 1987) a blackboard system is described for planning and controlling the combined production of various items by a distributed network of diverse production units in a plant. See Table 2.6 for an overview of these applications.

Over the years several blackboard frameworks dedicated to planning tasks have been developed. An example is the Prolog-based blackboard framework PROBE (Lumley, 1983). TABULA (Potter \& Lawrence, 1987) and SARGE (implemented in the blackboard shell GBB, Lehnert, 1989) are blackboard frameworks for military planning, assessment, and decision making.

Crysalis (Engelmore \& Terry, 1979; Terry, 1988) and PROTEAN (HayesRoth et al, 1986a) are two blackboard systems for chemical domains. Both try to determine structures of objects from experimental data. Crysalis (sometimes also refered to as $S U / P$ ) is an early blackboard system for determining structures from crystallographic data (Engelmore 
\& Terry, 1979). PROTEAN is a system for finding protein structures from constraints. In both applications the basic building blocks for a solution are more or less known. The problem is solved when a structure of building blocks has been found that is consistent with the data. In this respect these two applications can be considered as configuration applications.

To conclude this section, we mention the distributed blackboard framework Blondie-III that has been applied to a telecommunications network configuration problem (Liempd et al., 1990, see also Chapter 5).

\subsubsection{Control}

As seen earlier, the blackboard architecture can be used in an eventdriven manner. This aspect of the blackboard architecture can be exploited for control tasks, as control is concerned primarily with reaction to observed situations and events in the domain to be controlled. The applications in this section are presented also in Table 2.7.

In (Adey, 1988) application of a blackboard and events in a knowledgebased system for control of ships and aircraft is described. Air traffic control has been selected as application areas of the two distributed blackboard architectures CASSANDRA (Craig, 1989) and DISPROS (Saxena et al., 1990).

Examples of application of the blackboard architecture to robot control are the following: CODGER, a parallel blackboard architecture for robot navigation (Shaler \& Stentz, 1987), Blondie-II, a parallel blackboard shell developed mainly for robot cell control (Velthuijsen et al., $1987 \mathrm{~b}$, see also Chapter 4), and BEDS, a blackboard system for mission planning for an autonomous vehicle (Pearson, 1988). Additional examples can be found in (Pang, 1989; Harmon, 1989).

The blackboard system generator language BSG has been applied to control tasks also, i.e., to real-time satellite command and control and project management (Silverman et al, 1989). BBF is yet another blackboard framework developed for control tasks and has been applied in the areas of PABX maintenance and electricity network maintenance (Arlabosse et al, 1988). The parallel blackboard architecture HCVM has been applied to the control of real-time materials composition process (Fehling et al., 1989). BB-SPACE (Mathe, 1990) is an application of the 


\begin{tabular}{|c|c|c|}
\hline reference & name & application \\
\hline (Adey, 1988) & - & $\begin{array}{l}\text { ship and aircraft } \\
\text { control }\end{array}$ \\
\hline (Craig, 1989) & CASSANDRA-II & airspace control \\
\hline (Saxena el al., 1990) & DISPROS & air traffic control \\
\hline (Shafer \& Stentz, 1987) & CODGER & robot navigation \\
\hline (Velthuijsen et al, 1987b) & Blondie-II & robot cell control \\
\hline (Pearson, 1988) & BEDS & mission planning \\
\hline (Pang: 1989) & - & robot control \\
\hline (Harmon, 1989) & - & rabot control \\
\hline (Silverman et al., 1989) & BSG & $\begin{array}{l}\text { real-time satellite } \\
\text { command and } \\
\text { control }\end{array}$ \\
\hline (Arlabosse et al., 1988) & $\mathrm{BBF}$ & $\begin{array}{l}\text { PABX and electri- } \\
\text { city network } \\
\text { maintenance }\end{array}$ \\
\hline (Fehling et al., 1989) & HCVM & real-time control \\
\hline (Matlue, 1990) & $\mathrm{BB}-\mathrm{SPACE}$ & $\begin{array}{l}\text { operator assistance } \\
\text { in space telemani- } \\
\text { pulation }\end{array}$ \\
\hline (Valkonen et al, 1987) & $\mathrm{ADP}$ & language parsing \\
\hline (Niyogi \& Srihari, 1987) & - & $\begin{array}{c}\text { document image } \\
\text { understanding }\end{array}$ \\
\hline (Coben et al., 1989) & HITS & $\begin{array}{l}\text { human-computer } \\
\text { interface mana- } \\
\text { gement }\end{array}$ \\
\hline
\end{tabular}

Table 2.7: Control applications. 
blackboard shell ATOME (Laasri et ol., 1988b) to act as an operator assistant in space telemanipulation. In (Valkonen et al., 1987) the system ADP (Augmented Dependency Parser) is described. ADP uses a blackboard architecture for controlling a language parsing process. Similarly, Niyogi and Srihari describe a system for document image understanding where aspects of blackboard control are used to direct the image understanding process (Niyogi \& Srihari, 1987). Another system that uses a blackboard architecture for controlling subprocesses is the HITS system for human-computer interface management (Cohen et al., 1989).

\subsubsection{Integration}

The blackboard architecture has been used in occasions where diverse components needed to be integrated (see also Table 2.8. This varies from an integration of multiple sources of expertise (as was the case for instance in the speech understanding system Hearsay-II), via integration of different knowledge representation and inferencing techniques, to integration of different kinds of processing units. Integration in blackboard systems makes use of the strictly modular design of the architecture and its unified communication facilities between the modules via the blackboard. An example of integration of different kinds of processing units is given by the parallel blackboard framework KIM (Knowledge-based Integration Manager, Winkelbauer, 1988) for the integration of symbolic and numerical processing units. Similarly, in (Märtin \& Waldhör, 1988) the use of a blackboard for integrating standard and knowledge-based applications in an office automation domain is presented.

The modularity of the blackboard architecture has also been exploited for integration of multiple sources of expertise. The system DFI (Designer Fabricator Interpreter, Werkman \& Hillman, 1989) is developed to facilitate communication between designers, fabricators, and erectors who need to cooperate on the production of steel constructions. DFI uses a blackboard for communication between modules containing expertise related to the different specialisations in the process leading from design to production. In (Fernandez de Castro et al., 1988) an intelligent computer aided instruction system is described which uses a blackboard architecture to integrate components specialised in different aspects of the tutoring task. 


\begin{tabular}{|c|c|c|}
\hline reference & name & application \\
\hline (Winkelbauer, 1988) & $\mathrm{KIM}$ & $\begin{array}{l}\text { integrating coupled } \\
\text { systems }\end{array}$ \\
\hline (Martin \& Waldhör, 1988) & Basar & office automation \\
\hline (Werkman \& Hillman, 1989) & DFI & $\begin{array}{l}\text { designer fabricator } \\
\text { interpreter }\end{array}$ \\
\hline $\begin{array}{l}\text { (Fernandez de Castro } \\
\text { et al., 1988) }\end{array}$ & - & IOAI \\
\hline (Milne \& Eldridge, 1988) & RARE & $\begin{array}{l}\text { deep model } \\
\text { integration }\end{array}$ \\
\hline (Sriram, 1987) & DESTINY & structural design \\
\hline
\end{tabular}

Table 2.8: Integration applications.

The RARE system (Reasoning About Relationships, Milne \& Eldridge, 1988) extends the techniques for building second generation expert systems using 'deep models' (Chandrasekaran \& Mittal, 1983) and 'modelbased reasoning" (Davis \& Hamscher, 1988), by providing a means for integrating several models for its application domain. RARE uses a blackboard to integrate these models and a control component to decide which model to use in a given situation.

DESTINY is a blackboard framework for integrating different stages in a structural design process (Sriram, 1987).

\subsubsection{Development environment}

The blackboard architecture provides great flexibility with respect to addition, removal, or replacement of knowledge sources and with respect to control. As a consequence, the blackboard architecture can be useful in domains in which an optimal approach to solving problems is not yet clear. It may not be known at the start of development of an application. system how the overall problem can be divided into a number of subproblems, how these subproblems should be solved, or in what order subproblems should be tackled. Then, the flexibility of a blackboard architecture may be used to experiment with different approaches until a good ajpproach is identified. Erman and Lesser have given some attention to these and similar issues regarding system engineering techniques in the 
context of system development of the Hearsay-II speech-understanding system (Erman \& Lesser, 1978). Additional problems with the original development of a speech-understanding system addressed by the design of Hearsay-II were posed by the involvement of researchers from various fields of expertise and by the great complexity and size of the eventual system.

When a blackboard architecture has been used for the development of a system primarily for reasons of experimentation, it might be a good decision to remove the cost of flexibility by reimplementing the system. However, this move is only plausible when the system does not exploit significantly any other characteristic feature of the blackboard architecture. In (McCracken, 1979) an attempt is described to transfer the knowledge in the Hearsay-II system to a production-rule system. Although it proved to be possible to perform the transfer without loss of expressiveness, the production-system implementation showed substantial problems regarding space and time efficiency resulting in a loss of time efficiency of at least two orders of magnitude. This phenomenon is due to the fact that significant portions of control and working memory in a blackboard architecture can remain local within the knowledge sources and be optimised for each knowledge source separately. The discussion of characterising features of the blackboard architecture in Section 7.3 presents other indications why some blackboard systems would lose much of their effectiveness when reimplemented as a "nonblackboard' system.

\subsection{Blackboard frameworks and generators}

After the listing of blackboard-system applicalions in the previous section, we concentrate now on domain-independent blackboard architectures. Such architectures can be used to develop blackboard applications by adding domain-dependent information. A distinction is made between blackboard frameworks and blackboard generators. Blackboard frameworlss are computer systems which provide the functionality of elements of a blackboard architecture that can be used to implement an application. Typically, a blackboard framework, together with an application, contains the complete functionality provided by the framework, including functionality not used by the application. This can make the 


\begin{tabular}{|l|l|}
\hline reference & name \\
\hline (Balzer et al, 1980) & Hearsay-III \\
(Nii \& Aiello, 1979) & AGE \\
(Tailor, 1988) & MXA \\
(Aiello, 1986) & CAGE \\
(Rice, 1986) & POLIGON \\
(Hayes-Roth, 1985) & BB1 \\
(Corkill et al., 1986) & GBB \\
(Craig, 1986) & Ariadne-I \\
(Craig, 1989) & CASSANDRA \\
(Haton et al., 1987) & ATOME \\
(Lumley, 1983) & PROBE \\
(Jones et al., 1986) & Edinburgh \\
(Roth et al., 1987) & GEST \\
(Fikes, 1987) & OPUS \\
(Lippolt et al., 1987) & Blondie \\
(Velthuijsen et al., 1987b) & Blondie-Il \\
(Liempd et al., 1989) & Blondie-III \\
(Baum et al., 1989) & Erasnus \\
(Silverman et al., 1989) & BSG \\
(Levy \& Finin, 1987) & BBC \\
(Brajnik et al., 1988) & ADL \\
\hline
\end{tabular}

Table 2.9: Frameworks and generators.

resulting system unnecessarily large and inefficient. To overcome this problem a blackboard generator provides a specification environment for describing precisely the blackboard-architecture functionality needed for a certain application. The description is compiled, together with the domain code, to create a blackboard system with a tailored blackboard architecture.

An overview of the blackboard frameworks and generators mentioned in this section can be found in Table 2.9.

\subsubsection{Blackboard frameworks}

The earliest blackboard frameworks emerged as generalisations of blackboard systems. Examples are Hearsay-III (Balzer et al., 1980), a gen- 
eralisation of Hearsay-II (Erman et al., 1980), and AGE (Nii \& Aiello, 1979), which was a generalisation and extension of experiences with the HASP/SIAP system (Nii et al., 1988). Both frameworks are described extensively in Appendix A of this thesis. Also based on HASP/SIAP is the MXA shell (Stammers, 1985; Tailor, 1988), developed primarily for data monitoring tasks.

CAGE (Concurrent AGE, Aiello, 1986) and POLIGON (Rice, 1986; Rice, 1989b) are two distinct parallelisation efforts of the blackboard architecture by the same group of researchers at Stanford University. CAGE is a parallel version of $A G E$. The developer of an application in CAGE needs to specify when and where opportunities for concurrency should be exploited. POLIGON, on the other hand, uses an opposite approach: activities are executed in parallel unless the application builder specifies differently. These two frameworks are described in Appendix A as well.

The blackboard framework BB1 (Hayes-Roth, 1985; Appendix A) was developed as a generalisation of the opportunistic planning system OPM (Hayes-Roth \& Hayes-Roth, 1979). Similarities between control in blackboard systems and opportunistic planning led to an architecture with an emphasis on control. Some of the higher abstraction levels of the OPM planning system return in BB1 as a control component. Experience with different applications in $\mathrm{BB} 1$ led to the idea of creating several blackboard-framework environments, each based on BBI and tailored to a different category of applications. The set of environments is called BB* (Hayes-Roth et al, 1986b) and includes the ACCORD environment for plaming and configuration tasks. Two applications in the ACCORD environment are a reimplementation of PROTEAN (see earlier) and SIGHTPLAN (Tommelein el al., 1987) for the design of construction site layouts.

GBB (Generic Blackboard framework, Corkill et al., 1986; Appendix A) was developed at the University of Massachusetts. The design and implementation of GBB benefitted from the availability of experience with the development of the earlier blackboard architectures Hearsay-II ( $E r-$ man el al, 1980; Appendix A) and DVMT (Lesser \& Corkill, 1983; Appendix A). GBB focuses on flexibility and efficiency of blackboard storage and access.

Craig has developed three frameworks: Ariadne-I (Craig, 1986), mod- 
elled after Hearsay-III and BB1, NBB (Craig, 1987a), and CASSANDRA (Craig, 1989; Appendix A). CASSANDRA is a distributed blackboard framework. Each node in this distributed framework consists of a blackboard level, a set of knowledge sources and a Level Manager containing a control component and facilities for communication with other nodes.

The framework ATOME (Haton et al., 1987), resembling AGE with respect to its control aspects, has been used for research into some developmental and organisational issues (Laasri et al., 1988b; Laasri et al., 1988a; Brunessaux \& Chevrier, 1990) as well as multi-agent architectures (Gong \& Haton, 1989).

Most early blackboard frameworks were implemented in LISP. PROBE (Prolog Blackboard Environment, Lumley, 1983) and the Edinburgh Prolog Blackboard Shell (Jones et al., 1986) proved that blackboard frameworks could be implemented successfully in Prolog.

The blackboard architecture has been added in some cases as a tool to more general expert system building tools. The framework GEST (Generic Expert System Tool, Roth et al., 1987; Tynor et al., 1987) is an expert system shell which also includes a blackboard component. In (Fikes, 1987) OPUS is described. OPUS integrates a blackboard framework in the expert-system tool $\mathrm{KEE}^{5}$.

In this thesis we also present three blackboard frameworks developed by ourselves. Blondie is a blackboard framework based on BB1. BlondieII extends the Blondie framework with parallel execution of knowledge sources. Blondie-III is a distributed blackboard framework.

\subsubsection{Blackboard generators}

We conclude this chapter by mentioning several blackboard generators. Erasmus (Baum et al., 1989) and BSG (Silverman et al., 1989) are both such generators which can be used both to specify and create parallel and distributed blackboard architectures. BBC (Blackboard Builder in C, Levy \& Finin, 1987) produces a blackboard system in the programming language $\mathrm{C}$.

\footnotetext{
"KEE is a trademark of IntelliCorp, Inc.
} 
The blackboard specification language ADL (Architecture Description Language, Brajnik et al., 1988) was developed not only for designing blackboard systems, but also for gaining a better understanding of the blackboard architecture. It was assumed that a precise study of the blackboard architecture resulting from the specification of components would lead to a more complete understanding of the architecture. This agrees with the ideas expressed in Chapter 6. 


\section{Chapter 3}

\section{The Blackboard Framework Blondie}

The blackboard framework Blondie was developed in the first half of 1986 as a means to gain a better understanding of the blackboard architecture. It appeared from several references that the blackboard architecture could provide a useful approach for creating complex expert systems. Then, the literature was not very specific in describing how to build such an architecture and what the characteristics of such an architecture would be. Building Blondie served two purposes. Firstly, by building a blackboard framework and applying it to a test application, we gained a better understanding of the available literature. Secondly, we developed a tool for experimentation that could be used to check claims in the literature and to determine advantages and disadvantages of using $a$ blackiboard architecture.

Blondie is based primarily on BB1. The control unit in $B B 1$ is described explicitly in (Hayes-Roth, 1985), thus providing useful guidelines for the implementation of a blackboard architecture. Blondie is a blackboard framework and contains neither domain-specific "knowledge" nor domainspecific components. The framework Blondie can be used for building sequential blacliboard systems and provides procedures for defining and accessing a multiple-panel blackboard, procedures for defining and handling knowledge sources and a control unit, and facilities for inspecting and tracing the behaviour of the framework when running an application. 
Our test application tries to solve a number game. The input consists of a set of six numbers and one additional seventh number: the target number. The task is to find a way to calculate or approximate the target number as closely as possible within the allotted time, by using each of the six numbers at most once and combining them using the four arithmetic operators + , ,$- x$, and /. An example:

six numbers: $\quad 100,25,10,9,3,2$

target number: 584

approximation: $\quad 584=100 \times\left(\frac{10}{2}\right)+25 \times 3+9$

The test application implements a number of different heuristic strategies for solving such a puzzle, where the choice for a specific strategy may depend on characteristics of the given numbers. One such strategy, which can be applied when there is a "large' number among the six, divides the target number by that large number, resulting in a remainder and a quotient. When the quotient can be calculated and the remainder approximated using the remaining numbers, an approximation to the target number has been found.

Figure 3.1: Description of the number game.

Blondie was written in the programming language Pop-11 (Barrett et al., 1985), part of the AI programming environment Poplog. Pop-11 provides facilities for list processing, similar to Lisp, but has a Pascal-like syntax. Besides Pop-11 Poplog contains the languages Prolog, Lisp, and $\mathrm{ML}$ (a functional programming language), as well as facilities for mixing within one program code written in any of these different languages. It is allso possible in Poplog to incorporate calls to procedures or functions written in external programming languages, such as $\mathrm{C}$ or Fortran. Furthermore, Poplog contains facilities for object-oriented programming. Although the action parts of knowledge sources (KSs) must have an interface written in Pop-11, the versatility of the Poplog programming environment allows one to use different programming languages and programming techniques, such as rule-based programming; within knowledge sources.

This chapter presents the blackboard framework Blondie. A simple test application is used to illustrate its features. This test application tries to solve a number game, which is explained in Figure 3.1. The test applica- 
tion is not meant to illustrate the power of the blackboard architecture, but only to illustrate how Blondie is designed and how an application can be specified.

Section 3.1 describes Blondie. Section 3.2 presents an evaluation of Blondie. The material in this chapter is based on an earlier report (Lippolt et al., 1986). More information about the functionality of Blondie can be found in its manual (Velthuijsen, 1987c). An overwiew of Blondie has been published in (Lippolt et al., 1987).

\subsection{Blondie}

The description of Blondie is subdivided into four separate sections, one each for:

- the blackboard,

- the knowledge sources,

- the control unit, and

- the interface.

Blondie consists of a number of modules, each containing procedures relevant for handling a certain kind of objects in a blackboard system, such as the blackboard, events, or knowledge sources. This subdivision is shown in Figure 3.10. The functionality of the modules is explained throughout the text. The modules are grouped together in Figure 3.10 to give an impression of the calling chain: a procedure in one module can only call procedures in modules in the same or lower groups as indicated by the arrows.

\subsubsection{Blackboard}

\section{Representing data on the blackboard}

The blackboard in Blondie can be subdivided in the following way. The blackboard can be subdivided into panels. In turn, a panel is divided. into several blackboard levels. On each level, data elements are stored 


\begin{tabular}{|ll|}
\hline NAME & three1 \\
NUMBER & 390 \\
USED-ELMNTS & {$[$ basis5 basis2 basis6 $]$} \\
PROOF & $(15 *(2 * 13))$ \\
UPLINKS & $[$ four $]]$ \\
\hline
\end{tabular}

Figure 3.2: Example of a blackboard unit.

in units, as we call them. In Blondie, these units are implemented as lists of attribute-value pairs. All units on the same level have the same attributes, although the corresponding values may be 'undefined'. The panels, levels, and attributes for units on each level are statically defined. Essentially, blackboard units on the same level have the same 'type'.

Figure 3.2 shows an example of a unit. The name of a unit is formed by concatenating the name of the blackboard level on which the unit is placed (in this case 'three') and a number indicating the sequential order of creation of units on that same blackboard level. In our test application the unit in Figure 3.2 represents the number ' 390 ', which has been calculated by using three (hence the name of the level) of the original six numbers. The blackboard units representing these three numbers are referenced by the attribute USED-ELMNTS. PROOF indicates the calculation yielding ' 390 '. This unit has been used in another calculation to form a unit on blackboard level 'four', as specified by UPLINKS.

The test application contains two blackboard panels: one is called control blackboard, the other domain blackboard (see Figure 3.3 for an overview of the panels and levels). The control blackboard is used by the control unit and contains information needed for selecting knowledge sources. The domain blackboard contains information directly pertaining to solving the number game. Figure 3.4 illustrates the contents of the control blackboard at the end of a problem-solving session. Figure 3.5 shows parts of the domain blackboard for the same problem at the same time.

The control blackboard (see also Figure 3.4) and two of its levels, todo-set and chosen action, must be present in all applications of Blondie. The blackboard level to-do-set contains information about all knowledge source that have not yet been executed, and states whether each instantiation is applicable in the current state. The level chosen-action 


\begin{tabular}{|l|l|l|}
\hline domain & control \\
\hline SIX & TO-DO-SET & \\
FIVE & CHOSEN-ACTION & \\
FOUR & PROBLEM & \\
THREE & STRATEGY & \\
TWO & & \\
BASIS & & \\
ARITHM & & \\
\hline
\end{tabular}

Figure 3.3: Panels and levels in the test application.

contains information about the knowledge source instantiations that are selected consecutively. In the test application the blackboard level problem describes the problem to be solved, i.e., the target number (the six other numbers are represented on the domain blackboard panel). Also, the best current solution is stored here, as well as information about whether a solution has already been printed or whether the allotted time has expired. Level strategy is used to store descriptions of heuristic strategies to be used during the problem-solving process. These descriptions are presented in terms of numbers that must be calculated (determined, attribute TO-BE-DET) or approximated (attribute TOBE-APPR) and the numbers that can be used for either activity (DETWITH and APPR-WITH, respectively). The attribute BIG-STEP indicates a number to be used for approximating the target number. Usually, the largest or second largest number of the six originally supplied numbers is used (e.g., ' 100 ' in the approximation in Figure 3.1). However, BIG-STEP can be formed as the sum or product of two numbers as well. This is included in the test application, but specified by strategy-units not shown in Figure 3.4.

The domain blackboard panel stores descriptions of derived calculations, which are used for reconstructing the actual solution for printing results (see Figure 3.5). The attributes are used as described for Figure 3.2. Additionally, blackboard level arithm is used for storage of descriptions of calculations of numbers that must be added to the domain blackboard. Not all calculated numbers are written on the blackboard, as it would lead to excessive blackiboard access resulting in efficiency problems. ${ }^{1}$

\footnotetext{
${ }^{1}$ In our original implementation we did write all intermediately generated numbers on the blackboard. We changed the implementation (without much effort), when we observed that this approach did not meet the time constraints of the number game.
} 


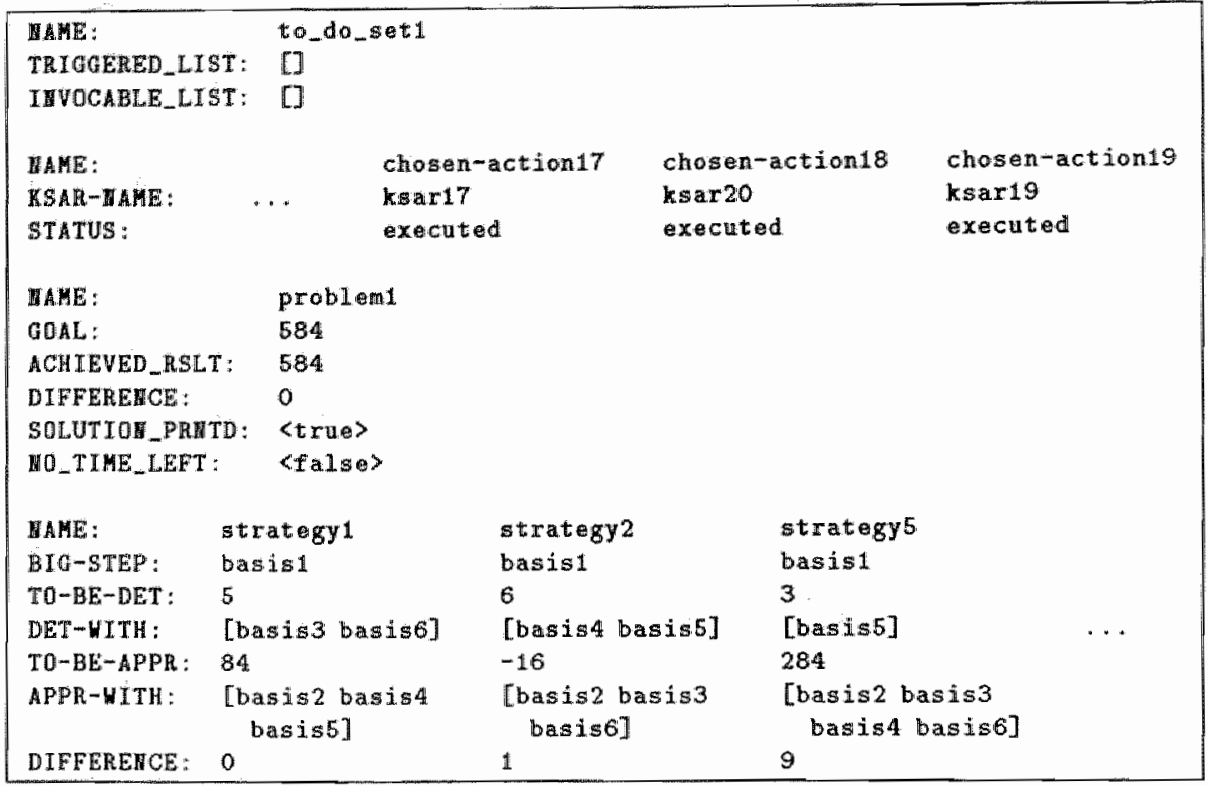

Figure 3.4: Example of the control blackboard panel.

While a control blackboard with levels to-do-set and chosen-action must be present in each application of Blondie, the control blackboard may contain additional levels as seen in the example. An application may contain as many panels and levels on these panels as deemed necessary. Blondie does not support the explicit use of links (objects representing relations between different blackboard units). However, as illustrated in Figure 3.2 , links can be represented as attributes of units. An application builder must implement procedures for exploiting these links and for maintaining consistency.

\section{Writing to and reading from the blackboard}

The blackboard and procedures for accessing and handling the blackboard form a module. This module is called the blackboard-handler (or bb-handler for short). The bb-handler contains procedures for the following:

- Initialisation of the blackboard panels and blackboard levels and definition of allowed unit attributes on each level. 


\begin{tabular}{|c|c|c|c|c|c|}
\hline IAME: & \multicolumn{2}{|l|}{$\operatorname{six} 1$} & \multicolumn{2}{|l|}{$\operatorname{six} 2$} & \\
\hline IUHBER: & \multicolumn{2}{|l|}{584} & \multicolumn{2}{|l|}{583} & \\
\hline PRODF: & \multicolumn{2}{|c|}{$(100 *(10 / 2))+(9+(25 * 3))$} & \multicolumn{3}{|c|}{$(100 *(9-3))-(25-(10-2))$} \\
\hline BAME: & \multicolumn{5}{|l|}{ five1 } \\
\hline IUKBER: & \multicolumn{5}{|l|}{575} \\
\hline PROOF: & \multicolumn{5}{|c|}{$(100 * 3)+(25 *(9+2))$} \\
\hline UPL INKS : & \multicolumn{5}{|c|}{$\left[\begin{array}{l}{[]} \\
\text { [basis1 basis } 5 \text { basis? }\end{array}\right.$} \\
\hline USED-ELMETS: & \multicolumn{5}{|c|}{$\begin{array}{l}\text { [basis } 1 \text { basis } 5 \text { basis } 2 \\
\text { basis } 4 \text { basis } 6 \text { ] }\end{array}$} \\
\hline \multirow{5}{*}{\multicolumn{6}{|c|}{$\begin{array}{l}\text { IAME: } \\
\text { HUMBER: } \\
\text { PROOF: } \\
\text { UPLINKS: } \\
\text { USED-ELMATS: }\end{array}$}} \\
\hline & & & & & \\
\hline & & & & & \\
\hline & & & & & \\
\hline & & & & & \\
\hline MAME: & three 1 & three? & & three 3 & \\
\hline YUMBER: & 500 & 84 & & 600 & \\
\hline PROOF : & $100 *(10 / 2)$ & $9+(25 * 3)$ & & $100 *(9-3)$ & \\
\hline UPLINKS: & {$[s i x 1]$} & {$[\sin 1]$} & & {$[\operatorname{six} 2]$} & \\
\hline USED-ELNITS : & $\begin{array}{l}\text { [basis } 1 \text { basis } 3 \\
\text { basis } 6]\end{array}$ & $\begin{array}{r}\text { [basis } 4 \\
\text { basis } 5\end{array}$ & $\begin{array}{l}\text { basis2 } \\
\text { ] }\end{array}$ & $\begin{array}{l}\text { [basis1 basis } 4 \\
\text { basis5] }\end{array}$ & \\
\hline NAME: & troo 1 & $t w 02$ & & tro3 & \\
\hline MUMBER: & 5 & 75 & & 6 & \\
\hline PROOF : & $10 / 2$ & $25 * 3$ & & $9-3$ & \\
\hline UPLTNRS: & [three1] & [three 2$]$ & & [xive3] & \\
\hline USED-ELMUTS: & [basis 3 basis6] & [basis 2 & basis 5] & [basis4 basis5] & \\
\hline NAME: & basis3 & basist & & basis 5 & \\
\hline MUMBER: & 10 & 100 & & 25 & \\
\hline PRQOF : & 10 & 100 & & $\cdots$ & \\
\hline UPLINKS: & 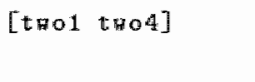 & $\begin{array}{l}{[\text { threed }} \\
\text { two5] }\end{array}$ & three 3 & $\begin{array}{l}{[\mathrm{t} \text { t*2 } \mathrm{t} \text { - } 0.3} \\
\text { tro5] }\end{array}$ & \\
\hline MAME: & arithm1 & & arithm & & \\
\hline COMPTNDM: & $\begin{array}{l}\text { [basis } 1 \text { multiply } \\
\text { [basis } 3 \text { divide }\end{array}$ & basis6]J & $\begin{array}{l}\text { [three } \\
\text { [bas: }\end{array}$ & $\begin{array}{l}1 \text { plus [basis } 4 \text { plus } \\
\text { is } 2 \text { mult iply basis5]]] }\end{array}$ & . \\
\hline
\end{tabular}

Figure 3.5: Example of the domain blackboard panel. 
- Creation of a unit on a blackboard level. Whenever a new unit is created, attributes are given the values specified in the procedure call, or the value 'undefined' if no value was specified. A unique name for the unit is automatically generated and returned. Thus, the procedure call

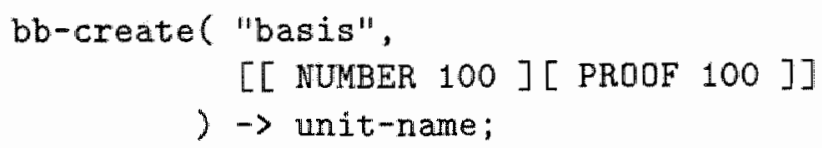

results in creation of a unit that looks like:

$\begin{array}{ll}\text { NAME } & \text { basis1 } \\ \text { NUMBER } & 100 \\ \text { PROOF } & 100 \\ \text { UPLINKS } & \text { undefined }\end{array}$

- Modification of a unit. This procedure can be used to modify any attribute or collection of attributes of a unit, except the name of that unit.

- Searching the blackboard for units corresponding with a specific description, essentially using a pattern matching procedure. As an example, the following procedure call

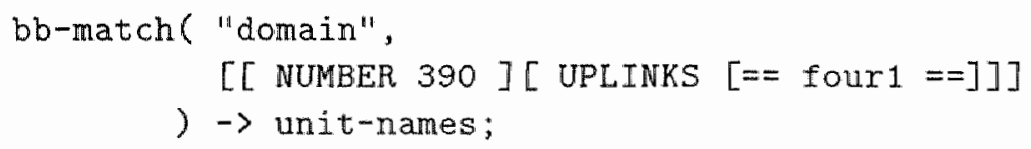

yields the names of all units on the domain blackboard panel with value '390' for attribute NUMBER and with 'four 1' in its list of UPLINKS (the symbol ' $==$ ' indicates a don't care option).

- Printing one specific unit, all units on a blackboard level, or all units on a blackboard panel.

\section{Events}

Each time a unit on the blackboard is created or an attribute of a unit is modified, an event is created. Events specify the occurrence of changes 


\begin{tabular}{|ll|}
\hline KIND & modifed \\
BLACKBOARD & control \\
BLACKBOARD-LEVEL & strategy \\
UNIT-ID & strategy3 \\
ATTRIBUTE-ID & difference \\
\hline
\end{tabular}

Figure 3.6: Example of an event.

on the blackboard and are used to determine which knowledge sources could take part in the problem-solving process at any given moment. Events are also implemented as a list of attribute-value pairs. An example of an event is shown in Figure 3.6. Blondie distinguishes between two kinds of events, signalling the creation of new units or the modification of existing units. This is specified in an event by the value 'new' or 'modified' for attribute KIND. An event further specifies the blackboard panel (BLACKBOARD), level (BLACKBOARD-LEVEL), and unit name (UNIT-ID) involved in the change. Events of kind 'modified' also contain a reference to the modified attribute (ATTRIBUTE-ID).

Procedures for handling events are collected in the module event-handler (or e-handler). The e-handler provides procedures for the following tasks:

- Creation of events upon blackboard modifications.

- Producing all events created during the last cycle of the control loop.

- Producing ali events created during the entire in of the system.

- Printing events.

\subsubsection{Knowledge sources}

Knowledge sources (KSs) in Blondie are composed of two distinct parts. The first part, called the KS descriptor consists of attribute-value pairs and contains information necessary for determining applicability of KSs and for selecting $\mathbb{K S}$ for execution. The second part is the actual action part of the KS and contains the code (with a Pop- 11 procedure as 
interface, possibly implemented using other programming languages or programming techniques) by which that $\mathrm{KS}$ actually contributes to the problem-solving process. The action parts can access the blackboard to write to and read from the blackboard.

A KS can be instantiated when an event is generated matching the condition specified in the $\mathrm{KS}$ descriptor. Instantiation results in the creation of a data structure, called a knowledge source activation record, containing information about the $\mathrm{KS}$ and the event involved in the instantiation. KS descriptors and knowledge source activation records (KSARs) are described separately in the following two sections. The description of KS descriptors and KSARs is followed by a discussion of KSs in our test application.

\section{KS descriptors}

An example of a KS descriptor is shown in Figure 3.7. The NAME and DESCRIPTION attributes of a KS descriptor are used to provide meaningful references to the KSs for users of Blondie. The TRIGGER specifies the events of interest to the KS. A KSAR is created by the control unit each time an event is generated matching the TRIGGER. Thus, in this example, a KSAR is created for KS basis-strategy each time an event is generated containing the attribute-value pairs:

\section{[BLACKBOARD-LEVEL problem] and [KIND new],}

i.e., whenever a unit on blackboard level problem is created. A trigger can be general, e.g., specifying that an instantiation should be created as soon as a unit has been created on the blackboard, or more specific, e.g., indicating that an instantiation is created only when a specified attribute of a certain unit is modified.

The value of attribute PRECONDITION denotes the name of a procedure. This procedure can be used to check whether a KS instantiation is actually applicable. A trigger alone is not always precise enough to determine applicability of KS instantiations. Therefore, an additional test may be necessary. This test is implemented in the precondition procedure. Thus, the condition of a KS is subdivided into two parts (the trigger and the precondition). The trigger specifies blackboard changes 


\begin{tabular}{|lll|}
\hline NAME & basis-strategy \\
DESCRIPTION & This KS creates descriptions of several \\
& heuristic strategies for solving the problen \\
TRIGGER & BLACKBOARDLEVEL problem \\
& KIND & new \\
PRECONDITION & trivial & \\
VARIABLES & F & control \\
FEATURES & FROM-BB & control \\
& TO-BB & problem \\
& FROM-LEVEL & strategy \\
& TO-LEVEL & 0.9 \\
& EFFICIENCY & 0.8 \\
& RELIABILITY & generate \\
& KS-TYPE & \\
\hline
\end{tabular}

Figure 3.7: Example of a KS descriptor.

that have to occur before a KS can be executed, the precondition specifies additional information that must be present on the blackboard before a KS can become executable. Each KS can have its own precondition procedure.

Preconditions are used also to bind input variables for the action part of a KS to values specific to a certain instantiation. These variables are listed as value for attribute VARIABLES. A precondition procedure takes as its input the identification of the unit responsible for triggering and the value of VARIABLES. The output is a Boolean value, indicating whether the test succeeded, and attribute-value pairs for the variables with their bound values. A precondition procedure may use all information present on the blackboard. (The precondition procedure trivial in the $\mathrm{KS}$ descriptor in Figure 3.7 takes an empty list of variables and always produces true as result for the test and an emply list of bound variables.)

The attribute FEATURES has a list of attribute-value pairs as its value. This list contains information that can be useful for selecting KS instantiations for execution. The list of attributes can be defined by an application builder. The cxample in Figure 3.7 contains information about the blackboard panels and levels where information is read from and written to. It also contains an indication of efficiency and reliability of 


\begin{tabular}{|lll|}
\hline NAME & ksar21 & \\
KS-NAME & multiply & \\
TRIGGERING-CYCLE & 8 & \\
TRIGGERING-EVENT & BLACKBOARD-LEVEL & multiply \\
TRIGGERING-UNIT & multiply2 & \\
PRECONDITION & get-units & \\
VALUES & unit1 unit2 & arithmetic \\
FEATURE-VALUES & FROM-BB & domain \\
& TO-BB & multiply \\
& FROM-LEVEL & undef \\
& TO-LEVEL & 1.0 \\
& EFFICIENCY & 1.0 \\
& RELIABILITY & calculation \\
PRIORITY & KS-TYPE & \\
\hline
\end{tabular}

Figure 3.8: Example of a Knowledge Source Activation Record (KSAR).

the heuristic procedure implemented by that KS. Attribute KS-TYPE subdivides the set of KSs into subsets, each of which have their own selection priorities.

\section{Knowledge source activation records}

Whenever the trigger of a $\mathbb{K} S$ is matched by an event, Blondie creates a data structure describing the $\mathrm{KS}$ and the information that led to successful matching. An example of such a data structure, called a knowledge source activation record or $K S A R$, can be found in Figure 3.8. We note that it is possible that more than one KSAR exists for any KS at the same time, as new situations may occur, triggering the same KS again, before earlier instantiations have been executed.

As soon as a KSAR is created, such a KSAR is referred to as being triggered. A IKS instantiation becomes executable only when the corresponding precondition lias been executed successfully. After that, the KSAR is referred to as being invocable.

The attributes in a KSAR have the following meaning. NAME indicates the identification of a KSAR. These identifications are numbered accord- 
ing to the order of creation. KS-NAME contains the name of the corresponding KS. TRIGGERING-CYCLE, TRIGGERING-EVENT, and TRIGGERING-UNIT specify the sitwation existing at the time of triggering. TRIGGERING-CYCLE is a number indicating how many times the control unit has executed its loop (see also Section 3.1.3) and can be used for temporal ordering of events. TRIGGERING-EVENT is the same as TRIGGER in the KS descriptor. TRIGGERING-UNIT refers to the unit identification involved in the triggering. PRECONDITION is the same attribute as in the corresponding KS descriptor. VALUES contains values for each variable in the list associated with VARIABLES in the KS descriptor. The FEATURE-VALUES in this example have the same attributes as the KS descriptor in Figure 3.7. An application. builder can use and redefine these attributes as necessary.

The PRIORITY of a KSAR is calculated by the control unit. For this, the control unit uses a procedure provided by the application builder. This procedure is domain specific and can make use of all information available on the blackboard and in the KSAR.

Procedures for handling KSs and KSARs are collected in two modules, called $k s$-handler and $k s a r-h a n d l e r$, respectively. The $k s-h a n d l e r$ has procedures for the following activities:

- Execution of the action part of a KS.

- Producing a list of all KS descriptors.

- Producing narmes of KSs triggering on a given event.

- Printing IKS descriptors.

The procedures in the ksar-handler provide the following:

- Creation of KSARs.

- Modification of KSARs (especially to update priorities).

- Removal of KSARs from the set of currently available (triggered and invocable) KSARs.

- Producing the KSAR for a given KSAR identification.

- Printing of KSSARs. 


\section{start-up}

interacts with the user to select numbers (possibly via a random generator) and to set the allotted time.

\section{basis-strategy}

generates descriptions of a number of heuristic strategies; descriptions are defined in terms of numbers to be $\mathrm{cal}$ culated and numbers to be approximated and placed on blackboard level strategy.

\section{determine}

tries to calculate a given number from other given numbers.

\section{approximate}

approximates a given number using other given numbers.

\section{arithmetic}

records results of KSs determine and approximate on the domain blackboard panel for future reference.

\section{update-solution}

updates the representation of the current best approximation upon termination of a heuristic strategy.

\section{print-result1}

prints the best solution, when the allotted time has expired.

\section{print-result2}

prints the best solution, when no executable KSs are available anymore.

Table 3.1: Knowledge sources in the test application.

\section{Knowledge sources in our test application}

The KSs used in our test application are described in Figure 3.1. Figure 3.9 shows how KSs interact through blackboard access. This illustration contains the two blackboard panels control and domain and the levels on these panels. The arrows indicate what blackboard information is used primarily as input and where results are written on the blackboard. The names of the corresponding KSs are written in italics.

At the beginning of a problem-solving session the KS start-up interacts with the user to define the problem to be solved. A complete specification of the problem is split and stored on blackboard levels problem and basis. The creation of units on these levels triggers the $\mathbb{K} S$ basisstrategy which in turn creates a number of units on level strategy, each representing a different heuristic strategy for solving the problem. The creation of any unit on level strategy triggers the KS determine. (thus, for each heuristic strategy this KS is triggered once). KS determine tries to calculate the number specified by attribute TO-BE-DET. When such a calculation is found, a description of this is placed on level arithm and the strategy unit that triggered the KS is updated to specify which 


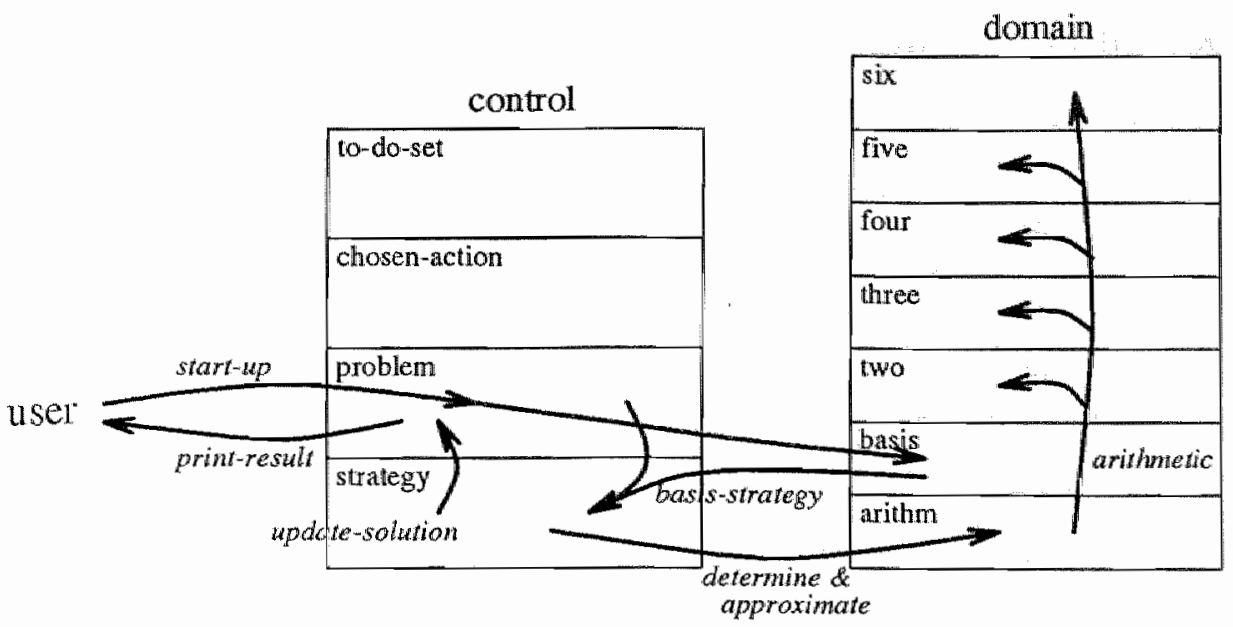

Figure 3.9: KS interactions in the test application.

numbers are left for approximation. This updating results in triggering of KS approximate. When approximate succeeds, a description of the corresponding calculation is again placed on level arithm and the triggering strategy unit is updated. KS arithmetic executes the calculations specified in units on level arithm and writes the results on the other levels of the domain blacliboard. An update of units on level strategy by KS approximate triggers the KS update-solution, which ensures that the currently best solution found by the heuristic strategies, is represented in the problem description on level problem. Both print-result and print-result2 are triggered at the creation of a unit on level problem at the start of a problem-solving session, but are selected for execution only when the allotted time for the game has expired or when no other executable KSARs are available any more, respectively.

The here described implementation can be extended to improve the problem-solving capabilities of the system. Improvements are, e.g., addition of other heuristic strategies and rating of heuristic strategies according to expected applicability to a given problem (when the target number is large, while the other numbers are relatively small, a heuristic, multiplying two numbers before dividing the target number by the largest avajlable number to derive quotient and remainder, will succeed nore 
likely). These improvements can be added without having to change the present implementation, illustrating an important characteristic of the blackboard architecture, i.e., flexibility with respect to addition of KSs to a blackboard system.

\subsubsection{Control unit}

The control unit executes a loop, called the basic control loop. This loop is implemented as a succession of three procedures: Update-to-do-set, Choose-KSAR, and Interpret-KSAR (see also Figure 2.4 on page 21). These have more or less the same functionality as their namesakes in BB1 (Hayes-Roth, 1985). The following describes what each of the three procedures must take care of.

\section{Update-to-do-set}

- Keep track of the current cycle number: each time the three procedures Update-to-do-set, Choose-KSAR, and Interpret-KSAR have been executed and the basic control loop has executed one cycle, the cycle number is increased by one.

- Take the events occurred during the last completed cycle one by one and ask the ks-handler for the identifiers of all KSs with a matching trigger. The returned KS identifiers are given to the ksar-handler for creation of KSARs. The ksar-handler adds these KSARs to the list of triggered KSARs (stored on the control blackboard).

- Execute the precondition of all triggered KSARs. If the test of the precondition of a triggered KSAR has a positive result, that KSAR is moved from the list of triggered KSARs to the list of invocable KSARs. Then, the VALUES attribute in the KSAR can be updated.

- Compute the priority of all invocable KSARs. The procedure for this computation is domain dependent and has to be supplied by an application builder. Information that can be used for this includes all information on the blackiboard and information in the KSARs. 


\section{Choose-KSAR}

- Decide which KSAR should be executed next ${ }^{2}$ by using a procedure provided by the application builder. Choose-KSAR does not necessarily select the KSAR with the highest priority. A priority is calculated for each KSAR separately, while selecting a KSAR may involve aspects involving a number of KSARs as a group. Decoupling of individual priorities and selection permits the implementation of more sophisticated selection schemes.

- Test the precondition of the selected KSAR once more. Should this test fail, the KSAR is moved from the list of invocable KSARs to the list of triggered KSARs and the next best KSAR is taken as the selected KSAR and tested. If the test succeeds, the attribute VALUES of the KSAR may be updated in order to model the current state of the problem-solving process. The decision as to whether this is done actually lies with the application builder and is specified in the precondition. After a successful test ChooseKSAR continues.

- If so specified by the user, the user is asked at this point whether he or she agrees with the outcome of the selection process. If not, the user is invited to suggest another KSAR, which is then taken as the selected KSAR.

- Post the selected KSAR on the blackboard level chosen-action of the control blackboard panel.

\section{Interpret-KSAR}

- Execute the selected KSAR and change the status of this KSAR at the blackboard level chosen-action from chosen to executed or, if the solution is found, to solved. The procedure for determining when a solution is found has to be defined by the application builder.

${ }^{3}$ Actually, a KSAR is only a list of attribute-value pairs and cannot be executed itself. By executing a IKSAR we mean executing the corresponding KS instantiation, i.e., the KS, using the information represented in the KSAR, such as triggering-unit and values. 
The three procedures Update-to-do-set, Choose-KSAR, and Interpret$K S A R$ together form the main part of the module inference-engine, or inf-engine. The interface of the module inf-engine, however, consists of two procedures: one for initialisation and one for executing one cycle of the control loop.

\subsubsection{Interface}

A Blondie application is run from an interface. This interface, implemented as the module command-interpreter, or cmdint, allows a user to run the control loop and to view important data within the system. The functionality of the module cmdint can be summarised as follows:

- Initialisation of all the modules in Blondie.

- Starting the system by executing a special KS, provided by an application builder and used for specifying the problem to be solved (possibly via consultation of a user).

- Ordering the execution of a cycle of the basic control loop.

- Arranging communications between the system (Blondie plus application) and the user (application builder or user of an application). This task is done via the module trace. This module is discussed below.

- Provision of an exception handler.

Two modules remain to be described. These are the module trace and a. module for handling of input and output, called io-handler. In the module trace we collected a set of functions needed by a user of Blondie or of an application built in Blondie to be able to follow how the system proceeds. ${ }^{3}$

\footnotetext{
"It should be noted here that the module trace is equipped with functions mainly of use to an application builder. Explanation facilities suitable for an end user have to be implemented by an application buildex. Although there exist some examples in the literature of studies into generic facilities for explaining the behaviour of a blackboarl system (Hayes-Roth, 1984; Brunessaux \& Chevrier, 1990), these facilities are mainly useful to an application builder. There seem to be no obvious opportunities for defining generic, meaningful facilities that can explain the behavion of blackboard
} 


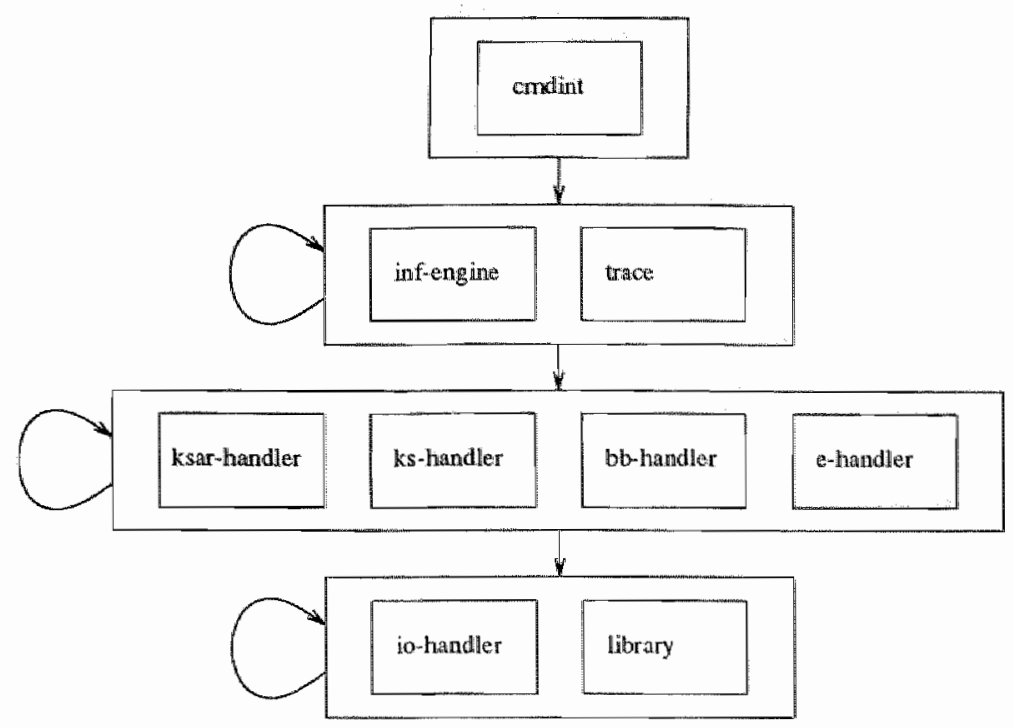

Figure 3.10: Module structure in Blondie.

The module trace provides the following functionality. Every $N$ cycles of the control loop (the user has to define the value of $N$ ) the user is invited to ask for information on the current state of the system. The user can ask the system to show specified units, blackboard levels, or blackboard panels, events, KSARs, the selected KSAR, KSARs triggered during the last cycle, etc.

Also, the user can ask the system to continue execution without halting. In that case, items from the possibilities enumerated above can be shown as well. Intermediate results and information obtained via the module trace can be printed on the screen as well as in one or nore prespecified files. The procedures for this are gromped together in the module iohandler.

systems to end rusers. In this respect the blackboard architecture behaves diferculy from rule-based expert systems, for which it has been clained regularly that they provide such opportunities in a natural way (e.g., Waterman, 1986, p. 28). "Hhs difference is due probably to the usual difference in granularity hetween rules and KSs, whle at the same time it is arguable whether the strugth of exphanation by explicity presenting a chain of executed rules provides moch insight 10 an average user of a rulle-based expert system. 
The modules in Blondie are shown in Figure 3.10. Not all interactions between the modules are shown, as they can become quite entangled. In general, the hierarchy of the modules is such that the procedure calls are from a module in one group to modules in the same or lower groups as suggested by the arrows.

\subsection{Evaluation}

After Blondie was written and some preliminary testing had been done we implemented two small applications for final testing of Blondie and for partially evaluating the blackboard architecture. These test applications were the number game and a system for finding a path connecting two points in a graph. This later test application has been used also in testing and debugging Blondie's successors, Blondie-II and Blondie-IIII.

The blackboard system for solving the number game was compared with an implementation in Pascal for the same problem, which used brute computational force to calculate all possible combinations. When five or six of the given numbers must be combined to approximate the target number closely, the implementation in Pascal is not able to find this solution within the time limit set for this game due to combinatorial explosion. This suggests the need for heuristic problem-solving strategies. Although these test applications were indeed simple, we did arrive at some initial conclusions, both with respect to Blondie and to the blackboard architecture in general. These conclusions are discussed in this section.

\section{Flexibility}

Before we had entered the design phase of Blondie, we expected that blackboard systems would be flexible. By this we mean actually three things. Firstly, we expected that blackboard systems would be flexible in the employment of strategies for obtaining solutions, i.e., they can change the strategy according to changing circumstances. Secondly, it was suggested in the literature that implemented control strategies can be changed quickly and easily by an application builder, due to the fact that control aspects are made explicit. Finally, the blackboard 
architecture promised flexibility with respect to the use of $\mathrm{KS}$, allowing easy addition, replacement, or removal of $\mathrm{KSs}$.

Based on our initial experiences, we cannot say much about the first sense of flexibility, as our solution to the test applications were too straightforward and did not need flexible problem-solving strategies.

About the second and third sense, we can be more specific. It was confirmed that Blondie is flexible with respect to those two aspects. Problem-solwing strategies as well as the set of $\mathrm{KSs}$ can be changed fairly easily. We started the implementation of the number game using a subdivision of the problem-solving process which resulted in too many KSs. In a blackboard system with such a fine-grain subdivision in KSs the time spent in executing the control loop can exceed the time spent executing $\mathrm{KSs}$ by far, as happened in our initial implementation. By changing the granularity and adapting the attributes in the KS descriptors used for calculating priorities of KSARs, we were able to modify the initial implementation rapidly towards a system with an acceptable performance.

Building an application in Blondie requires knowledge about how to program in the programming language Pop-11. Apart from implementing KS descriptors and action parts, an application builder must supply procedures for calculating priorities of KSARs, for selecting KSARs, and for checking whether a stop criterion is met.

\section{Speed of application development}

One of the advantages of using expert-system shells or blackboard shells is the ease and speed with which applications can be developed, as generic components of the architecture are readily available. This seems to be true for Blondie as well. It took about six man days to produce the first wersion of our game-playing program (which included some debugging of Blondie as well). After that, the flexibility of the blackboard architecture aided in ons prototyping efforts in finding a suitable implementation. The availability of a blackboard shell allows an application builder to concentrate almost exclusively on how to solve a problem.

At first glance a comparison between the time it took to implement the game in Blondie and the time needed for implementing the straightforward Pascal program works out unfavourably for Blondie. This Pascal 
program consisted of only 175 lines and was written in two hours. However, we must stress that this comparison is not a fair one. Calculating all possibilities leads to a very simple program. We may assume safely that implementing heuristics in the Pascal program as used in Blondie and prototyping the system until sufficient performance is obtained would need a considerable amount of effort and lines of code. As prototyping is often needed when a suitable problem-solving strategy is not known beforehand, this last comparison is the one which actually should be used when discussing the merits of a blackboard shell.

However, we found that if systems such as Blondie are to be used for serious applications, some additional tools would be useful, if not indispensable. We want to distinguish between two categories of tools necessary for implementing an application in the shell Blondie. One category consists of tools assisting in the syntactically correct definition of the blackboard panels, levels, unit attributes, and the interfaces of the KSs. These tasks could be done by a syntax checker; a relatively simple, but useful aid. In fact, we implemented such a tool for Blondie as a result of our initial experience.

The other category is formed by tools for implementing the action parts of KSs. The action part of a KS in Blondie must be a Pop-11. procedure, but it can make use of Lisp or Prolog code, a rule-based system or a frame-based system, etc.. As long as the action part of a KS consists of Pop-11, Prolog, or Lisp code, it is hard to think of useful tools for developing this action part. If, however, the action part is to be for instance a rule-based or a frame-based system or any combination of these two, shells for implementing these action parts should be available together with tools supporting the addition of domain knowledge.

\section{Evaluation of some of our design choices}

We evaluate some of the choices made during the design of Blondie. This evaluation is based on the initial experiences with Blondie. A bet. ter understanding of the impact of some design options was achieved later, i.e., after experimenting with applications written in Blondie-II and Blondie-III. Chapters 6 and 7 try and capture this better understanding and provide a more thorough discussion of design options and their consequences. 
In our opinion, the ability to divide the blackboard into any number of panels is a useful method for restricting the number of blackboard levels on each panel, thus increasing the surveyability of the blackboard during the application-development process. An extension of this idea is represented by the incorporation of object-oriented modelling techniques for subdividing the blackboard using class hierarchies, as in, e.g., Hearsay-III (Erman et al., 1981), and Poligon (Rice \& Aiello, 1989).

The triggering mechanism for $\mathrm{KSs}$ did not cause any problems during our initial use of Blondie (a KS can trigger only on the occurrence of one event and not on combinations of events, e.g., after the occurrence of both event $A$ and event $B$ ). In later applications it was felt that allowing specification of triggers to match with combinations of events, using AND, OR, and FOLLOWED-BY constructions, would be a useful improvement. In Blondie-III such a mechanism was eventually implemented (see Chapter 5).

A problem concerning events is that too many are created. An illustration of this is provided by the following. On almost every cycle of the control loop, the system creates triggered KSARs and changes the status of KSARs from triggered to invocable. This produces many events, as lists of triggered and invocable KSARs are stored on the control blackboard panel and each modification of a unit results in an event. These events, however, are used rarely and unnecessarily restrict the efficiency of a blackboard application in Blondie (all events are matched against the triggers of all KSs).

A solution to this problem would be to include a mechanism creating an event only when such an event would be meaningful. This could be implemented by adding procedures to the blackboard-handler allowing modification of the blackboard without generating events. Conceptually, however, such an approach violates the inclependence of $\mathrm{KSs}$; during the implementation of one KS it becomes necessary to have a clear idea about which interactions with the blackboard will be of importance to other KSs and which will not. We feel that an important principle of the blackboard architecture is that KSs can be seen as black boxes which can be developed almost completely independently.

An additional solution would be to record for each KS the events it is interested in and remove, or not generate, all events that no KSs are interested in. The triggering mechanism as implemented for Blondie-III 
uses such recording of relevant events.

In the blackboard system HASP ( $\mathrm{Nii}$ et al, 1988 ) events are classified, and the system takes care of only the events that are from the right class. A separate blackboard level (typically the level 'focus') can be used to record which classes are currently relevant.

\section{Efficiency}

Some remarks about the efficiency of Blondie are in order. When we started the design of Blondie we did not strive for an efficient system. One of our aims was that Blondie should be modifiable and extensible. So, all data structures are lists (even the blackboards), meaning that they can be adapted easily. The penalty for this is inefficiency. Much time is spent during pattern matching and garbage collection (approximately twenty-five percent).

Better efficiency could have been achieved by implementing data structures via, e.g., arrays. Or, a relational database could have been used to implement the blackboards (as was done in Hearsay-III), thus improving the efficiency of the pattern matching operations for searching for specific data on the blackboard.

Perhaps this is the right place to point out another feature of relational databases, which might prove to be useful for implementing a blackboard. Relational databases normally provide standard routines for coping with concurrent access of data stored in the database. This feature can be used in parallel blackboard architectures. As we describe in the next chapter, concurrent access of the blackboard by concurrently operating $\mathrm{KS}$ s is an issue which needs to be addressed and dealt with in parallel blackboard systems.

The granularity of KSs plays a role in the efficiency of the system. Initially, in our number-game test application we used a large number of small KSs. As we found out soon, this was not a good jdea. We then transformed the system to one using a smaller number of larger $\mathrm{KS}$. Then, the time spent during execution of the control loop became sufficiently better in proportion to the time spent executing the KSs to be able to meet the time constraints of the game. At first, approximately ninety percent of the time was spent during the execution of the control 
loop and ten percent during the execution of KSs. After having restructured the KSs, the time spent during execution of the control loop was sixty to eighty percent of the overall execution time, depending on the total amount of time spent for the complete problem-solving process (due to fixed start up and completion costs).

Although the program became fast enough, the warning is clear. Often, it is not possible to avoid extensive computations in the control cycle of a blackboard system. Yet, time spent at the control level can lead to time saved at the domain level. Of course, a portion of the costs of the control unit of Blondie is just overhead and not actually part of the control task. This is the reason why a blackboard architecture may be suited in some cases only for prototyping, after which a much more efficient system could be built while using the insight gained by using the blackboard architecture. 


\section{Chapter 4}

\section{A Parallel Blackboard Framework for Robot Control}

After we had implemented and evaluated Blondie, the robotics research group was developing a robot cell to test theoretical ideas in a practical setting. One of the research topics in this robotics programme was the development of a supervising system for a robot cell. We found that the requirements for such a supervisor, as formulated by the research group, showed interesting similarities with characteristics of the blackboard architecture. We decided to use Blondie for building a robotcell supervising system. However, concurrent operation of components within a robot cell could not be handled by the design of Blondie. In order to include such a facility, we developed a new blackboard framework, based on Blondie. The resulting parallel blackboard framework, called Blondie-II, uses time sharing to simulate concurrent execution of know]edge sources. We did not choose to implement a real, parallel robot-cell supervisor as we were interested primarily in studying the feasibility of the idea of using a parallel blackboard architecture for robot-cell control.

This chapter first discusses some aspects of the domain of robot cell control and illustrates how requirements for a robot-cell supervisor matched with characteristics of the blackboard architecture. Section 4.2 gives a description of Blondie-II, mainly by addressing the modifications with 
respect to the sequential blackboard shell Blondie. Next, Section 4.3 discusses some related work. The chapter concludes with an evaluation of Blondie-II and applicability of the blackboard architecture to the domain of robot control.

Material from this chapter has been published previously as a report (in Dutch) in (Velthuijsen, 1987a). A manual for Blondie-II (Velthuijsen, 1987 d) provides additional information. Several papers on Blondie-II have been published as well (Velthuijsen et al., 1987a; Velthuijsen et al., $1987 \mathrm{~b}$; Velthuijsen, 1987e).

\subsection{The application — robot control}

More often than not, a robot executes its tasks in continuous interaction with its environment. Usually, the environment for a robot consists of a number of different devices which together with the robot perform a. given task. These devices may include, among others: visual and tactile sensors, separate processors for executing computational tasks, such as the calculation of collision free paths; operators which import objects into or export them out of the environment; and the actual robot arm and robot hand. The environment of a robot even may include other robots. The robot and all the devices in the environment performing a given task are together called a robot cell.

Figure 4.1 depicts the experimental robot cell arranged by the robotics research group. The cell was developed for mail package handling. Mail packages enter the robot cell under a vision component. This vision component is used to determine size and orientation of each entering package. Then, packages are put aside on a buffer until the robot can place them in a container. Two restrictions apply for the placement of packages in a container. Firstly, packages must be placed in such a way that the stacks will not tumble. Secondly, the fraction of the total volume in a container eventually occupied by packages must be maximised. Algorithms were developed to ensure placement of packages present on the buffer according to these constraints. Apart from these algorithms, another algorithm was used to calculate collision free paths for the robot and robot arm. The robot cell supervisor is used to integrate the diverse components in this robot cell to ensure correct interchange between all the components and to control the execution of algorithms at opportune 


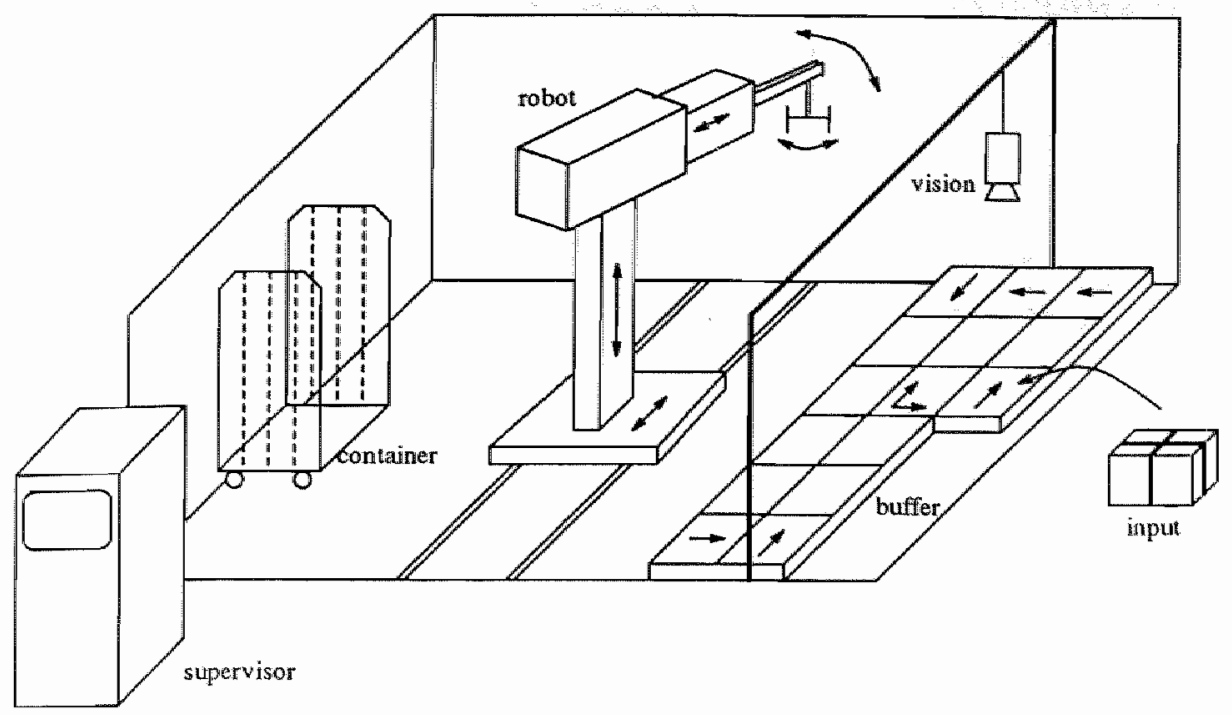

Figure 4.1: The experimental robot cell at PTT Research.

moments with the appropriate input data. The following requirements were formulated for such a supervisor:

- To start and control components and subprocesses within the robot cell. The supervisor has to be able to determine which component should perform which activity according to the current situation within the robot cell and the overall task at hand. Control of subprocesses also includes halting subprocesses in cases of emergency, and decisions to allow only a limited amount of time for a subprocess to complete in cases of urgency.

- The supervisor has to be able to deal with diverse components with possibly different internal representations. The realisation of such incorporation of diverse components includes the provision of a unified method for communication. An additional requirement in this respect stems from the idea that a robot cell might itself be part of a larger automated environment. This means that the robot cell supervisor must be able to receive messages and commands from outside the robot cell and to incorporate these communications. 
- The supervisor must be able to ensure consistency of data to avoid the possibly damaging effects of inconsistencies within the supervisor.

- The supervisor must be able to handle exceptions, faults, and nearfaults as much as possible without halting the complete robot cell: the supervisor must react to all kinds of situations in a flexible way.

- The supervisor should be able to detect decreasing workload and to ensure that an increase of avalable time is used for optimising subprocesses, such as algorithms for calculating placement of packages in the container.

- The way the supervisor reacts to situations in the robot cell must be easily modifiable in order to experiment with different strategies for executing the tasks of the robot cell.

- It must be possible to add, remove, and exchange components without rebuilding the robot cell supervisor. While the robot cell is under development, it should be possible to experiment with different versions of the same component or with different combinations of components.

- To ensure efficient use of the components and to meet time constraints, it is necessary that these components can execute appointed activities concurrenty. The supervisor has to support this.

The role of the supervisor in a robot cell can be illustrated with the description of the following architecture, also shown in Figure 4.2 (Bakker. 1986). The heranchical and modular structure of this architecture provides some of the Hexibility needed for adding, removing, or exchanging components within the overall system. The machines in this illustration can be actual, physical machines as well as computer programs, or algorithms, acting as virtual machines. The machines are controlled directy by drivers. The supervisor communicates with the drivers via machine specialists. The machine specialists act as 'translators' between the supervisor and the corresponding driver, which may reside on a different processor. All commmnication between machines is via the supervisor. 


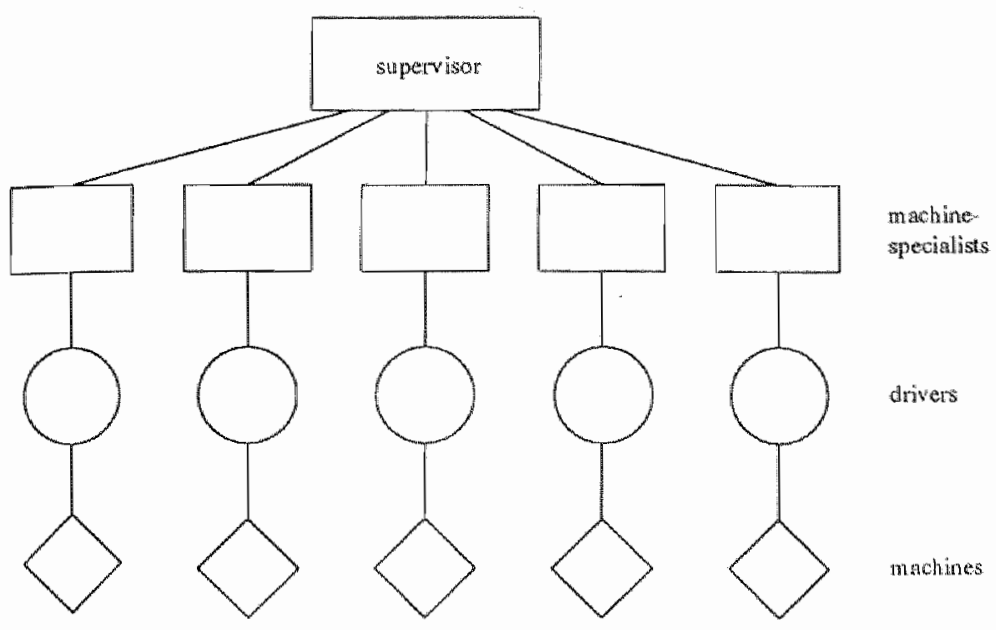

Figure 4.2: A 'flexible' robot cell architecture.

Mainly because of the need to react to situations as they occur, even to faults, as described above, attention was directed towards the devel. opment of an 'intelligent', knowledge-based supervisor, using artificialintelligence techniques rather than a programmed, procedural approach. Robot planning, as exemplified by the two planning systems STRIPS (Filies \& Nilsson, 1971) and ABSTRIPS (Sacerdoti, 1974), was not considered suitable, due to its focus on goal-directed reasoning. Disadvantages of a goal-directed approach are that it seens to be less robust in the event of unforeseen situations and that it is better suited for achieving one goal at a time, instead of continuously achieving a set of goals, as is the case in an ongoing robot-cell control application. In contrast, we were more interested in developing a data-driven or event-driven stpervisor and thus found that rule-based programming or a blackboard architecture might be worth examining in this context.

Some additional inspiration was fonnd in (Kali et al, 1986) presenting a knowledge-based robot cell supervision system. The architecture proposed there is shown in Figure 4.3. The subsystem in this architecture actually responsible for making decisions integrates information from its sensory subsystem and its motion controller, together with "knowledge" from a separate knowledge base and information about the current state 


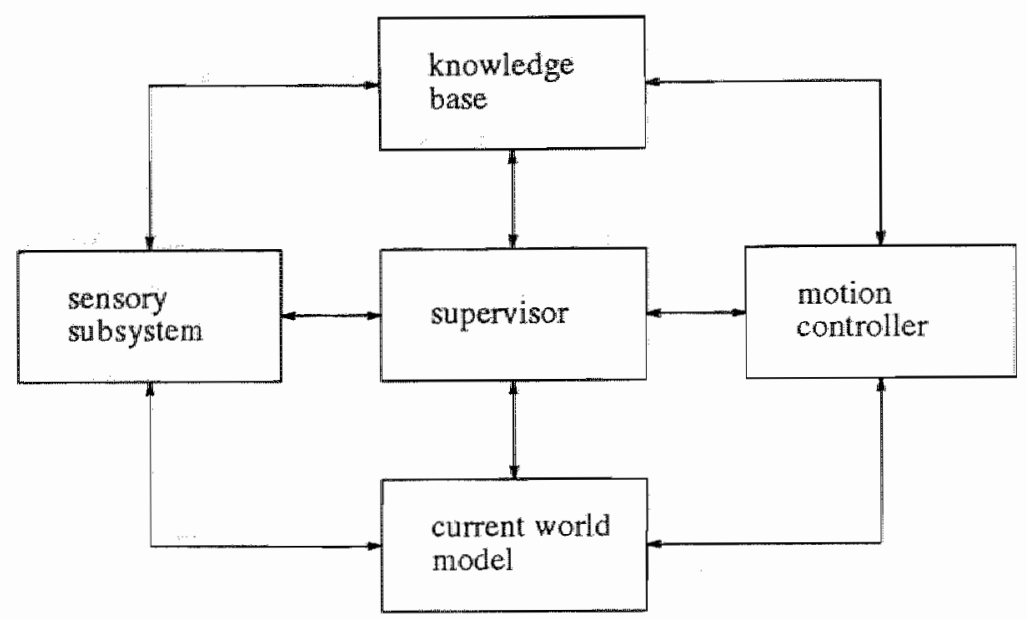

Figure 4.3: Supervisor interaction as proposed in (Kalk et al, 1986).

of the robot cell environment stored in the current world model. The sensory subsystem and motion controller can each be thought as consisting of one or several of the machines and drivers as shown in Figure 4.2.

We found that the architectures as shown in Figures 4.2 and 4.3 can be transferred easily into a blackboard architecture. We propose the following mapping for robot-control applications built with Blondlie-II. A knowledge source can be used to represent a machine specialist or even a machine specialist together with corresponding driver and machine. Knowledge sources can be used also to implement the knowlerge base. The robot-cell supervising task can be mapped onto the control unit of a blackboard system together with a collection of dedicated control knowledge sources. Communication between the components may occur via the blackboard, whilst the blackboard also affords an opportunity for representing the current world model. The requirements mentioned above can then be met by this blackboard architecture, provided a version is implemented allowing concurrent execution of knowledge sources. After all, the blackboard architecture provides flexible control and allows components to be exchanged comparatively easily. Furthermore, the blackboard architecture provides a means for integrating different components. The blackboard architecture also provides opportumistic 
problem solving, which makes it possible to select new tasks; based on information on the current state of the problem-solving process.

As stated at the beginning of this chapter, we implemented the parallel blackboard framework Blondie-II as a tool for research and prototyping, in order to be able to investigate concurrent blackboard architectures for use in robot control, and to explore their properties and suitability for this task. During this investigation we wanted to address the following questions.

- Which subprocesses should be executed in which order and which subprocesses should be executed in parallel in order to meet timeconstraints. This should be addressed under varying degrees of worliload.

- How can a flexible method of assigning priorities be used to ensure optimisations in case of decreasing workloads?

- What information must be exchanged, and when, between components in the robot cell?

- How can errors be detected and handled within the system? At the beginning of the development of Blondie-II, it was felt that the event-driven nature of blackboard architectures would be helpful in detecting and reacting to exceptions and faults. Validating this would be one of the major goals of using Blondie-II in experiments.

\subsection{Parallelisation of Blondie}

The blackboard metaphor describes a situation where several 'specialists' cooperatively solve a problem by communicating via a blackboard. This metaphor is inherently concurrent: each "specialist" is a "processor" capable of executing his or her self-appointed activities, based on opportunities as they present themselves on the blackboard. The relative independence of these activities suggests concurrent execution. This oflers an opportunity to speed up the problem-solving process or, in a robot-cell control application, to make more efficient use of avalable components and processing power. For, when a task uses different devices for performing (sub)tasks, segnential scheduling of independent 
(sub)tasks seems at least strange and unnecessarily inefficient. ${ }^{1}$ Thus, the idea of a parallel blackboard architecture is a natural one.

There are, of course, different approaches to developing a concurrent blackboard architecture, the most basic distinction being between a parallel blackboard architecture and a distributed one (see also Section 2.4). As one of the requirements for our robot-cell supervisor was expressed as the need for consistent data, we opted for the parallel blackboard architecture approach which makes it easier to ensure data integrity. A parallel blackboard architecture can, basically, be implemented in one of two different ways, basically. The first is closest to the blackboard metaphor and consists of a set of knowledge sources (KSs), each running on a separate processor, executing a $K S$ cycle, i.e., alternately monitoring the blackboard for interesting information and executing some activity upon detection of such information. In such an architecture the processors integrated in the overall system are typically dedicated to the execution of one kind of activity. The second way can best be seen as an adaptation of the sequential blackboard architecture with a control cycle. Here a control unit decides which activities should be executed and distributes these activities over available processors. We opted for this second version, as it seemed to provide the more natural extension of Blondie. Moreover, we wanted to design a tool for experimenting with different robot-cell organisations and varying distributions of activities over processors. Such a tool is supported better by the second version.

We stress here that the granularity of parallelisation in Blondie-II is at the level of KSs instead of, e.g., the level of rules within KSs. We were interested in creating an environment for concurrent execution of recognizable tasks within a robot cell, rather than to facilitate concurrency of more fine-grain activities. An example of a robot-control system using parallel evaluation and execution of production rules is presented in (Heril \& Henseler, 1986; Herik et al., 1988). In general, fine-grain parallelisation presents more synchronisation problems than coarse-grain parallelism, hence our preference for the selected level of concurrency.

Several changes had to be made to the sequential blackboard framework Blondie to enable the parallel execution of subtasks (KSs). The control mechanism had to be modified in order to be able to execute KSs con-

\footnotetext{
This does not necessarily mean that there are always useful (sub) tasks to perform for each component.
} 
currently, while also the selection and scheduling of the KSs had to be adjusted.

Another major modification concerns blackboard access. The blackboard handler must now be able to support simultaneous blackboard interaction by the KSs. Moreover, access of a single blackboard by concurrently operating KSs might result in loss of data integrity on the blackboard. Violation of data integrity occurs, for instance, when one KS reads information from the blackboard, processes this information, and subsequently tries to modify the blackboard, assuming that the original information has not yet been altered, although, meanwhile, it has been changed by another KS.

One of the often advocated features of the blackboard architecture is the ability to interpret uncertain or conflicting hypotheses on the blackboard. The usefulness of this feature has been noted especially in distributed blackboard systems, where several complete blackboard systems cooperatively solve a common problem (Erman et al., 1980; Lesser \& Corkill, 1981).

This feature, however, is mostly useful in applications where KSs act only as perceivers: components which only interpret data, but do not alter their environment. The ability to interpret uncertain and conflicting hypotheses does not suffice in applications such as robot-cell control, where KSs can operate as effectors: components which may alter their environment. There, information on the blackboard can represent. objects in the real world. Unreliable or incorrect information on the whereabouts of moving objects could result easily in physical damage to one of these objects. A parallel blackboard shell for robot control, therefore, must support procedures for preserving consistency of data on the blackboard.

The following sections address, in turn, the necessary modifications of the original Blondie architecture as mentioned above. First, we describe the general organisation of Blondie-II. Then, in Section 4.2.2, we describe how communication between components in Blondie-II is implemented. Section 4.2 .3 presents modifications of the control unit to support parallel execution of KSs. Finally, Section 4.2 .4 addresses modifications to the blackboard handler. 


\subsubsection{Physical and virtual processors}

Parallelism is simulated in Blondie-II via time sharing. Processes are used to represent real-world devices or processors. In our design we distinguished between virtual processors and physical processors. Physical processors correspond to the processors or devices as they would exist in a real-world application, whereas virtual processors correspond to processes which can be recognised in the system. It is conceivable that several virtual processors will be run on the same physical processor. In that case the virtual processors run, at least conceptually (e.g., via time sharing), in parallel on that physical processor.

In a robot-cell control application, e.g., one could implement the control unit and processes for the execution of an algorithm for calculating collision-free paths on one physical processor, but also decide to use different physical processors when the two activities combined use too much processing power for a single physical processor. Blondie-II distinguishes the following different virtual processors:

- One virtual processor for the control unit.

- One virtual processor contains the blackboard.

- At least one, but usually more, for executing $K S s$.

Virtual processors are implemented as processes in Blondie-II, therefore the terms denote the same hereafter. The control unit and the blackboard are represented by separate processes, as they need to be available for interaction with concurrently operating KSs at all times. The control unit has to be able to select a new task whenever a KS process (a. virtual processor dedicated to executing $\mathrm{KS}$ ) is ready, while the blackboard process has to be available for interaction with the KS processes. The process containing the control unit is called control process.

Implementing (a simulation of) an application in Blondie-II includes finding an optimal distribution of virtual processors over physical processors. Figures 4.4 and 4.5 illustrate two possible distributions.

For a given run of the system this distribution of virtual processors over physical processors is fixed. Thus, it is known before a certain configuration is run on which physical processor a virtual processor can be 


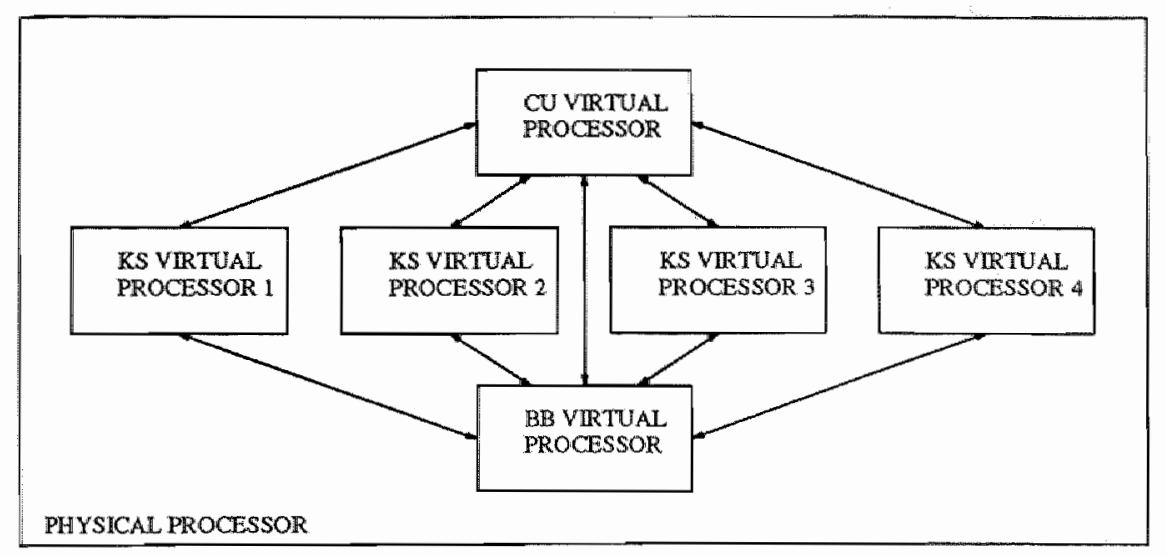

Figure 4.4: All virtual processors on one physical processor.

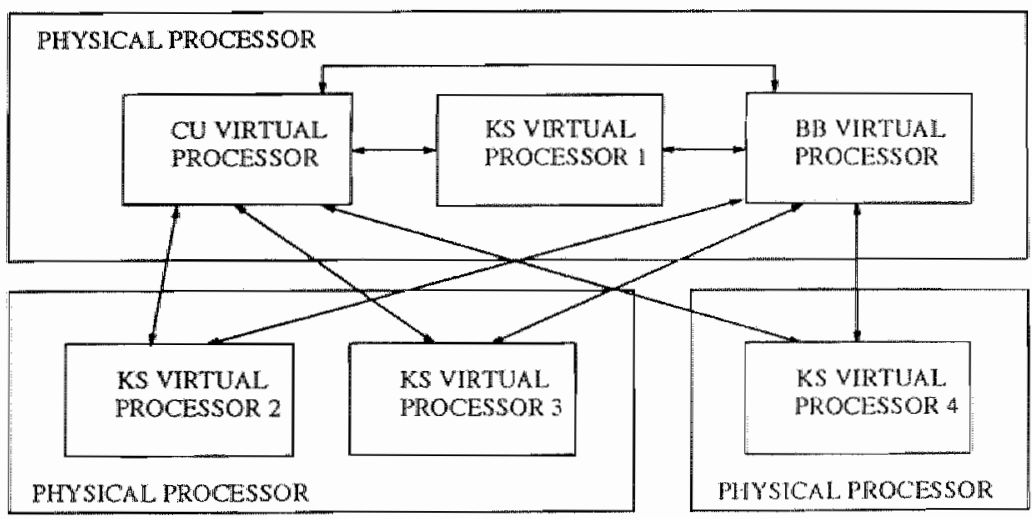

Figure 4.5: Virtual processors distributed over three physical processors 
found. For each $\mathrm{KS}$, the physical processors which can execute it must be specified. This is done by specifying the processors which can execute the KS in the KS's descriptor. This way a mapping is created from KSs to physical processors. This mapping is shown in Figure 4.6. The mapping is a composition of two other mappings. The first of these is a mapping between KSs and virtual processors and is defined in the KS descriptors. The second of these maps virtual processors onto physical processors, as defined by the distribution of virtual processors over physical processors. This mapping need not be one-to-one, one-to-many, or many-to-one. The following illustrates this. One can imagine an application where a specific physical processor can execute only one $K S$ (e.g., a robot arm only equipped to move from one place to another), while other physical processors can execute a number of different KSS (e.g., a numerical processor for executing different algorithms, such as a collision-free path algorithm and loading algorithm). Analogously, some KSs can be executed by only one physical processor (again, e.g., the move of the robot arm from one place to another) and others can be executed by more than one physical processor (e.g., when several numerical processors are available for executing algorithms). Our architecture allows for all these options, clue to the specification of suitable virtual processors in the KS descriptors and to the distinction between physical and virtual processors. The architecture is also flexible enough to change easily between different distributions as is essential for an experimentation environment.

\subsubsection{Communication}

Processes in Blondie-II need to communicate with each other. We use remote procedure calls, which are implemented using message passing facilities of the UNIX System $V$ operating system ${ }^{2}$. Eacl process has associated with it its own message queue, where messages are kept un. til that process can read the contents. Implementation details are described in (Velthuijsen, 1987b). We note that this information exchange via messages does not provide another communication medium than is already provided by the blackboard: message passing makes interaction with the backboard possible. The procedures handling messages are

${ }^{2}$ UNIK is a rademark of AT\& T Bell Laboratories. 

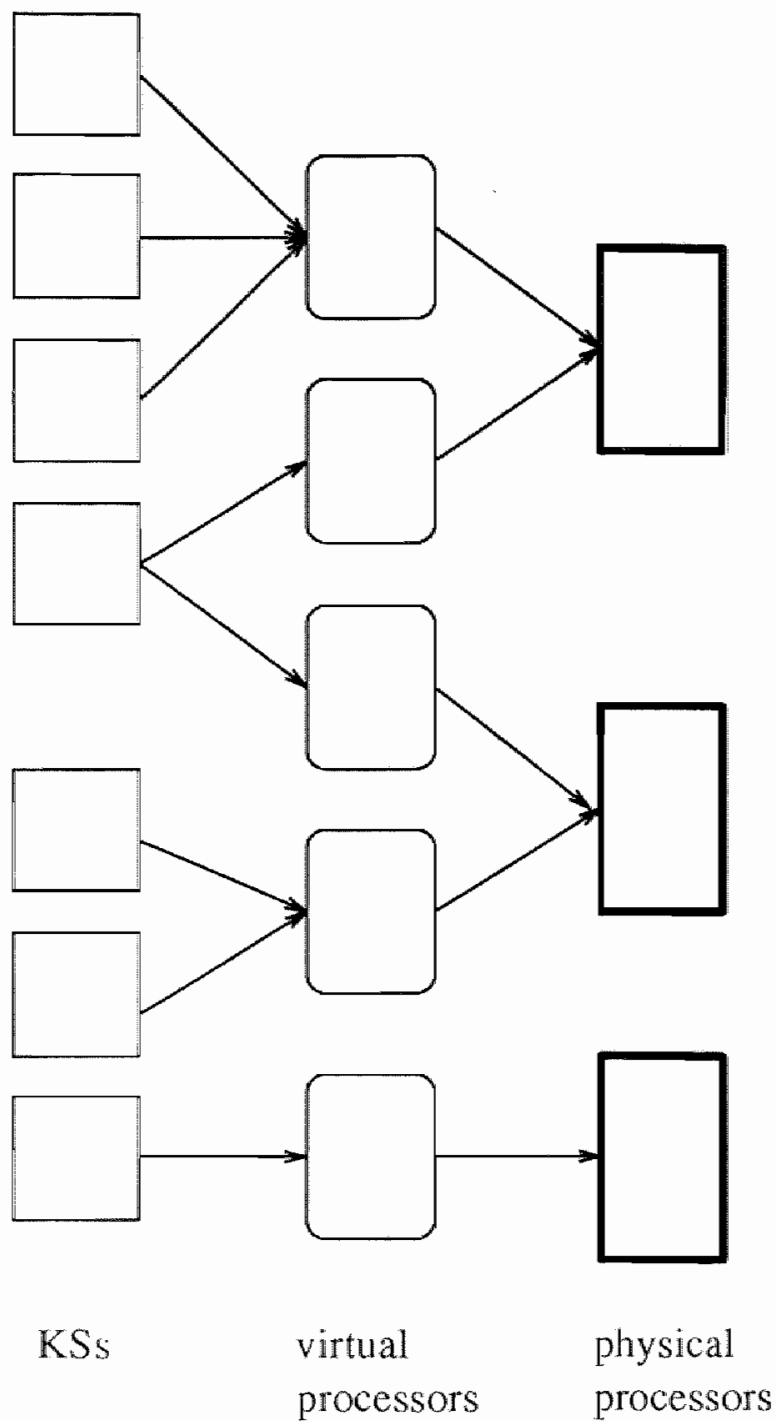

Figure 4.6: Mapping between KSs and physical processors. 
grouped together in the new module msg-handler. We recognise the following exchange of information in a Blondie-II application (Figure 4.7 illustrates the communication channels in Blondie-II).

- Communication with the blackboard. Both control unit and KS processes need to inspect data on the blackboard or to modify data on the blackboard. We use different message priorities to distinguish between requests. Requests from the control process have a higher priority than requests from KS processes. Requests from KS processes can have one of two different priorities: high or low. This is done to enable almost immediate processing of urgent messages, concerned, e.g., with exception handling.

- Communication between the control process and KS processes. This communication is used only by the control process to order KS processes to perform a certain activity and by a KS process to report that an activity has been completed. A command to perform a certain activity contains the information of a specific KSAR. The KS process determines from this KSAR the action part of the $\mathbb{K S}$ to be executed and the specific data to use it on.

- Communication for exception handling. Whenever an error or exception is observed, appropriate steps have to be taken. We can distinguish between urgent errors and milder exceptions. Information about urgent errors can be broadcast via an interrupt mechanism. This mechanism is reserved for those situations where an error could mean instant damage. Milder exceptions are handled through 'normal' channels. A message, containing information about a specific exception, can be sent to the blackboard handler (using high priority), thus triggering appropriate KSs for determining recovery procedures.

In order to ensure immediate execution of KSs triggered specifically for recovery purposes, one has to fledicate virtual processors for this task; otherwise all processors might be busy doing other things. The distinction between physical and virtual processors makes it possible to have virtual processors available for the execution of recovery KSs at all times without actually losing processing power of physical processors. An example of this is a system where on one physical processor 


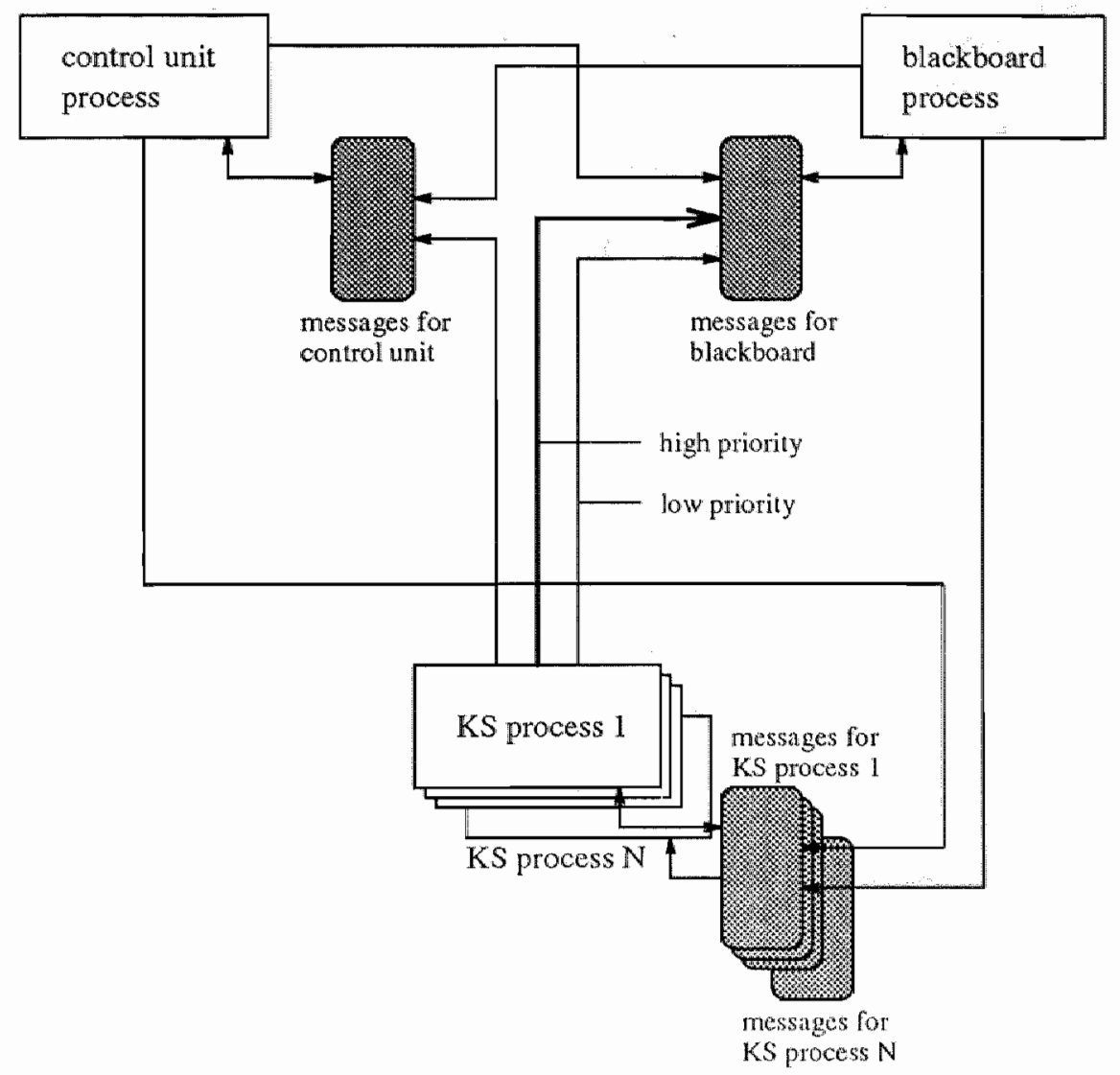

Figure 4.7: Communication in Blondie-II. 
two virtual processors are defined and implemented as two processes operating via time sharing. One of the virtual processors executes 'normal' KSs, whereas the second virtual processor is kept in reserve for exception handling. As long as the second processor is idle almost all processing power of the physical processor is dedicated to the first virtual processor, but as soon as the second virtual processor receives a task, both virtual processors are given a share of the processing power of the physical processor.

It is this mechanism which avoids the need for interrupting KSs in cases of non-fatal. errors. Interruption of KSs is undesirable as it necessitates extensive administrative activities for ensuring consistency of data related to the interrupted KSs. This involves data on the blackboard accessed by the $\mathrm{KS}$ as well as data in the control unit representing the assumption that corresponding KSARs are being executed or have been executed.

\subsubsection{Modifications of the control unit}

The control processor administers of the processors in the environment, or, in this simulation version, of the virtual processors, implemented as processes. In this scheme, for each virtual processor, the KSAR being executed is indicated, or the processor is marked as idle. As mentioned above, the KS descriptors contain an indication of the processes able to execute that KS. This information is also made available in the KSARs. Figure 4.8 presents an example IKS descriptor for Blondie-1I.

The main modification of the control unit necessary for parallel KS execution, is to substitute Interpret-KSAR of the basic control loop (the execution of a selected KSAR, see Chapter 3) by a command to another process to execute the chosen KS. The new control loop is shown in Figure 4.9. When a KS process is ready to execute a KSAR, it semls a message to the control process to announce that it is free to accept another KSAR. For all KS processes these messages are received and kept in a single list within the control unit. The control unit takes the messages from the list and thus determines for which KS processes a now task should be selected. Then, the control unit updates the sets of triggered and invocable KSARs using the events that occurred since the last updating took place together with the current state of the black- 


\begin{tabular}{|lll|}
\hline NAME & move-arm & \\
DESCRIPTION & Move the robot arm & \\
TRIGGER & KIND & new \\
& BLACKBOARD-LEVEL & robot-task \\
PREGONDITION & is-arm-at-begin-point & \\
PROCESSOR & robot-arm & \\
VARLABLES & begin-point, end-point & \\
FEATURES & APPROX-DURATION & 1.2 \\
& RELIABLLTY & 1 \\
& NEEDS-LEVELS & collision-free-path \\
& READ-LOCK & robot-tast \\
& collision-free-path \\
& WRITE-LOCK & package-position \\
\hline
\end{tabular}

Figure 4.8: Example of a. $K S$ descriptor in Blondie- II. The features were tailored for the robot cell control application. The use or read locks and write locks is explained in Section 4.2 .4 .

board. Events can be made available to the control process by a call to the blackboard process to return a list of all events that occurred since the last such call. Next, the control unit checks which invocable KSARs cam be executed by the available processes. For these KSARs a priority is computed.

An application builder must specify (via a Pop-11 procedure) how prionities are calculated and (via another procedure) how a KSAR is selected eventually for execution. It is imaginable that at one moment there do exist KSARs, executable by a free KS process, all with a very low priority. Hexecuting one of these low-priority KSARs makes it impossible to execute a ISSAR with a higher prority immediately, when such a KSAR would be created during the execution of a low-priority lsSAR. Dividing the selection tasti into a priority calculating procedure and a separate selection procedure enables the use of thresholds, thus avoiding the execution of rather pointless activities just because no other, more useful, activities can be detected at the time.

When a KSAR is selected, a command is sent to an appropriate KS process to execute the chosen KSAR. After this activation, the control unit does not wat for the termination of the execution of that hSAR. 


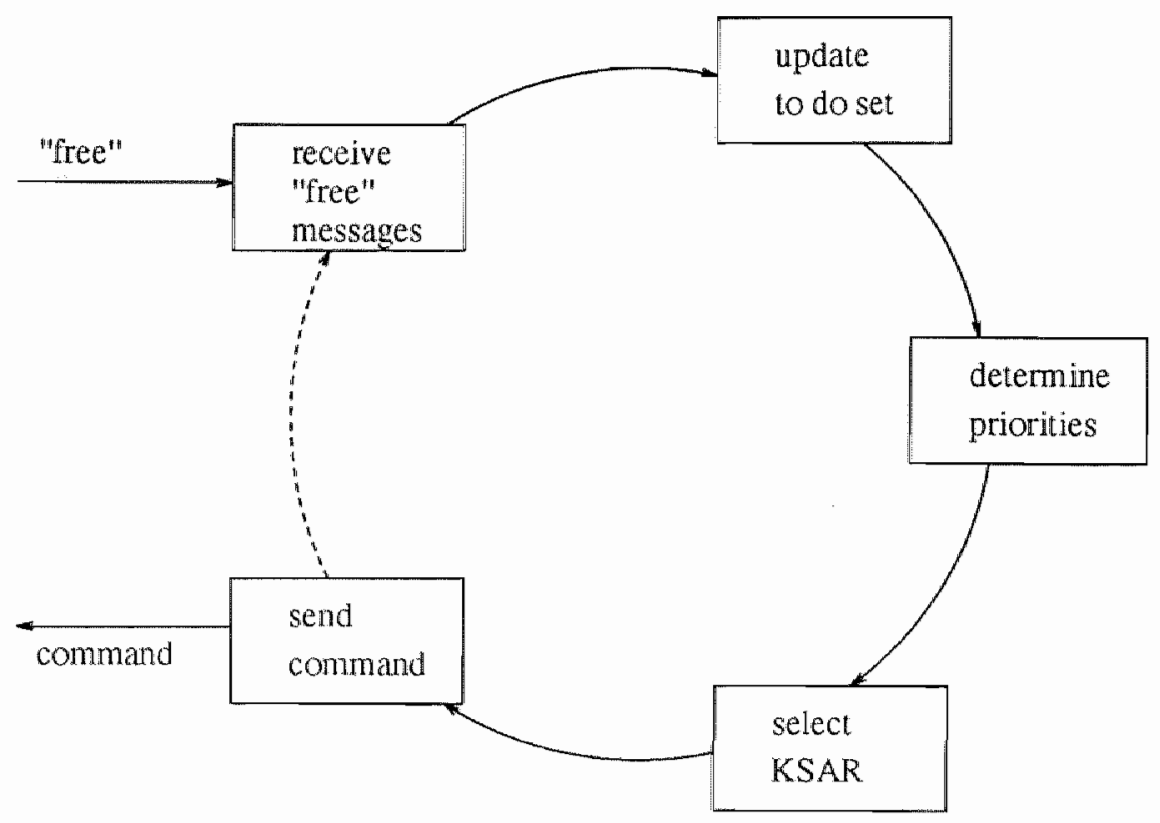

Figure 4.9: Control loop for parallel ISS execution. 
but continues immediately with the selection of a task for the then idle KS processes. If no invocable KSAR is found for these processes, the control unit waits for other processes to become idle.

The control unit in Blondie-II still performs a loop. However, now the control loop can be in an idle state. One example of an idle state occurs when no KS processes are free. Then the control process is polling its message queue regularly to wait for messages from the KS processes, indicating that a $\mathrm{KS}$ process has become available or that an exception has occurred. Another idle situation may arise, when there are free KS processes, but no invocable KSARs that can be executed by any of these KS processes. This idle state terminates, when either another KS process becomes free, or when a new call to the blackboard process for newly created events leads to the creation of KSARs that can be executed by any of the currently free KS processes. As in most other blackboard architectures, it is also possible that the control loop gets into an idle state when no invocable KSARs are available, while no KS is being executed either. In that case the problem-solving process halts. Blondie-II detects such a situation and notifies the user.

KS processes themselves execute a cycle. This cycle alternately executes a. KSAR and waits for a message specifying a new KSAR for execution. The module ks-handler in Blondie-II is divided conceptually into two parts. One section resides in the control unit and basically emulates the behaviour of the ks-handler as provided in the sequential system Blondie, while sending requests for execution to the second part. The second part resides in the KS processes and talies care of the actual work.

\subsubsection{Modifications of the blackboard handler}

The bb-hander in Blondie-II, similar to the ks-handler, is split over different processes and implements remote procedure calls. The control process and the KS processes contain procedures which, when called, transfer the execution of the called procedure to the blackboard process by sending and receiving messages. The blackboard process performs a loop in which messages are retrieved from the blackboard-process message quene, the procedure call specified in the retrieved messages are processed, and the results are sent back to the original sender. The 
characteristics of message passing in a UNIX environment, as well as the implementation of the blackboard process as a loop, ensure that concurrent requests to the blackboard are sequentialised effectively.

As has been pointed out before, the $b b$-handler has to be able to preserve consistency of data on the blackboard. Inconsistencies may occur due to access of the blackboard by different, concurrently operating processes. Modifying blackboard access in order to be able to cope with concurrent access has been discussed elsewhere, e.g., in (Fennell \& Lesser, 1977; Ensor \& Gabbe, 1985). We summarise the various suggested adjustments for maintaining consistency of data on the blackboard:

1. A KS could ask the blackboard handler to forbid any other KS to read or write those units the KS intends to use. Such a lock could be restricted to only one unit, but could be extended also over regions of the blackboard, encompassing, e.g., part of a blackboard level, entire levels, and even complete blackboard panels.

2. A KS could add a tag to units it intends to use. As long as the tag is maintained, whenever other KSs make changes to a tagged unit, a message, specifying that the tagged unit has been changed, is sent automatically to the KS which placed the tag.

3. When a KS wants to make changes to the blackboard, the KS conld check whether the information on the blackboard coincides with its assumptions about the contents of the blackboard. If the assumptions are still valid, a request for a write lock is made and the blackboard can be changed. Of course this presupposes, among other things, the facilities as mentioned under 1.

'The first solution has one main disadvantage. It is often not possible to predict exactly which blackboard units are going to be accessed. This means that in some cases the regions requiring locks could become cxtensive, thus blocking blackboard interaction and consequently the execution of other KSs. A rlisadvantage of the second option is the high cost in overhead and the need for KS processes to monitor their message quenes for messages indicating modification of tagged blackboard units. The third option has also a disadvantage. Violation of assumptions is detected only after a KS has been executed. This disadvantage can be 
alleviated by checking the blackboard during execution to ensure that the assumptions still hold.

We have implemented the mechanism of the first option, which is necessary for using the third option. We distinguish between read locks and write locks. Read locks are requested by a KS when this KS is interested only in reading information from the blackboard or needs to search for information on a restricted portion of the blackboard. When a read lock is issued for a specified region, other processes may still read the contents of that region, but no process is allowed to modify that region. Write locks are requested, when a process needs to make a modification on the blackboard. A write lock for a region prohibits reading information in that region by any other process and modification of data in that region by any other process than the one holding the write lock. In order to avoid deadlock, it suffices to demand that a process releases all its previous locks before other locks (possibly including terminated locks) can be requested. It is possible to implement more sophisticated, but also more complicated, deadlock prevention or recovery mechanisms, but we did not expect to need them. Deadlock and ways to handle deadlock are explained in most textbooks on operating systems, such as (Lister, $1975)$.

It should be mentioned that in many applications, as in ours, the interference of interactions with the blackboard by different KSs can be kept low by only selecting KSs to operate concurrently which operate on disjoint sections of the blackboard. Thus, keeping the disadvantages of backboard access by concurrently operating KSs to a minimmm becomes a control problem. For this purpose, the KS descriptors in Blondic-[] contain references to the locks needed during their execution (sec ligwe 4.8$)$.

\subsection{Related work}

In this section we discuss other work in the area of parallel blackboard architectures. An early example of a parallel blackboard arclitecture is described in (Fennell \& Lesser, 1977). That contribution reports on an effort to take (a subset of) Hearsay-II and study ways to lum it into a parallel blackboard system. Several important issues were addressed, including a discussion of methods for ensuring data integrity, 
speed and efficiency measurements, and mutual influences of these two aspects. Unlike Blondie-II, however, the resulting system was designed for a specific application and for a homogeneous platform of processors.

Although Blondie-II was designed with a specific application area in mind, it was built as a general purpose, parallel blackboard framework. Another such framework is CAGE (Aiello, 1986; Appendix A). CAGE is more flexible with respect to the extent of concurrency allowed in an application. CAGE provides not only for parallel execution of $\mathrm{KSs}$, but also for concurrency within KSs by allowing rules to be evaluated and executed concurrently (see Appendix A for more details). Also, CAGE allows a user to specify the required amount of synchronisation during execution. Thus, for instance, the concurrent execution of KSs may start at the same time for all processors, or for each processor separately whenever a previously executed $\mathrm{KS}$ is ready and a next activity has been assigned. Blondie-JI does not provide facilities for synchronisation. Rather surprisingly, CAGE does not provide facilities for ensuring data integrity. This could be explained by a general notion among blackboard researchers that the blackboard architecture is inherently suited for dealing with incomplete and unreliable data. (Erman et al., 1980). We have argued, however, that additional facilities for ensuring data integrity are necessary in those applications where the existence of incomplete or unreliable data during the process can be damaging as is the case in robot control. Unlike CAGE Blondie-II is aimed at integrating differing components and supports applications, where not all KS's can be executed by all processors within the system.

A system which does am to integrate different linds of processors, in this case numerical and symbolic processors, is the KIM system (Knowledgebased Integration Manager, Winkelbauer, 1988).

CAGE and Blondie- II both differ from a score of parallel blackboard architectures. Both frameworks allow one processor to execute activities related to different KSs. Other blackboard architectures, in fact, stay closer to the blacliboard metaphor. Those architectures contain a number of concurrently operating systems which use a blackboard for communication and for deciding when to perform a certain activity. The subsystems in these architectures each perform their own cycle of alternately polling the blackboard for interesting information and perforning appropriate activities. Examples of this approach can be found 
in the systems CODGER for robot navigation (Shafer \& Stentz, 1987), the Distributed Problem Solving Kernel for engineering tasks (DPSK, Talukdar \& Cardozo, 1987; Talukdar et al., 1988), the office automation environment Basar (BAsic Software ARchitecture, Märtin \& Waldhör, 1988), the HOPES architecture for real-time processing (Dai et al., 1990; Dai et al., 1991), and the System Status diagnosis subsystem of Pilot's Associate (Pomeroy \& Irving, 1990; Pomeroy \& Stein, 1990). One consequence of an approach as taken by the designers of these systems is that control is much less of an issue: the concurrently operating subsystems are mostly responsible themselves for deciding when to do what.

The architecture of HCVM (Heuristic Control Virtual Machine, Fehling et al., 1987; Raulefs, 1987; Fehling et al., 1989) combines a parallel blackboard architecture with an object-oriented approach. HCVM complements the usual indirect method of invocation of activities via blackboard interactions with a direct form of invocation by means of objects and the exchange of messages between objects. Direct invocation of activities is deemed necessary to meet real-time constraints.

GEST (Roth et al., 1987; Tynor et al., 1987) is a general framework, based on the blackboard, that can be used to specify parallel blackboard systems (GEST also provides several mechanisms for knowledge representation, uncertainty reasoning, consistency checking, etc.). Here, KSs are continually executing programs, each specifying whether they are currently able to contribute and what the priority is for this contribution. A control component decides which KSs actually run at any given moment. However, in a way similar to Blondie-II, the total system runs on a single machine. Therefore, concurrency exists only at a conceptual level.

A similar approach to robot control, as presented in this chapter, can be found in (Pang, 1989; Pang \& Shen, 1990). These contributions describe a sequential blackboard architecture for intelligent robot control closely resembling Blondie.

\subsection{Evaluation}

Blondie-II is a tool developed for prototyping and simulating parallel blackboard applications. When Blondie-II was finished, work on using 
the sequentialblackboard shell Blondie for experimenting with control in a robot cell was already underway. The results of these experiments were such that Blondie-II was never actually applied to robot-cell control by the intended users of the system. This section reviews the reasons why the intended users did not apply Blondie-II and also indicates where the blackboard concept did prove useful. Finally, we address contributions made by the development of Blondie-II.

In several respects the blackboard-architecture approach to robot-cell control did not live up to the expectations of the intended. users. One of the basic requirements of robot-control architectures is a powerful mechanism for error recovery. Initially, we believed that the event-driven, opportunistic behaviour of blackboard systems makes them very much reactive in the event of exception detection. However, detection can still be a problem. For, in order to be able to detect an exception, one has to know what situation was to be expected. Especially in our projected robot-control application, where time plays an important role, predicting what situations should arise can involve sophisticated, temporal planning. Extra mechanisms would have to be supplied to address this issue.

Moreover, the intended users found that the idea of having a current world model represented on the blackboard did not work out in practice. In their opinion data on the blackboard can be the product only of preprocessing by other components in the architecture, typically by sensors and their interpretation systems. The resulting information on the blackboard is thus both compressed (due to preprocessing decisions by the interpretation systems) and delayed. The consequence of this is that the supervisor itself can never be as reactive and well-informed as is believed to be necessary. This observation has led the intended users of Blondie-II to another track of investigation (Penders, 1989), adopting a situated-action approach.

The observation that a situated-action approach (Agre, 1990) might solve problems arising in a blackboard approach is interesting for the following reason. There exists an obvious similarity between situated action and the blackboard model: the situated-action approach states that decisions with respect to the activity to perform next need to be made each time in a sea of data representing the complete situation at that time. Similarly, blackboard systems have a blackboard as represen- 
tation of the current situation which may be used extensively to make problem-solving decisions.

The strength of the current parallel architecture of Blondie-II can be summarised as follows:

- Blondie-II provides flexibility for:

- experimenting with different control strategies for concurrent blackboard systems,

- specifying different distributions of activities over various processors.

- Blondie-II is suited, at least in design, for integration of diverse components.

- The data-driven, event-driven nature of the blackboard architecture is better equipped for robot control than goal-directed, classical AI planning techniques.

The facilities for experimenting with different distributions is felt to be an especially useful contribution. It is believed, moreover, that the architecture could be transferred easily from a simulation environment to an environment in which actual execution of (sub)tasks is performed by other physical processors (the drivers and machines in Figure 4.2). However, one of the essential tasks of a robot-control cell, i.e., decision making, is not actually provided by the blackboard architecture. Although mechanisms are available for triggering activities at opportune moments, selecting the best activity or sequence of activity is still domain dependent and an open problem.

Although the main goal of creating a parallel blackboard architecture for robot-cell control would be the need for efficient use of several devices, and thus the need for speed up, Blondie-II in its present state cannot actually be used to achieve this speed up, as parallelism is simulated via time sharing. However, experiments with extending the communication facilities as provided in Blondie-II over a network of SUN workstations suggest that distributing virtual processors over such a network is straightforward and could lead eventually to actual speed up.

While the sequential blackboard shell Blondie was modelled closely after $\mathrm{BB1}$, which was designed for the application domain of planning, 
Blondie-II has been designed for a rather different application area, namely control. The main modifications of the original Blondie design necessary in this respect is regular removal of outdated information from the system, such as the history of events and executed KSARs, outdated units on the blackboard, etc. These were rather minor adaptations, illustrating the generality of the blackboard model. 


\section{Chapter 5}

\section{The Distributed Blackboard Framework Blondie-III}

Distributed Artificial Intelligence (DAI), or, more specific, distributed problem-solving; can be defined as the study of 'intelligent', semiautonomous systems cooperating to solve a single problem (Decker, 1987; Davis, 1980; Davis, 1982; Fehling \& Erman, 1983; Gasser, 1987; Smith, 1985; Sridharan, 1987; Huhns, 1987; Gasser \& Huhns, 1989; Bond \& Gasser, 1988). Thus, DAI is a combination of Artificial Intelligence and distributed processing. It adds to distributed processing in the sense that the 'intelligent' components in a distributed AI system make informed, 'intelligent' decisions about when to exchange which information or tasks with other components within the complete system. DAI is distinguished from the general field of Artificial Intelligence in the sense that distribution is used to obtain any number of advantages, such as speed up, integration of distinct components, or exploitation of a natural distribution in the problem domain, such as a logical or geographical distribution.

DAI systems are often presented as networks, where the nodes are formed by the semiautonomous subsystems and the connections exist as communication channels between the nodes. Hence the term distributed problem-solving network (or DPSN) to denote a DAI system. 
Literature in the field of DAI describes a number of examples of a blackboard architecture used in distributed problem-solving systems (e.g., Lesser \& Corkill, 1983; Craig, 1987b; Leao \& Talukdar, 1988; Jagannathan et al., 1987; Weihmayer \& Brandau, 1990). The blackboard architecture is suited for use in DPSNs for a number of reasons.

Consider a distributed blackboard architecture consisting of a network of blackboard systems. An example of such an architecture, consisting of four completely interconnected blackboard systems, is shown in Figure 5.1. Cooperation between the nodes (blackboard systems) in such a network can be achieved by the exchange of information and tasks. Information exchange between different nodes is achieved when a knowledge source (KS) present at one node may inspect the contents of the blackboard of another node and/or write data there. Then, KSs of different blackboard systems can interact in a way similar to the way KSs within a single blackboard system do. The blackboards in a network can be used not only to communicate information, but also to exchange tasks between the nodes: the appearance of new or modified data from one node on the blackboard of another node signifies a blackboard change in that other node and consequently may lead to the firing and execution of KSs in that other node.

The uniform method of communication between KSs via a blackboard allows blackboard systems to be used as 'building blocks' for a DPSN. However, this uniform method may work only if cooperation between systems can be defined in terms of the underlying blackboard communication view: the organisational definition of the blackboards in the nodes must be able to allow representation of information exchange relevant for communication with other nodes.

This chapter describes the design and implementation of a DPSN consisting of cooperating blackboard systems. Section 5.1 describes the design of Blondie-III, which is a framework for building distributed blackboard systems. Section 5.2 focuses on facilities in Blondie-III for communication between the nodes in a DPSN. In Section 5.3 a short discussion of similar distributed blackboard architectures is given. Blondie-III was used to implement a distributed blackboard-application prototype. The domain of this application, telecommunication-network configuration, is described in Section 5.4. The application prototype itself is described in Section 5.5. The clapter concludes with an evaluation. 


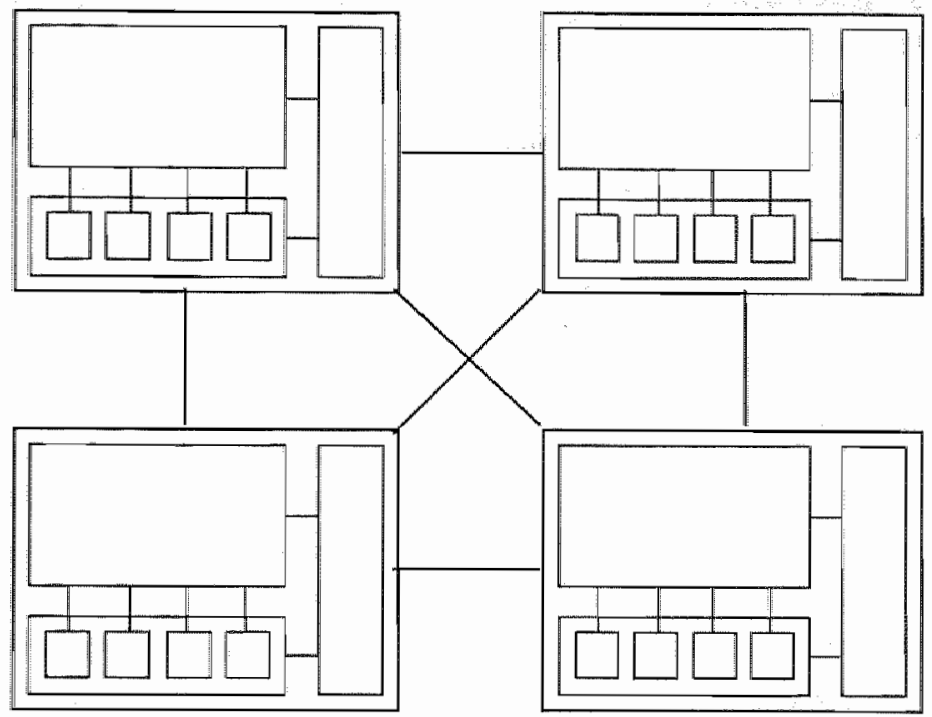

Figure 5.1: Example of a distributed blackboard architecture.

Material on the clesign of Blondie-III has been made available previously in (Florescu et al., 1988a). The telecommunication-network configuration application has been described in (Florescu et al., 1988b; Florescu et al., 1988c). Discussions on the network-configuration application have been published also in (Liempd et al., 1989; Liempd et al., 1990).

\subsection{Blondie-III}

The distributed blackboard framework Blondie-III is described in this section. Blondie-III does not support the development of all imaginable distributed blackboard architectures. Section 5.1.1 presents the restrictions which hold for distributed blackboard architectures based on Blondie-III. Systems developed in Blondie-III can be run in two distinct modes. In one of these modes only a single node is running, while communication with other imaginary nodes is simulated. In the other mode multiple nodes in a problem-solving process run concurrently and communication is achieved by message passing between the nodes. The reason for implementing these two different modes is given in Section 5.1.2, 
together with a more detailed description of how the two modes differ. Then, in Section 5.1.3, we describe which distributed blackboard architectures can be developed in Blondie-III and how architectures should be defined. Section 5.1.4 describes how the definition of an architecture is used by Blondie-III to run an application. Section 5.1.5 describes in what way the architecture of nodes in Blondie-III differ from the architecture of Blondie.

\subsubsection{Restrictions}

Several restrictions underly the design of Blondie-III. These restrictions influence the kind of distributed blackboard systems that can be built with Blondie-III. The restrictions are the following:

1. The hierarchy of the nodes in the DPSN is fixed during runtime. Nodes 'know' with which other nodes they want to communicate and cannot start a 'conversation' with new nodes.

2. The functionality of the different nodes is fixed as well. Nodes have an a priori set of KSS which cannot be expanded during a problem-solving session.

3. A node should have a priori knowledge of (relevant portions of) the blackboard organisation in nodes with which it communicates.

4. Communication functionality between nodes is limited. A node can obtain information from another node's blackboard, put data on it, and can request to be notified when specific events occur on the other node"s blackboard.

The first assumption is not a strict requirement in the current implementation: nodes can, in fact, dynamically change the lists of nodes to which their messages are sent, but the second and third assumption will prevent this usually from being necessary.

The second assumption precludes nodes 'learning' new IKSs or accepting KSs from other nodes. It need not, however, limit the dynamic adaptability of the system: if there is a set of nodes with the same KSs, the system may decide dynamically which node will perform some task (so, if there is a mechanical failure in one node, some other node may take 
over). Also, by giving a node a large set of generad and specific KSs, the actual functionality of a node may vary widely at runtime according to circumstances.

The third restriction determines the ease of communication between nodes in Blondie-III. The current implementation is general enough to permit nodes to communicate some information even without the prerequisite of a priori knowledge (see Section 5.2 .2 ), but usually at the expense of more overhead (extra KSs). When a node does not know the structure of levels in another node, the receiving node must interpret a message in order to determine its contents and to store its information at suitable locations on its blackboard. A node can use the message primitives in Blondie-III directly and more efficiently if it knows the structure of relevant levels in other nodes, or in fact has itself levels with an equal definition as corresponding levels in other nodes. In that case, the sending node can specify the correct location on the blackboard for the contents of the message.

The fourth restriction stating that our communication primitives are limited in functionality is based on two underlying assumptions regarding the interaction of nodes.

Firstly, we assume that communication channels are reliable in BlondieIII applications, so there is no need for specific primitives to ensure that messages arrive properly, such as acknowledgement of reception of messages.

Secondly, the absence of communication primitives for modifying existing data on blackboards of other nodes can be understood by examining our notion of a distributed environment composed of blackboard systems, which is analogous to a set of desks in an office. Consider each node as an employee who can communicate with other employees only by putting notes on their desks and by reading notes on his or her own desk and those of others. In an office, removing or changing notes on other people's desks may lead to anger and frustration, especially when done in inappropriate situations. Employees generally tend to leave other desks alone, as they know they do not have enough knowledge to judge the situation there. Human employees generally defend their desks against unwanted changes by others. By denying nodes the possibility of modifying the data on the blackboards of other nodes, we prevent unwanted interactions caused by the ignorance or 'bad' intentions of other nodes 
without the need for an active 'defence" system. Thus, we only need to allow nodes to be able to add data to and to read data from blackboards in other nodes.

\subsubsection{Different modes}

Due to the parallel and asynchronous character of the nodes in a distributed environment as implemented, developing an application in such a system poses special problems. Messages do not arrive always in the same order or at the same time, thus the behaviour of the complete system can vary even when the same input is given. This influences, e.g., the reproducibility of errors during application debugging. The following lists a number of considerations:

- The interactive facilities available to the user in Blondie, such as trace, inspect, and single-step mode (execution of one cycle at the time in the control loop) are useful tools during the development and debugging stages of an application. We wanted to keep these tools in Blondie-III.

- In Blondie-III a separate window is associated with each node. However, a user cannot follow easily traces in four or five simultaneously active windows. Interrupting a single node for inspection would mean disturbing the problem-solving process of the system as a whole, since the message traffic between the interrupted node and other nodes would be held up, whereas the messages between other nodes would continue normally. Interrupting all nodes simultaneously would require global synchronisation facilities, which we dicl not want to include.

- It should be possible to develop an application incrementally. For instance, we would like to be able to run and test the KSs of one node without all of the other nodes being fully present. However, if the behaviour of one node is clependent on messages received from others, how can such behaviour be tested adequately without the messages actually coming in?

We chose to provide facilities in Blondie-III for an application to be run in two different modes: single mode or multi mode. In single mode, only 
one node is actually running, i.e., executing its control loop. The interaction with other nodes is simulated: the runining node takes incoming messages from a file instead of actually receiving them in a message queue. Outgoing messages are recorded in files, one for each simulated node. These output files may be used later as input files for other nodes. In single mode the user has control over the full range of facilities for inspecting data within the blackboard system. That means, the user has the ability to set up and change the following options interactively:

- The user may specify how many cycles in the basic control loop must be carried out until the system can be inspected again by other means than by tracing.

- During each cycle of the control loop, the user can trace the following:

- Events created during the cycle.

- KSARs triggered during that cycle.

- The set of invocable KSARs at the end of the cycle.

- The chosen action, i.e., the KSAR which will be executed during the next cycle.

- Information on any blackboard panel or level at the end of the cycle.

- Messages received from other nodes processed during that cycle and messages sent during the cycle.

The tracing output can be sent to the screen or to different files.

- Additionally, a user can interactively inspect the state of the system whenever the control loop has been interrupted. The items which can be inspected are similar to the ones that can be traced.

In multi mode, all nodes are running and actual message passing takes place. The user is now unable to interrupt any node for inspection, but must wait for the problem-solving process to terminate. Before starting the system, the user can specify which items are to be traced during the entire session, and can have these traces sent to the window associated with that node, or to specified files. These files may be inspected. afterwards as a log of the problem-solving session. 


\subsubsection{Option files}

Option files in Blondie-III are files in which the configuration is specified for the organisation of the system in general and the nodes in particular. Blondie-III allows different configurations. The description of the options illustrates the possible flexibility.

We chose for file-driven instead of, e.g., menu-driven specification of these options because of convenience. It would be rather bothersome to enter the options for all the nodes by hand each time the system has to run, especially when those options are the same for several consecutive runs. Options are defined in files separate from files defining the blackboards, KSs, and user-definable procedures in order to increase surveyability. Blondie-III requires one system fle, containing the definition and initialisation of general system parameters, and one node file for each node. The system file defines the following:

- The names of the nodes in the DPSN.

- The mode for this run of the system.

- In multi mode, for each node, the name of the option file for that node. The contents of those option files are described below. In single mode, file names are given where messages to other nodes should be stored.

- Parameters specifying the information to be traced during the problem-solving process. This includes indicating where the results of traces are written (i.e., to the screen or to named files). The items which can be traced were listed in the previous section.

- Communication options for the entire system (see Section 5.2.2).

Each node must have a user-defined option file containing the definition and/or initialisation of parameters local to the node. Such parameters can override general system parameters defined in the system file and may include the following:

- Identifiers of the files in which the KSs, the blackboards, and control procedures for this particular node are defined. Once these file names are known, the corresponding definitions can be loaded into the nodes. 
- Sets of node names to which this particular node may send messages. The user may, e.g., define a class of nodes called neighbours, specifying node names of adjacent nodes in the DPSN. These classes define the topology and possible hierarchy of (communication) relations between nodes in the DPSN.

- Options for message handling in this node (see Section 5.2.2).

- File names where items traced for this node should be recorded.

\subsubsection{Start up and shut down}

Starting up a problem-solving system based on a set of semiautonomous nodes requires several steps. In Blondie-III, a dedicated procedure takes care of the following:

- General system parameters must be initialised. These parameters must be specified beforehand by the user in the system file, which will be read and processed by this procedure.

- Since all communication between nodes is performed by messages (see Section 5.2), message queues must be created for all nodes. In Blondie-III, the queue identifiers are made available globally. Thus, in principle, each node can communicate with all others.

- A process must be created for each node in order to run independently. Now the individual nodes can be started up.

Once the organisation of the complete system has been defined and initialised, each individual node has to be initialised. The definition of a node requires the following:

- Setting the parameters local to this node. Such parameters may override general system parameters defined before, and can be set Section 5.1.3).

- Starting the control cycle, which will select knowledge sources (KSs), execute KSs, and update the set of applicable KSs. 
Each node should contain a start-up $K S$ which is triggered by an empty trigger (the control unit will instantiate a $\mathrm{KS}$ with an empty trigger only during execution of the first cycle). This KS will be executed before all others, and may be used to initialise the problem to be solved. Execution of this KS can result in the appearance of new units on the blackboard which may trigger other KSs and so continue the problemsolving process. A node, designed to solve only specific subproblems in the overall task handed out by other nodes, can have a start-up $K S$ which actually does nothing apart from leaving that node waiting for messages from other nodes. Then, as soon as a message arrives, the message is processed, possibly creating events which may trigger KSs subsequently to start actual problem solving.

In Blondie and Blondie-II, the procedure Interpret-ksar, which is part of the control loop (see Chapter 3), executes a procedure to check whether a solution to the problem has been found. This procedure is called once every control cycle and determines whether the control loop should be terminated. In a distributed system, this simple arrangement is no longer sufficient. Only some nodes may have sufficient knowledge to decide that the problem as a whole has been solved. Also, in what way should a node behave which is designed to solve some subproblem in the total task? We may assume that such a node received a definition of this subproblem by a message from some other node. When it finds a solution for this problem, it should not leave the control loop completely, but stay available and react to questions from other nodes and resume the control loop when the definition of a new subproblem arrives. For each node a special KS must be defined which can perform appropriate activities when a solution to a (sub)problem is found:

- Other nodes can be informed that a solution has been found by sending messages to interested parties.

- The blackboard can be prepared for requests from other nodes, or for similar problems in the future. By using primitives provided for accessing the blackboard, data on the blackboard can be reduced to include only information useful to other nodes or for the solution of subsequent problems. This information must be such that it will not interfere with new problem-solving sessions. It is clear that in general a KS needs domain-dependent knowledge to be able to perform these activities. 
- The node may need to be prepared for new problems by removing any remaining triggered or invocable KSARs which otherwise might interfere with instantiations created during future problemsolving sessions. Removal of 'old' KSARs may be also necessary to prevent the node from executing low priority KSARs still present. Such KSARs could change the contents of its blackboard leading to the production of false information when other modes inspect its blackboard. The control loop is adapted in such a way that a node will be waiting for messages from other nodes when there are no triggered or invocable KSARs. In effect, the mode is left in a state in which the problem-solving process has been suspended because a solution to a (sub)problem has been found. Then, the problem-solving process can be resumed only by messages from other nodes.

\subsubsection{Modifications with respect to Blondie}

Blondie-III supports the development of DPSNs where the nodes are complete blackboard systems, each with an architecture derived from Blondie. The development of Blondie-III involved some modifications of Blondie and several additions to the original body of procedures. Modifícations are the following.

- As a result of changes in the module event-handler (see below), minor changes in the syntax of triggers and KS-descriptors were necessary.

- The basic control loop was extended with procedures for handling incoming messages, as is further described in Section 5.2 .

- The basic control loop was adapted further to deal with the new requirements for behaviour upon detecting that the current problem has been solved, as explained above.

- The top module in the Blondie system architecture, the command interpreter, had to be adapted to allow the system to be run in single mode or multi mode.

Apart from these modifications of already existing procedures in Blondie, Blondie-III also contains a number of additional procedures: 
- Procedures were added for sending and receiving messages among nodes in the system. These procedures are described in Section 5.2.

- A number of procedures were implemented for handling input and output in the windows associated with each node.

- A procedure for deleting data from a blackboard was added.

- Procedures were added for removal of triggered and invocable KSARs remaining after a (sub)problem has been solved and for preparation of the event-handling mechanism for receiving and working on new (sub)problems from another node.

\section{Changes in the module event-handler}

The basic control loop of the Blondie system executes three major procedures. One of these is Update-to-do-set (see Chapter 3). Part of its task is to take one by one the events which occurred during the last completed cycle of the control loop and to request from the $k s$-handler the KSs with matching triggers. The returned KSs are then given to the ksar-handler for creation of KSARs. These KSARs are added to the list of triggered KSARs. The implementation of this task has been modified for efficiency reasons. As a result, the notion of triggering event has been extended to be able to specify combinations of events that must occur before a KS is triggered. This way triggers are made more descriptive of the situation a $\mathbb{K S}$ is really interested in, alleviating the need for a sophisticated precondition verifying the triggering process. Since preconditions of triggered KSARs are evaluated during each cycle of the control loop, it is advantageous to minimize the time between the moment a KSAR is triggered and the moment the KSAR becomes invocable. Increasing the descriptive power of triggers contributes to this.

In earlier versions of Blondie, the above-mentioned task was implemented by comparing each event against the triggers of all KSs in order to find the appropriate KSs. For a single event, the time complexity of this algorithm is linear in the number of KSs. Since Update-to-do-set is executed for each cycle of the control loop, it is worthwhile increasing its efficiency. Using the fact that an event consists of a standard set of attribute-value pairs, it was not difficult to create a tree structure 
in which different events are represented by different paths and where KSs place markers in the tree to indicate types of events in which they are interested (see Figure 5.2). Markers close to the root denote general types of events, while those further away indicate more specific types. For example, the event

\section{$[[$ KIND new $]]$}

is less specific then the event

\section{[[KIND new][BLACKBOARD-LEVEL focus]] .}

When processing an event, a single path in the tree must be traversed, and all the markers along this path represent $\mathbb{K} S$ s triggered by this event. The time complexity of this algorithm is now independent of the number of $\mathrm{KSs}$, but linear in the allowed number of attribute-value pairs in an event, i.e., the depth of the tree.

By using communication primitives as mentioned in Section 5.2, other network nodes have the possibility of adding or removing message identifiers at appropriate points in this tree structure, effectively defining a. trigger to be matched with events in another node. Upon processing of events, these message identifiers are treated similar to KSs: when such an identifier is found along the path of an event, the corresponding message is retrieved to find the name of the interested network node and the triggering unit is sent to that node.

An example of such a tree structure used for processing events is shown in Figure 5.2. We note that in this example knowledge source $\mathrm{KS} 4$ will be triggered when a new unit appears on blackboard domain or on level focus on blackboard control. Knowledge sources KS1, KS2, and KS3 will be triggered by any new unit on blackboard control, while an event indicating that unit focus2 has been created will trigger the knowledge sources KS1, KS2, KS3, and KS4 and will send the contents of unit focus 2 to node $A$ in response to message 24 from that node.

As an extra result of this implementation, the user was given the possibility to explicitly use 'OR' connectives in the trigger specifications of KSs. An easily added extra was to allow the use of 'AND' and 'FOLLOWED$B Y^{\prime}$ connectives. For instance, a trigger such as 


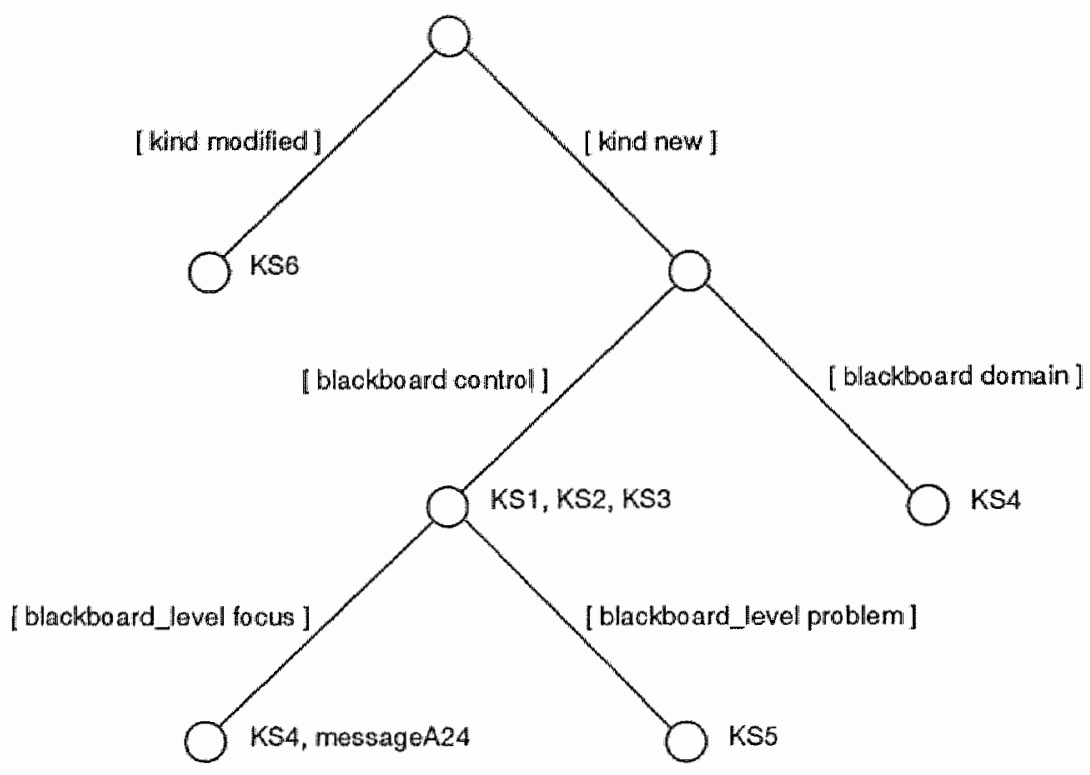

Figure 5.2: Example of the tree structure used for processing events.

[[kind new][blackboard-level problem]]

AND [[kind new][blackboard-level focus]]

means that the corresponding KS will not be triggered until both types of events have occurred in some order. Using 'FOLLOWED-BY' instead of 'AND' imposes restriction on the order of the events needed to trigger á KS.

\subsection{Communications}

In this section we give a description of the facilities available in BlondieIII for communication among nodes. Operating in a distributed environment necessitates a good exchange of information between the nodes contributing to the problem-solving process. The information must be sufficient in order to facillitate a meaningful interaction between the nodes. But, at the same time, the number of sent messages must be not 
too large, as each message potentially interrupts the problem-solving process within the receiving node.

The exchange of information in Blondie-III is, as in Blondie-II, implemented via UNIX System V operating system message passing facilities. Details of how these mechanisms are incorporated in the Poplog environment are described in (Velthuijsen, 1987b).

\subsubsection{Message handling}

The structure of messages was kept simple in order to reduce handling at the receiving end. Two kinds of messages are distinguished: nomal and special messages. The normal messages are used to exchange domain information, while the special message are reserved for exchanging control information, such as the announcement that a solution has been found to the overall problem. Special messages receive top priority.

Sending of information to other nodes results in the creation of units on the blackboards of these modes. Receiving information from other nodes is conceptually the same as reading information from the blackboard of another node. Thus, information exchange among different nodes is similar, conceptually, to local interactions with the blackboard.

\section{Communication primitives}

We defined a number of different communication primitives. These primitives can be used as procedure calls within the KS action parts. A starting point in designing these functions was the set of communication activities described in a report on the 1986 DAI workshop (Sridharan, 1987). During this workshop the issues of communication between nodes in DAI systems were discussed and a generic set of communication types was proposed. The following abstractions were listed: Inform, Request to do, Request to send, Command, Reply, Acknowledge, No-acknowledge, Offer, Agree, Refuse, Accept, Bid and Propose. We describe each of these abstractions and indicate whether we chose to implement a cer. tain abstraction in Blondie-III and why. The general restrictions as formulated in Section 5.1.1 influenced some of our choices. 
- Inform. This indicates sending of data. We implemented this via the procedure comm-inform by giving nodes the possibility of creating a unit on the blackboard of another node. The information is sent and the receiving node attempts to record it on its blackboard in a convenient place according to some specified options as will be explained in Section 5.2.2.

- Request to send. This is a request to another node to send back specific data. We identified three different situations in which to use 'Request to send' and provided for each situation with a different communication primitive:

comm-ask sends a message asking for all data matching a specified pattern. If there is no match, the receiving node is not required to return an answer. If there is a match, answers are returned by using the procedure comm-inform. Thus, no reference to the request is included in the answer.

comm-request sends a message asking for data matching a specified pattern. The difference between this and a comm-ask request is that, even when the pattern is not found, the requesting node receives a reply (when there is no matching data, the result is an empty list) and a reference to the original request is included.

comm-event sends a message asking to be notified when a specified event occurs during the problem-solving process of another node. When such an event occurs, the node sends back the units responsible for that event. This may occur as long as no comm-deny message has been sent. Sending a commdeny message means that the sender is no longer interested in events, specified by the comm-deny message. Thus, a commdeny message results in cancellation of an earlier request by comm-event.

- Request to do. This denotes a request to another node to undertake a specified activity. In a blackboard environment, activities are triggered by the appearance of new information on the blackboard. A blackboard system decides what to do next according to data present on the blackboard. Thus, sending data to a node can be used, indirectly, to request other nodes to undertake a certain 
activity. Therefore, a 'Request to do' can be implemented by using the primitive comm-inform and no dedicated primitive is provided in Blondie-III for 'Request to do'.

- Command. This is a command to another node to undertake a specific activity. A command is more imperative than a request to do, but otherwise similar. Thus, the same argument as for 'Request to do' applies here and no explicit primitive is implemented for 'Command' in Blondie-III.

- Reply. This abstraction facilitates a node to answer to a question posed by another node. A procedure for this should be implemented in order to be able to answer questions specifically needing a reply. We have implemented this abstraction as comm-reply and it is used to answer to messages sent by means of comm-request or comm-event.

- Acknowledge and No-acknowledge. These abstractions are intended for checking whether messages actually have been received at their destination. As we presuppose reliable communication channels between nodes in Blondie-III (see Section 5.1.1), we did not implement primitives for these abstractions.

- Offer, Agree, Refuse, Accept, Bid and Propose. These abstractions are used as part of the Contract Net Protocol (Smith, 1980). This protocol provides nodes with facilities to negotiate about tasks and resources. This is especially useful when nodes are not familiar with each other's capabilities or ongoing activities. However, we assumed that anyone who would develop a system with Blondie-III would have an overview of the resources and nodes within the system and how tasks should be divided over the system. This knowledge should be implemented in an application. Therefore, Blondie-III does not provide primitives for any of these abstractions.

We added the primitive comm-stop to the Blondie-III communication primitives mentioned in the above description. This primitive is used by a node to send a special message to the other nodes, indicating that these should terminate processing. The primitives that have been implemented in Blondie-III are shown in Figure 5.3. 


\begin{tabular}{|l|l|}
\hline primitive & description \\
\hline comm-rinform & $\begin{array}{l}\text { sends over data and no answer is needed } \\
\text { requests information from other nodes and } \\
\text { an answer is expected } \\
\text { asks for data but does not specifically expect } \\
\text { an answer }\end{array}$ \\
comm-event & $\begin{array}{l}\text { asks to be informed whether a specific event } \\
\text { will take place during the problem-solving } \\
\text { process of other nodes } \\
\text { sends information in answer to a received } \\
\text { message sent earlier by means of } \\
\text { comm-request or comm-event } \\
\text { discards a message sent earlier by } \\
\text { means of comm-event } \\
\text { sends a message to other nodes, saying } \\
\text { that they can shut down their system }\end{array}$ \\
\hline
\end{tabular}

Figure 5.3: Overview of communication primitives in Blondie-III.

\section{The blackboard-level communications}

The control unit is the only component in a node in Blondie-III often and regularly active. The blackboard itself, which is the actual subject of communication interactions, is never active. Interactions with the blackboard only occur as a result of KS and control unit activities. Messages are received in a node by the control unit, because of these observations.

It is important for a node is to be able to keep track of sent messages for which a reply is expected, because at the time a reply is received and handled by a node, the KS which posed the question originally will not be active anymore. For this purpose, we defined a dedicated blackboard level called communications. On this level we keep track of messages sent earlier by means of comm-request or comm-event, these being the only cases specifically needing a reply. The units on the blackboard-level communications contain information as indicated in Figure 5.4.

Whenever a message is sent by a $\mathrm{KS}$ using comm-request or comm-event, new units are created on level communications. When a message was sent by comm-request, the attribute TYPE has the value request until a 


\begin{tabular}{|l|l|}
\hline attribute & description \\
\hline NAME & unit name \\
IDENT & message identification \\
KS-NAME & name of the KS which sent the message \\
PATTERN & pattern which has to be matched \\
RESULT-UNIT & name of unit where the received reply is stored \\
RECETVE-LIST & list of nodes to which the message was sent \\
TYPE & equals request, completed, or event \\
\hline
\end{tabular}

Figure 5.4: Structure of unit on the blackboard-level communications.

reply is received. Then the attribute TYPE receives the value completed.

When a message is sent using comm-event, TYPE is given the value event. When the information requested via comm-event is not needed anymore, a comm-deny is sent. The unit corresponding to the related comm-event is changed: the original receivers of the comm-event messages to which a comm-deny message is sent are deleted from RECEIVELTST. When information is no longer expected from any of the receivers, the corresponding unit is deleted.

There are some aspects here needing more attention. Consider, for example, a situation where a node sends a comm-request message to more than one node, e.g., to node $B, C, D$ and $E$ and the sender is actually interested in information from $B$ or $C$ and from $D$ or $E$. In this case two units are created on level communications, having $B$ and $C$ in the list of receivers in the first unit and $D$ and $E$ in the list of receivers in the second unit. We now have an $O R$ relation between the receivers recorded in one unit and an AND relation between the receivers recorded in different units. Using this approach, RECEIVE-LIST will contain enough information about nodes which have received the message and will indicate also from which nodes a reply is necessary and from which nodes replies are sufficient.

An example illustrates this better. We imagine that $A$ has to solve a problem. $A$ has divided the problem into two subproblems. One subproblem is given to $B$ and $C$ to be solved by these nodes cooperatively, the other subproblem is given to the pair of nodes $D$ and $E$. Only when the two subproblems have been solved will $A$ be able to solve the entire 
problem. A will send a comm-request message to $B, C, D$, and $E$ asking for the solution of the subproblems. When an answer from $B$ or $C$ is received, the type of the corresponding unit will become completed. The same will happen in the second unit when an answer from $D$ or $E$ is received. When both units have an attribute type with value completed, $A$ will be able to solve the entire problem.

\section{Receiving messages}

As stated before, Blondie-III uses UNIX message passing facilities for the communication between nodes. Each node is given its own message queue. Messages to a certain node are sent to the corresponding message queue. At the beginning of each control cycle a node checks its message queue and interprets the messages in this queue, up to a certain limit. This limit can be defined by the user and is needed to prevent nodes from using too much time on message handling alone.

A node which has received a message (typically in the form of a blackboard unit) can put it directly on its blackboard and then use the information it contains analogously to when it had been derived within the node itself. A node receiving a unit has to know where on its blackboard this unit should be stored. In some cases, the node sending the message will try to specify the blackboard level on which the information must be recorded. This presumes, of course, that the nodes have some kind of knowledge about the structure of the blackboards of other nodes in the DPSN. In Blondie-III this is not really necessary. We made it possible to use a dedicated blackboard level to record incoming messages for which it is not exactly known where they belong. Also, the user has the option of specifying whether an attempt should be made to find corresponding levels for the messages or just record them directly on the dedicated level, or possibly even throw them away when no corresponding level can be found.

\subsubsection{Communication options}

As discussed in Section 5.1.3, Blondie-III uses a system file and node files for specifying different options for running an application. Some of 
these options define how communication between the nodes is organised. These options are the following:

- A choice must be made regarding the place where received information from another node will be stored on the blackboard of the receiving node. This is specified in the system file. The user may choose between the following three options:

1. Incoming messages are placed, if possible, on corresponding blackboard levels. If there is no such level, they are discarded.

2. Incoming messages are placed, if possible, on corresponding blackboard levels. If there is no such level, they are placed on a dedicated level, called comm-in.

3. All incoming messages are placed on level comm-in.

- The maximum number of messages read at the beginning of each cycle of the control loop is specified in the node files.

\subsection{Related work}

In this section some architectures similar to Blondie-III are discussed briefly. Other examples of distributed blackboard architectures were mentioned also in Section 2.4.

The basic architecture of Blondie-III resembles the initial architecture of Distributed Vehicle Monitoring Testbed (DVMT, Lesser \& Corkill, 1983; Appendix A). Interestingly, control in DVMT is goal-directed (Corkill \& Lesser, 1983; see also Chapter 8). Since the original design and implementation DVMT has been extended as a result of research in cooperation in distributed problem-solvers (Durfee et al., 1985; Durfee \& Lesser, 1987; Durfee et al., 1987). Other studies involving DVMT investigated, among others, meta-level control (Corkill \& Lesser, 1983; Hudlicka \& Lesser, 1984; Hudlicka \& Lesser, 1987) and goal relationships (Lesser et al., 1989b; Lesser et al., 1989a).

Following a similar design, the COPS system (Leao \& Talukdar, 1988) is also an environment for creating a DPSN. COPS uses a mechanism, called ambassadors for exchange of information among the nodes. When a node needs to read information from the blackboard of another node, 
the first node constructs an ambassador (a set of rules) in the other node. An ambassador represents the requesting node and sends interesting information back to the node it represents.

The CASSANDRA architecture (Craig, 1989; see also Appendix A) is a distributed architecture similar to Blondie-III. The nodes in CASSANDRA are formed by Level Managers (LMs). Each LM is associated with a blackboard level. A LM controls the KSs associated with a level and handles communication with other nodes in the system. This is basically the same architecture as in Blondie-III, except that the blackboard in a node in Blondie-III can contain multiple blackboard levels and panels.

Erasmus (Baum et al., 1989; Dodhiawala et al., 1987; Blevins et al., 1989) is a blackboard-generator language allowing the definition and implementation of a distributed blackboard architecture. Another example of a blackboard specification language which can be used for specifying distributed blackboard systems is ADL (Brajnik et al., 1988).

\subsection{A configuration task for a telecommunica- tion network}

Blondie-III has been used to implement a distributed blackboard system for the configuration of a telecommunication network. This section describes the configuration task for this network.

In the network users (terminals) are connected to applications (computer systems) through port selectors, called PACXs. The task at hand is to handle requests for connecting new terminals with specified computer facilities. Figure 5.5 illustrates an example of this problem domain.

A connection or line between two points indicates a physical connection, consisting of a cable and other required equipment. Each line can be used to carry a number of channels. The number depends on the different types of cable and equipment used.

The actual configuration task emerges when a user requests a connection with a computer system. Creating a connection implies that new channels must be found or created to meet the demands of the user. There are two ways to do this. The first possibility is to use existing capacity, i.e., using channels still available on existing lines. Another method is to 


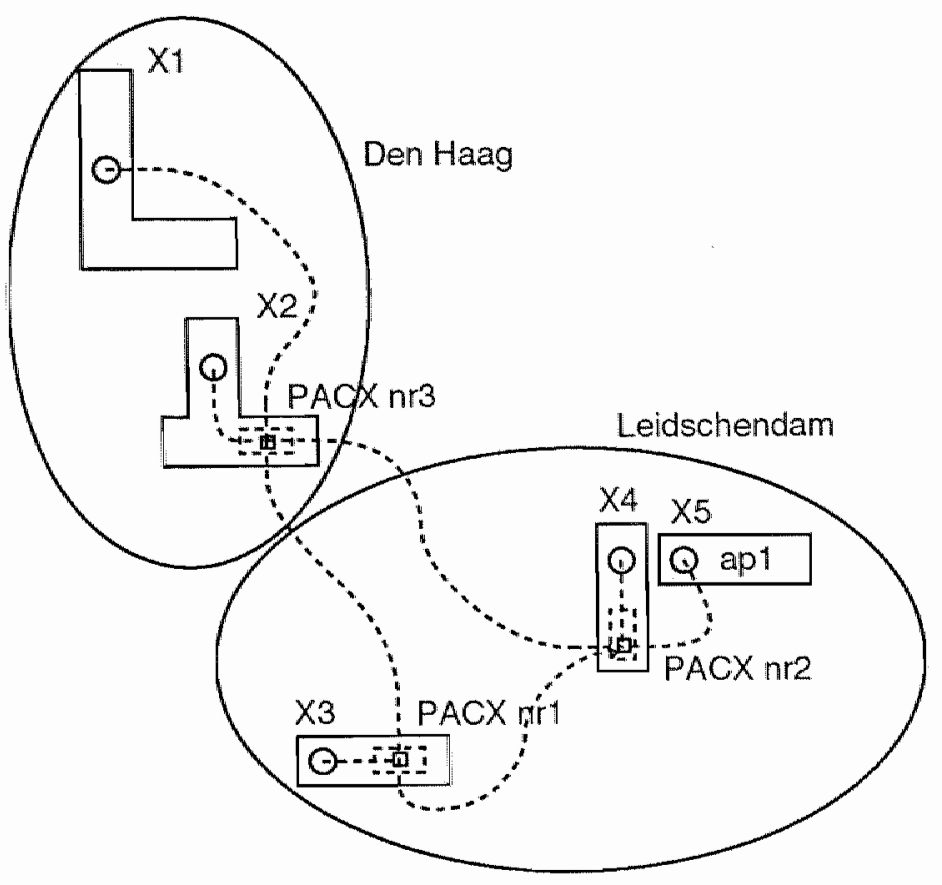

$x$.

O Buildings named "X." and computer systems

PACX ... PACXs named "..." ].

Possible connections (cables)

City limits of city named "..."

Figure 5.5: Topology of an imaginary district. 
construct, or plan, new lines. These two alternatives can be combined also, filling some of the existing available channels and planning the rest of the channels on new lines.

Planning a connection means choosing a type of cable, I/O card, modem, and multiplexer to obtain the desired functionality. There is a large variety of possibilities to do this. Many types of cables exist, and, for every type of cable, many different types of additional equipment exist that can be selected.

Not only are functional requirements important: constraints, such as budget, term of delivery, and reliability must be respected also. It is possible, e.g., that some types of cable, I/O cards, modems, or multiplexers are not available at the time the connection must be available.

There is also a problem with the availability of space when new equipment, such as $\mathrm{I} / \mathrm{O}$ cards, modems, and multiplexers, must be added to the PACXs in the network. A PACX is actually a rack with shelves on which the equipment must be placed. Each shelf has a limited size and contains a limited number of slots where equipment can be plugged in.

In short, several types of constraints, which may be contradictory, must be respected. Another fact making the task difficult is that the procedures used to perform such a task are not well known: at present, only human experts can handle the full extent of this task. All these considerations serve to illustrate the complexity of the network-configuration task. The purpose of the computer system described in the following section is interactive assistance of (human) network planners in selecting the right connections and equipment.

\subsection{Network-configuration implementation}

In this section, we describe the implementation in Blondie-III of a prototype system for the network-configuration task described in the previous section. Because of the complexity of the problem and the fact that the blackboard architecture supports incremental development, the problem was first reduced to a number of important parts. These parts (which were actually implemented) deal with filling existing capacity on lines and constructing new lines by selecting appropriate cables, multiplexers, and modems. What we left out was configuration within PACXs: 
selecting $\mathrm{I} / \mathrm{O}$ cards, deciding if there is any space for these on existing shelves, racks, and so on.

We describe how the problem-solving process was divided over several blackboard systems, discuss the tasks of the different systems, and briefly comment on how those tasks are implemented via KSs. An essential part of the implemented system is how it finds solutions satisfying the given constraints. In order to guide that process, rule-based expert knowledge was integrated into the KSs. This is described in more detail in Sections 5.5.2 and 5.5.3.

\subsubsection{Distribution of the system}

The problem-solving process has been distributed over five blackboard systems (nodes), coinciding with a logical distribution of the problem domain. The system contains a supervisor node which controls problem solving as a whole. The actual problem of creating a connection between a user and an application is divided into two or three similar subproblems corresponding with two or three sections of the total route: there is one section from the user location to the nearest port selector (PACX), one section from the application to the port selector nearest to that, and, if those port selectors are not the same, one section between the two port selectors.

Each of these sections has slightly different specifications and requirements. For instance, the standard specification for the number of channels needed for the section between two PACXs is only half of the total number of terminals, while there needs to be one channel for each terminal to the nearest PACX (this heuristic is employed by the human experts). Therefore, in the current implementation each section is handled by a separate node called a section node.

The fifth blackboard system is the network-database node. It contains information on the existing network and handles requests for information from the other nodes. Figure 5.6 illustrates the communications between these blackboard systems and between the blackboard systems and the user. 


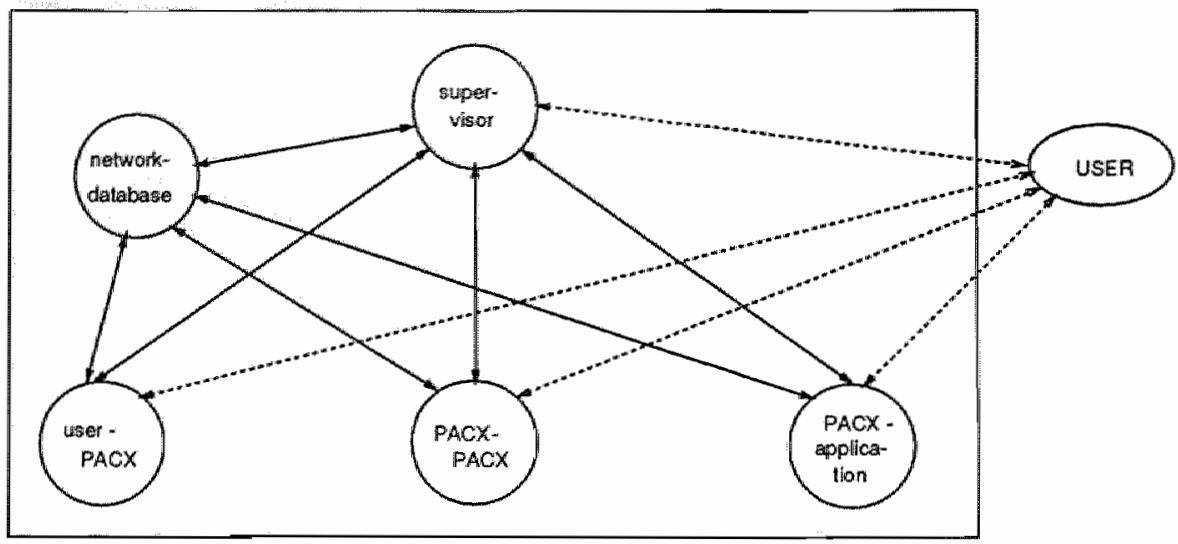

Figure 5.6: Communications in the distributed system. Solid lines indicate communication between the otherwise independent blackboard systems, while dashed lines indicate communication between blackboard systems and the user.

\section{The supervisor node}

The supervisor node has the following tasks:

- Initially, enough information must be extracted from the user and the network database to define a specific configuration task.

- Then, the problem must be distributed over the section nodes. These will take care of actual problem solving. During problem solving interaction between the supervisor and the other nodes may occur, but in the present system this is kept at a minimum deliberately (it may be added in later versions).

- When the section nodes have finished, the supervisor receives partial solutions from the different nodes. The total solution may be presented to the user for approval or integrated directly into the network database.

Each of these tasks is not very complex and can be handled by a small set of KSs triggered by easily identifiable events. 


\section{The section nodes}

Each of the section nodes progresses in two stages:

1. An attempt is made to find existing lines for the given section. If such lines exist with available capacity for additional channels, these are presented to the user. The user may choose to use channels on these lines. This choice is not done automatically. It is standard policy to reserve a number of channels for urgent requests that may be expected in the future. It is left to human operators to make assessments necessary in this respect.

Finding available channels on existing lines is called the stock problem.

2. If not all the required channels can be put on existing lines, new lines must be planned and constructed. After the user has made a selection of existing lines to use, the system plans a collection of lines which together satisfy the given constraints. When a candidate solution is found, it is presented to the user, who may choose to accept it or to have the system continue to construct another candidate solution. The system will be ready when the user is satisfied or the search for solutions is exhausted.

Planning new cables and equipment is called the planning problem.

The stock problem is straightforward and is handled by a number of strictly algorithmic KSs issuing requests to the network-database node, perform a number of computations, and present the results to the user. The planning problem, however, involves construction of solutions under possibly conflicting constraints (see below).

\section{The network-database node}

The network database is not a static repository of data. It contains KSs handling requests for information. For instance, sometimes the distance between two buildings is not stored explicitly on the blackboard of this node. A direct 'scan' of the blackboard by another blackboard system would not find the information needed. Therefore, a node which needs such information must put a unit on one of the blackboards of the 
network-database node specifying its request for data. A KS is triggered by the appearance of this unit and handles the request. This way, the blackboard architecture enables "procedure calls' from other nodes (as well as from other KSs within the node).

\subsubsection{Finding a solution}

The process of determining possible solutions for the configuration problem is a search process. Based on the requirements of the problem (the number of channels to plan, the speed required for each channel, costs etc.) a set of connections must be selected. A connection consists of a line with modems at both ends, and a set of multiplexers to be able to direct more than one channel over a single line.

Given the requirements of the problem, several line types are possible. Each line type can use a variety of modems from several brands with different specifications. Also, various brands and types of multiplexers are available. Selecting a multiplexer cannot be done independently from the choices for a modem and line, because, e.g., the synchronous output speed from a multiplexer must be within the range of speeds the modem can handle.

In short, each alternative connection consists of a set of dependent choices. The present problem-solving behaviour of experts in the domain of our application is as follows. They start by selecting a line type, then determine an appropriate modem and select a multiplexer for the configuration based on how many channels they want on that line. The specific order for making choices minimizes the size of the search tree. This ordering has the advantage that dependencies between choices are localised and that the most discriminatory choices are made first.

We briefly considered an implementation of this search process in a blackboard architecture as follows. A KS is triggered at each choice point. When executed, these KSs generate all possible selections at that point. For instance, every appearance of a line-type unit on the blackboard would trigger a KS named choose-modem which generates all possible modems suitable for that line type and the rest of the problem requirements. Each of the modem units could then trigger an appropriate KS which chooses suitable multiplexers for the configuration of the 
given line type and modem. The procedure determine-priority which determines the priority of invocable KSARs (KSs to be executed) should then see to it that the most promising $\mathrm{KS}$ is selected for execution.

This approach is not useful when the number of possibilities at a choice point is effectively infinite. Also, when we are interested only in finding a 'good' solution (as opposed to an 'optimal' one), an enormous amount of useless work may be done at each choice point by generating alternatives which will never be investigated. Therefore, we used another approach. In this approach at each choice point a KS is triggered generating only one alternative at a time. KSs generating such alternatives are called select-line, select-modem, etc.. We refer to these KSs as being of type select-.

The blackboard in our implementation contains levels for each of the components making up a solution. These levels are illustrated in Figure 5.7. The requirements and constraints for the connection to be found is represented on blackboard level problem. Connections are stored on level connection. A connection is determined by a structure (stored on level structure), indicating how many channels are routed over each of the lines in a proposed solution. Units on level structure also specify the proposed type of line. Selected modems and multiplexers are represented on levels modem and multiplexer, respectively. Level solution contains a full description of a proposed solution, including total costs, term of delivery, etc.

Figure 5.7 also illustrates how a solution is incrementally constructed on the blackboard. The nodes in the tree on the blackboard correspond to blackboard units. The arrows in the tree correspond with executed KSs. Additionally, these arrows represent links between the units on the blackboard. These links are used to indicate which nodes are included in the current (partial) solution; bidirectional links indicate inclusion, while unidirectional links indicate that a node has been considered and then discarded. The units contain not only links and information about the specific components they represent, but also additional information used to direct the problem-solving process.

Choice points in our implementation are marked by the change of attribute STATUS of units on the blackboard. A KS of type select- becomes invocable when the attribute STATUS of the triggering unit has value searching (i.e., a new alternative is needed). The general course of 


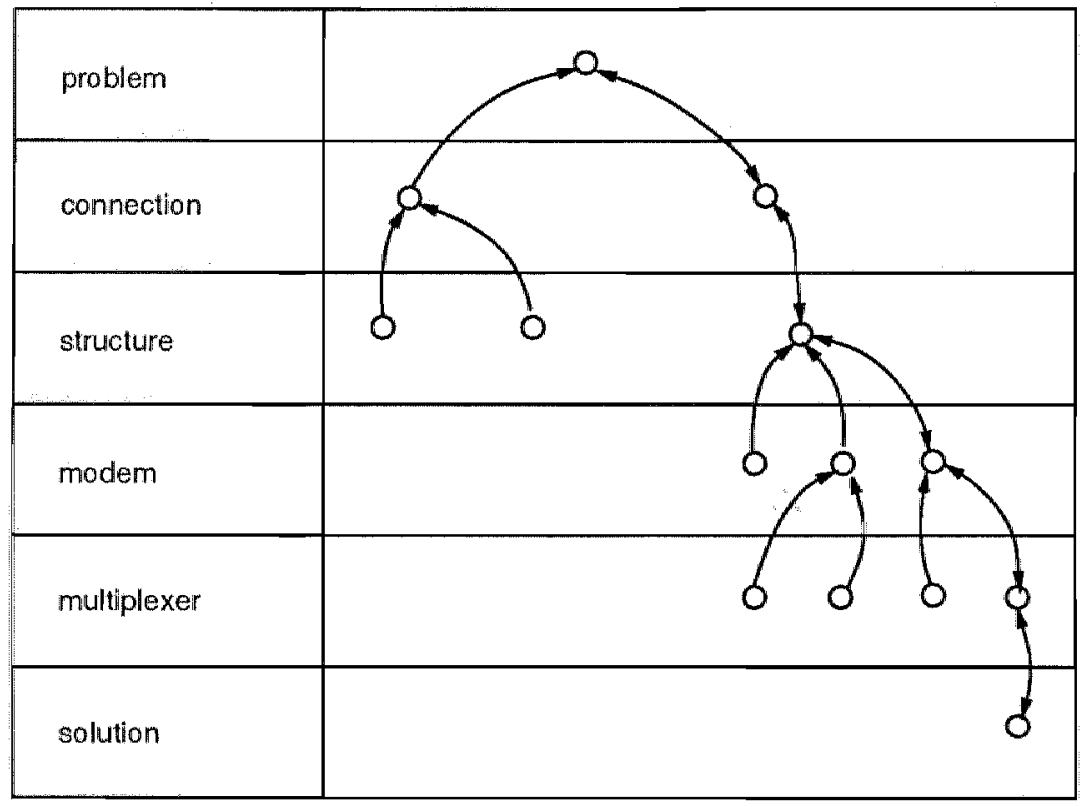

Figure 5.7: Finding a solution on the blackboard. 


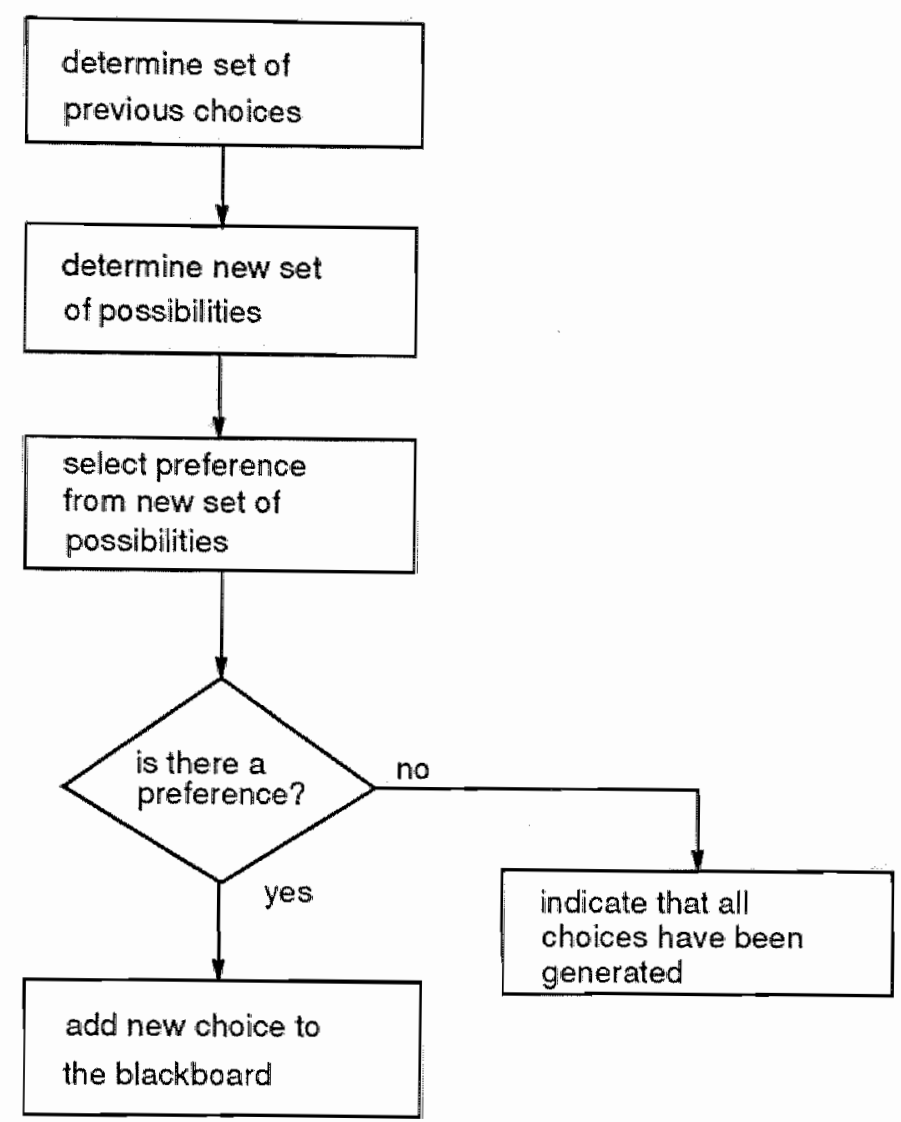

Figure 5.8: The flow diagram of select- KSs.

action for each select- KS is as follows (also illustrated in Figure 5.8).

- Determining the set of previous choices (which should now be avoided). This is done by simply checking the blackboard at the appropriate level. All alternatives created by a select- KS have a. link to the unit that triggered it.

For instance, select-modem searches level modem for all modem units which are part of the same partial solution triggering selectmodem.

- Determining a new set of possibilities takes into account previously 
made selections. Currently, the select-KSs use expert knowledge formatted as 'IF-THEN' rules to establish a new set of possible choices (see below).

KS select-modem, for instance, determines as the new set of possibilities all known modem types which fit the problem requirements (and which have not been examined before for this line type).

- From the set of possibilities, a single candidate must be selected. The KSs in our application again use expert knowledge in 'IFTHEN' format to do this.

- If such a selection can be made, a representation of the selected item is added to the blackboard. This automatically triggers KSs which may extend the partial solution once appropriate information is present.

To illustrate this, a multiplexer cannot be selected until the line type and the speed at which the modem is set are known. Therefore, KS select-mux is triggered by the appearance of a modem unit on the blackboard with status searching and becomes invocable only when an existing modem unit gets status searching.

- If no choice can be made, this path in the problem-solving process cannot be continued. The select- KS then sets the attribute STATUS of the triggering unit to unsolvable. The failing KS modifies the unit at the root of the solution tree at level problem, so that backtracking can be performed.

For instance, if all possible multiplexers for a given line type and modem type have been generated, either a new modem type or a new line type should be considered.

In the present application, a separate KS called check handles the cases when a new complete solution is found or when backtracking has become necessary. It examines the solution, or the point where finding a solution failed, and, based on heuristic rules, makes a decision what to do next.

An illustration of the kind of considerations involved in deciding how to backtrack is provided by the following. When a solution is found which meets all criteria of the problem except for delivery time, check will not backtrack to the choice point for modems or multiplexers (i.e., select a 
new modem or multiplexer), but will decide immediately to try another line type. The reason for this decision is the 'expert knowledge' stating that, usually, modems and multiplexers are readily available, while only lines need time to be delivered.

We notice that an important aspect of the problem-solving process is the fact that a solution should meet many criteria. The question arises which criteria should be relaxed, when no solution can be found satisfying all criteria. We used heuristics to guide the problem-solving process in these decisions too and allowed the user to indicate (and change) interactively which constraint is most important.

\subsubsection{Incorporating rule-based knowledge}

As seen in the previous section, the actual task of determining the possible choices is based on 'expert domain knowledge'. This knowledge cannot be captured easily in a single algorithm, but depends on many details of the problem. For a given detailed situation, however, we are able to express how a certain selection should be made. This can be adequately represented by IF-THEN rules. We have, therefore, cleveloped and implemented a simple rule-based mechanism, to store and apply the expert knowledge.

One way to incorporate both procedural and rule-based programming in a blackboard system is by defining some KSs as procedures and other $\mathrm{KSs}$ as rule-based systems. In this application, however, another approach was taken. When a KS needs to apply expert knowledge to perform its task, e.g., for finding all appropriate multiplexers given a specific line, modems, and structure, a procedure named engine (for inference engine) is called with the corresponding context. A context is a named list of rules suitable for a given situation. The context findmux, for instance, contains all the rules to find suitable multiplexers. The rules in a context may change the value of variables declared in the $\mathrm{KS}$ that called the procedure engine. The procedure engine may be called by any KS. Thus, one KS may use procedural as well as rule-based programming alternately. In our application a KS using rule-based programming typically declared and initialised necessary variables, called the procedure engine with the appropriate context, and, upon termination of the execution of procedure engine, used the changed values of its 


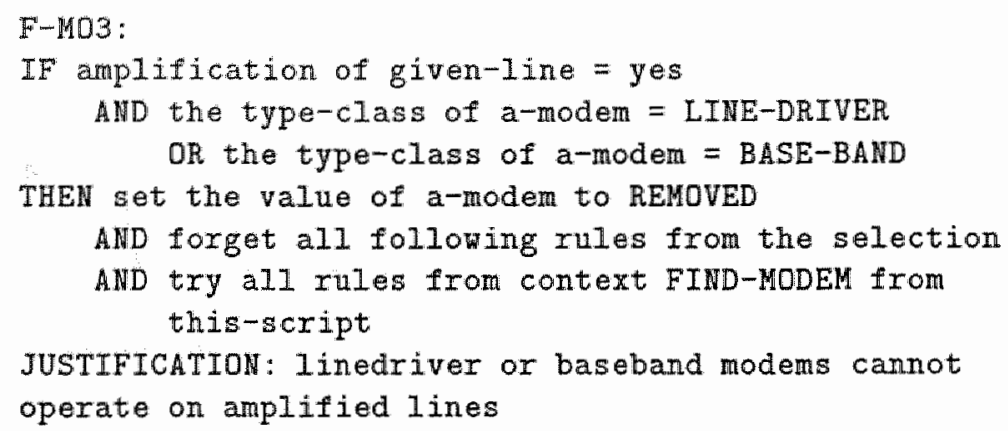

Figure 5.9: Example of a rule in the configuration application.

variables to record the contributions of this $\mathrm{KS}$ on the blackboard.

Each rule contains a condition part, an action part, and possibly a string clescribing the purpose of this rule. The inference engine tests all rules in a given context in order, and executes the activities collected in the action parts of the rules whose condition parts are satisfied. The condition part and action part of a rule use and act upon the variables of the procedure that called the inference engine. The action part of a rule may include a call to the inference engine to process rules in another context.

A procedure is provided to print the rules in a format close to 'normal English'. This procedure is not available while the application is running, since our concern was not explanation or justification to the user, but to provide support during development and maintenance of the application. A printed example of a rule is shown in Figure 5.9. This rule is contained in context find-modem which examines a list of modems to see whether they are appropriate in a given situation. The rule in Figure $5.9 \mathrm{can}$ be tested when the line type is already known. The forget activity in the action part makes sure that no more rules are tried for this particular modem. The try-all activity restarts the context on the remainder of the list of modems.

The rule-based inference engine is used by all select- KSs and the KSs make-structure and check. KS make-structure determines a suitable spreading of chamnels over lines. check uses rules to determine where to backtrack, when the problem-solving process halts in one direction, 
or to determine how good a solution really is.

The integration of procedural coding and rule-based programming has proved to be successful in our application. It enabled us to specify and implement different activities within the problem-solving task in a manner most natural for each activity. Yet, the resulting system performed sufficiently efficient and effective for our demonstration purposes.

\subsection{Evaluation}

We evaluate our experiences with Blondie-III and the development of a prototype for network configuration. This evaluation first discusses the network-configuration prototype. We conclude by indicating how Blondie-III supported the development of the prototype.

The network-configuration problem we have presented is complex. Selecting the right structure for a connection and then the right equipment to construct connections involves making many decisions. Construction is complicated by constraints that can be formulated by the user or posed by the availability of products.

By using the Blondie-III distributed blackboard-system framework, the solution to the network problem was implemented in a natural way. The existing spatial distribution of the network was mapped directly onto semiautonomous blackboard systems as nodes in a DPSN.

The resulting implementation performs well in the sense that the prototype finds solutions sufficiently efficiently and effectively for demonstration purposes. The current approach of incrementally generating alternatives guided by heuristics does not guarantee that an optimal solution will be found, but a good solution (if it exists) is found within an acceptable time. The current implementation handles also cases where no solution exists meeting all constraints by proposing in such cases solutions violating only 'weak' constraints.

Taking into consideration that the number of possible solutions can be very large, presenting all possible solutions for a given subproblem is not feasible. Generating and presenting the currently best solutions for different sections in parallel allow the user to postpone commitment to a solution for only one section until a view of a total solution has been 
obtained. We feel that handling the problem in a distributed way thus improved interaction with the user.

The current prototype system implements only part of the total problem domain. Enhancements are conceivable with respect to several aspects:

- Addition of constraints.

- Extending the configuration process to additional items, by considering necessary equipment within PACXs and buildings.

- Increasing interaction between the section nodes, for instance negotiation over budgets.

- Coupling of copies of this system for different districts.

We feel that this modular, distributed blackboard-architecture approach would prove helpful when interaction among nodes would be increased and the resulting system would be coupled with similar systems for different districts or with other systems. The modularity of the blackboard architecture is suited for adding more detail to the system or adding cooperation with other systems.

We now concentrate on how Blondie-III supported the development of the network-configuration prototype. We found that the communication primitives provided by Blondie-III were adequate in this case. However, we relied heavily on the assumption that the structural definition of the blackboards in the nodes is known during the design phase. This assumption enabled us to implement the exchange of information in a straightforward manner: information was sent from one node to another in a format which could be stored on the blackboard of the receiving node directly. It will be interesting to study how communication should be designed when the assumption does not hold.

We developed the nodes in our prototype separately, using the facilities provided by Blondie-III to operate an application in single-mode or in multi-mode. This enabled us to debug and tune each node without running into problems due to nondeterministically occurring interactions with concurrently executing nodes.

The use of blackboard systems as nodes in the network-configuration application proved beneficial in two ways. Firstly, the facilities for 
asynchronous communication between the nodes, provided by the blackboards, enabled us to incorporate in a simple way the exchange of information and tasks. Secondly, the use of a blackboard for storing partial solutions as well as the event-driven processing natural to a blackboard system provided good support for implementing the process of finding a solution. 


\section{Chapter 6}

\section{Formal Description of Blackboard Architectures}

In Chapters 3, 4, and 5, the blackboard architecture is mainly presented by example. The chapters each present one possible blackboard framework and illustrate its applicability by showing a specific application. In order to provide general answers to the central questions posed in Chapter 1 , we need to widen our perspective.

We have chosen to describe formally a number of blackboard architectures to help us find answers to these central questions. Formal description provides a means for carefully defining important dimensions in the design of a blackboard architecture and the variation along each dimension. Thus, formal description facilitates the effort of comparing diverse systems and architectures by providing a unified framework and terminology. The advantage of using formalisation is that it helps being complete and consistent.

This chapter provides definitions and notation for describing blackboard architectures formally. The resulting body of definitions forms a proposal for a formal model of the blackboard architecture. Descriptions of a number of existing blackboard architectures, using the definitions and notation presented here, can be found in Appendix A. Chapter 7 uses the wider perspective provided by the formal descriptions of several existing blackboard architectures as a basis for formulating answers to our questions. 
We stress that we are interested in describing blackboard architectures, not blackboard systems. Thus, we abstract from domain-specific issues, such as internal activities in KS action parts and specific control strategies.

This chapter is structured as follows. Section 6.1 presents the formalism used in our formal descriptions. Subsequent sections present formal definitions regarding the blackboard metaphor (Section 6.2), blackboard systems (6.3), control units(6.4), blackboards (6.5), and knowledge sources (6.6), respectively. Section 6.7 discusses some related work. The chapter concludes with an evaluation in Section 6.8. An initial version of this chapter has been published previously as (Velthuijsen \& Braspenning, 1990).

\subsection{Choice of formalism}

Two different approaches to developing a formal model for blackboard architectures seem viable. The first approach resembles the way architectures are specified within so called blackboard generators, such as Erasmus (Baum et al., 1989) and BSG (Silverman et al., 1989). These generators provide a language for specifying blackboard systems in predefined terms. A specification can then be compiled, together with the domain specific code (e.g., the code of KSs), into the desired system.

We observe that existing blackboard-system generators (regardless of their design generality) are not developed to enable the generation of the complete range of blackboard systems. There are always implicit and/or explicit design decisions implying restriction of one kind or another. A formal model of blackboard architectures should be more comprehensive than the specification languages of existing generator systems.

Moreover, such a specification language does not satisfy completely our purposes regarding a formal model of blackboard architectures. It does not allow to determine whether a certain architecture is blackboard-like or not. However, in order to be able to distinguish between blackboard architectures and others, we would need a model in which we can express also a set of 'non-blackboard' architectures. Since there exist parallel and distributed blackboard architectures, this inevitably leads to description mechanisms able to model concurrency. As to that, literature provides 
a multitude of different models and philosophies regarding the specification of distributed systems; in general, however, it is not yet clear which one is the best.

Several formalisms for specification of concurrent systems exist. We mention here algebraic formalisms, such as Milner's Calculus of Communicating Systems (CCS, Milner, 1989), Hoare's Communicating Sequential Processes (CSP, Hoare, 1985), and process algebras (ACP, Baeten \& Weijland, 1990), and model-oriented formalisms, such as Z (Spivey, 1988; Spivey, 1989), Petri-nets (Brauer et al., 1987), and event structures (Winskel, 1987). We preferred an algebraic formalism over model-oriented formalisms, since an algebraic approach is mathematically sound, while model-oriented formalisms are developed more intuitively. By choosing an algebraic formalism, we lost the possibilities afforded by Petri-nets and event structures to model causality, but gained the ability to compare systems more simply based on a sound notion of observation.

Of the algebraic formalisms, eventually we have chosen Milner's Calculus of Communicating Systems. Although CCS lacks some features of both CSP and ACP, we felt that these were not severe restrictions. An important aspect of CSP (not present in CCS) is the distinction made between internal and external choices. Although sometimes useful, this aspect makes the CSP constructor for parallelism much more complex. Moreover, ACP includes a constructor for process sequentialisation and has facilities for defining communication between more than two participants. Neither of these two facilities were needed in our efforts.

Our notation (and semantics) for dynamic aspects was derived from. CCS, although some simplifications and extensions were applied. Notably, we use the convention in Milner's book for sequential composition of processes, where control is passed by the special action done. However, the strength of CCS (such as proving equality or similarity of systems) still holds. Notation of dynamic aspects is presented in Table 6.1. The priority of the infixes (".", ";", "|", and " + ") is the same as the order in which these infixes are described: thus "I" has higher priority than "+".

The development of CCS was directed towards a formalism for describing abservable behaviour. As a consequence, it was not seen to be necessary to include in the CCS model constructions for specifying why a certain observation occurs and not another, thus CCS does not model causality. 
As we wish to be able to indicate in some cases how decisions depend on other aspects, we needed extra notational constructs for modelling such causality. Causality is specified separately by using notation for static aspects. Our notation for static aspects is based on a BNF-like notation (Backus-Naur Form). This notation is presented in Table 6.2.

\subsection{The blackboard metaphor}

Our construction of a formal model for blackboard architectures starts with a description of dynamic aspects of the blackboard metaphor. In subsequent sections we proceed with providing definitions of dynamic and static aspects of the components (blackboard, knowledge sources (KSs), and control unit), thereby accounting for causal relations. The terminology as given in the definitions in this and subsequent sections is reminiscent of that used in the literature. In some cases, however, our use might differ slightly from the one which is used regularly, if only because the use of terminology is not uniform for different blackboard architectures.

We start by considering the metaphor in its most basic form. We remind the reader that this metaphor describes experts (KSs) gathered around a blackboard. We introduce the following description of the blackboard metaphor $B B M$ :

$$
B B M=\left(B B\left|K S_{1}\right| \ldots \mid K S_{n}\right) \backslash L,
$$

where $L$ contains the labels signifying blackboard interaction:

$$
L=\{\text { read, write }\}
$$

The blackboard interacts with the KSs in read and write actions which may occur in any order. This may go on infinitely (practically, until no more KSs are interested in communicating with the blackboard):

$$
B B=\overline{\operatorname{read}} \cdot B B+\text { write } B B
$$

From the clefinition of the successor constructor ".", it follows that this equation allows for simultaneous blackboard interactions by different agents, provided no interactions start at exactly the same time. This is not a real restriction. 


\begin{abstract}
Name Names starting with a capital indicate an agent or subprocess.
name Names without a capital denote (atomic) actions. Atomic actions are observable, individual, recognizable 'events'. They refer to moments, so nothing is said about durations, beginnings, or endings of actions.
\end{abstract}

label Type-set names indicate communication opportunities (ports). Typically, label can only communicate with label, where a bar over the label indicates output and no bar indicates input. Input can be passed on to succeeding actions or agents via parameters (e.g., $\operatorname{read}(x))$. Variables are bound by its occurrence in an input label or at the left-hand side of a defining equation. In the first case, the scope is the agent description starting with that label; in the second case, the scope is the defining equation.

$\overline{\text { done }}$

The special communication $\overline{\text { done }}$ indicates that a process has terminated.

a.P The prefix constructor "." is used to indicate succession. $\alpha . P$ means: process $P$ follows action $\alpha$. The CCS formalism does not require $\alpha$ to have finished before $P$ starts; only that $\alpha$ has been observed before $P$ begins.

$P ; Q \quad$ The construction $P ; Q$ indicates that the process $Q$ will proceed after the process $P$ emits the special communication done.

$P \mid Q \quad$ The composition symbol "|" is used to indicate concurrency. $P \| Q$ means that processes $P$ and $Q$ occur concurrently. If $P$ and $Q$ are defined as a succession of (sub)actions, then the composition $P \mid Q$ stands for the summation of all possible orderings of these (sub)actions, where the original orderings in $P$ and $Q$ are preserved (e.g., if $P=a . b, Q=c . d$, then $P \mid Q=a . b . c . d+a . c . b . d+a . c . d . b+c . a . b . d+c . a . d . b+c . d . a . b)$. Thus $P$ and $Q$ are interleaved. We note that the composition $P \mid Q$ finishes only when both $P$ and $Q$ have finished.

$P+Q \quad$ Summation " + " indicates choice; $P+Q$ means: either $P$ or $Q$.

$P \backslash L \quad$ The symbol " $"$ is used to hide communication opportunities, i.e., (port.P) $\backslash\{$ port $\}$ can not communicate over labels port and port.

The agent 0 indicates an inactive agent.

Table 6.1: Notation for dynamic aspects. 


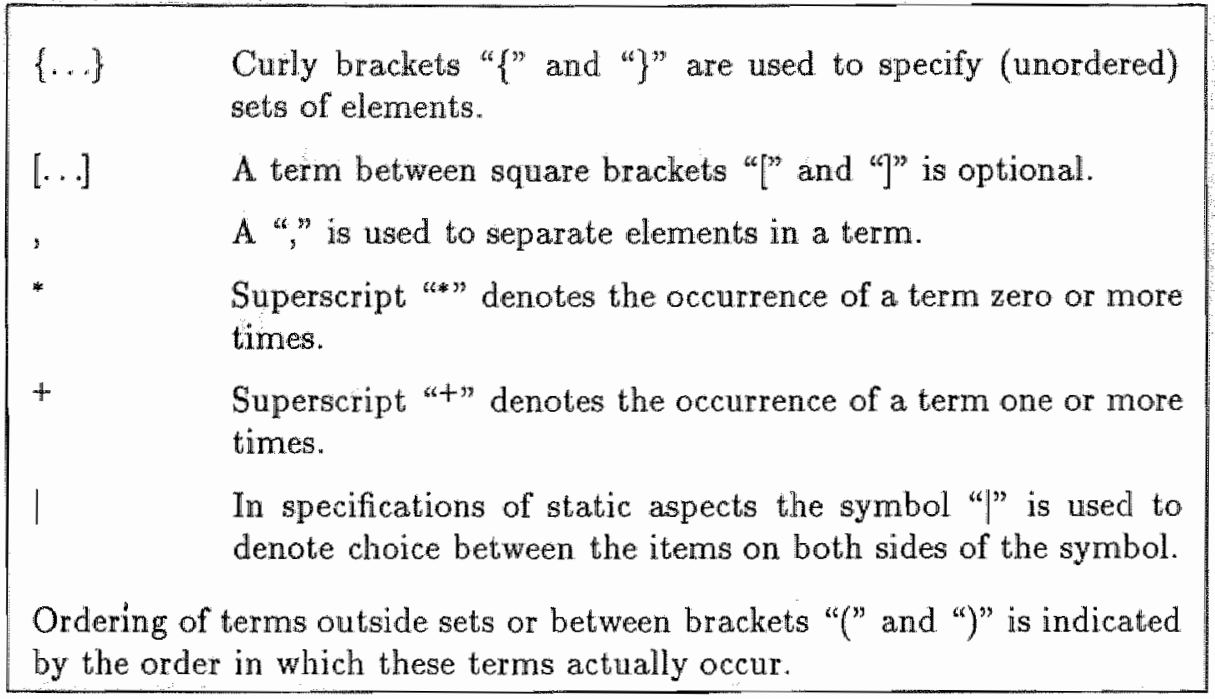

Table 6.2: Notation for static aspects.

KSs execute a cycle in which they alternately monitor the blackboard and perform - as soon as an interesting situation occurs on the blackboard - some activities ( $A c t_{i}$ ), which depend on the current blackboard state.

For a correct understanding of the equations in Definition 1 and further one should be aware of the following simple equational laws:

$$
\begin{aligned}
0+X & =X \\
0 ; X & =0 \\
0 ; X+Y & =Y
\end{aligned}
$$

Definition $1 \mathrm{~A} K \mathrm{KS}$ cycle is the cycle performed by a $K S\left(K S_{i}, i \in\right.$ $\{1, \ldots, n\})$ in the blackboard metaphor:

$$
\begin{aligned}
K S_{i} & =\operatorname{read}(x) \cdot\left(\operatorname{Match}_{i}(x) ; \operatorname{Act}_{i}(x) ; K S_{i}+K S_{i}\right) \\
\operatorname{Match}_{i}(x) & = \begin{cases}0 & \text { if there is no match } \\
\text { done } & \text { if there is a match }\end{cases} \\
\operatorname{Act}_{i}(x) & =\text { activity of } K S_{i}
\end{aligned}
$$

The variable $x$ indicates the current blackboard state and is used as a parameter for determining the applicability of the knowledge source $K S_{i}$ 
on this state $\left(\operatorname{Match}_{i}(x)\right)$ and possibly as a parameter for instantiating the KS. (We note that in this formulation a KS does not monitor the blackboard while performing a task. )

The actions taken care of by a KS may involve reading or writing the blackboard or other (internal) activities (int-act). Thus:

$$
\text { Act }_{i}=\text { int-act. } A c t_{i}+\mathrm{read} . A c t_{i}+\overline{\text { write }} \cdot A_{c t}+\overline{\text { done }}
$$

Here, we have not used the variable $x$ in this equation. We cannot model with our notation for dynamic aspects how the internal behaviour of a KS depends on its input (only that it depends). Moreover, we are not interested in internal behaviour of KSs from an architectural point of view.

The equations in this section can now be combined to give a definition. of the blackboard metaphor.

Definition 2 The blackboard metaphor describes an arganisation of subprocesses (KSs) cooperating by communicating via a global data structure (the blackboard). The dynamic interaction of these subprocesses and the blackboard is described by the following equations:

$$
\begin{aligned}
B B M & =\left(B B\left|K S_{1}\right| \ldots \mid K S_{n}\right) \backslash L \\
L & =\{\text { read, write }\} \\
B B & =\overline{\text { read. } B B+\text { write. } B B} \\
K S_{i} & =\operatorname{read}(x) \cdot\left(\text { Match }(x) ; A c t_{i}(x) ; K S_{i}+K S_{i}\right) \\
\text { Match }_{i}(x) & = \begin{cases}\frac{0}{\text { done }} & \text { if there is no match }\end{cases} \\
\text { Act }_{i} & =\text { int-act.Act } \text { int }_{i}+\text { read. Act }
\end{aligned}
$$

\subsection{Blackboard system}

In this section, we take the definition of the blackboard metaphor as a basis and derive a definition of a blackboard system.

We note that Definition 2 describes an organisation in which each KS monitors the blackboard and determines on its own whether it is applicable. There are as yet no constraints on available processors. When 
we assume that processing resources are limited and that $K S$ s have to be scheduled, we need to introduce a scheduler. The simplest form of scheduling involves no priorities (in most blackboard systems priorities are associated with KSs). The following represents this ( $m>0$ is the number of available processors; $m>n$ results in a system without scheduler):

$$
\begin{aligned}
& \text { Sched }^{(m)}=\underbrace{\text { Sched } \mid \ldots \| \text { Sched }}_{m \text { times }} \\
& \text { Sched }=\overline{\text { start }} . \overline{\text { end }} \text {.Sched } \\
& \text { Sched }{ }^{(0)}=0
\end{aligned}
$$

The labels $\overline{\text { start }}$ and $\overline{\text { end }}$ act as semaphores for the $m$ processors. The complementary ports are added to the definition of KS activity (6.2) in the following way:

$$
\begin{aligned}
\operatorname{Act}_{i}(x) & =\text { start. } A c t_{i}^{\prime} \\
A c t_{i}^{\prime} & =\text { int-act.Act } \\
t_{i}^{\prime} & \text { read. } A c t_{i}^{\prime}+\overline{\text { write. }} \cdot A c t_{i}^{\prime}+\text { end. } \overline{\text { done }}
\end{aligned}
$$

We remark that in Equations (6.3), no distinction is made between the processors. In some cases, however, not all processors can execute each and every KS. If so, one should discriminate between the labels start and end for different processors and create an appropriate summation for the corresponding discriminated labels start and end in the equations of (6.5).

Combining our original definition of the blackboard metaphor (2) with a scheduler leads to:

$$
\begin{aligned}
& B B S=\left(B B\left|K S_{1}\right| \ldots\left|K S_{n}\right| S \text { ched }^{(m)}\right) \backslash L \\
& L=\{\text { read, write, start, end }\} \\
& B B=\text { read. } B B+\text { write } B B \\
& K S_{i}=\operatorname{read}(x) \cdot\left(\operatorname{Match}_{i}(x) ; A c t_{i}(x) ; K S_{i}+K S_{i}\right), i=1, \ldots, n \\
& \operatorname{Match}_{i}(x)= \begin{cases}\frac{a}{\text { done }} & \text { if there is no match } \\
\text { if a match }\end{cases} \\
& \operatorname{Act}_{i}(x)=\operatorname{start} \cdot \text { Act }_{i}^{\prime}, i=1, \ldots, n \\
& A c t_{i}^{\prime}=\text { int-act.Act } t_{i}^{\prime}+\text { read. } A c t_{i}^{\prime}+\overline{\text { write }} \cdot A c t_{i}^{\prime}+\text { end. } \overline{\text { done }} \text {, } \\
& i=1, \ldots, n
\end{aligned}
$$




$$
\begin{aligned}
& \text { Sched }^{(m)}=\underbrace{\text { Sched }|\ldots| \text { Sched }}_{m \text { times }}, m>0 \\
& \text { Sched }=\overline{\text { start. }} \text { end.Sched } \\
& \text { Sched }{ }^{(0)}=\mathbf{0}
\end{aligned}
$$

In a single process implementation of a blackboard architecture, some applicable KSs may take a long time before they are executed. Meanwhile, other blackboard states may occur (as a result of other KS activities becoming executed) which enable execution of one or more of these KSs just as well. In many implementations this necessitates continuous monitoring of the blackboard for occurrences of matching blackboard states, even if a particular KS has already been annotated as applicable.

Definition 3 A knowledge source instantiation, or KSI, denotes a pair consisting of a KS and a context (bound variables, determined by the blackboard state in which this particular KS became applicable).

In this way, many different instantiations of a IKS may be applicable at the same time, each instantiation being determined by the blackboard state in which it arose. The pair of KS and context together form a (sub)task. However, not all blackboard architectures distinguish between different instantiations of the same KS. For instance, in cases where the action part of a $K S$ is not dependent on a context, but is a (sub)task in itself, a blackboard system may not need to distinguish. between different instantiations.

We can incorporate the notion of KSIs in Equations (6.6) and arrive at a definition of a blackboard system.

Definition 4 A blackboard system is a system which minimally consists of the subprocesses blachboard, knowledge sources, and scheduler with their usual meaning. Their dynamic behaviour is described by the following equations ( $n$ the number of KSs in the system; $m$ the number of processors available for execution of KSs):

$$
\begin{aligned}
B B S & =\left(B B\left|K S_{1}\right| \ldots\left|K S_{n}\right| \text { Sched }^{(n)}\right) \backslash L \\
L & =\{\text { read, write, start, end }\} \\
B B & =\text { read. } B B+\text { write. } B B
\end{aligned}
$$




$$
\begin{aligned}
& K S_{i}=K S_{i} \mid K S I_{i} \\
& i=1, \ldots, n \\
& K S I_{i}=\operatorname{read}(x) \cdot\left(\operatorname{Match}_{i}(x) ; \operatorname{Act}_{i}(x) ; \overline{\text { done }}+\overline{\text { done }}\right), \\
& i=1, \ldots, n \\
& \operatorname{Match}_{i}(x)= \begin{cases}\frac{0}{\text { done }} & \text { if there is no match } \\
\text { is a match }\end{cases} \\
& \operatorname{Act}_{i}(x)=\operatorname{start} . A c t_{i}^{\prime}, i=1, \ldots, n \\
& A c t_{i}^{\prime}=\text { int-act.Act } t_{i}^{\prime} \text { read. } A c t_{i}^{\prime}+\overline{\text { vrite }} \cdot A c t_{i}^{\prime}+\text { end. } \overline{\text { done }}, \\
& i=1, \ldots, n \\
& \text { Sched }{ }^{(m)}=\underbrace{\text { Siched }|\ldots| S c h e d}_{m \text { times }}, m>0 \\
& \text { Sched }=\text { start.end. Sched } \\
& \text { Sched }^{(0)}=0
\end{aligned}
$$

We note that Equations (6.7) can be transformed into Equations (6.6) by exchanging the term $K S_{i} \mid K S I_{i}$ with one of its constituents: $K S I_{i} ; K S_{i}$ (we note that $K S_{i} \mid K S I_{i}=K S I_{i} ; K S_{i}+K S_{i j} K S I_{i}$ ).

In these equations, $K S I_{i}$ is a pair in the sense that read $(x)$ defines the context and $\left(\operatorname{Match}_{i}(x) ; A_{c t}(x)\right.$; $\left.\overline{\text { done }}+\overline{\text { done }}\right)$ represents the functionality of the knowledge source.

All communication labels in Definition 4 are hidden from the environment; thus no external communication is allowed. Any effort to create an. implementation of this metaphor would have to include two important modifications of this model due to limited processing capacity.

The first modification stems from a limited number of available processors. Typically, there are not enough processors available to execute each applicable activity on a separate processor. Even worse: most implementations run on only one processor. This means not only that the processes of the blackboard and the scheduler have to be merged with each other and with the KSs, but also that mediating actions must be taken to handle KS activities which compete for the use of shared resources in a more sophisticated manner than by mere scheduling. This leads to a so-called control unit, defined as follows:

Definition 5 A control unit is the component within a blackboard sys- 
tem which mediates between $K S$ activities, competing for processing resources, and controls their execution.

A control unit limits the actions that can be observed for a blackboard system, compared with the generic description in Definition 4. A control unit can ensure, e.g., that KSs are executed only sequentially.

The blackboard architecture provides a data structure containing all relevant problem-solving information, namely the blackboard ${ }^{1}$. The information on the blackboard can be used together with information about the KSs to accomplish the aforementioned task (see Proposition 1 below).

A second modification is necessary usually due to the following. Computer programs are, unlike humans, not able to monitor a complete blackboard efficiently. This has led to an important adaptation in many blackboard systems. In those systems KSs do not monitor the complete blackboard, but rather changes of the blackboard.

Definition 6 The set of all data on the blackboard at any given moment is colled the blackboard state at that moment.

Definition 7 Let $s_{1}$ and $s_{2}$ denote two subsequent blackboard states. Then, a change of the blackboard state is called a state change and can be described as an ordered pair $\left(t_{1}, t_{2}\right)$, where $t_{1}=s_{1}-\left(s_{1} \cap s_{2}\right)$ and $t_{2}=s_{2}-\left(s_{1} \cap s_{2}\right)$ (i.e., $t_{1}$ represents the data in $s_{1}$ that did not persist, and $t_{2}$ represents the data that is new in $\left.s_{2}\right)$. We represent a state change by $\Delta\left(s_{1}, s_{2}\right)$.

Evidently, state changes are the result of communication between the blackboard and KS processes over port write. However, as we will see, monitoring only state changes is usually not sufficient for determining the applicability of a KS.

\footnotetext{
"We assume that all information relevant to the problem-solving process is in fact represented on the blackboard.
} 


\subsection{Control unit}

In this section we examine the activities of a control unit. At first, we concentrate on control units for sequential blackboard systems. Then, we proceed by defining possible control units in concurrent blackboard systems.

\subsubsection{Control unit in sequential blackboard systems}

Let us now examine what the definition of a blackboard system becomes if we assume that only one processor is available for all processes. Then the processes must be merged into one process to form a sequential system. One way of merging is via time sharing, but this is of no interest to us, as it cannot be controlled on an architectural level. The smallest items for merging are the communications and processes in the preceding equations, such as read, $M a t c h_{i}$, and $A c t_{i}$. The following simplifications of equations in Definition 4 are immediate. No extra processors for $\mathbb{K S}$ execution are available, so the scheduler becomes the null action. The blackboard is no longer treated as a separate process. In the following we exchange the communication labels $\overline{\text { read }}$ and write and their complements with actions read and write.

A first definition of the behaviour of a sequential blackboard system (without actually merging the processes) is given by the following equations:

$$
\begin{aligned}
S B B S= & K S_{1}|\ldots| K S_{n} \\
K S_{i}= & K S_{i} \mid K S I_{i} \\
& i=1, \ldots, n \\
K S I_{i}= & \operatorname{read}(x) \cdot\left(\text { Match }_{i}(x) ; \text { Act }_{i}(x) ; \overline{\text { done }}+\overline{\text { done }}\right), \\
& i=1, \ldots, n \\
\operatorname{Match}_{i}(x)= & \begin{cases}\frac{0}{\text { done }} & \text { if there is no match }\end{cases} \\
\text { Act }_{i}= & \text { int-act.Act } t_{i}+\text { read.Act } t_{i}+\text { write.Act }+\overline{\text { done }}, \\
& i=1, \ldots, n
\end{aligned}
$$

Abstracting from the activities inside a KS, we are now interested in finding all reasonable merges of processes $K S_{i}$ ( $K S$ cycles), i.e., sensible 
orderings of read $(x), \operatorname{Match}_{i}(x)$, and $A c t_{i}(x)$. (We remind the reader here that the combinator "|" indicates all possible orderings that preserve the orderings within the constituents.) However, some semantical restrictions apply to all possible merges. We mention three of these restrictions.

Firstly, the subprocess $M$ atch $(x)$ should follow a read action before a $\mathrm{KS}$ is executed in order to guarantee that the match indeed involves the current blackboard state. Secondly, the action read(x) - and thus $\operatorname{Match}_{i}(x)$ for a certain $i$ - should not be repeated until at least one KS has had the chance to be executed and possibly to change the blackboard. Finally, we note that the action read $(x)$ in the equation for $K S I_{i}$ does not depend on a certain KS (hence no subscript $\dot{z}$ was used). Since all $K S_{i}$ processes are merged into one process, a repetition of $\operatorname{read}(x)$ (for different $K S_{i}$ ) is useless until at least one $K S$ has been executed. These restrictions leave little room for variation. The only satisfying sequences are represented by repetitions of partial sequences of the form:

$$
\operatorname{read}(x) \cdot \text { Match }_{i_{1}} ; \ldots ; \text { Match }_{i_{k}} ; \text { Act }_{j_{1}} ; \ldots ; \text { Act }_{j_{1}},
$$

where $\left\{i_{1}, \ldots, i_{k}\right\} \subseteq\{1, \ldots, n\}$ and $\left\{j_{1}, \ldots, j_{l}\right\} \subseteq\{1, \ldots, n\}$, n being the number of KSs. Figure 6.1 illustrates the merging of KS cycles into this single cycle, called the control cycle. The introduction of 'selection' in the merged cycle in Figure 6.1 is explained below.

Since it is not meaningful to repeat a specific subprocess $M$ atch unless a KS has been executed, we cannot use the same definition for Match as before (implying abortion of execution of subsequent subprocesses in a loop when a match fails). From now on we use the activity match to indicate this distinction. Thus, Equation (6.9) becomes:

$$
\operatorname{read}(x) \cdot \text { match }_{i_{1}} \ldots \text { match }_{i_{k}} \cdot \text { Act }_{j_{1}} ; \ldots ; A_{\text {act }},
$$

Definition 8 The control cycle in a sequential blackboard system is formed by a cyclic recurrence of the following steps:

- Examination of the state of the blackboard.

- Determination of applicability of knowledge sources in view of the current blackboard state.

- Execution of (some) applicable knowledge sources. 

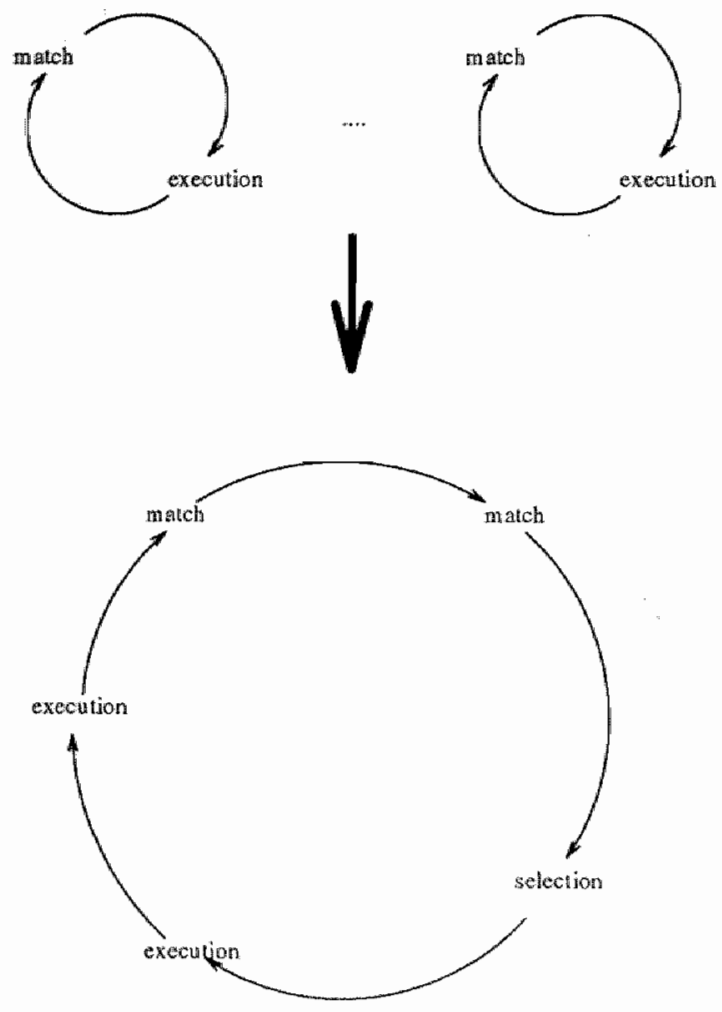

Figure 6.1: Merging the KS cycles into one control cycle. 
A special control cycle is defined by the cyclic expression:

$$
\begin{aligned}
S B B S^{\prime}= & \operatorname{read}(x) \cdot \operatorname{match}_{1}(x) \ldots \operatorname{match}_{32}(x) \cdot K S_{i(x)} ; S B B S^{\prime}, \\
& i(x) \in\{1, \ldots, n\},
\end{aligned}
$$

where only one $\mathrm{KS}$ is executed per cycle. This equation comes close to the basic control loop, as used in BB1 (Hayes-Roth, 1985), but an important issue has yet to be addressed, i.e., termination of the control cycle.

We have not yet examined in the description of the metaphor how a blackboard system terminates. From the equations in Definition 4, it follows that the blackboard system will stop when no more communication opportunities arise. It also stops when the KS processes eventually enter a non-terminating loop of alternately reading the blackboard and determining applicability of the KS based on the current blackboard state; if all KS processes are so trapped, no KSs will execute and thus no changes on the blackboard will occur, leading to livelock (a situation where subprocesses are busy without actually proceeding). In this situation, the scheduler will be waiting idly for a KS process to announce its interest in being executed.

Typically, there exists a number of ways for a blackboard system to terminate at a certain time. The first way occurs when there are no applicable KSs left. This way of termination is inherent in blackboard systems. Only for specific blackboard systems is it possible to guarantee that this does not occur.

The second way is to halt a system when a solution has been found. This defines the way we would really like a blackboard system to terminate, because it inplies that the problem-solving task is completed. In order to implement this, an action is needed to check for solutions and hence to decide eventually to stop the blackboard system.

A third way for a blackboard system to stop execution occurs when all running KSs have become unable to complete their tasks, e.g., when they are deadlocked. Implementations of concurrent blackboard systems easily rum into situations of deadlock when no precautions are taken.

We are now in a position to define the dynamics of a control unit more precisely. We adapt the control cycle presented in Biquation (6.10) to include checking whether a solution lias been found. Furthermore, the 
action $\operatorname{read}(x)$ is now included in the other actions (each action can read the blackboard to obtain information relevant to that action). We also introduce the separate action sel for selecting which activities to execute during each cycle. The following proposition states what actions constitute a control cycle.

Proposition $1 A$ control unit (CU) in a sequential blackboard system is defined completely by the following sequence of actions: determination of applicable KSs (matchi's), selection of KSIs (sel), execution of KSIs $\left(e x_{j}\right)$, and a stop criterion (stop):

$$
C U=\operatorname{match}_{i_{1}} \ldots \text { match }_{i_{k}} \cdot \text { sel }_{.} e x_{j_{1}} \ldots e x_{j_{l}} \text {.stop. }(\overline{\text { done }}+C U)
$$

In this equation, $i$ ranges over the KSs in the system ( $k \leq$ the total number of KSs in the system), while $j$ ranges over the currently generated and not yel executed KSIs ( $j \leq$ the total number of generated and not yet executed KSIs).

Here, the cyclic succession is represented by recursive definition of $C U$. The choice depends on the outcome of the action stop.

We now examine the actions $a p p$, sel, ex, and stop in more detail. We do this by viewing app, sel, ex, and stop as mappings. Hence, in the definitions describing these four actions, we abandon the CCS notation momentarily. The symbol BS denotes the set of all possible states of the blackboard, whilst the symbol $K S$ refers to the set of all knowledge sources used, and the symbol KSI represents the set of all possible knowledge-source instantiations.

Definition 9 Applicability of a $K S\left(K S S_{i}\right)$ is decided by the mapping match :

$$
\text { match }_{i}: B S \longrightarrow\{0,1\},
$$

such that matchi $(b s)=1 \Leftrightarrow b s \in$ condition $_{k s_{i}}(\subseteq B S)$,

(condition $k_{\text {s, }}$ being the set of blackboard states in which a KS becomes applicable, see also Definition 23).

Definition $10 \mathrm{KS}$ selection is a mapping (sel) determining a subset of an existing set of KSIs. This subset is selected for exccution. KS selection uses the current state bs of the blackboard:

$$
\text { sel: } \mathcal{P}(K S I) \times B S \longrightarrow \mathcal{P}(K S I), \text { where: }
$$


$\forall S \subseteq K S I, \forall b s \in B S, \operatorname{sel}(S, b s) \subseteq S^{2}$

Here, the domain of the mapping sel in Definition 10 can be restricted in most blackboard systems. Examples of selection mappings with restricted domains are:

- select that KSI whose corresponding KS has the highest (static) priority (a simple best-first strategy),

- select that KSI most recently generated (a depth-first strategy ${ }^{3}$ ).

- select that KSI generated earliest during the problem-solving process (a breadth-first strategy).

These examples use only information from the KSIs. However, we note that all information can be used that is present in the set $\mathcal{P}(K S I) \times B S$. This explains, partly, the power of the blackboard architecture.

Definition 11 The execution of a $K S\left(e x_{j}\right)$ coincides with the execution of the action part of the corresponding $K S_{j}$ (i.e., $K S_{\text {action }}$ ) and forms a mapping from one blackboard state onto a (next) blackboard state:

$$
e x_{j}: B S \longrightarrow B S,
$$

resuiting in one state change or several (more elementary) state changes.

Usually, both the domain and the image of this mapping can be restricted rather significantly, because a KS often uses only information from one blackboard level and writes intermediate results to one (another) blackboard level. Yet, a general restriction cannot be specified, at least not without first examining the semantics of the blackboard process.

Definition 12 The stop criterion (stop) is a mapping which assigns a Boolean value to each state of the blackboard (depending on whether a solution to the problem to be solved is present on the blackboard):

$$
\text { stop }: B S \longrightarrow\{0,1\}
$$

\footnotetext{
${ }^{2} P(K S I)$ denotes the powerset of $K S I$, i.e., the set of all subsets of $K S I$.

We note that in this depth-first strategy previously generated KSIs might become inapplicable during the execution of other KSIs, thus dynamically pruning the original tree of IKSIs.
} 
Usually, the domain of this mapping can be restricted to just one blackboard level or even to just one blackboard unit.

In existing blackboard shells and systems, the diversity in the allowed complexity of the four mappings described in Definitions 9-12 is rather broad. Further analysis should provide a better understanding of what is possible and what the semantic consequences are of any particular form.

\subsubsection{Control unit in concurrent blackboard systems}

The discussion of control units in the previous section was based on the assumption that only one processor is available for all activities. By relaxing this assumption, we obtain concurrent blackboard architectures. This does not necessarily also mean that matching and selection of tasks need to be distributed over separate processors (still assuming that not enough processors are available for all applicable KSs). In Definitions 13 and 14, we present two distinct approaches for defining a control unit in a concurrent blackboard system.

Definition 13 The control unit in a distributed-control blackboard system (indicated by $D C U$ ) is described as follows ( $m>0$ the number of available processors for execution of KSs):

$$
\begin{aligned}
D C U^{(m)}= & C U_{1}|\ldots| C U_{m} \\
C U_{r}= & \text { match }_{r i_{1}} \ldots \text { match }_{r i_{k_{r}}} \text {.sel. } \\
& e x_{r j_{1}} \ldots e x_{r j_{r}} . \text { stop. }\left(\text { done }+C U_{r}\right), \\
& r=1, \ldots, m
\end{aligned}
$$

Definition 13 describes a system of several concurrently executing control cycles, each activating and selecting its own set of KSs. This is illustrated by Figure 6.2.

Definition 14 The control unit in a parallel blackboard system (indicated by $P C U$ ) is described as follows ( $m>0$ the number of available processors for execution of $K S$ s):

$$
P C U^{(m)}=\left(C \mid \text { Proc }_{1}|\ldots| \text { Proc }_{m}\right) \backslash P L
$$



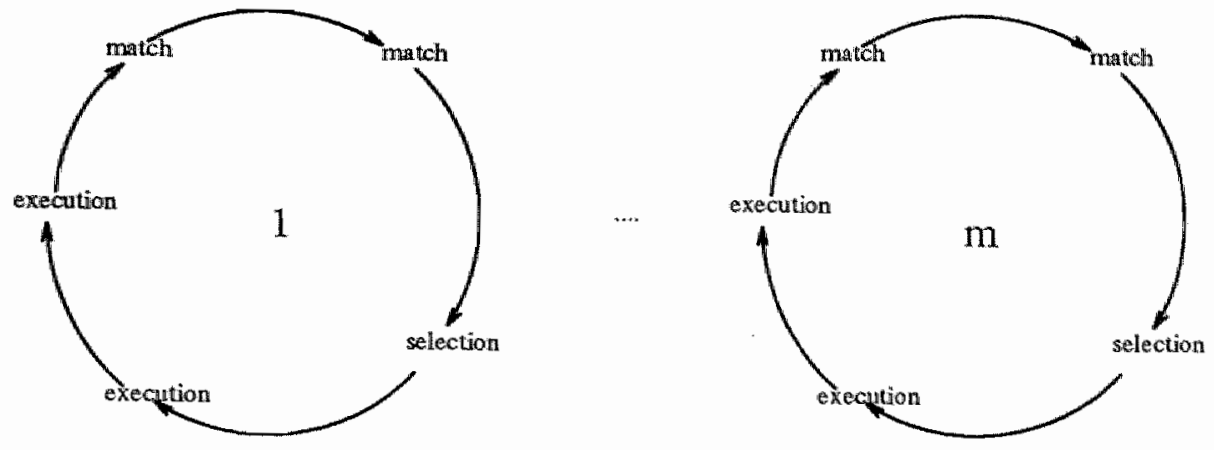

Figure 6.2: Control cycles in a distributed-control blackboard architecture.

$$
\begin{aligned}
& P L=\{\text { ready, command }\} \\
& C=\overline{\text { ready }} \cdot \text { match } h_{i_{1}} \ldots \text { match }_{i_{k}} \text { sel.stop. } \\
& \left(\overline{\text { done }}+\sum_{r=1}^{m}(\text { command }(r) . C)\right) \\
& \text { Proc }_{r}=\text { ready. } \overline{\text { command }}(r) \cdot e x_{r} \cdot P r o c_{r}, r=1, \ldots, m
\end{aligned}
$$

In the equations in Definition 14, process $C$ is a cycle performing the control cycle as in Definition 6.4 except that the execution of a KS is transferred via port command to dedicated processors. The cycle $C$ is started each time a processor Proc announces to be ready. The communication over label command passes an identifier of the task to be executed as selected by sel. An illustration is given in Figure 6.3. No synchronisation of concurrently executing KSs by different processors is implied.

Evidently, the equations in Definition 13 and 14 transform to Equation (6.11) (see Proposition 1) when $m=1$. Typically, the first set of equations define the control cycles in a distributed-control blackboard architecture, while the second set of equations define a parallel blackboard architecture. Not included in these equations is the possible distribution of the blackboard itself.

The discussion in this section presented the control units of distributed and parallel blackboard systems as modifications of a control unit in a 


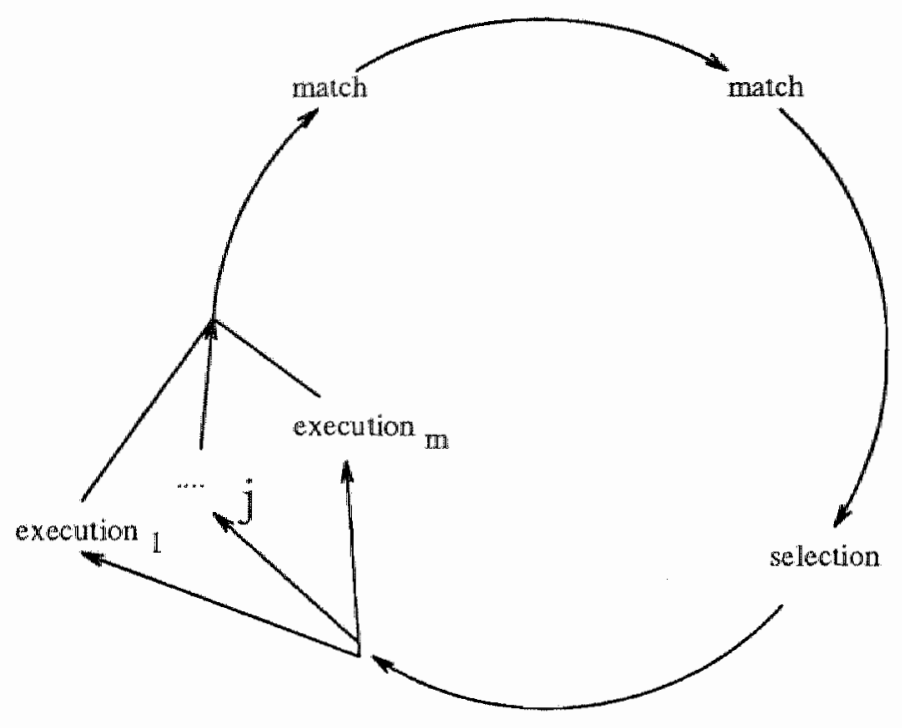

Figure 6.3: Control cycle in a parallel blackboard architecture.

sequential blackboard system (an illustration of the flow of the current discourse is provided by Figure 6.4).

Another approach to defining concurrent blackboard architectures would begin with the blackboard metaphor and look at ways of implementing this metaphor in a distributed environment. Examples of concurrent blackboard architectures resembling the blackboard metaphor closely will be encountered in Appendix A and discnssed in Chapter 7.

\subsection{Blackboard}

In the previous section we viewed the blackboard as a process communicating with its processing environment via read and write actions. However, the blackboard contains also a data structure (with the same name) which is the sink of write actions and the source for read actions. There are no real constraints on the definition of a blackboard, but it is useful in providing the blackboard with structure both for efficient access as well as for modelling purposes of the problem-solving process 

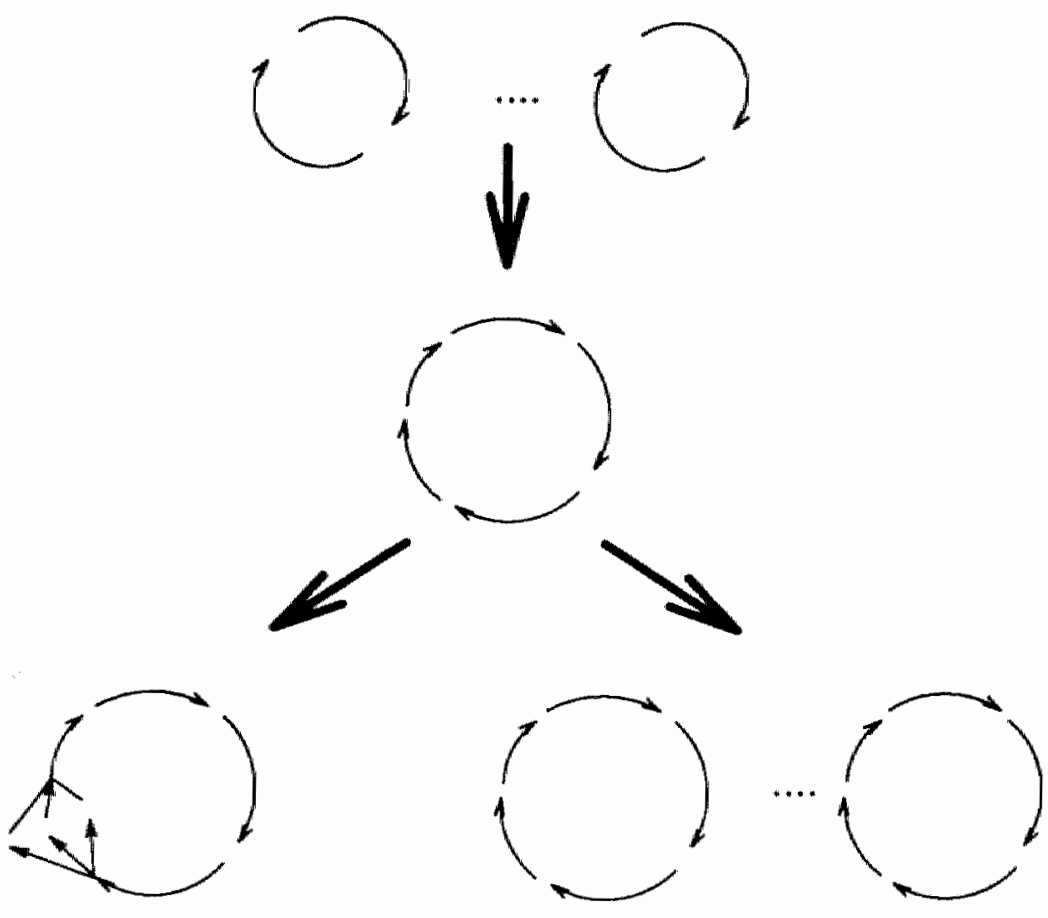

Figure 6.4: Our discussion leading finally to a specification of control units in parallel and distributed-control blackboard systems. 
implemented by the blackboard system (we note that the contents of the blackboard represents at any time the current state of the problemsolving process). Certain customs have emerged in regard of the definition of a blackboard structure. These customs should not be seen as constraints, however. This section presents customary terminology and its definitions. We use static notation to define this terminology.

In one usual approach to creating structure on the blackboard is provided by subdiving the blackboard into several panels, each subdivided into several levels (at least one). Each blackboard level may contain units with some internal structure which could in principle vary for each unit. ${ }^{4}$ Units consist of an identifier and (structured) data; they represent the most primitive named entities of a blackboard structure.

Definition 15 A blackboard unit is a data structure filled with data together with an identifier: bb-unit $=\{$ unit-id, data $\}$.

Definition 16 A blackboard level is a dynamic abstract store for blackboard units together with an identifier (the ordering of levels is represented by $<1$ ):

$$
b b-\text { level }=\left\{\text { level-id,bb-unit } t^{*}\right\}
$$

In nearly all blackboard systems, the units on a blackboard level are of the same type, but this is no necessary restriction. In fact, levels may be introduced originally to typify units.

Definition $17 \mathrm{~A}$ blackboard panel is a (possibly partially) ordered set of blackboard levels together with an identifier (the ordering of levels is represented by $<1$ ):

$$
\text { bb-panel }=\left\{\text { panel-id, bb-level }{ }^{+},<_{1}\right\}
$$

Definition 18 A blackboard is an (also possibly partially) ordered set of blackboard panels, together with a number of procedures for accessing its constituents and (optional) additional organising relations (the ordering of panels is represented by $\left.<_{p}\right)$ :

$$
B B=\left\{b b-\text { panel }^{+},<_{p}, b b-\text { prac }^{+}, \operatorname{Rel}^{*}\right\}
$$

\footnotetext{
${ }^{4}$ In the literature different names can be found for the basic elements on the blackboand. We mame "nnit', 'hypothesis", and 'object". Of them 'unit' is the most common one.
} 
The set $b b$-proc ${ }^{+}$denotes procedures for accessing the blackboard. Additional structuring of the blackboard can be achieved by defining (possibly labelled) relations between the units. These relations are denoted in the definition by $R_{e l}^{*}$. Each relation is described as follows: $R e l=\{[l a b e l], R\}, R \subseteq U \times \ldots \times U$, where $U$ is the set of all units. A (possibly labelled) binary relation between the units on the blackboard represents links between pairs of blackboard units.

Normally, links or relations are not labelled, although labelling may be quite useful. The relation denoted by a particular $R$ can be the empty set (when no relations are used currently). Further, a binary relation $R$ in $R e l$ can be both asymmetric and symmetric. An asymmetric binary relation represents an undirected graph, while a symmetric binary relation represents a directed graph. Nodes in such graphs stand for units; arcs for links. A directed graph is commonly used to model the development of the problem-solving process; hence, links are used to indicate the support of intermediate results. Together, the links constitute a dependency network. In contrast, undirected graphs are useful to represent coherency of findings and symmetric links stand for associations between them.

We would like to remark that both the number of units on a blackboard level and the number of elements in each relation $R_{e l}^{*}$ are dynamic. Usually, the subdivision of the blackboard into panels and of panels into levels is static as is the internal structure of units on a specific blackboard. In Chapter 7, some thought will be given to allowing also dynamic definition of each of these latter elements.

In several blackboard systems the different levels of blackboard panels designate abstraction levels. But one may, of course, think of other hierarchical organisations of a blackboard useful for modelling the problem domain. Some examples are modelling of geographical, physical, or functional divisions of the problem domain, as well as the often used division of a blackboard into two panels (with corresponding levels) for metaknowledge and domain knowledge.

We conclude this section with a definition for the term hierarchy of blackboard objects.

Definition 19 A hierarchy of blackboard objects is a directed, acyclic graph in which the nodes represent those abjects. 
The term 'hierarchy' is used in blackboard system descriptions to indicate whether partial orderings of blackboard objects, such as units, levels, and panels, are used.

\subsection{Knowledge sources}

Knowledge sources comprise an action part (possibly a composite of activities) and a separate data structure with data about the KS for use by the control unit.

Definition 20 A knowledge source or $\mathrm{KS}$ is an activity, implemented as a piece of program, called the KS action part, together with a data structure that describes the action part (the KS descriptor, see Definition 21): $K S=\left\{K S_{\text {descr }}, K S_{\text {action }}\right\}$.

A KS descriptor contains at least an identifier and a condition. A condition describes the blackboard states for which that KS is annotated as applicable. Optional elements of a KS descriptor are an action to be executed immediately upon the occurrence of a blackboard state satisfying the condition, a description of data to be used as input for the corresponding action part (variable binding), and additional relevant data.

Definition 21 A KS descriptor is defined as:

$K S_{\text {deser }}=\{$ identifier, condition, $[$ action $],[$ binding $],[$ data $]\}$.

In Definition 3 we presented KS instantiations as pairs, consisting of a KS and a satisfying blackboard state. Only part of the information contained within such a pair is used for activation and selection purposes. Therefore, most implementations create a special data structure to represent the relevant information from a KSI pair, cf., the "knowledge source activation record' or 'KSAR' in BBI (Hayes-Roth, 1985).

Definition 22 A knowledge source activation record or KSAR is a data structure representing relevant information for a specific KSI (relevant to activation and selection purposes). 
Definition 23 The condition of a KS defines the set of blackboard states for which this $K S$ is annotated as applicable: condition $\subseteq B S$, where $B S$ denotes the set of all possible blackboard states (for a given blackboard definition).

As indicated earlier, it is usually not feasible to monitor the complete blackboard state. For this reason, often state changes are monitored and the condition in a KS descriptor is structured in a two-fold manner: one part, the trigger, which specifies what changes have to occur before a KS becomes applicable, and the precondition specifying other requirements to be fulfilled by the actual blackboard state. Apart from these efficiency considerations, splitting a condition into parts checking either for state changes or for blackboard states can be advantageous in another sense. Some conditions are represented in a natural way using state changes, while other conditions may be represented easily using states. As all relevant aspects of the state of the problem-solving process are represented on the blackboard, the state of the problem-solving process becomes synonymous with the state of the blackboard.

Changes of the blackboard state are a consequence of KS actions. The action part of a KS is denoted by $K S_{\text {action. }}$. Since we are interested only in a formal model of blackboard architectures, we confine ourselves in the description of the action part of a KS to describing the possibilities for interaction with its enviromment. The rest of the activities inside a KS are treated as a black box. ${ }^{5}$

Interactions of KSs with their environment may be classified as:

- external interactions (man-machine interactions, communication with other subsystems in case of concurrency) and

- blackboard interactions (ports read and write in our notation for dynamic aspects, bb-proc as part of the definition of a blackboard).

\footnotetext{
${ }^{5}$ One of the more important features of blackboard architectures is that they, in principle, allow the use of different kinds of knowledge representation and different inference mechanisms within different JKSs. Thus, the tools and mechanisms prowided by a blackboard framework are very important whenever one has to choose such a framework. But in our view it is not significant for considerations concerning the archilecture.
} 
Communication with other subsystems is not considered. Man-machine interactions do not interact with other components in a blackboard architecture and can be seen as internal actions. This leaves blackboard interactions as our main concern in describing $\mathrm{KS}$ action parts in blackboard architectures. These blackboard interactions comprise read actions that can be viewed as (postponed) input for a KS (see also the definition for execution of KSs in Section 6.4) and as having no external effects (apart from blocking access to parts of the blackboard in cases of concurrent blackboard access).

Write actions, being also blackboard interactions, have immediate consequences for the problem-solving process in a blackboard architecture. Possible (elementary) write actions are:

- creation of a new unit,

- modification of an existing unit,

- deletion of an existing unit.

All of these write actions result in state changes. It depends on the particular architecture which state changes are actually recorded. For instance, some blackboard architectures do not provide the possibility of deleting any units.

Definition 24 An event is a data structure representing a state change.

Usually, an event is only an abstraction of the corresponding state change. In AGE events are just labels, called event types (Nii \& Aiello, 1979). Events in some other systems, such as GBB (Gallagher et al,, 1988), also identify the location of a change and the kind of change that has occurred. In Blondie, we created events for each modified attribute without recording information about the old or new value of the attribute.

Generally, triggers are used in the conditions of KSs to specify which state changes, or rather events, are of interest to a KS. Triggers can be described completely using conjunctions, disjunctions, and abstractions of events. Many blackboard systems, for that matter, only allow abstractions. An abstraction of an event is the set of all events that have one or more attributes in common. An example of an abstraction is a 
trigger indicating interest in the modification of a specific attribute of all units on a particular blackboard level. As the set of units on any particular blackboard level is not static, such a trigger can not be expressed a priori as a conjunction of events.

Definition 25 A trigger for a KS defines the set of events upon which, by definition, that $K S$ becomes relevant (i.e., the trigger declares when the knowledge in a KS becomes relevant solely in light of what should happen in the course of the problem-solving process).

The following proposition expresses the relation between possible triggers and events that can be generated in a blackboard system.

Proposition 2 The set of all possible triggers $T$ is the least set for which the following equations hold ( $E$ is the set of all events; $A_{E}$ the set of abstractions for an event e):

$$
e \in E \Rightarrow\{e\} \in T
$$

abstraction:

$e \in E$, abstraction $\in A_{e} \quad \Rightarrow \quad$ abstraction $\in T$

conjunction:

$t_{1}, t_{2} \in T \Rightarrow t_{1} \cap t_{2} \in T$

disjunction:

$$
t_{1}, t_{2} \in T \Rightarrow t_{1} \cup t_{2} \in T
$$

Not many current blackboard architectures allow combination of events related to state changes occurring during different cycles of the problemsolving process in triggers. If possible indeed, the definition of events is extended inherently with an identifier of the cycle on which the corresponding state change occurred, although the aforementioned rules for composition can be applied only to events generated within the same cycle. The inability to combine events from different cycles has as a consequence that an arbitrary blackboard state and history can no longer be fully specified using events. This consequence is expressed by Proposition 4 .

Definition 26 The history of a blackboard state is the sequence of blackboard states that occurred before and up to this state.

Proposition 3 Let $s_{0}, \ldots, s_{n}$ be the sequence of all states the blackboard has been in from the start, then the history of state $s_{n}$ can be described as: $\left(s_{0}, s_{1}, \ldots, s_{n}\right)$, and also as: $\left(s_{0}, \Delta\left(s_{0}, s_{1}\right), \Delta\left(s_{1}, s_{2}\right), \ldots, \Delta\left(s_{n-1}, s_{n}\right)\right)$. 
Although a sequence of states contains the same amount of information as the corresponding sequence of state changes, the latter sequence clearly involves less representational data. ${ }^{6}$

Proposition 4 Let $s_{n}$ be a state with history $H\left(s_{n}\right)=\left(s_{0}, \Delta\left(s_{0}, s_{1}\right)\right.$, $\left.\Delta\left(s_{1}, s_{2}\right), \ldots, \Delta\left(s_{n-1}, s_{n}\right)\right)$, then $s_{n}$ is described completely by $H\left(s_{n}\right)$.

The history $H\left(s_{n}\right)$ contains more information than the blackboard state $s_{n}$ alone, as the history also contains information about changes whose induced results have been changed themselves.

In practice, however, it is not state changes that are used but rather abstractions in the form of events. Furthermore, a complete history of blackboard states is normally not retained. Therefore, the duality between blackboard states and state changes is lost and a triggering mechanism must be augmented with an extra check on the blackboard state in order to capture a useful KS condition. This extra check is called the precondition.

Definition $27 A$ precondition is that part of the condition of a KS that determines applicability of a blackboard by using solely blackboard state information.

We note that the write actions as used in this section are related only to blackboard units. However, links could be treated similarly as has been done, e.g., in GBB (Gallagher et al., 1988). As long as links are implemented as attributes of units, modifications of links are merely modifications of blackboard units. But, when links are represented separately, using these links for triggering and monitoring state changes may provide a quite meaningful extension.

The conditions for KSs and the way conditions are checked with blackboard states or state changes are central to continuation of the problemsolving process: when no states or events occur that generate applicable new activities, the problem-solving process would soon come to a halt. It is therefore interesting to spend some more effort in describing this

\footnotetext{
"The use of state changes involves less data, becanse it is understood implicitly that data not mentioned in a state change remains unchanged. This is a notion formalised by circumscription (Perlis, 1987).
} 
mechanism. We do this by concentrating on the 'life cycle' of a KS instantiation and variations thereof.

We first define the states a $\mathrm{KST}^{7}$ could be in.

Definition 28 The distinct states a KSI can be in are defined as follows:

1. A KSI is called triggered, when its trigger has been satisfied.

2. A KSI is called applicable, when its trigger and precondition have been satisfied.

3. A KSI is called selected, when a decision has been made to execute this KSI.

4. After a KSI has been executed, the KSI is called executed.

5. When a KST has been interrupted during execution (either by itself, by other components within the blackboard system, or from the outside), the KSI is called suspended.

6. When information about a KSI is no longer available to the system, the KSI is called removed.

7. In all other cases a KSI is just called present.

We note that we do not describe a situation where a KSI has satisfied its precondition, but not its trigger. As a trigger is mostly a preprocessing mechanism to increase efficiency related with checking blackboard states, we have reason to assume that reversal in the order of trigger and precondition does not occur.

Based on these states, we now consider which state transitions are possible and what meaning can be given to any transition. In this description of state transitions, no distinction is made between the triggered and applicable states, as essentially they only represent a subdivision of the satisfaction of the condition of a KS as a whole. For each state the transitions to other (directly reachable) states are given as follows:

\footnotetext{
${ }^{7}$ It depends on a particular blackboard architecure whether there is a distinction being made between a KS and an instantiation of that KS. We use the term KSI also to refer to a KS when no distinction is made between a KS and instantiations thereof.
} 


\section{triggered/applicable}

selected A usual transition where an applicable KSI is selected for execution at one time or other.

executed This transition occurs when selection is eliminated.

suspended Identical with the previous transition, except that execution has been interrupted.

removed This transition is sometimes used when an applicable KSI becomes no longer applicable and needs therefore to be removed from the system.

selected

triggered/applicable This transition occurs when, upon selection, it appears that a KSI is no longer applicable, but remains in the system (e.g., in a triggered state). This procedure is part of the BB1 control cycle.

executed This is a usual transition.

suspended This transition occurs only when a KSI is suspended during immediate execution upon selection.

removed This transition can occur, e.g., when a number of KSIs are selected for execution, but other actions make execution superfluous (e.g., when a solution has been found).

executed

removed This transition is quite straightforward.

present This transition can be observed in a blackboard architecture closely resembling the blackboard metaphor, i.e., where KSs can be seen as agents performing a cycle of monitoring the blackboard and performing some activities when their condition is met. After execution, KSs continue by monitoring the blackboard, thus returning to a present state.

suspended

triggered/applicable This transition denotes a situation where a suspended KSI is treated as an applicable activity once more. 
selected This transition can be observed when a KSI is selected automatically again after suspension.

executed This transition coincides with treating a KSI as being executed after interruption (discarding the not executed part of the KSI).

removed The transition in which a suspended activity is removed from the system.

present This transition occurs when, after interruption, a KSI is treated as just present.

\section{present}

triggered/applicable This transition will occur when a trigger and/or precondition is matched as described in the previous sections.

selected This transition is only meaningful when selection is automatic after the condition is satisfied, but execution can be postponed for some (short) time. This transition is used, e.g., in systems where all applicable KSIs are selected immediately for execution one after the other.

executed In this transition, a KSI goes immediately from being present to being executed, coinciding with a demon-like implementation.

suspended This transition is similar the previous one, with the exception that execution of the KSI was suspended.

Definition 29 The life cycle of a KSI in a given blackboard architecture is described by the possible states and state transitions for that KSI.

Based on the observations of possible or allowed states and state transitions, a dynamic representation of the most complex imaginalyle life cycle ( $L C$ ) of a $\mathrm{KSI}$ could be represented as follows:

$$
\begin{aligned}
L C & =p \cdot P \\
P & =c \cdot C+s \cdot S+e \cdot(E+i . I) \\
C & =s \cdot S+e \cdot(E+i . I)+r \cdot \overline{\text { done }} \\
S & =c \cdot C+e \cdot(E+i . I)+r . \overline{\text { done }} \\
E & =p \cdot P+r \cdot \overline{\text { done }} \\
I & =p \cdot P+c . C+s \cdot S+e .(E+i . I)+r . \overline{\text { done }}
\end{aligned}
$$


The lowercase symbols in these equations correspond to actions in the following way: $p$ is becoming present; $c$ is satisfying the condition; $s$ is being selected; $e$ is being executed; $i$ is being interrupted (suspended); and $r$ means being removed.

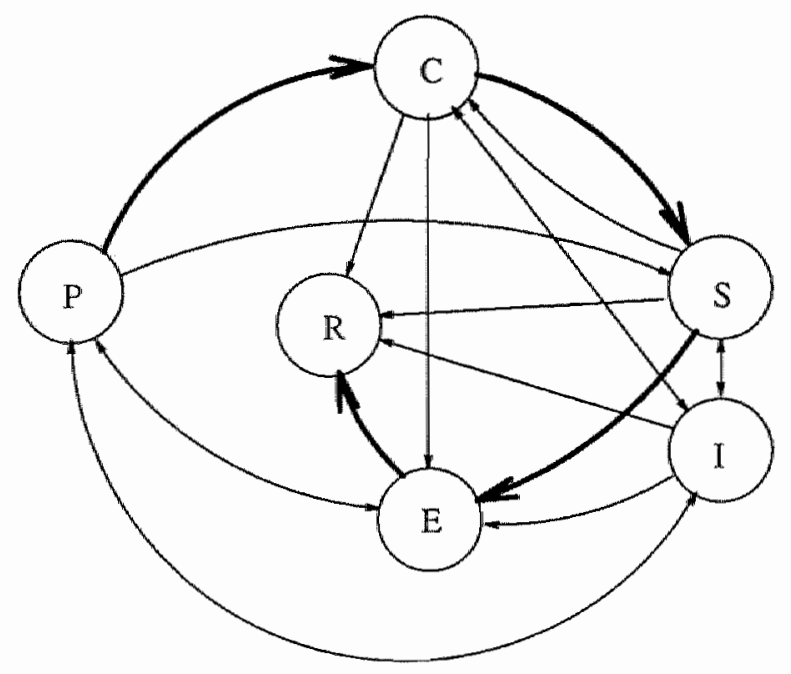

Figure 6.5: States and transitions for a IXS instantiation. $P=$ present; $C=$ applicable (condition satisfied); $S=$ selected; $I=$ suspended (interrupted); $E=$ executed; $R=$ Rerroved.

The possible transitions and states are shown in Figure 6.5. Existing blackboard architectures hardly allow such complexity in the life cycles of their KSs. For one thing, most blackboard architectures do not allow interruption of a KS which is being executed. Actually, a quite common life cycle would be represented by just the following equation:

$$
L C^{\prime}=\text { c.s.e.r. } \overline{\text { done }}
$$

(in this example a KSI becomes present if and only if (part of) its condition is satisfied). This common life cycle is shown in Figure 6.5 by the bold arrows. At least, a life cycle should contain the actions $c$ and $e$ (in fact, a minimal life cycle can be found in some architectures, see the descriptions of, e.g., POLIGON and AGORA in Appendix A). 


\subsection{Related work}

We now discuss other work related to our formalisation of the blackboard architecture. The work that is most closely related to ours was recently published by Craig in (Craig, 1991). In this book, Craig presents formal specifications of a generic version of the blackboard architecture and of his CASSANDRA architecture (Craig, 1989). These specifications are written in the specification language Z (Spivey, 1988; Spivey, 1989), which is a model-oriented formalism, while our language is based on the algebraic formalism CCS. A distinction between the two formalisms is that $\mathrm{Z}$ is used primarily to describe sequential systems, while CCS describes concurrent systems.

The main difference between our work and Craig's work is a distinction between breadth versus depth. Craig specifies only two architectures, but presents more detail than we did in our studies. This enables Craig to go farther in deriving properties of the described architectures. On the other hand, Craig assumes a generic version of the blackboard architecture, whereas part of our efforts were directed at defining what constitutes such a generic version. For this, we chose to study a broad array of existing architectures. Furthermore, we wanted to investigate the possible variation (both existing and imaginable) for blackboard architectures. This also lead us to study a larger number of blackboard architectures, although we acknowledge that this was at the expense of a more detailed and formal analysis of properties of the examined architectures. In view of these observations, we feel that Craig's work and ours is complementary.

Other formalisation efforts in the area of blackboard systems have concentrated mainly on control issues. Most notably, we mention and briefly describe work by Bachimont (1991a; 1991b) and Silverman (1989). Our work is related also to formalisation of other architectures, such as Wand's proposal for a formal model of objects (Wand, 1988). We conclude this section by mentioning some work on formal modelling languages in which characteristics from the blackboard architecture have been adopted (Kelemen, 1991; Dassow, 1991).

Bachimont (1991a; 1991b) has developed a logical framework for blackboard control. His approach concentrates on two issues in control. The first issue, called coherence, is concerned with handling ambiguity and 
conflicts between hypotheses on the blackboard generated by the KSs. The second issue, called convergence, deals with the selection of activities in order to direct problem solving towards a solution. Bachimont has developed a logic and a truth-maintenance system for this logic to achieve coherence. Convergence is dealt with by the development of a dynamic logic which provides a means for formally describing control activities. A theorem prover for the dynamic logic can serve as the control unit for a blackboard system.

Silverman (1989) describes a first step towards modelling the selection (for invocation) of $\mathrm{KSs}$ by treating it as a decision theoretic problem. The author assumes several processors for executing tasks. (A sequential blackboard system can be seen as a special case.) The (logical) processors need not have access to the same information. Also, processors may have different expectations regarding the results of the activities. The approach makes use of a cost function representing the difference between an actual state and the desired end state (goal). Selecting the best activities for the processors corresponds to minimising the cost function for the expected next state.

These two approaches by Bachimont and Silverman as well as our formalisation are directed specifically at describing aspects in blackboard systems. Kelemen (1991) and Dassow (1991) present languages for formally describing distributed, cooperative systems, not just blackboard systems (see also page 29). Kelemen and Dassow propose languages (grammar systems) that adopt characteristics of the blackboard architecture for specifying cooperative interaction, most notably the notion of using a 'blackboard' for storing and communicating hypotheses. Thus, these languages are aimed at describing a larger group of systems. The languages proposed by Kelemen and Dassow concentrate on modelling the cooperative behaviour of distributed systems, whereas we were more interested in describing the architecture of blackboard systems. Thus, the two approaches can be seen as complementary.

\subsection{Evaluation}

In this chapter we established a formalisation of the blackboard architecture. Our formalisation has been used to describe a collection of existing blackboard architectures (see Appendix A). The more formal 
approach to describing existing architectures as presented in this chapter facilitated comparison of these architectures and analysis of their features and characteristics. Our formalisation efforts have also aided us in obtaining a clearer understanding of both the described blackboard architectures and the blackboard architecture in general. The results of our analyses of existing blackboard architectures are given in the next chapter.

On a general note, the following observations can be made. In several publications on blackboard systems, the descriptions appeared to be incomplete and sometimes even inconsistent. Yet, incompleteness is in a sense quite natural, as papers and reports typically tend to focus on particular features and characteristics and leave others undiscussed. Furthermore, inconsistencies could be explained possibly as having occurred in different versions of the same system or as originating from discrepancies between design and implementation. Nevertheless, both incompleteness and inconsistency hinder replication of research results and make arriving at a general theory of blackboard architectures unnecessarily difficult.

A number of relevant aspects of blackboard architectures can not be expressed by our formalisation. These aspects are:

Causality With our current formalisation it is impossible to express directly how (internal) choices within a described system depend on previous decisions or available information. Causality occurs within blackboard systems most notably in determining applicability of KSs and in evaluating a stop criterion. 'This is inherent in the chosen basis for our formalisation, i.e., CCS. The description language CCS is more suitable for describing (observable) behaviour, than for specifying how a system should behave in attending circumstances.

Parametrisation On some occasions we found that our formalisation lacked a suitable way of using parameters. This became especially apparent in trying to describe the locking mechanism of the blackboard in the parallel blackboard shell Blondie-II. CCS (and thus our formalisation) does not provide an appropriate mechanism for identifying distinct regions on the blackboard due to the lack of facilities for using parameters, thus complicating specification of 
blackboard interactions which take into account locks on identified regions.

As a last remark, we have not used our formalisation to try to derive formally equality of different implementations or to 'prove' disjunction between the blackboard framework and other computational frameworks. The next chapter, however, addresses the issue of discriminating between the blackboard architecture and other frameworks based on the analyses of our formal descriptions. 


\section{Chapter 7}

\section{Options and Variations}

This chapter deals with the answers to the central questions posed in Chapter 1 . We have applied our formalisation in Chapter 6 to describe a number of existing blackboard architectures. Apart from the three versions of Blondie, eleven more blackboard architectures and blackboardlike architectures were chosen (see Table 7.1 for a list of these architectures). We note that some of the blackboard arclitectures are used as blackboard frameworks, while others occur as blackboard applications. Typically, blackboard frameworks allow more variation of their components than blackboard applications, where for most aspects a choice has been made for a specific option. In our discussion mainly the architectural aspects are treated and we abstract from the applications involved.

Appendix A contains the actual formal descriptions of the selected blackboard architectures. We studied the descriptions of these blackboard alchitectures to determine which options and variations with in the blackboard model have been used. Each variable aspect could be seen as a dimension. The dimensions together define a space. A specific blackboard architecture could be placed in this space by examining which options have been selected for that architecture. By placing existing blackboard architectures in the space defined by the dimensions, we would create a map of existing blackboard architectures. Examination of this map would reveal options and combinations of options within blackboard architectures that have been and have not been implemented.

Figure 7.1 presents an example of such a map, where only two (hypothet- 


\begin{tabular}{|c|c|}
\hline system & description \& essential reference \\
\hline Hearsay-II & $\begin{array}{l}\text { the first, and therefore perhaps prototypical, implemen- } \\
\text { tation of a blackboard system (Erman et al., 1980) }\end{array}$ \\
\hline Hearsay-III & $\begin{array}{l}\text { an abstraction of Hearsay-II with some additional } \\
\text { interesting features (Erman et al., 1981) }\end{array}$ \\
\hline$\overline{D V M T}$ & $\begin{array}{l}\text { a distributed blackboard system which has been used } \\
\text { mainly as a research tool in the field of Distributed AI, } \\
\text { though many of the thus inspired enhancements seem } \\
\text { useful for blackboard architectures in general } \\
\text { (Lesser \& Corkill, 1983) }\end{array}$ \\
\hline HASP/SIAP & $\begin{array}{l}\text { represents a different kind of application (a monitoring } \\
\text { task) from Hearsay-II (a pattern recognition application): } \\
\text { it had interesting consequences for its design } \\
\text { (Nii et al. 1988) }\end{array}$ \\
\hline $\mathrm{AGE}$ & $\begin{array}{l}\text { a blackboard framework which betrays in some aspects } \\
\text { a relation to IASP/SIAP (Nii \& Aiello, 1979) }\end{array}$ \\
\hline $\mathrm{CAGE}^{-}$ & $\begin{array}{l}\text { a blackboard framework, which, based on } \mathrm{AGE} \text {, allows to } \\
\text { specify opportunities for concurrency (Aiello, 1986) }\end{array}$ \\
\hline POLIGON & $\begin{array}{l}\text { a blackboard framework according to its developer, but } \\
\text { deceptively different because of extensive exploitation } \\
\text { of opportunities for concurrency (Rice \& Aiello, 1989) }\end{array}$ \\
\hline$\overline{\mathrm{B}} \overline{\mathrm{B}} 1$ & $\begin{array}{l}\text { a blackboard framework, which has set a standard for } \\
\text { sophistication of control (Hayes-Roth, 1985) }\end{array}$ \\
\hline AGORA & $\begin{array}{l}\text { perhaps not really a blackboard framework, but with } \\
\text { some similarities; especially interesting as it is the } \\
\text { successor to (and presumably improvement upon) } \\
\text { Hearsay-II as an environment for speech recognition } \\
\text { research (Bisiani et al., 1987) }\end{array}$ \\
\hline$\widehat{\mathrm{GBB}}$ & $\begin{array}{l}\text { a carefully designed and efficient blackboard framework, } \\
\text { attempting to be generic, hence the name Generic } \\
\text { BlackBoard shell (Corkill et al., 1986) }\end{array}$ \\
\hline CASSANDRA & $\begin{array}{l}\text { a distributed blackboard framework designed with an } \\
\text { emphasis on nodularity (Craig, 1989) }\end{array}$ \\
\hline
\end{tabular}

Table 7.1: Overview of formally described blackboard architectures (apart from Blondie, Blondie-II, and Blondie-III). 
ical) dimensions are used. On this map a specific blackboard application typically is represented by just a dot (e.g., Hearsay-II). A blackboard framework stretches out over several possible options in multiple dimensions and is represented therefore as a set. The outer boundary in Figure 7.1 represents the blackboard model. This boundary is specifically not a rectangle to indicate that the decision whether a specific architecture is in fact a blackboard architecture can not be determined by examining each dimension separately: interactions may exist. A generic blackboard framework, such as GBB (Corkill et al, 1986), is indeed truly generic when it would be represented on the map of blackboard architectures by a set including all representations of possible blackboard architectures.

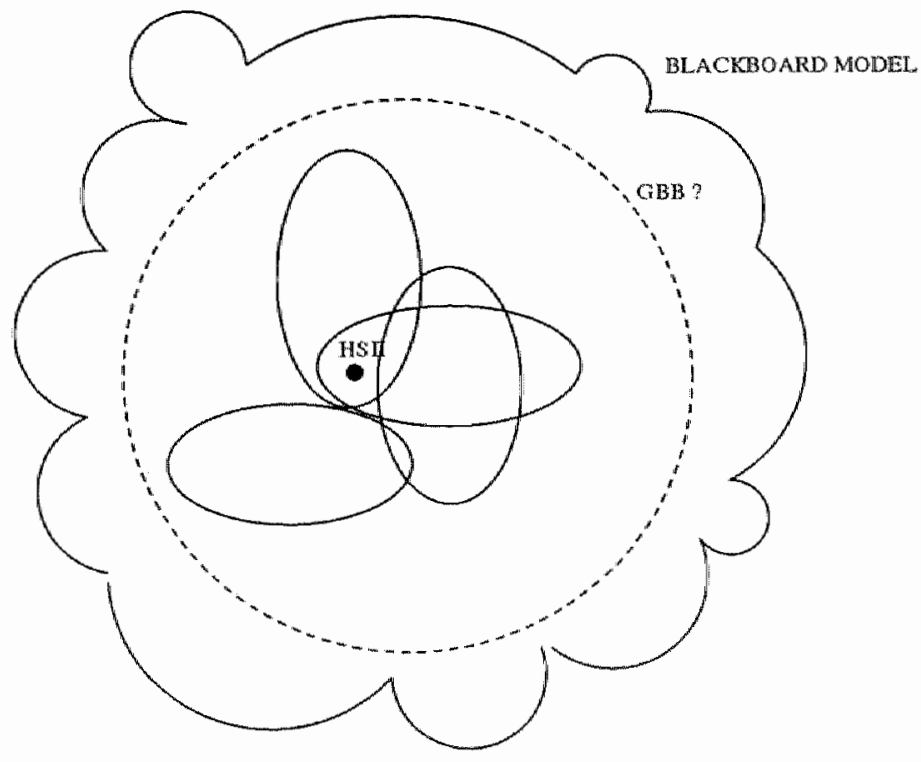

Figure 7.1: A map of existing options and variations.

Evidently, the list of examined blackboard architectures is not complete: many blackboard architectures have not been considered and many of the possibly interesting features of such non-selected architectures were i. hus excluded from the analysis. A complete analysis of all existing blackboard architectures is obviously out of the question: too many already exist and are documented in a wide range of publications, while 
still others are being implemented and documented regularly. However, the selected blackboard architectures are all rellatively well-known and therefore documentation for these systems is easily accessible. In effect, these architectures serve as reference points to many other implementations. It is thus that we view the selected list of blackboard architectures as a useful and large basis for deriving an overview of options and variations in existing blackboard architectures. This overview is presented in Section 7.1.

After our overview of options and variations in existing blackboard architectures, it becomes easier to see where observed options and combinations of options can be extended and supplemented. Ideally, such an effort would lead eventually to an overview of the complete range of options and possibilities in blackboard architectures. Section 7.2 attempts to give such an overview and gives semantic interpretations for the described options. As an overview of the complete range of options includes already implemented options, Section 7.2 repeats portions of Section 7.1. However, as these two sections both address a different question, we have decided in favour of maintaining this inherent duplication. Section 7.3 addresses the issue of what minimally constitutes a blackboard architecture and what features and belhavioural characteristics distinguish blackboard architectures from other kinds of frameworks.

Materiall from this chapter has been published previously in (Velthuijsen \& Braspenning, 1991a; Velthuijsen \& Braspenning, 1991b).

\subsection{Existing options and variations}

Tables $7.2,7.3,7.4$, and 7.5 give an overview of options derived from the formal descriptions of existing blackboard architectures given in Appendix A. The presentation is further condensed in Table 7.6, which serves as a guideline in the description of options and variations in existing blackboard architectures. The symbols used in these tables have the following meaning. The symbol "+" indicates that this option is present in the corresponding architecture; "-." denotes that this option is not present in the corresponding architecture. The symbol " $\square$ " denotes that this option is variable (user-definable). The terms in these tables are keywords coinciding with the original terms used in the respective publications (see also Appendix A). In some cases we could not 
derive from available publications which particular option was selected for a certain architecture. This is indicated by "?" in the tables. We refer the reader to the tables presented here or to Appendix $A$ for details not included in the following discussion. Section 7.2 discusses semantic consequences of options and variations. To avoid repetition of the same arguments this section largely refrains from discussing semantics.

\subsubsection{Blackboard}

We now present variations of blackboard defimitions and interactions observed in existing blackboard architectures. We also show observed variation of events.

\section{Organisation}

Basically, two different organisations of a blackboard are used. In early blackboard systems the blackboard was subdivided into different levels or ordered sets of levels (termed panels). In some later implementations this kind of organisation was augmented towards further specialisation in the form of classes in a class hierarchy.

Class hierarchy. Some blackboard systems use classes and a class hierarchy for structuring and organising the contents of the blackboard. A class hierarchy allows storage of blackboard entities in more specialised regions than possible with the use of blackboard levels and blackboard panels, which are more common.

Panels. The observed variation ranges over blackboard systems with no panels (in cases where a class hierarchy is used), with only one panel, with two or three panels, and with a variable number of panels. In only one case (DVMT) a hierarchical relation (abstraction hierarchy) between the different panels could be found.

Levels. The number of levels is sometimes fixed (when a specific application was considered). Blackboard applications use mostly a (partial) ordering of levels. When a blackboard architecture uses classes, some form of (class-)hierarchy is obligatory. Blackboard frameworks that do not use a class hierarcliy do not predefine any other hierarchy either. 


\begin{tabular}{|c|c|c|c|}
\hline & Hearsay-II & Hearsay-III & DVMT \\
\hline $\begin{array}{l}\text { class-hierarchy } \\
\text { panels } \\
\text { - hierarchy } \\
\text { levels } \\
\text { - hierarchy }\end{array}$ & $\begin{array}{c}\overline{-} \\
- \\
\text { ordered }\end{array}$ & $\begin{array}{l}t \\
2 \\
- \\
\square\end{array}$ & $\begin{array}{c}\overline{3} \\
\text { part. abstraction } \\
\text { part. ordered }\end{array}$ \\
\hline $\begin{array}{l}\text { units } \\
\text { - attr-value pairs } \\
\text { - typed } \\
\text { - hierarchy }\end{array}$ & $\begin{array}{l}\text { hypotheses } \\
+ \\
\text { time orclered }\end{array}$ & $\begin{array}{c}\text { units } \\
+ \\
+\end{array}$ & $\begin{array}{c}\text { hypotheses } \\
+ \\
\square\end{array}$ \\
\hline $\begin{array}{l}\text { Tinks } \\
\text { - directed } \\
\text { - labelled }\end{array}$ & $\begin{array}{l}+ \\
+\end{array}$ & $\begin{array}{l}t \\
t \\
t\end{array}$ & $?$ \\
\hline $\begin{array}{l}\text { events } \\
\text { - structure : } \\
\text { - abstraction } \\
\text { - units } \\
\text { - links } \\
\end{array}$ & $\begin{array}{c}\text { change sets } \\
\text { attr.-value } \\
\text { pairs } \\
+ \\
+ \\
+\end{array}$ & $\begin{array}{c}\text { changed APM } \\
\text { templates } \\
\text { - } \\
- \\
+ \\
?\end{array}$ & $\begin{array}{l}? \\
+ \\
+ \\
?\end{array}$ \\
\hline $\begin{array}{l}\text { Interactions } \\
\text { - read } \\
\text { - create } \\
\text { - modify } \\
\text { - delete } \\
\end{array}$ & $\begin{array}{l}+ \\
+ \\
+ \\
+\end{array}$ & $\begin{array}{l}+ \\
+ \\
+ \\
+\end{array}$ & $\begin{array}{l}+4 \\
+ \\
+ \\
- \\
-\end{array}$ \\
\hline $\begin{array}{l}\text { description } \\
\text { - binding } \\
\text { - features }\end{array}$ & $\begin{array}{c}\text { stimulus } \\
\text { frame } \\
?\end{array}$ & $\begin{array}{c}\text { immediate } \\
\text { code } \\
\square\end{array}$ & $?$ \\
\hline $\begin{array}{l}\text { Condition } \\
\text { - trigger } \\
\text { - precondition }\end{array}$ & + & + & $\begin{array}{c}\text { goal } \\
+\end{array}$ \\
\hline $\begin{array}{l}\text { action part } \\
\text { - code } \\
\text { - rules } \\
\text { - evaluation } \\
\text { - interruptible }\end{array}$ & $\begin{array}{l}+ \\
- \\
-\end{array}$ & $\begin{array}{l}+ \\
- \\
-\end{array}$ & $\stackrel{+}{-}$ \\
\hline $\begin{array}{l}\text { instantiations } \\
\text { - amount } \\
\text { - description }\end{array}$ & $\begin{array}{l}\infty \\
\text { resp. frame } \\
\text { stim. frame }\end{array}$ & $\begin{array}{c}\infty \\
\text { unit on } \\
\text { sched. BB }\end{array}$ & $\begin{array}{c}\infty \\
\text { resp. frame }\end{array}$ \\
\hline $\begin{array}{l}\text { Control cycle } \\
\text { KS cycle } \\
\text { concurrency } \\
\text { - parallelism } \\
\text { - distributed }\end{array}$ & $\begin{array}{l}+ \\
+ \\
- \\
- \\
-\end{array}$ & $\begin{array}{l}+ \\
- \\
- \\
- \\
-\end{array}$ & $\begin{array}{l}t \\
+ \\
+ \\
+ \\
H\end{array}$ \\
\hline $\begin{array}{l}\text { matching } \\
\text { - event-based } \\
\text { - KS-based } \\
\text { numount } \\
\text { number of activities }\end{array}$ & $\frac{+}{a}$ & $\frac{ \pm}{a}$ & $\frac{\hbar}{\text { all }}$ \\
\hline $\begin{array}{l}\text { selection } \\
\text { - blackboard-based } \\
\text { - KS-based }\end{array}$ & + & $\begin{array}{l}\square \\
\square\end{array}$ & + \\
\hline stop-criterion & Stop KS & - & $?$ \\
\hline
\end{tabular}

Table 7.2: Variation observed in Hearsay-II, Hearsay-III, and DVMT. 


\begin{tabular}{|c|c|c|c|c|}
\hline & HASP/SLAP & $\overline{\mathrm{AGE}}$ & CAGE & POLIGON \\
\hline $\begin{array}{l}\text { class-hierarchy } \\
\text { panels } \\
\text { - hierarchy } \\
\text { levels } \\
\text { - hierarchy }\end{array}$ & $\begin{array}{c}\overline{\overline{1}} \\
\overline{\overline{5}} \\
\text { ordering }\end{array}$ & $\begin{array}{l}- \\
>0 \\
>0\end{array}$ & $\begin{array}{l} \pm \\
\pm \\
- \\
>0 \\
\text { class }\end{array}$ & $\begin{array}{c}+ \\
+ \\
>0 \\
\text { class }\end{array}$ \\
\hline $\begin{array}{l}\text { units } \\
\text { - attr-value pairs } \\
\text { - typed } \\
\text { - hierarchy }\end{array}$ & $\begin{array}{c}\text { hyp. elements } \\
+ \\
-\end{array}$ & $\begin{array}{c}\begin{array}{c}\text { hyp. elements } \\
+ \\
- \\
\end{array} \\
\end{array}$ & $\begin{array}{c}\text { nodes } \\
+ \\
- \\
\end{array}$ & $\begin{array}{c}\text { nodes } \\
+ \\
- \\
\end{array}$ \\
\hline $\begin{array}{l}\text { links } \\
\text { - directed } \\
\text { - labelled }\end{array}$ & $\begin{array}{c}+ \\
+ \\
\text { reduct./expect. }\end{array}$ & $\begin{array}{c}t \\
t \\
\text { reduct./expect. }\end{array}$ & $\begin{array}{l}\text { atiributes } \\
+ \\
+\end{array}$ & $\begin{array}{c}\text { nodes } \\
\square \\
+ \\
\end{array}$ \\
\hline $\begin{array}{l}\text { events } \\
\text { - structure } \\
\text { - abstraction } \\
\text { - units } \\
\text { - links }\end{array}$ & $\begin{array}{c}\text { clock/problem/ } \\
\text { normal event } \\
\text { time/event }+\mathrm{KS} / \\
\text { description } \\
-/+/+ \\
+ \\
-\end{array}$ & $\begin{array}{c}\text { label } \\
- \\
+ \\
+ \\
-\end{array}$ & $\begin{array}{l}\text { taken } \\
- \\
+ \\
+\end{array}$ & $\begin{array}{c}\text { demons } \\
- \\
+ \\
+ \\
+\end{array}$ \\
\hline $\begin{array}{l}\text { interactions } \\
\text { - read } \\
\text { - creabe } \\
\text { - modify } \\
\text { - delete } \\
\end{array}$ & $\begin{array}{l}+ \\
+ \\
+ \\
+\end{array}$ & $\begin{array}{l}+ \\
+ \\
+ \\
-\end{array}$ & $\begin{array}{l}+ \\
+ \\
+ \\
- \\
\end{array}$ & $\begin{array}{l}+ \\
+ \\
+ \\
- \\
\end{array}$ \\
\hline $\begin{array}{l}\text { description } \\
\text { - binding } \\
\text { - features }\end{array}$ & $?$ & $\begin{array}{l}\text { LHSs } \\
\text { lewels } \\
\text { links } \\
\text { rule eval. }\end{array}$ & $\begin{array}{l}\text { LHSs } \\
\text { levels } \\
\text { links } \\
\text { rule eval. }\end{array}$ & $\begin{array}{l}- \\
- \\
-\end{array}$ \\
\hline $\begin{array}{l}\text { condition } \\
\text { - trigger } \\
\text { - precondition } \\
\end{array}$ & list of events & $\begin{array}{c}\text { list of events } \\
- \\
\end{array}$ & list of tokens & demons \\
\hline $\begin{array}{l}\text { action part } \\
\text { - code } \\
\text { - rules } \\
\text { - evaluation } \\
\text { - interruptible }\end{array}$ & $\begin{array}{l}+ \\
+ \\
? \\
- \\
\end{array}$ & $\begin{array}{c}+ \\
\text { variable } \\
\end{array}$ & $\begin{array}{c}\text { + } \\
\text { variable/paral. } \\
\end{array}$ & $\stackrel{+}{+}$ \\
\hline $\begin{array}{l}\text { instantiations } \\
\text { - amount } \\
\text { - description } \\
\end{array}$ & 1 & $\mathbb{1}$ & $\begin{array}{l}1 \\
- \\
\end{array}$ & - \\
\hline $\begin{array}{l}\text { controil cycle } \\
\text { KS cycle } \\
\text { concurrency } \\
\text { - parallelism } \\
\text { - distributed. }\end{array}$ & $\begin{array}{l}+ \\
- \\
-\end{array}$ & $\begin{array}{l}\overline{1} \\
\text { w. } \\
- \\
-\end{array}$ & $\begin{array}{c}+ \\
\text { opt. } \\
+ \\
+\end{array}$ & $\begin{array}{l}+ \\
+ \\
+ \\
+\end{array}$ \\
\hline $\begin{array}{l}\text { matching } \\
\text { - event-based } \\
\text { - KS-based } \\
\text { - arnount } \\
\text { number of aclivities }\end{array}$ & $\begin{array}{c}+ \\
- \\
\text { all clock } \\
\text { all problems } \\
\text { focussed events } \\
\text { warable }\end{array}$ & $\frac{ \pm}{1}$ & $\begin{array}{c}t \\
\text { opt. } \\
1-\text { all } \\
\text { variable }\end{array}$ & $\frac{t}{\text { all }}$ \\
\hline $\begin{array}{l}\text { selection } \\
\text { - blachiboard-based } \\
\text { - KS Bubased }\end{array}$ & \pm & \pm & \pm & $\cdots$ \\
\hline stop-criterion & $\begin{array}{l}\text { empty } \\
\text { event-list. }\end{array}$ & $\begin{array}{l}\text { cond. } \\
\text { on bb }\end{array}$ & $\begin{array}{l}\text { cond. } \\
\text { on bb. }\end{array}$ & - \\
\hline
\end{tabular}

Table 7.3: Variation observed in HASP/SIAP, AGE, CAGE, and POLIGON. 


\begin{tabular}{|c|c|c|c|}
\hline & $\mathrm{BBI}$ & AGORA & $\mathrm{GBB}$ \\
\hline $\begin{array}{l}\text { class-hierarchy } \\
\text { parels } \\
\text { - hierarchy } \\
\text { levels } \\
\text { hierarchy }\end{array}$ & $\begin{array}{l}- \\
2 \\
- \\
\square \\
\square\end{array}$ & $\bar{\square}$ & $\begin{array}{l}+ \\
- \\
\square \\
\square\end{array}$ \\
\hline $\begin{array}{l}\text { urits } \\
\text { - attr-wallue pairs } \\
\text { - typud } \\
\text { - hinarating }\end{array}$ & $\begin{array}{c}\text { units } \\
t \\
-\end{array}$ & $\begin{array}{c}\text { elements } \\
+ \\
\square\end{array}$ & $\begin{array}{c}\text { units } \\
+ \\
\text { ondered }\end{array}$ \\
\hline $\begin{array}{l}\text { Tinks } \\
\text { - dinected } \\
\text { - lathelled }\end{array}$ & - & $\overline{-}$ & $\begin{array}{c}\text { slots } \\
\bar{t} \\
\end{array}$ \\
\hline $\begin{array}{l}\text { events } \\
\text { - structure } \\
\text { - abstractom } \\
\text { - units } \\
\text { - links }\end{array}$ & $\begin{array}{c}\text { Iecord } \\
\text { lypet-unit-id } \\
+ \\
+ \\
\square\end{array}$ & $\begin{array}{c}\operatorname{arrival} \\
- \\
+ \\
+ \\
-\end{array}$ & $\begin{array}{c}\text { attr-val. pairs } \\
\square \\
+ \\
+\end{array}$ \\
\hline $\begin{array}{l}\text { interactions } \\
\text { - read } \\
\text { - create } \\
\text { - modify } \\
\text { - dalebe } \\
\end{array}$ & $\begin{array}{l}+ \\
+ \\
+ \\
+\end{array}$ & $\begin{array}{l}+ \\
4 \\
- \\
-\end{array}$ & $\begin{array}{l}t \\
t \\
t \\
t\end{array}$ \\
\hline $\begin{array}{l}\text { Tescription } \\
\text { - binding } \\
\text { - features }\end{array}$ & $\begin{array}{c}\text { prec., cond, } \\
\text { sched. values } \\
\text { descr. cond. vars } \\
\text { features }\end{array}$ & - & $\square$ \\
\hline $\begin{array}{l}\text { condition } \\
\text { - trigger } \\
\text { - precondition }\end{array}$ & $\begin{array}{l}\text { event eval. } \\
\text { comb. pred, }\end{array}$ & + & $\square$ \\
\hline $\begin{array}{l}\text { action part } \\
\text { - code } \\
\text { - rules } \\
\text { - evaluadion } \\
\text { - intercuptible }\end{array}$ & $\begin{array}{c}+ \\
\text { vamiable } \\
-\end{array}$ & $\begin{array}{l}+ \\
- \\
-\end{array}$ & $\begin{array}{l}t \\
\square \\
\square \\
\square\end{array}$ \\
\hline $\begin{array}{l}\text { instantialions } \\
\text { - amount } \\
\text { - description }\end{array}$ & $\begin{array}{c}\infty \\
\text { ident., TSS name } \\
\text { trigg. cycle/event/ } \\
\text { decision } \\
\text { ratings, prionity }\end{array}$ & $\begin{array}{l}\text { immed. } \\
\text { exec. } \\
-\end{array}$ & $\square$ \\
\hline $\begin{array}{l}\text { Conbrolrycle } \\
\text { KS cycle } \\
\text { concumency } \\
\text { - parnildism } \\
\text { - distributed }\end{array}$ & $\begin{array}{l}+ \\
- \\
-\end{array}$ & $\begin{array}{l}-+ \\
+ \\
+ \\
+\infty\end{array}$ & + \\
\hline $\begin{array}{l}\text { matching } \\
\text { - event-based } \\
\text { - } 1 \text { K S-based } \\
\text { - amount. } \\
\text { number of activities }\end{array}$ & $\begin{array}{c}+ \\
- \\
\text { all. events } / \mathrm{KSs} \\
1\end{array}$ & $\begin{array}{l}-1 \\
\text { a.ll } \\
\text { all }\end{array}$ & $\begin{array}{l}+1 \\
\cdots \\
\cdots \\
\square\end{array}$ \\
\hline $\begin{array}{l}\text { Selection } \\
\text { - blackboand-based } \\
\text { - KS-based }\end{array}$ & + & - & $\mathrm{O}$ \\
\hline stop-criterion & $\begin{array}{l}\text { no inwoc. } \\
\text { KSARs }\end{array}$ & - & a \\
\hline
\end{tabular}

Table 7.4: Variation observed in BBL, AGORA, and GBB. 


\begin{tabular}{|c|c|c|c|c|}
\hline & Biondie & Blondie-II & Blondie-III & CASSANDRA \\
\hline $\begin{array}{l}\text { class-hieraurchy } \\
\text { panels } \\
\text { - hierarchy } \\
\text { levels } \\
\text { - hierarchy }\end{array}$ & $\begin{array}{l}- \\
>0 \\
>0 \\
-\end{array}$ & $\begin{array}{l}> \\
>0 \\
0 \\
>0 \\
-\end{array}$ & $\begin{array}{l}- \\
>0 \\
\square 0 \\
>\end{array}$ & $\begin{array}{l}- \\
>0 \\
>0\end{array}$ \\
\hline $\begin{array}{l}\text { units } \\
\text { - attr-value pairs } \\
\text { - typed } \\
\text { - hierarchy }\end{array}$ & $\begin{array}{c}\text { units } \\
+ \\
-\end{array}$ & $\begin{array}{c}\text { units } \\
+ \\
-\end{array}$ & $\begin{array}{c}\text { unitg } \\
\frac{1}{6} \\
-\end{array}$ & $\begin{array}{l}\text { entries } \\
+ \\
- \\
\end{array}$ \\
\hline $\begin{array}{l}\text { Tinks } \\
\text { - directed } \\
\text { - labelled }\end{array}$ & - & - & - & - \\
\hline $\begin{array}{l}\text { events } \\
\text { - structure } \\
\text { - abstraction } \\
\text { - units } \\
\text { - links } \\
\end{array}$ & $\begin{array}{c}\text { attr. value } \\
\text { pairs } \\
+ \\
+ \\
- \\
\end{array}$ & $\begin{array}{c}\text { altitr. value } \\
\text { pairs } \\
+ \\
+ \\
- \\
\end{array}$ & $\begin{array}{c}\text { attir. value } \\
\text { pairs } \\
+ \\
+ \\
-\end{array}$ & $\begin{array}{l}\square \\
\square \\
\square \\
- \\
\end{array}$ \\
\hline $\begin{array}{l}\text { interactions } \\
\text { - read } \\
\text { - create } \\
\text { - modify } \\
\text { - delete } \\
\end{array}$ & $\begin{array}{l}t \\
+ \\
+ \\
+\end{array}$ & $\begin{array}{l}+ \\
+ \\
+ \\
+\end{array}$ & $\begin{array}{l}+ \\
+ \\
+ \\
+ \\
\end{array}$ & $\begin{array}{l}+ \\
+ \\
+ \\
+ \\
+\end{array}$ \\
\hline $\begin{array}{l}\text { description } \\
\text { - binding } \\
\text { - features }\end{array}$ & + & + & + & $\begin{array}{l}\square \\
\square \\
\square\end{array}$ \\
\hline $\begin{array}{l}\text { condition } \\
\text { - trigger } \\
\text { - precondition }\end{array}$ & + & + & + & $\begin{array}{l}\square \\
\square \\
\end{array}$ \\
\hline $\begin{array}{l}\text { action part } \\
\text { - code } \\
\text { - rules } \\
\text { - evaluation } \\
\text { - interruptible }\end{array}$ & $\begin{array}{c}+ \\
- \\
-\end{array}$ & $\begin{array}{l}+ \\
- \\
- \\
\end{array}$ & $\begin{array}{l}+ \\
+ \\
0 \\
-\end{array}$ & $\begin{array}{r}- \\
+ \\
+ \\
-\end{array}$ \\
\hline $\begin{array}{l}\text { instantiations } \\
\text { - amount } \\
\text { - description }\end{array}$ & $\begin{array}{l}\infty \\
\text { trig. unit } \\
\text { trig. event } \\
\text { precond. } \\
\text { binding } \\
\text { features } \\
\text { priority } \\
\end{array}$ & $\begin{array}{l}\infty \\
\text { trig. unit } \\
\text { trig. event } \\
\text { precond. } \\
\text { binding } \\
\text { features } \\
\text { priority } \\
\end{array}$ & $\begin{array}{l}\text { Do } \\
\text { trig. unit } \\
\text { trig. event } \\
\text { precond. } \\
\text { binding } \\
\text { features } \\
\text { priority } \\
\end{array}$ & $\begin{array}{l}\square \\
\text { entry } \\
\text { KS iddent. }\end{array}$ \\
\hline $\begin{array}{l}\text { control cycle } \\
\text { KS cycle } \\
\text { concurrency } \\
\text { - parallelism } \\
\text { - distributed } \\
\end{array}$ & $\begin{array}{l}+ \\
- \\
- \\
-\end{array}$ & $\begin{array}{l}+ \\
+ \\
+ \\
+\end{array}$ & $\begin{array}{l}+ \\
+ \\
+ \\
+ \\
+\end{array}$ & $\begin{array}{l}+5 \\
+ \\
+ \\
+\end{array}$ \\
\hline $\begin{array}{l}\text { matching } \\
\text { - event-based } \\
\text { - KS-based } \\
\text { - amount } \\
\text { number of activities }\end{array}$ & $\begin{array}{c}+ \\
\cdots \\
1 \\
\end{array}$ & $\begin{array}{c}+ \\
- \\
\text { all } \\
1 / \text { processor } \\
\end{array}$ & $\begin{array}{c}t \\
\cdots \\
\text { all } \\
1 / \text { node }\end{array}$ & $\begin{array}{l}0 \\
0 \\
0 \\
0\end{array}$ \\
\hline $\begin{array}{l}\text { Selection } \\
\text { - blackboard-based } \\
\text { - IKS-based }\end{array}$ & $t$ & + & $\begin{array}{l}+ \\
+ \\
\end{array}$ & ㅁ \\
\hline stop-criterion & bb pattern & Bb pattern & bD pettern & $\square$ \\
\hline
\end{tabular}

Table 7.5: Variation observed in Blondie, Blondie-II, Blondie-III and CASSANDRA. 
blackboard

\begin{tabular}{|c|c|}
\hline orgenisisation & \\
\hline clasis herarchy & $-x+$ \\
\hline perancis & $n_{1}>0$ \\
\hline - hierardhy & - Partial abstraction \\
\hline levels & $\mathrm{n},>0$ \\
\hline = hierarchy & , class, ordered, partially ordered \\
\hline uanitis & \\
\hline - form & attribute-value pains, typed \\
\hline - hierarchy & - ordered \\
\hline links & $-1,+4$ \\
\hline - directed & -+ \\
\hline - labelled. & - t, reduction/expectation \\
\hline ewents & \\
\hline structure & - (token), attribute-value pairs \\
\hline semantics & state change, clock, problem (dynamic trigger deff), goal \\
\hline abstraction & $-1+$ \\
\hline units & \\
\hline links & $-1+$ \\
\hline Interactions & \\
\hline read & + \\
\hline create: & + \\
\hline modify & $-{ }_{4}+$ \\
\hline delete & -4 \\
\hline consistency & $-m_{4}+1$ \\
\hline & knowledge sources \\
\hline description & \\
\hline $\begin{array}{l}\text { binding } \\
\text { attributes }\end{array}$ & $\begin{array}{l}\text { +. LHSs, immediate code, precondition, stimulus frame } \\
\text { levels, links, rule evaluator, variables, features }\end{array}$ \\
\hline coridition & \\
\hline $\begin{array}{l}\text { trigger } \\
\text { precondition }\end{array}$ & $\begin{array}{l}+ \text {, list of events, denons, event evaluation, goal, token } \\
-, 4 \text {, comb. of predicates }\end{array}$ \\
\hline action part & \\
\hline $\begin{array}{l}\text { code } \\
\text { rules } \\
\text { - rule evaluation } \\
\text { intertuptible }\end{array}$ & $\begin{array}{l}-, \$ \\
-,+ \text { parallelism } \\
\text { parallel, variable } \\
-\end{array}$ \\
\hline instantiations & \\
\hline $\begin{array}{l}\text { amount } \\
\text { description }\end{array}$ & $\begin{array}{l}\text {-, l, unestricted } \\
\text { - entry \& KS, response \& stimulus frame, } \\
\text { scheduhing unit, KSAR }\end{array}$ \\
\hline & control \\
\hline basic cycle & \\
\hline $\begin{array}{l}\text { Control cycle } \\
\text { KS cycle }\end{array}$ &,,$-+ \square$ \\
\hline concuntericy & \\
\hline $\begin{array}{l}\text { concurrency } \\
\text { - parallel } \\
\text { - Clistributed }\end{array}$ & $\begin{array}{l}-1+ \\
-x+ \\
-\cdots+\end{array}$ \\
\hline matoluing & \\
\hline event-bersed & + \\
\hline KCS-based & 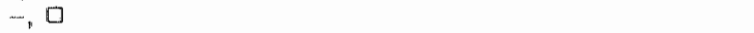 \\
\hline amount & 1, 1 to all, all, variable, all clock/all problem/1 event \\
\hline number of activities & 1,1/processor, $1 /$ node, variable \\
\hline selection & \\
\hline $\begin{array}{l}\text { selection } \\
\text { - blackboand-based } \\
-1 \% 5 \text { based }\end{array}$ & 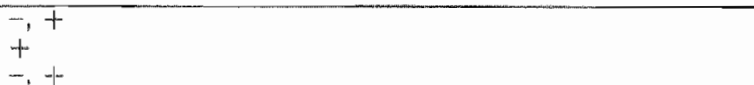 \\
\hline stopm-criterion & $\begin{array}{l}\text { blackboard condition, empty event list, } \\
\text { no invocable } K S \text { s, stop KS }\end{array}$ \\
\hline
\end{tabular}

Table 7.6: Summary of options observed in the fourteen blackboard architectures. 
Units. All blackboard architectures recognise basic elements containing the information on the blackboard. Many names are used for these elements (units, elements, hypotheses, hypothesis elements, nodes, entries), but they are all either data types or sets of attribute-value pairs. We view the difference between units defined as data types or as sets of attribute-value pairs as follows. Sets of attribute-value pairs are defined more loosely than data types and can be redefined easily during runtime. The stricter use of data types allows implementation of facilities such as dynamic type checking.

In one case $(\mathrm{GBB})$, units are ordered according to dimensions according to their attributes. Ordering of units can influence efficiency of data. access on the blackboard greatly.

Links. Even when links can not be expressed explicitly, the possibility of specifying links as attributes of units always exists. Certain blackboard architectures, however, provide explicit facilities for representing links. Both labelled and unlabelled links as well as directed and undirected links are supported by different architectures. Explicit representation of links facilitates their use in consistency maintenance (of the links themselves, but also of linked units) and in event specifications.

\section{Interactions}

Clearly, it must be possible to write information on the blackboard and to read information from the blackboard. Yet, there exists some variation in the allowed interactions with the blackboard.

Read. Reading of information is indispensable. Section 7.2 discusses further the relevant variation.

Create. Analogous to reading, the possibility of creating units on the blackboard is indispensable.

Modify. In one case (AGORA) the modification of already created elements on the blackboard is not allowed. Modification is important to represent additional information about already known facts or objects or to model a changing world state.

Delete. Deletion of existing elements is only allowed in a small number of blackboard architectures. Deletion is mainly useful as a form 
of garbage collection (deleting obsolete information) and to allow nonmonotonic reasoning about items represented on the blackboard (deleting hypotheses which have become false).

Consistency. Examples of facilities for maintenance of consistency of data on the blackboard can be found in AGE (in the form of a calculus for certainty factors, automatically updating certainty factors in light of newly created information) and $\mathrm{GBB}$ (for maintaining bidirectionality of links).

\section{Events}

Events are important in blackboard architectures for matching the conditions of KSs with information on the blackboard more efficiently. Also, events form a representation of a condition in terms of blackboard changes which can be a 'natural' way of expressing interesting new situations in the condition of KSs in certain applications.

Implementation. In the blackboard architectures we examined, events are either implemented as tokens or as attribute-value pairs. Generally, tokens (also called labels: names to indicate a specific interaction with the blackboard) can be more efficiently matched with conditions, but are much less flexible than more descriptive events and also necessitate a clear a priori understanding of which events are relevant.

Expressibility. An event normally represents a change on the blackboard and can be matched with the condition of KSs. In DVMT events are used also to derive goals for the problem-solving process. The system HASP/SIAP uses two special ways of determining applicability of KSs: via clock events and problems. Clock events specify that it is time to execute a certain task. Problems can be seen as dynamically generated (by a KS) triggers, which specify that when a certain situation occurs, the associated task (for instance the remainder of the KS that posted the problen) must be executed. POLIGON allows activities to be triggered even when only a semaphore is being waved at a certain unit or unit attribute, but no actual modification takes place.

Abstraction. In most blackboard architectures (but not all) an event forms only an abstracted description of the corresponding blackboard interaction. 
Units. In all cases events can represent modifications of blackboard units.

Links. Only in some architectures, can events be used to represent modifications of links too.

\subsubsection{Knowledge sources}

In this section on variation of knowledge sources, we address, in turn, KS descriptors, condition parts, action parts, and instantiations.

\section{Descriptor}

The most primitive information kept about a KS in a KS descriptor is a name (in POLIGON). However, in most blackboard architectures more information about a KS is represented. Apart from references to the corresponding condition and action parts, this information can be subdivided into information about how binding occurs of a KS with the context in which it became applicable and information (features) that can be used for control.

Binding. Binding occurs for instance via the execution of immediate code upon instantiation (Hearsay-III) or by the precondition of a KS (in BB1 and the three Blondie shells). The results of binding are stored in special data structures representing an instantiation (stimulus frames in Hearsay.II, units on the control blackboard in Hearsay-III, or KS activation records in $B B 1$ and the Blondie shells). AGE and CAGE provide a facility for binding of variables by the left-hand sides (LASs) of the rules in the corresponding action part for use by other rules in the same action part.

Features. Other features of KSs included in KS descriptors are: refarences to the blackboard levels and panels accessed by the KS action parts; the links to be addressed by the action parts; the variables involved; the rule evaluator to be used when the action part consists of a collection of rules; and features, such as efficiency and credibility. In general, features are used to provide extra information regarding applicability of a ISS and the quality of expected results. 


\section{Condition part}

A condition is indispensable for each KS in every blackboard system. One condition can be used for more than one KS. Often (Tables 7.2 , $7.3,7.4$, and 7.5 show in which cases), a subdivision of the condition is made into a trigger (specifying interesting events) and a precondition (specifying interesting blackboard states).

Trigger. Some variation exists in the way triggers are defined in those blackboard architectures using triggers. In systems where events are represented via tokens, the trigger consists of a token or a list of tokens (AGE and CAGE). In more general cases, a trigger is represented as a list of events (possibly with 'don't care' options, see also the discussion about possible variation in Section 7.2). BB1 uses an event evaluator which can submit a produced event to an arbitrary complex test. Blondie-III allows triggers to be defined in terms of combinations of events within the same cycle or subsequent cycles, formed by the constructors AND, OR, and FOLLOWED-BY. In POLIGON triggers are implemented in a demon-like fashion: updating of certain values on the blackboard results in immediate execution of corresponding activities. Finally, DVMT uses, among others, an event-goal table to derive goals for the problem-solving process from events and a goal-KS table to determine applicable KSs.

Precondition. Preconditions are usually defined less strictly than triggers. The task of a precondition is to determine applicability based on all relevant and available information, notably the information on the blackboard. Preconditions in the examined blackboard architectures (when present) are either implemented as a procedure to establish them or represented as (arbitrarily complex) combinations of predicates.

\section{Action part}

The examined blackboard architectures mostly allow either rules or procedures (functions) as the action parts of KSs. The exceptions here are HASP/SIAP and Blondie-III. HASP/SIAP uses procedures, rules, and frames for representation of knowledge in KSs. In Blondie-III both rules and procedures can be used for the action parts. Thus the inherent possibilities of the blackboard model for incorporating diverse knowledge representation and reasoning methods has not been explored much in 
these blackboard architectures. As the contents of KSs in a blackboard system are typically application oriented, these contents are hardly of interest in this discussion.

Code. Blackboard architectures with procedural action parts can also use rule-based processing within their action parts by implementing a rule evaluator as an internal procedure.

Rules. The observed variation in the KS action parts of blackboard architectures allowing rule-based processing can be found in two dimensions: rule evaluation and concurrency. In $\mathrm{AGE}, \mathrm{CAGE}$, and $\mathrm{BB} 1$, the way in which rules are fired, in addition to when and how often, can be specified by the user. CAGE adds to this flexibility by allowing the user to specify when rules, clauses in rules, and statements within clauses can be evaluated in parallel.

Interruptible. None of the examined blackboard architectures allows any interruption of the execution of $\mathbb{K S S}$.

\section{Instantiations}

Usually, KSs can be seen as functions. A KS takes certain information as its input and performs actions dependert on this input. During the problem-solving process, one KS may be executed more than once; each time with different information as input. The pair consisting of a KS and an appropriate input (context or representation of the blackboard state that made the KS applicable) is an instantiation for that KS. Not all blackboard architectures use KS instantiations explicitly (see also Section 7.1.4).

Amount. A basic difference in the amount of $\mathrm{KS}$ instantiation that can exist at the same time in a blackboard system is related to the mechanism used for selecting KSs for execution. Systems such as HASP/SIAP, $A G E$, and $C A G E$ first select an event or a number of events and then proceed by executing all KSs that have a condition matching the selected event(s). Thus, during processing of a selected event, at most one instantiation of a $\mathrm{KS}$ is considered. The other blackboard architectures have no apparent restriction on the amount of instantiation of a $K S$ that can exist simultaneously. 
Description. KS instantiations are represented within blackboard systems in various ways. Hearsay-II constructs a stimulus frame as well as a response frame for instantiations (see Appendix A for details), representing information about the instantiating blackboard states or changes and expected results, respectively. Hearsay-III stores descriptions of instantiations as units on a control panel of the blackboard. CASSANDRA stores for each instantiation just the triggering blackboard unit and the name of the KS. BB1 and the Blondie shells create KS activation records (KSARs) containing all information specific to a $\mathrm{KS}$ instantiation and relevant for control.

The information represented for an instantiation can be used for reasoning about the actions of the blackboard system. The amount of information partly determines the sophistication of control in a blackboard system (although it need not be clear how this information can be applied most effectively).

\subsubsection{Control}

Our discussion of observed variation of control addresses the following aspects: basic cycle (control cycle or KS cycle), concurrency, matching, selection, and stop criterion.

\section{Basic cycle}

The blackboard metaphor describes specialists gathered around a blackboard. The specialists perform a cycle of alternately monitoring the blackboard for interesting information and contributing to the problemsolving process when an interesting situation has occurred. We call this cycle a $K S$ cycle to distinguish it from the control cycle executed by a control unit (see also Chapter 6).

Control cycle. Most blackboard architectures not employing massive parallelism need explicitly to schedule applicable KSs in order to mediate between KSs competing for execution on the restricted number of processors. Thus, all examined blackboard architectures but AGORA and POLIGON use a control cycle.

KS cycle. AGORA and POLIGON (and also CAGE in its configuration exploiting parallelism most extensively) are implemented using a KS 
cycle: they resemble the blackboard metaphor more closely than the other examined architectures.

\section{Concurrency}

A distinction is made between parallel blackboard architectures and distributed blackboard architectures. Parallel blackboard architectures are characterised by a shared-memory blackboard (and concurrently executed KSs), while in distributed blackboard architectures the blackboard and control are distributed. Often a distributed blackboard system is equal to a collection of (sequential) blackboard systems together with mechanisms for communication between the individual blackboard systems. Evidently, blackboard systems can be distributed in a fashion which is a combination of these two different approaches.

Parallel. CAGE and Blondie-II are both parallel blackboard architectures. In CAGE execution of KSs can be synchronised.

Distributed. DVMT, Blondie-III, CASSANDRA, and AGORA are distributed blackboard architectures. DVMT and Blondie-III both consist of cooperating complete blackboard systems. CASSANDRA supports cooperation between Level Managers, each responsible for one blackboard level and KSs operating on that level. AGORA is a framework for distributed problem solving supporting the development of, among others, distributed blackboard architectures.

\section{Matching}

Each blackboard architecture needs to inchde a mechanism for matching conditions of KSs with whatever information is deemed relevant. for determining the applicability of KSs. How this is done and what lind of information is used may vary. A distinction is made between blackboard architectures which take events and search for KSs matching these events (event-based matching), and blackboard architectures which consider KSs and search (periodically) for events that match the KSs (KS-based matching).

Event-based. Almost all examined architectures use an event-based matching orientation. $\mathrm{CAGE}$ can be an exception: in certain configurations CAGE exhibits $\mathrm{KS}$-based matching. 
KS-based. KS-based matching seems to be most appropriate in architectures using a KS cycle. In fact, CAGE can exhibit KS-based matching when a configuration is used in which all applicable KSs are executed immediately without any synchronisation. This is also the configuration where flow of control in CAGE follows a KS cycle instead of a control cycle. However, AGORA and POLIGON, both with a KS cycle, basically employ event-based matching.

Amount. Depending on whether event-based matching or KS-based matching is used, the number of events, respectively KSs, matched each time, can be examined. In AGE only a single event is matched. In HASP/SIAP all previously selected events are matched as well as all expedient clock events and problems. In CAGE the number of the total number of events that are matched may vary between only one and all events. In the other examined architectures all events which occurred intermediately are matched with all present KSs. Variation of the number of matched events or KSs may be useful when a blackboard architecture is used for real-time applications (see also Section 7.2).

Number of activities. Another aspect of variation among the examined architectures is the number of activities selected each time. Usually, when a blackboard system uses selection, only one activity is selected (in Hearsay-II activities can be execution of a KS action part as well as evaluation of a $K S$ condition part). In AGORA and POLIGON all applicable activities are 'selected' and executed. HASP/SIAP and CAGE both allow the user to specify the number of selected activities. Blondie II selects one activity for each idle processor, while DVMT and Blondie-III select one activity at a time for each node.

\section{Selection}

The examined architectures show great diversity in the kind and amount of information used for selecting activities for execution. Such diversity stems from different application details. We concentrated in our analysis on the question of whether selection is based on information held by the b]ackboard and/or on information about the KSs. When enough processing capacity is available, selection of activities for mediating between activities competing for limited resources is not necessary (although selection might be needed for deciding which activities are effective). In 
the design of AGORA and POLIGON, it is presumed for this reason that no mediation between competing activities is needed. It appears that only HASP/SIAP, AGE, and CAGE have completely blackboard-based selection of activities. Usually, KS-based selection is also employed.

\section{Stop criterion}

Each current blackboard architecture halts automatically when there are no more applicable KSs and no KS is being executed currently. Additional mechanisms have been implemented to determine whether the system is allowed to stop in a more sophisticated manner. Several blackboard architectures include a procedure in the control cycle for deciding whether a certain stop criterion has been met. In AGE and CAGE, as well as in the Blondie shells, this stop criterion is formulated in terms of the contents of the blackboard. Hearsay-II uses a dedicated KS which removes unlikely hypotheses from the blackboard, ultimately ensuring that no KS instantiations for processing other hypotheses remain after a useful solution has been found.

\subsubsection{Observed correlations between different aspects}

Whenever two different options are observed only simultaneously, it suggests that these options are in some way related. Evidently, the sample of fourteen blackboard architectures is too small to deduce such pertinent relationships, but one may expect that in most cases additional arguments could be found confirming such indications.

In general, there seems to exist a correlation between the following six options:

- selection

- features in KS descriptors

- contral cycle

- instantiations

- binding for instantiations

- stop criterion

These options occur in cases where time and/or processing capacity constraints are evident (i.e., in all examined systems but POLIGON and 
AGORA). The argument runs approximately as follows: constraints necessitate selection; selection needs to weigh factors - e.g., KS features; selection is best done by an independerit component, i.e., a control unit, executing a control cycle; a control cycle will delay certain $\mathrm{KS}$ activities until much later, therefore, currently applicable activities should be represented (via instantiations, descriptors); in order to have access to the situation enabling an activity, binding seems appropriate; when there is a control cycle, it is convenient to include a stop criterion as well.

\subsection{Extending the existing options and varia- tions}

In this section we review the variation in existing blackboard architectures as described in Section 7.1 and try to determine when, where, and in what way that variation can be extended. This discussion is intended to give answers to two of the questions posed in the Chapter 1 .

Firstly, we are interested in new and useful options and variations. In orcler to assess the possible usefulness of options and variations, we need to cliscuss the semantics of each such option (old and new). This section presents such discussions. We have not indicated in these discussions whether a certain variation is new as a matter of fact, since there might already exist a blackboard architecture which includes just that variation, but did not come to our attention. The discussion of the semantics of variations new to us is necessarily speculative, as we have not experimented with them. A review of some, possibly "new', options is given separately in Section 7.2.5.

Secondly, this section addresses, indirectly, the issue of which options and variations to choose for a specific application. The discussion of semantics of possible variations can be used by a blackboard-system developer to determine which options should be selected to obtain a certain desired characteristic.

The discussion in this section follows roughly the aspects as listed in Table 7.6 . 


\subsubsection{Blackboard}

The discussion of possible variation of the blackboard is subdivided into discussions of the purpose and/organisation of the blackboard, interaction with the blackboard, and events.

\section{Purpose of the blackboard}

We first examine the purpose of the blackboard. Primarily, the blackboard is a medium for asynchronous communication between otherwise independent knowledge sources. But the blackboard has been used also as a data structure for representing the current problem-solving state. In some applications (e.g., HASP/SIAP for sonar interpretation and Blondie-II for robot control) the blackboard was used for representing (among other things) a current world model. The use of the word "current" in these sentences immediately suggests other uses of the blackboard. One of such uses is representation of future problem-solving states or future world models (which may be compared to the representation of goals, as in DVMT). Two other applications of the blackboard might be the representation of future activities (or plans; also in DVMT) and the representation of a history of activities or problem-solving states (sometimes present in existing blackboard architectures in the form of links, which may be used to represent inference relations). Finally, the blackboard can be used as a 'scribbling pad' for KSs to (temporarily) store internal findings (e.g., for use by later instantiations of the same KS) or even to store expert knowledge, allowing it to be readable and possibly modifiable by other KSs. Some observations can be made with respect to the blackboard in each of these different uses (which, by the way, may be combined in various ways).

Communications medium. When the blackboard is used as a communications medium, regions (levels, classes) of the blackboard can be seen as communication channels, while the entities on the blackboasd are data elements sent from one KS to other KSs.

The blackboard as a medium for communication is used mostly in a broadcasting manner: the complete blackboard is writable by all KSs and also all information on the blackboard is readable by all KSs. By restricting the read and write access rights for a region on the blackboard 
to particular KSs, a multicasting or even singlecasting communication scheme could be implemented. In (Yang et al, 1985); a distributed blackboard architecture is described which allows a blackboard to be used for both broadcasting and singlecasting communication.

A symmetric definition of read and write access allowing a set of KSs to both read and write the same set of regions leads to bidirectional communication channels between involved KSs. Likewise, the asymmetric definition of read and write access leads to unidirectional communication channels.

Another interesting variation might be single use (removal upon reading) or multiple use of information. Single use of information might be useful in cases where a blackboard entity signifies a task to be performed. Another application of single use would occur when certain entities may be used only once as part of other hypotheses or (partial) solutions.

Description: current problem-solving state (CPS). Viewed as representing the current problem-solving state, the entities on the blackboard correspond to problem-solving elements such as hypotheses, but also to control information, such as policies and strategies.

Often useful in this context, is the use of links to administer inference steps and register dependencies.

In this case, a hierarchy of regions (levels, classes) emerges corresponding to an appropriate problem-solving hierarchy (cf. for instance the level hierarchy in the speech recognition system Hearsay-II).

Usually, the existence of conflicting hypothesis elements is allowed explicitly, but a mechanism for specifying and manipulating present beliefs seems to be needed as well.

Use of the blackboard in this manner has proved to be successful in domains such as pattern recognition, planning, and design.

Description: current world model (CWM). When a blackboard is used for representing a current world model, many insights can be derived from other existing modelling techniques, most notably objectoriented modelling. Not surprisingly, several blackboard frameworks (e.g., Hearsay-III and POLIGON) provide mechanisms for defining data on the blackboard also found in object-oriented modelling, such as objects, classes, and inheritance. In this case, classes form the partitions 
on the blackboard and class hierarchies emerge, such as abstraction and component or aggregation hierarchies.

The idea of using the blackboard for representing a current world model should restrict permissiveness towards holding conflicting hypotheses simultaneously: in a current world model something must be believed to be true, false, or unknown.

Blackboard architectures with a blackboard holding a current world model have been applied mostly in application areas such as monitoring and process control.

Description: future problem-solving state (FPS). A blackboard containing future problem-solving states (desired or predicted) is similar to a blackboard containing goals. Some examples exist of blackboard architectures representing and reasoning about goals. See Chapter 8 for a discussion of such examples.

Description: future activities (FA). Dual to a representation of future states is the representation of future activities expected to transform future states. This coincides with a representation of plans or agendas.

Distributed Artificial Intelligence research has recognised the advantages of explicitly representing plans for optimising cooperation between agents. See, e.g., (Durfee, 1988) for the use of 'partial global plans' in a. distributed blackboard architecture.

Description: history. A representation of previously derived results and performed activities can be beneficial to deciding what activities to take next. In a blackboard system such as Hearsay-II, where information can be added or modified, but not deleted, a history of earlier derived hypotheses remains, which can be useful, e.g., for backtracking.

KS scribbling pad. In the blackboard metaphor, IKSs are seen as specialists performing their specialist tasks internally and communicating to the blackboard only partial results that could be of interest to other specialists. Implementations, however, often use KS instantiations performing their defined activities after which they cease to exist. A specialist, however, will have the results of earlier internal calculations still available, even when the specialist is working on different subproblems later during the problem-solving process. Results of internal calculations by a KS instantiation possibly beneficial to other instantiations of the same KS, however, are lost when the KSI ceases to exist, unless 
this information is recorded, e.g., on the blackboard. This leads to the possible use of parts of the blackboard as a kind of 'scribbling pad' for a. KS.

KS expertise. Normally, a $\mathrm{KS}$ is seen as an independent module, and its workings (or "expertise") is kept hidden from the outside. But it might be a useful extension to make (parts of) this expertise available to other $\mathrm{KSs}$ for inspection or even modification. In this way, KSs could make informed decisions about the contributions to be expected from other KSs or improve the functionality of other KSs by reformulating their expertise. Expertise (e.g., a collection of rules) could be made accessible for this purpose by placing it on the blackboard.

It is remarkable that, although the primary function of the blackboard would be to provide a means for communication, consequences and opportunities related to this view have not been given much attention yet. Much more stress has been given to the role of the blackboard in representing either problem-solving state or current world model (albeit in the last case often in a diluted version: information relevant to the problem-solving state or history are included easily). ${ }^{1}$ This concludes our discussion of possible purposes of the blackboard. In the following section we continue with a discussion of possible ways of organising data on the blackboard.

\section{Organisation}

Since, in the course of a problem-solving process' execution, it is usually necessary for a grest deal of data to be stored on the blackboard, organisation of data on the blackboard is necessary for increasing efficiency in accessing specific data elements.

Entities. Usually, the basic entities on the blackboard (units, hypothesis elements, etc.) are implemented either as attribute-value pairs or as types. Attribute-vallue pairs are more flexible in use, as the set of attributes can be augmented and sometimes modified without too many consequences. The advantage (but in some cases disadvantage) of a

\footnotetext{
${ }^{1}$ In this context, it is interesting to note again that the difference between using the blackboard for representing either the problem-solving state or the current world model is related to the distinction between an Assumption-based Truth Maintenance System and an ordinary Truth Maintenance System (see page 11).
} 
typed representation of entities on the blackboard lies in runtime type checking, as it allows to keep data and its use consistent with its type definition automatically.

Organisation Arguments for organising entities on the blackboard in a certain way are related to the role assigned to the blackboard as described in the previous section (as communication medium; for representing current problem-solving state, etc.). In many blackboard architectures entities are grouped together in levels, which, in turn, are combined to form panels. This partitioning can be extended by introducing object-oriented classification of entities, which has been used in some blackboard architectures. Object-oriented modelling seems sufficiently rich to provide a general framework for organising the blackboard.

Relations. Additional organisation on the blackboard can be obtained by representing relations between different entities explicitly. Binary relations (often called 'links') are commonly used in blackboard architectures. Links are used to represent causality, or dependencies, or to indicate part-whole relationships. In the case of binary relations, a distinction between directed and undirected links makes sense, where direction is used mostly to indicate support (following the direction of the inferencing process). Apart from binary relationships, also $n$-ary $(n>2)$ relationships might be interesting for organising data on the blackboard, e.g., to represent aggregation hierarchies. Labelling of relations enables the use of different relations for different purposes within one system.

Dynamic definition. Although the number of entities on the blackboard, and, optionally, the place where these entities are stored, is flexible, normally the organisation of the blackboard is defined statically. This assumes that it is clear at the outset which classes of entities are to be communicated. But this need not be the case necessarily. It is imaginable that in some applications the structure of the blackboard can be defined only during runtime as a world of discourse is formed among the $\mathrm{KSs}^{2}$. An example of this idea can be found in the Ariadne-I system (Craig, 1986).

\footnotetext{
${ }^{2}$ This idea is analogous to an aspect of situated action (Agre, 1990 ), where decisions with respect to the behaviour of a system are derived from ongoing interaction between the system and its enviromment, thus continaously upditing the system's view of its goals and tasks.
} 
Different blackboards. Conceptually and organisationally the blackboard may be divided into a number of blackboards. This notion coincides with the use of blackboard panels, for instance for representing different hierarchies. An even stricter division becomes interesting for concurrent blackboard systems when the blackboard is distributed over different processes or processors. This is especially useful, when blackboard space and/or access prove to be efficiency bottlenecks.

\section{Interactions}

In this section we indicate possible ways of interacting with a blackboard. Creation of entities on the blackboard and reading the contents of the blackboard are essential interactions. Most blackboard architectures add the possibility of modifying entities and some even allow the removal of entities or transportation of entities from one class (level) to another.

Creation. As indicated, creation of entities is an essential interaction. functionality for a blackboard. Variation for this interaction is related directly to the variation allowed for entities on the blackboard.

Reading. There are many ways of reading data from the blackboard. The most obvious is via call-by-name. But often, data needs to be retrieved fulfilling some characteristic and call-by-name is not appropriate. So, most blackboard frameworks provide means for 'associative' data retrieval from the blackboard. Actually, the possible variation is determined by the possible variation in matching per se. We discuss matching variation in more detail in the following section.

Matching variations. The most trivial form of matching is exact matching: two items match if and only if they are equal. Abstraction leads to a more permissive form of matching: two items match when their abstracted forms are equal. Abstraction can, e.g., be implemented via wildcards or 'don't care' options. This kind of abstraction either disregards the values for certain attributes for an item or matches items when values for a certain attribute are in the same set (a subset of the domain for that attribute).

These forms of template matching all presuppose set theory based on a two-valued logic. Fuzzy sets (Sombé, 1990) allow for even more variation when applied on the matching criteria as mentioned previously. 
Modification. Modification of entities can be achieved either by a dedicated modifying interaction or by the successive application of removal and creation interactions. The latter option can have some disadvantages as it normally leads to a new entity (with a different identity) and might interfere also with triggers monitoring removal and/or creation of entities in the involved regions of the blackboard.

Removal. Not all blackboard architectures allow the removal of entities from the blackboard. But removal can be useful as a form of garbage collection and for nonmonotonic reasoning activities (removing entities no longer thought to be valid). In the CASSANDRA architecture, removing entities from the blackboard was seen as a knowledge sensitive task and was restricted therefore to dedicated KSs.

Transportation. Consider a blackboard application in which some entities on the blackboard model real-world objects and different regions (levels, classes) on the blackboard represent different areas or classes in the real world. In such an application, moving an object from one area to another or assigning an object to another class should be modelled by the transportation of the corresponding entity on the blackboard from. one blackboard region to the appropriate (different) region. This leads. to the definition of blackboard interaction which transports blackboard entities from one region to another. In such an interaction, the entity keeps its identity, but can have some of its attributes changed or be given completely new attributes. The blackboard framework GBB is an example of a system allowing transportation of entities.

Multiple use. As modifications of the blackboard can result in creation of events and consequently in the generation of KSIs (tasks), it is conceivable that a certain modification results in triggering several tasks all applicable in this situation, but not all those necessary (for instance when one task is subsumed by another). These and analogous considerations might suggest implementation of a mechanism restricting the number of uses of an entity. When an entity is marked for singie use only, it should not be read, used for triggering, or included in a solution (depending on the definition of use) more than once. Other forms of restricted use, ranging from single use to unrestricted use, can be defined easily. The last variation can be observed in a mechanism called consumption in Hearsay-III.

Restricted access. As seen while viewing the blackboard primarily as 
a communications medium, restricting access of certain regions of the blackboard to a selected number of KSs can be meaningful. Restricted access can be useful for increasing efficiency of interactions as well, as restricted access automatically preselects regions for access.

A dynamic form of restricted blackboard access is useful in concurrent implementations for (temporarily) locking certain regions of the blackboard and keeping the contents of the blackboard consistent. This locking mechanism has been provided, e.g., in the parallel blackboard shell Blondie-II.

Automatic consistency maintenance. Checking of consistency and maintaining consistency can be useful in general. Entities on the blackboard are created based on qualitative and quantitative information held in other entities. As entities are modified, the credibility of derived entities might be violated. An obvious example (already present in HearsayII) is updating currently held beliefs in interrelated hypotheses as the belief in one hypothesis on the blackboard is changed. Another example is maintaining a consistent set of links among entities which together form some relationship. Providing tools for automatically updating related values on the blackboard was considered in AGE (in the form of an implementation of preprogrammed procedures for MYCIN's calculus of Certainty Factors).

Dynamic interaction definition. Usually, interaction by the $\mathbb{K} S \mathrm{~S}$ with the blackboard is predefined. Consider, however, a situation where interaction is not predefined, but is determined during runtime. Especially in a blackboard system where the liSs are themselves agents within the system, making informed decisions with respect to the information to be placed on, or to be read from, the blackboard is an interesting concept. A KS could for instance observe that certain kinds of information are never used by other KSs, and conseguently decide not to spend any more effort in obtaining those kinds of information. Speculations in this direction are more a topic of Distributed Artificial Intelligence research and is not explored here any further.

Dynamic blackboard-organisation definition. When the organisation of the blacliboard is made dynamic, interaction facilities have to be supplied for modifyng a current blackboard organisation via, e.g., creation and deletion of regions (levels, classes), modification of the set of attributes for entities in a specific region, etc. 


\section{Events}

The final group of possible variations concerned with the blackboard in blackboard systems deals with events. The possible variation of events is related directly to the variation in kinds of information represented on the blackboard (entities, attributes, links, relations, etc.) and the permitted interactions. This includes the possible creation of events for all forms of modification, but also for read access only.

Additional variation can be achieved by allowing various levels of $a b-$ straction to be used in the specification of events.

In some cases, it might be interesting to note if certain events have not taken place, e.g., in situations where timers need to be set checking for the timely occurrence of certain activities.

The use of events is discussed later on in more detail in the section on the flow of contral.

\subsubsection{Knowledge sources}

Our discussion of possible variation for $\mathbb{K} S$ s addresses, in turn, organisation of KSs, condition parts, action parts, and a collection of additional. variation opportunities.

\section{Organisation}

Knowledge sources tend to combine a collection of (elementary) activities. These activities are grouped together according to different views. The activities in a KS usually share a common element making that KS (or the activities collected in the KS action part) applicable. This common element is in fact what initiates the task represented by a KS instantiation, as it was defined in Chapter 6. We call this common element the initiator. The initiator for a KS can be any item from the following list:

situation A situation is actually a description of the blackboard state in which the activities of a KS can be applied effectively. 
event An event is a blackboard state change and can make a KS become applicable as well.

object An object (e.g, an entity on the blackboard) can be used as initiator when all activities are combined in a KS that can be applied on a specific object. This approach resembles the methods of an object in object-oriented programming, albeit at a different level of granularity.

goal A goal represents a result of activities. Instead of concentrating on opportunities for contribution, a viable option for combining activities into a $\mathrm{KS}$ might be to take those activities that together achieve a certain (sub)goal.

The initiator for a KS plays a central role in the condition of a KS, although it need not be sufficient for defining a condition. We note that conditions are often stated in terms of combinations of different kinds of initiators (situations and events; goals and situations), while on the other hand initiators are usually represented only by abstractions of these initiators. If we re-examine the blackboard metaphor, it strikes us that originally KSs tended to be defined more in terms of combining a certain body of expertise, than being collected around a specific initiator. However, for each $\mathbb{K S}$ a condition specifying when its activities can be applied is indispensable. Hence, identification of an element which could serve as initiator becomes also necessary.

\section{Condition part}

Evidently, each KS must have assigned to it, apart from the activities constituting its action part, a means for assessing its applicability during the problem-solving process. So, broadly speaking, a KS is necessarily a. condition-action pair. But there exist many variations both in the way that conditions are specified and that activities are represented.

The same linds of elements mentioned as initiators can be used as terms in the definition of a condition for a $K S$, provided they are present in one form or another within the system (this is no restriction for situation or blackboard state, but might be a restriction, e.g., for goals). The possible variation for specifying conditions lies in the specific kinds of 
elements that can be used as well as in the allowed combinations. As an illustration: many blackboard architectures allow arbitrarily complex combinations of predicates over the blackboard state, but evaluating combinations of events (let alone combinations of events that have occurred during different stages in the problem-solving process) is much less common.

Dynamic definition of the condition part of KSs. The issue of a dynamic definition of the condition part of KSs is addressed in the following section on the flow of control in blackboard architectures.

\section{Action part}

The action parts of KSs are modular and the internal representation of activities should have no consequences, therefore, to the workings of the rest of a blackboard system. To give an idea of the variation possibilities within action parts, we note that examples exist of KS action parts containing rulles (combined with all kinds of different rule evaluators) or procedures or functions. Any knowledge-representation mechanism and inferencing technique could be used inside the action part of a $\mathrm{KS}$, including frame-based reasoning and object-oriented programming, to name a few. One of the strong points of the blackboard architecture is that different knowledge-representation mechanisms and inferencing techniques can be incorporated within one system; each KS applying the mechanism best suited for its task. If parallel hardware is available, activities within the action parts of IKSs can be executed even in parallel.

Dynamic definition of the action part of KSs. The issue of making the definition of the action part of KSs dynamic was already addressed in the discussion about making the contents of KSs available to other KSs (by placing part of KS expertise on the blackboard, which is in fact only one option).

\section{Additional variation opportunities}

Apart from the obligatory condition and action parts of a KS identified in the previous section, some additional attributes can be defined for KSs. Such attributes can be used, e.g., for binding variables in a. KS with the values defined by an initiator and selecting KSs. 
Binding. In many cases the action part of a $\mathrm{KS}$ is a procedure to be applied on certain variables. These variables form a context for a specific KS instantiation and may, e.g., be defined by the initiator of the KS (situation, event, object, or goal). Variable binding is used to link a specific initiator to the variables. A distinction can be made here between a scheme in which a KS instantiation is created for each initiator and binding occurs for just this initiator, and one where references to new relevant initiators are added to the appropriate variables for an already existing instantiation which then should, upon invocation, apply its activities to all initiators.

Reasoning about actions. As the activities in a KS need a description of when the KS is applicable, it is only a small step to include more information about that KS to be used in assessing how well its contribution fits in the problem-solving process. Especially when we have to deal with limited resources (and in fact almost all sequential implementations of the blackboard architecture have to deal with limited processing capacity), it becomes increasingly important to represent information about the tasks in a blackboard system which subsequently can be used in mediating between any number of tasks competing for resources, such as processing capacity, time, and memory space. The tasks in a blackboard system (KS instantiations) are typically pairs of a $K S$ and a corresponding initiator. Information about tasks includes therefore information about the $\mathrm{KS}$ and/or the initiator.

Figure 7.2 illustrates the possible variation in information that can be used for selecting the most appropriate activity at any time during the problem-solving process. Figure 7.2 consists of two halves to indicate that relevant selection information may be found on the blackboard (the top-left half) or in KS descriptors (the bottom-right half). The semicircles inclicate ever-increasing amounts of information that can be used for selection. The inmermost two semicircles represent information provided by the triggering unit on the blackboard and the bound variables (context) for a KS instantiation, respectively. Apart from the triggering unit, additional blackboard information might be, e.g., all information on a specific blackboard level, all information on a "control' blackboard panel, all information on the blackboard (sic), etc.. Additional information about KSs to be used for selection might be KS features, expected results (or effects) of $\mathrm{KSs}$, etc. 


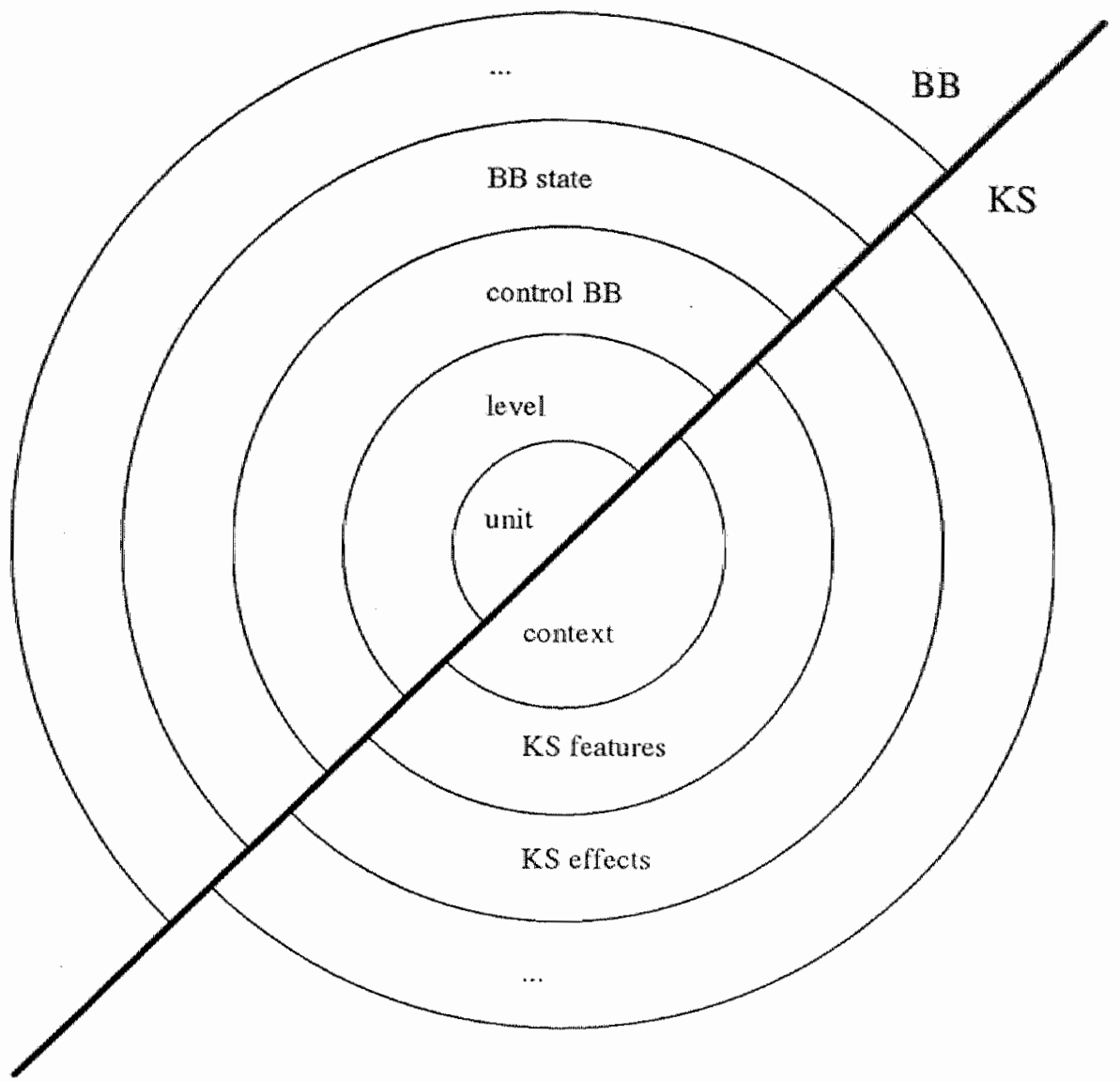

Figure 7.2: Variations in information used for selection of activities.

\subsubsection{Flow of control}

In this section we give special attention to issues on the flow of control in blackboard architectures, i.e., the central theme of matching blackboard states and blackboard state changes with knolwedge source conditions. This aspect is crucial for continuation of the problem-solving process in blackboard systems. "This discussion addresses the purpose of the flow of control, a priori defined variation and runtime variation, KSI life cycles, and 'immediate actions'. 


\section{Purpose of flow of control}

Without an adequate mechanism for identifying when $\mathrm{KSs}$ can contribute to the problem-solving process, there is no process at all. In Section 7.2.2 we have identified four different kinds of initiator for a task: situation, event, object, and goal. Initiators are not always sufficiently precise to be used for specifying applicability of a KS completely. Thus, a distinction often is made between a KS for which an appropriate initiator (usually an event) has occurred and a KS which is indeed applicable. Such a division in states is quite notable in, e.g., BB1, which recognises triggered KS instantiations (when an event has occurred in which the KS is interested) and invocable KS instantiations (for which an extra "precondition" has evaluated to be true). We call the part of the condition of a KS specifying the initiators (typically the events) of interest to the KS the trigger. The part of the condition that further specifies applicability is called the precondition (see also the definitions in Chapter 6). Following these observations and definitions, the purpose to be fulfilled by the flow of control is to match the trigger of a KS with occurring initiators and (if appropriate) the precondition with other information available within the system.

\section{Variation}

Let us consider the variation of matching triggers and preconditions. Precondition matching can become quite complex and unrestricted. We limit ourselves with respect to matching of preconditions by referring to the general discussion of matching on page 204. We recognise the following dimensions for variation in matching of triggers: abstraction and granularity; composition; kind; and event-based versus KS-based mettching.

Abstraction and granularity. Variation along this dimension has at its extremes perfect matching and completely indiscriminate matching. Additionally, we distinguish token matching. Token matching is most often encountered in contexts in which only 'meaningful' initiators are represented (for example meaningful events) and are referred to by a special name, or token. Again, we refer to page 204 for a description of matching variation. 
Composition. Most blackboard architectures restrict facilities for specifying complexity in triggers to matching only elementary initiators. However, allowing compositions of elementary initiators (of the same type, e.g., combinations of events, or of different types, e.g., combinations of events and goals) can produce a useful extension. In Blondie-III we implemented and successfully used a mechanism allowing matching triggers with composite events, combined via the constructors $\mathrm{AND}, \mathrm{OR}$, and FOLLOWED-BY. Increasing the sophistication of trigger matching may be used to decrease the amount of processing involved in precondition matching.

Kind. Kind is used here, not only to indicate the type of initiator, but also to differentiate between different forms within a certain type of initiator. An example of this last differentiation is distinguishing between events specifying creation, modification, or deletion of entities on the blackboard.

Event-based matching versus KS-based matching. Most blackboard architectures take the events generated over a period of time and match these with the triggers of KSs in the system. This event-based matching is opposed to a $K S$-based mechanism, where periodically each KS is checked whether there are interesting events among the events occurred since the last time. This last approach provides a means to check also for the absence of specified events, which in some cases can be meaningful, especially in relation with a notion of time, deadlines, and error detection.

\section{Runtime variation}

Although not common in blackboard architectures, interesting properties can be ascribed to blackboard systems that allow for a dynamic definition of (parts of) the condition of KSs. A dynamic trigger definition might be useful in several respects. One form was employed previously in HASP/SIAP. When a KS in HASP/SIAP decides that not enough information is available to make a certain inference, a problem can be defined which specifies the missing data and the activities to perform when the missing data becomes available. This mechanism can be used as a form of self-suspension. In general, a dynamic definition of triggers allows $\mathbb{K S}$ s to determine opportunities for certain activities: its own as 
well as activities of other KSs. Dynamic specification or adjustment of conditions (triggers and preconditions) can be used, for instance, for increasing and decreasing thresholds and for tuning a condition as more experience is gained during the problem-solving process regarding the suitability of specific activities.

\section{KSI life cycles}

In the presentation of a formal description for blackboard architectures in Chapter 6 a description was given of the possible life cycles a $\mathrm{KS}$ instantiation (or KS, when no instantiations are distinguished). Section 6.6 also contained a presentation of the complete range of possibilities.

\section{Immediate action}

Some blackboard architectures, e.g., Hearsay-III, include a feature called immediate action. In Hearsay-III the immediate action of a $\mathrm{KS}$ is executed as soon as an instantiation of that $\mathrm{KS}$ comes into existence and is used for binding of variables and creating a data structure representing the instantiation. The enumeration of states in the life cycle of a KS as presented in Section 6.6 provides an opportunity for identifying other points where the execution of an immediate action could be meaningful. One obvious moment is when a KS becomes truly applicable (its condition is satisfied completely); this is the first moment that the availability of all information for binding is guaranteed. In most blackboard systems selection and execution occurs more or less simultaneously and execution of the action part of the KS obviates the usefulness of an immediate action. If selection and execution do not coincide, immediate action might be used to bind variables with values as they were at selection (possibly representing the arguments leading to the selection). Upon removal of a KS from the system an immediate action could be used to tidy up after that KS, for instance, to remove currently held claims to resources.

Another opportunity for a feature similar to an immediate action exists at blackboard access: blackboard entities as active values, where updating results automatically in related activities; or a demon-like imple- 
mentation of a triggering mechanism. This last option rather resembles the way in which activities are triggered in POLIGON.

\subsubsection{Control}

Contrary to the notion of flow of control, control itself (i.e., mediating between tasks competing for limited resources) is not an indispensable component of blackboard architectures. Indeed, the blackboard metaphor does not recognise control, although it can be inciuded in the form of, for instance, a discussion leader trying to regulate contributions by the specialists. In the metaphor each specialist performs a cycle (corresponding to the $K S$ cycle in our definitions) of alternately monitoring the blackboard for opportunities and executing tasks upon occurrence of such opportunities. Direct implementations of this variation do exist (Pomeroy \& Irving, 1990).

The issue of control in the blackboard architecture is in fact so complex that a special session has been dedicated to the subject during each of the first four Blackboard System Workshops. Our discussion does not attempt to address the complexity in full detail, but restricts its focus to relevant aspects of the design of a blackboard system. We address the purpose of control, location of control, selection, and execution.

\section{Purpose of control}

The primary purpose of control in a blackboard architecture is to medi. ate between KSs competing for limited resources. When a special conponent is charged with this responsibility, that same component nay be given extra functions as well, such as determining the applicability of KSs by matching conditions with information about the problem-solving state, determining the suitability of excuting a certain $\mathrm{KS}$ at a certain time, and controlling the execution of KSs.

\section{Location}

Two different 'locations' have been used for implementing control: in a dedicated control unit executing a control cycle (roughly in turn assessing applicability of $\mathrm{KSs}$, selecting $\mathrm{KSs}$, and executing $\mathrm{KSs}$ ) and in the 
form of special control $K S$ s which help decide when to apply a certain KS. Control can be implemented also as a function of the blackboard by having the blackboard preselect its most important entities and/or events before these are passed on for processing by appropriate KSs. The different approaches can, of course, be combined.

\section{Selection}

In its full complexity, the blackboard architecture makes explicit many different kinds of data that can all be used in selecting the most appropriate activity (or set of activities) at each moment during the problemsolving process. An illustration has been given in Figure 7.2. Datadriven selection uses mainly information currently present on the blackboard. Goal-directed selection is concerned more with the effects of KSs and how they fulfil pending goals. Task-oriented selection bases its decisions primarily on information about the features or characteristics of KSs.

An extra possibility for variation is the amount of information used. for selection at various stages of the problem-solving process. In realtime applications constraints due to limited resources may increase and decrease and thus it may be necessary that selection modifies its thoroughness accordingly.

\section{Execution}

Each time a task (KS or KS instantiation) is executed in fact any number of tasks could be executed: either sequentially, via time sharing (as in AGORA in case no mapping to sufficient processors exists), or concurrently (provided that there is enough processing capacity available). The possible range includes execution of one task at a time, execution of all executable tasks, or execution of a (variable) number of tasks as a group before other control activities are resumed. There exist several incentives to execute more than one task at the time. If more than one processor is available, execution of several tasks at the same time might improve efficiency. When, in certain situations, it appears that always the same sequence of activities follows, selection of activities during the execution of this sequence seems unnecessary. Selecting and executing a 
set of tasks (or macro-KSs) decreases in those cases the overhead inherent to executing a control cycle. Especially in real-time situations, such a performance increase may prove to be essential. The use of macro-KSs was described also in (Laasri et al., 1988b; Hausen-Tropper, 1989).

Parallel execution of tasks may occur either in lockstep (all task executions begin at the same time and wait for each other to end simultaneously, thus synchronising their activities) or in a more unrestricted manner. CAGE allows specification of blackboard systems proceeding according to either one of these two options. We note that one of the possible configurations of CAGE, where all executable tasks are executed without explicit synchronisation, reduces to an architecture close to the blackboard metaphor, i.e., having no control and a KS cycle instead of a. control cycle. Indeed, the behaviour of such a configuration of the CAGE architecture resembles closely POLIGON's activation behaviour.

An issue which has not yet been addressed extensively in existing blackboard architectures is interruption of the execution of KSs. In principle, KSs can be suspended from outside the blackboard system by the control component in the blackboard system, by other KSs in the system (provided that KSs can be executed concurrently), and by itself. A form of self-suspension has been implemented in HASP/SIAP. A likely reason why interruption has not been applied much is the involved complexity. When interruption is included, the following issues must be resolved:

- Requests for shared resources by the interrupted KS must be released.

- The validity of intermediate results by the interrupted KS may have become suspect, due to the interruption.

- When an interrupted $\mathrm{KS}$ is resumed, it must be able to return to its state at the time of interruption and to retain previously achieved results.

\subsection{5 'New' variations}

In the preceding sections, we have touched briefly a couple of times on possibly new options for the blackboard architecture. In this section we review some of these possibly new options explicitly. 
A number of new options can be obtained by allowing runtime definition or redefinition of aspects within a blackboard system. The following lists a number of examples.

Interactions. When a KS can reason about whether information written on the blackboard by that KS is used by other KSs, KSs might become able to tune their (indirect) interactions.

Blackboard definition. Dynamic definition of attributes, dimensions, classes or levels, etc., on the blackboard by KSs resembles the dynamic definition of a world of discourse among KSs.

Means-end analysis. By comparing the effects of KS action parts with expected results and adapting future expectations, a blackboard system can 'learn' about the usefulness of its KSs and tune its means-end assessment aspect of control.

Conditions. Dynamic definition of triggers allows KSs to determine at runtime opportunities for executing activities: their own as well as those of other KSS. Dynamic definition and adjustment of conditions (triggers and preconditions) can be used for increasing and decreasing thresholds and tuning of conditions.

Expertise. The knowledge of a $K S$ could, in theory, be represented explicitly, e.g., on the blackboard. In doing so, this expertise becomes available to other $\mathbb{K}$ Ss and, more specifically, modifiable by other KSs. Such an approach could be used to alter, improve, or externally extend KS expertise.

KS composition. Sometimes it appears that several KSs are executed in the same situation and in the same order. Combining these $\mathrm{KSs}$ in one macro-KS docreases overhead involved with selecting each KS in turn.

KS hierarchies. Issues of re-use and specialisation of expertise can be tackled by allowing hierarchies of KSs. This would give a new dimension to the inherent 'plug-ability' of the blackboard architecture.

Other interesting refinements of the blackboard architecture can be obtained by viewing the blackboard as a communications medium and implementing communication-specific features, such as: 
- unidirectional and bidirectional communication channels;

- broadcasting, multicasting, and singlecasting;

- single use and multiple use of data.

Finally, mechanisms for interruption of KS activities might be important when the blackboard architecture is used in time-critical domains.

\subsection{Distinctive features of the blackboard ar- chitecture}

We now identify the distinctive features of the blackboard architecture. Comparison of the blackboard architecture with other computational frameworks, such as rule-based programming and object-oriented programming, shows that the blackboard architecture may share some characteristics with such other frameworks. Yet, the blackboard architecture has a set of characteristics which together set it apart from other frameworks and define the possibility and desirability of application of the blackboard architecture in occurring situations.

In this section we first discuss briefly similarities and dissimilarities between the blackboard architecture on the one hand and rule-based programming and object-oriented programming on the other hand and obtain our first hints towards identification of distinctive features. We then observe which commonalities exist for the fourteen blackboard systems examined in this chapter to find architectural distinctive features. Finally, we discuss other, behavioural, features of the blackboard architecture to complete our identification of distinctive features.

\subsubsection{Comparison with other computational frameworks}

We now compare the blackboard architecture with rule-based programming and with object-oriented programming. The discussion is phrased in general terms, as the boundaries between two frameworks are sometimes vague. Examples are imaginable of systems combining aspects of two frameworks, such as an object-oriented program using a blackboard 
for communication or a "blackboard system", where each KS consists of only one rule.

If one compares blackboard systems with rule-based expert systems, the following similarity is obvious. Knowledge sources can be viewed as (possibly large and complex) rules with a condition part and an action part. Moreover, a blackboard resembles the working space in a rulebased system, while a control unit has a similar responsibility as a rulefiring mechanism.

However, there exist some clear distinctions as well. The working space is mainly an unstructured collection of data, while ordinarily a blackboard is structured highly. Further, the procedure for selecting the next activity is typically more 'knowledge based' in blackboard systems than in rule-based systems: one can use triggering mechanisms and an explicit representation of the state of the problem-solving process on the blackboard as well as information about the activities themselves.

Another comparison involves the blackboard architecture and objectoriented programming. Both paradigms are basically modular (KSs in blackboard systems; objects in object-oriented systems) and both provide an explicit framework for communication between the modules (using creation, modification, and removal of data on the blackboard and using messages in object-oriented systems). We note, however, that messages in object-oriented systems are sent to objects directly, while KSs do not address each other directly. Another important difference is the usual absence in object-oriented systems of priorities or any other type of global control. Moreover, almost all blackboard systems lack the possibility of defining hierarchies between $\mathrm{KSs}$, thus exploiting inheritance and reuse of code.

Some of these differences seem important in deciding whether a given system has a blackboard architecture:

- the existence of a memory space accessible by different modules (KSs) and the ability to structure this memory according to generic aspects of the problem-solving process;

- the ability to make well-reasoned (knowledge-based) decisions on a. solution path based on already achieved intermediate results as well as to decide dynamically on a problem-solving strategy (opportunistic problem solving). 
Indeed, these aspects prove to be part of the commonalities that can be observed for the examined blackboard architectures, as we show in the following section.

\subsubsection{Architectural features}

We now proceed by examining the architectural commonalities for the fourteen blackboard architectures. Although implementations of common aspects may vary greatly, the following architectural features are present in all examined blackboard architectures.

Blackboard. Evidently, each blackboard system contains a blackboard for the exchange of data between the processing components (KSs) of the system.

Blackboard structuring. The blackboards in all blackboard systems have been structured to bring together data which is similar along some dimension (not necessarily abstraction in the problem-solving process).

Determining applicability. All blackboard systems contain a mechanism for assessing applicability of its activities (KSs), based primarily on blackboard-state changes (and possibly on blackboard states as well). Blackboard changes (or events) form the essential ingredient for a blackboard system to proceed and could be seen therefore as fuel for the problem-solving process.

Modularity. Blackboard architectures are all strictly modular. All activities not executed by a controlling component are collected in KSs. The allowed blackboard interactions completely define the possible interfaces for the action parts of the KSs. A blackboard architecture also provides a generic mechanism for deciding applicability of KSs (instantiations) and for executing them.

Granularity of KSs. In all examined blackboard applications, the KSs contain a number of activities. Thereby, the granularity of KSs is coarser than that of, for instance, rules in rule-based systems. Coarse granularity is consistent with the idea of KSs representing bodies of expertise, as evident in the blackboard metaphor. 
These are the architectural features which in our view distinguish blackboard architectures from other computational frameworks. However, as we have described formally, blackboard architectures only, a final word on this should be postponed until similar studies have been performed for other computational frameworks as well.

\subsubsection{Behavioural characteristics}

Let us now examine some important behavioural characteristics of blackboard architectures emerging from the architectural features.

An important aspect of the original definition of the architecture for the speech understanding system Hearsay-II was that it could be used in a natural way in a hypothesize-and-test paradigm (Reddy et al., 1973). We note that this is an important aspect of blackboard architectures in general. The blackboard should not be seen as merely a mechanism for communication. Entities placed on the blackboard are made available for inspection, validation, and improvement by other KSs. According to this view, an architecture like AGORA should not be termed a "blackboard architecture", as there the so-called entity cliques have a functionality restricted to communication only and do not allow modification of once created entities.

As a consequence of this view, the blackboard as a tool in a hypothesizeand-test paradigm provides another important characteristic: its ability to process and develop alternative (possibly even competing) partial results or hypotheses simultaneously.

The asynchronous nature of communication via a blackboard leads us to another characteristic of blackboard architectures. Results of previously executed activities (KSs) remain present on the blackboard and available to other KSs untill those results are removed explicitly from the blackboard. In order to achieve a similar feature, termed latency by Corkill (1987), programs entirely based on some procedural programming language would need to use many global variables.

The strict modularity of blackboard architectures makes them especially useful for applications where components of a system need to be modified, added, or removed as the system evolves according to changing requirements: KSs can be plugged into and out of a blackboard system in a fairly easy manner (although probably some attention would need 
to be paid to the flow of control). Not surprisingly, the blackboard architecture has been applied successfully in research applications where understanding of the problem domain increased with the development of the system.

It was shown in (McCracken, 1979) that the modularity provided by the KSs can increase efficiency in an application, due to the localisation of portions of working memory and control within KSs not obtained when the same knowledge and functionality could have been implemented as a single rule base. Thus, localisation of memory and control is an important characteristic of the blackboard architecture, stemming from the modularity of the architecture.

\subsection{Evaluation}

In this chapter we supplied answers to the four basic questions posed in Chapter 1. Based on formal descriptions of a collection of fourteen existing blackboard architectures we have identified in Section 7.1 exist. ing architectural options and variations in those systems. Although this presents not a complete overview of all existing options, the fourteen architectures were selected such that an important subset was obtained.

The inventory of existing options and variations in Section 7.2 led to a listing of the complete range of options and variations for blackboard architectures. Although it is impossible to identify any of those options safely as new, innovations of the blackboard architecture can be found especially in the area of allowing dynamic definitions of certain aspects of a blackboard system. By maling definitions of components in the blackboard architecture dynamic, the architecture can be made adaptive or, possibly, be given learning capabilities. Examples of opportunities for developing "new" options in blackboard architectures were presented in Section 7.2.5. The discussion of semantics of each option also provided in Section 7.2 can be instrumental in deciling which options to choose in order to obtain an architecture with certain desired characteristics.

Listing the variation in existing blackboard architectures in this chapter also formed a means for addressing the features which distinguish blackboard architectures from other computational rrameworks as well 
as defining the behavioural characteristics which determine the applicability of the blackboard architecture to certain application domains. This identification of distinctive features was presented in Section 7.3.

Finally, we conclude by observing that it proved to be possible, as hoped, to provide answers to our four basic questions by performing a formal study of the blackboard architecture. This suggests that analogous studies of other computational frameworks might increase understanding of those frameworks as well. 


\section{Chapter 8}

\section{A Proposal for Integration of Goal-directed Control}

In Section 2.3 a discussion of blackboard control and terminology has been presented. Among the terms explained were data-driven and eventdriven control as well as goal-directed control. It was also explained that the blackboard architecture is mostly a data-driven and/or eventdriven architecture: a KS monitors the blackboard for occurrences of blackboard data that enable execution of this KS or even make execution desirable. By implementing this model, using blackboard changes or events to trigger the execution of $\mathrm{KSs}$, an event-driven architecture is obtained.

However, goal-directed control can be incorporated within a blackboard system also, as has been shown, e.g., in (Corkill et al, 1982; Johnson \& Hayes-Roth, 1986). In goal-directed control the desirability of executing a $\mathrm{KS}$ is not rated by matching conditions and other attributes of the KS with currently available data, but by matching expected effects of the KS with (sub)goals presently held within the system.

We are interested in studying ways not only to incorporate goal-directed control in a blackboard system, but also to establish integration of datadriven and goal-directed control within a blackboard system, i.e., to be able to rate KSs against currently held data and goals simultaneously. None of the previous efforts in the literature present a true integration of data-driven and goal-directed control. The aim of the research presented 
in this chapter was to design a mechanism which enables selection of a. KS (or KS instantiation) based both on the desirability of applying that KS (instantiation) on currently available data, and on the expected effectiveness of executing that $\mathrm{KS}$ (instantiation) in light of the goals currently represented within the system. The result is a proposal for such a meclianism based on Maes' spreading activation network for action selection (Maes, 1989a; Maes, 1989b).

There are several reasons for performing this study. An integration of data-driven and goau-directed control is expected to be useful in sophisticated problem solving in general. Goal-directed control can augment data-driven control, while, conversely, data-driven control may be essential to employ effectively goal-directed control, as we will explain shortly. On the one hand, without respecting the goals and the effects of activities in relation to these goals in otherwise data-driven control, problem-solving might become ineffective and ill-directed. Goals can be used to mediate between activities which might be indistinguishable when activities are selected for execution using only current state data. Furthermore, goals provide a 'direction' for building plans of activities that will lead eventually towards the desired goals.

On the other hand, inclusion of data or events in goal-directed control is useful to establish feedback, providing a means for monitoring and adjusting the problem-solving process. Goal-directed selection of which activity to perform next makes assumptions about the effects of the activities, which may turn ont to be incorrect or insufficiently detailed. The problem-solving process must therefore be able to adjust and refine its plans as deviations from expected intermediate results are observed.

Incorporation of goal-directed control and explicitly representing goals provides facilities not only for guiding problem solving in a promising direction, but also for reasoning about goals and their relations. This is for instance illustrated in (Lesser et al, 1988; Lesser et al., 1989a; Lesser el al., 1989b). Table 8.1 presents the relationships between goals described in (Lesser et al, 1.989b).

Relationships among goals can be used to avoid redundant and useless tasks, as well as to identify activities which are relevant in more than one respect. This can greatly improve the efficiency of the problem-solving task as illustrated via experiments in (Lesser et al., 1989a). 


\section{Subsumption}

Subsumption occurs when the specification of one goal completely encompasses the definition of another goal.

\section{Assistance}

The situation in which satisfaction of one goall implies satisfaction of another goal, is called assistance.

\section{Competition}

Two goals compete if there is no partial solution that can satisfy both.

\section{Cooperation}

Two goals cooperale if they produce information that possibly can be integrated into one partial result.

\section{Independence}

Two goals are independent if they are neither competing nor cooperating.

\section{Subsumption-inhibition}

In case of subsumption the subsumed goal can be inhibited to avoid redundancy.

\section{Assistance-inhibition}

When a goal assists another goal and the first goal is inhibited, assistanceinhibition inhibits the part of the second goal not encompassed by the first.

Table 8.1: Goal relationships as described in (Lesser et al, 1989b).

In this chapter, we present the results of our study of integrating datadriven and goal-directed control in blackboard systems. Section 8.1 describes and discusses other approaches from the literature. Then, in. Section 8.2, Maes' activation network is described, which forms the basis of our approach. Section 8.3 describes the adaptation of activation networks to blackboard architectures for the selection of KS instanti. ations to combine data-driven control with goal-directed information. We propose this mechanism as an approach lo integrate data-driven and goal-directed control. An evaluation of the work described in this chapter is presented in Section 8.4.

\subsection{Goal-directed control in blackboard archi- tectures}

Not many blackboard systems can be said to be completely data-driven or event-driven. The use of additional information to assess (roughly) the usefulness of activities to reach a solution does introduce some goaldirected control. In addition, important information is available implic- 
itly in the definitions of KSs: chains of possible activities are defined by the changes that one KS makes to the blackboard and which subsequently may trigger other KSs. In a well-designed blackboard system, these possible chains of KS invocations ensure that problem solving leads eventually, if indeed possible, to a solution.

Severai proposals for the incorporation of explicit goal-directed control in a blackboard system are to be found in the literature. One such proposal is presented in a retrospective view of the Hearsay-II architecture (Lesser \& Erman, 1988). This approach suggested the creation of goal nodes on the blackboard, which should be distinguishable from the hypotheses on the blackboard. By associating events with the creation of goal nodes KSs can trigger on goal nodes and process them. However, this approach does not constitute actual goal-directed processing, but implements an integration of top-down processing with bottom-up processing (which, as we have seen in Section 2.3, should not be confused with the kind of integration we are looking for).

Aiello (1983) presents a comparison of different control strategies in a blackboard application built in AGE, one of which can be identified as goal-directed. In this approach goal-directed control is associated with backward chaining of production rules. The KSs in AGE are sets of production rules. In the goal-directed version of the application, all rules are combined within one KS. In ordinary use, the antecedents of those rules are matched with available data. During goal-directed processing, however, the blackboard is used for explicitly representing goals. The consequents of the production rules in the KSs are matched with these goals. Although the efficiency of this approach did not compare favourably with the two other approaches in the same study (implementing event-driven and expectation-driven, or model-driven, control), this shortcoming can be attributed in part to inefficiencies introduced by implementing the application as a single $\mathrm{KS}$.

The blackboard shell MXA for signal interpretation tasks (Stammers, 1985; Tailor, 1988) also provides backward chaining within KSs as a means for incorporating goal-directed processing.

In the following sections, we describe and discuss more extensively two distinct approaches for incorporating goal-directed control in blackboard architectures. 


\subsubsection{Goal-directed control in BB1}

Johnson and Hayes-Roth (1986) describe an approach for incorporating goal-directed control within the control architecture of BB1. This approach is based on the following observation:

"Goal-directed reasoning entails identifying and performing actions in order to perform other desirable actions. These other actions may be desirable per se or because of their effects." (Johnson \& Hayes-Roth, 1986, p. 6)

This observation implies two different situations in which can occur goaldirected selection of activities:

- the existence of an important subgoal (called focus in BB1), but no executable KSARs to satisfy this goal,

- the existence of a highly-rated, but nonexecutable KSAR.

The task of goal-directed KSAR selection is to find and execute KSARs resolving such a situation. If one of the thus selected KSARs is not yet executable itself, goal-directed control can proceed recursively to find and execute KSARs that will make the appropriate KSARs executable. Finding and executing a IKSAR in BB 1 involves knowing which KSAR will (probably) have the desired effects, i.e., satisfy a certain goal or create an event that will trigger a suitable KSAR.

If at any time in the problem-solving process no invocable KSAR is avallable that rates highly against some (important) focus, a special, domain independent, control KS is triggered. This KS, called SatisfyPriority-Focus, determines which (possibly nonexecutable) KSs would rate highly against the focus. When there exists a triggered, but not yet invocable, KSAR for one of the potential KSs, Satisfy-Priority-Focus posts another focus on the blackboard for each false condition for that ISSAR, urging the satisfaction of a false condition. If there is not even a triggered KSAR available that might satisfy the focus, a focus is created on the blackboard stimulating the creation of an event that will trigger a promising KS. Invocable KSARs rating highly against these new focj are then selected by the control unit of $B B 1$. If their activities result in satisfying the original focus, another control KS, Teminate-Prescription, is 
triggered which will deactivate the goal-directed behaviour. Otherwise the whole procedure is repeated recursively.

BB1 triggers another generic control KS, called Enable-Priority-Action, to resolve situations where there exist highly-rated, but nonexecutable KSARs. BB1 then proceeds as in the previous case.

\section{Discussion}

As seen in the description above, goal-directed control is implemented using control KSs. Although such a uniform approach helps to clarify the structure of the problem-solving process, it also introduces the significant overhead of executing a complete control cycle for each control KS. Implementing goal-directed control as part of the control cycle would increase the cost of executing a single cycle, but might reduce the total overhead during the complete problem-solving process.

Moreover, the implementation of goal-directed control by Johnson and Hayes-Roth does not really provide an integration of goal-directed and data-driven control: goal-directed control is enabled by the triggering of the KS Satisfy-Priority-Focus and subsequently disabled by the KS Terminate-Prescription, after which control proceeds again in a datadriven manner. Thus, goal-directed and data-driven control are employed alternately.

\subsubsection{Goal-directed control in DVMT}

Another approach to incorporate goal-directed control in a blackboard architecture is described in (Corkill et al, 1982). This approach added goal-directed control to the architecture of Hearsay-II (Erman et al, 1980). The new architecture was then used as a building block in the distributed problem-solving network DVMT (Distributed Vehicle Monitoring Testbed; Lesser \& Corkill, 1983). The following observation guides this approach:

"The stimulation of a precondition process in the data-directed architecture not only indicates that it may be possible to execute the knowledge source, but that it may be desirable 
to do so in order to achieve the goal implicit in the output of the KS." (Corkill et al., 1982, p. 145)

The stimulation of a precondition process (which occurs when a trigger matches an event) is used normally to indicate that (part of) the condition for execution of a KS is fulfilled and that it is (almost) possible to execute a KS. However, Corkill et al. state that the triggering of a KS also signals that a KS should be executed as the event marks a situation in which it is expedient to execute the triggered $\mathrm{KS}$.

A precondition process in Hearsay-II involves a mapping from events to KSs: whenever an event occurs, this event is matched with the preconditions of the KSs to find which KSs are triggered. In DVMT, this mapping is split into two separate mappings: from events to goals and from goals to KSs. Based on the above observation, it is felt that the occurrence of events should be used to identify goals for the problemsolving process.

DVMT contains a separate blackboard, termed the goal blackboard. The dimensionality of this goal blackboard mirrors the original blackboard (called data blackboard): an item on the goal blackboard is a goal, representing a request to create an item on the corresponding area on the data blackboard. The mapping of events onto goals is used to determine which goals should be added to the goal blackboard. External sources such as KSs may also place goals on the goal blackboard. This last feature can be used to direct problem solving over a period of time.

Control in DVMT is further extended with a component called the planner. This planner uses mappings of goals onto KSs to select KSs that can satisfy the goals on the goal blackboard. This may involve selecting other KSs recursively to satisfy preconditions of already selected, but nonexecutable KSs. If no KSs exist that can satisfy a goal completely, the plamner tries to decompose the goal into subgoals, which are then treated anălogously.

\section{Discussion}

The approach as described above makes explicit several aspects of goaldirected control. The transparency thus achieved can be used to improve efficiency of the problem-solving process (Lesser et al., 1989a). Since 
goal-directed control in DVMT is included in the control cycle, this approach reduces the overhead inherent in using specialised $\mathrm{KSs}$ for control tasks, which is the approach taken in BB1.

Yet, the underlying assumption that goals can be generated from events seems to be at least counterintuitive: mapping events onto goals suggests that activities just performed indicate which direction to take next. This is not the case in general. Imagine, e.g., the case in which the same type of events occur while trying to solve completely different problems within the same problem domain. Although an already taken path indicates some sense of direction, it can hardly be decisive in selecting the right continuation at any given moment during the problem-solving process, as it does not contain information about the alternatives between which to choose.

Moreover, the event-to-goal mapping translates events into goals instead of adding goals to the information provided by data and data changes on the blackboard, thus losing the information provided by the occurrence of the events per se. It is the possibility for KSs to generate goals explicitly and to post these on the goal blackboard for inclusion in the KS selection process which provides an actual extension towards goaldirected control.

These considerations suggest that goals are better generated independently. Durfee (1988) describes one way to achieve this in domains, where no a priori enumeration of goals can be given. He noted that, although problem-solving efficiency might increase significantly by employing goal-directed control, some problem domains do not allow the explicit statement of goals at the outset of a problem-solving task. One of his examples is the recognition of objects in an image. The number of possible objects might be so large that matching parts of the image against these objects is not practical, prohibiting simple enumeration of goals. After some data-driven processing, however, enough information might be present to formulate one or more tentative goals. The process of extracting goals from partial results derived through data-driven processing, by investigating clusters of partial results with similar values for common attributes, is described in (Durfee, 1988, Chapter 3). The identified goals are then processed using goal-directed control as described a.bove. 


\subsubsection{General requirements for integration}

Current problem-solving state data as well as goals must be considered simultaneously for selecting the most desirable next KS or KS instantiation for execution, when a true integration of data-driven and goaldirected control in a blackboard system is to be realized. State data are related to the conditions of activities, while goals are related to the effects of activities. Hence, data-driven control favours activities that process highly rated state data and goal-directed control favours activities having highly-rated effects or results. Results can be rated highly when they:

- instantiate important activities,

- satisfy conditions of highly-rated activities, or

- constitute important data in their own right.

Actions themselves (possibly not yet applicable or even not yet instantiated) are rated according to their expected results, and according to the blackboard data they are supposed to process.

From these observations the following requirements can be formulated. A mechanism for the true integration of data-driven and goal-directed control must be able to represent explicitly and to reason about:

- the data and their importance,

- the relationships between data and conditions,

- the goals and their importance,

- the relationships between goals and activities,

- the relationships between activities and conditions.

All relevant state data in a blackboard system generated by knowledge sources are available on the blackboard. Analogously, relevant input to the overall system should be stored on the blackboard as well. Goals can be given as input also and can be generated during the problemsolving process as well. The generation of goals and subgoals during 
the problem-solving process is usually a domain dependent, knowledgeintensive task and should therefore be a task for the knowledge sources in the system. The blackboard should be used as a means for representing and communicating goals and subgoals.

We would like to close this section with a warning. Even when a very sophisticated control mechanism is developed, in the end the selection of a single task for execution involves comparing diverse activities using completely different arguments to assess the desirability executing these activities: it is like comparing apples and oranges. It is hard to predict and to verify the outcome of such evaluations. Therefore, tuning is inevitable and it is imperative that a selection mechanism is adaptable and allows for such tuning.

\subsection{Maes' spreading activation network}

Maes (1989a; 1989b) describes an approach to action selection which, at least at first glance, seems to provide a means for meeting the requirements as specified in Section 8.1.3. Hence, we decided to investigate this approach further. Eventually, the investigation led to the design of a mechanism for integrated data-driven and goal-directed KS selection in blackboard systems. This section describes Maes' approach, called a spreading activation network, in some detail, in so far as it is relevant to our own design.

\subsubsection{General description}

The spreading activation network is developed for the task of action selection in autonomous agents. The nodes in an activation network are called competence nodules and resemble operators in classical planning systems. The competence modules represent the actions that can be executed by the agent at one time or another. A competence module $i$ can be described by the tuple $\left(c_{i}, a_{i}, d_{i}, \alpha_{i}\right)$. Here $c_{i}$ denotes a list of preconditions which must be fulfilled before this competence module can become executable. $a_{i}$ and $d_{i}$ represent an add list and delete hist, respectively. An add list specifies the facts that will be true as a result of executing the corresponding action. A delete list specifies what will not be true anymore. Finally, $\alpha_{i}$ represents an activation level of the 
competence module $i$. The elements in the condition, add, and delete lists are propositions, not predicates. Therefore, the list elements do not contain unifiable variables. Maes does not indicate why she refrained from using unifiable predicates.

A competence module is executable when all preconditions in its condition list are fulfilled. Evidently, this means that more than one competence module can be executable at the same time. Each competence module has associated with it an activation level. Activation levels are used to select one of the executable competence modules for execution. Activation levels are determined in a similar way to activation levels in artificial neural networks are determined (Rumelhart \& McClelland, 1986). Competence modules are linked together and activation is spread through the resulting network (called the activation network) according to input from the environment, the nature of the links, and previous activation levels.

Maes distinguishes three different types of links: successor links, predecessor links, and conflictor links. These links can be compiled from the condition lists, add lists, and delete lists of the competence modules:

- There is a successor link from competence module $x$ to competence module $y$ for every proposition $P$ that is a member of the add list of $x$ and also a member of the precondition list of $y$.

- Conversely, there is a predecessor link from module $y$ to module $x$ for every successor link from $x$ to $y$.

- A conflictor link exists from module $x$ to module $y$ for every proposition $P$ that is a member of the precondition list of $x$ and a member of the delete list of $y$.

Within an activation network, competence modules can influence each other's activation levels (or simply activation) via activation spreading over these links. Executable competence modules increase activation of all successors and decrease activation of (inhibit) all conflicting modules. Currently nonexecutable modules increase the activation of predecessors. This way, important competence modules (competence modules with a high activation level) that are not yet executable can increase the activation levels of predecessors in order to increase the chance of a speedy execution of competence modules that enable the execution of 
the important module. In order to avoid the complete annihilation of two mutually inhibiting modules, only a module with a higher activation level can inhibit another module (which also prevents inhibition by a module of itself).

Activation of competence modules within the activation network can also be influenced from outside the network in the following ways.

Activation by the current state. The activation of a module is increased when a proposition in its condition list is true in the current state of the environment of the agent.

Activation by goals. The activation of a module is increased when one of the agent's (not yet achieved) goals is matched by a proposition in the add list of that module.

Inhibition by achieved goals. Activation of a module is decreased when a proposition in the delete list of that module matches an already achieved goal.

\subsubsection{Maes' algorithm}

The agent will select actions for execution following an algorithm in which activation levels are computed and spread through the network. This algorithm is used in a loop, while in each step the described computation is performed for all competence modules.

1. The impact of the current state of the environment and the goals on the activation of competence modules is computed. By repeating this step during each cycle, the algorithm can deal with (sudden) changes of both state and goals.

2. Activations and inhibitions of modules through successor, predecessor, and conflictor links are computed.

3. A decay function is used to ensure that the overall activation level remains more or less constant.

4. The competence module that wins in a competition where each module tries to acquire the highest activation level, i.e., the module that fulfils the following three conditions, is selected for execution: 
(a) it is executable,

(b) its level of activation is higher than a certain threshold,

(c) it has a higher level of activation than all others that fulfil the previous two conditions.

In case more than one module satisfies these conditions, one of those modules is chosen (arbitrarily). After a module has become active, its activation level is reset to zero.

As a result of the inputs from state information and goal information and the activation spreading behaviour as explained earlier, an activation network is capable of exhibiting both data-driven and goal-directed action selection at the same time. Goals influence activation levels of competence modules over predecessor links in such a way that information about these goals and ways to achieve them can be combined with information about the current state in order to select the 'best' next action.

\subsubsection{Global characteristics}

Maes states that a network as described above can be modelled by a system of differential equations, but solving this system is too complex for present-day system theory. However, a number of interesting qualitative statements are obtained. They have been arrived at either by empirical studies or by close examination of the network. This section discusses some of these statements. Maes recognises four global parameters for tuning the dynamics of an activation network. These global parameters are used in the following discussion.

- $\theta$ is the threshold for becoming active. The value of $\theta$ is lowered every time none of the competence modules could be selected for execution.

- $\sigma$ is the amount of activation added to the activation level of a competence module for each proposition in its condition list observed to be true in the current state. 
- $\gamma$ is the amount of activation added to the activation level of a module for each proposition in its add list which matches a current goal.

- $\delta$ is the amount of activation subtracted from the activation level of a module for each proposition in its delete list which matches an achieved goal.

Figures 8.1 and 8.2 illustrate the roles of these global parameters. In Figure 8.1 the role of $\theta$ is shown. The rectangles represent the activation levels of competence modules (black rectangles correspond with executable modules). The algorithm for calculating activation levels executes a loop. The activation levels of the same modules are shown as calculated during three subsequent cycles of the loop. Each time the threshold $\theta$ (shown as a dashed line) is lowered until a module can be selected for execution. At time $t=2$, one module has an activation level greater than $\theta$ and is selected.

The parameters $\sigma, \gamma$, and $\delta$ are illustrated in Figure 8.2. The circle represents a node. The arrows at the bottom of the circle correspond to elements in the condition list (a dashed line indicates a condition currently not fulfilled). The arrows at the top of the circle correspond with elements in the action list and the delete list. An arrow pointing towards the circle inclicates an element in the action list; otherwise an element in the delete list is indicated. Here, a dashed line represents an element which does not match a current goal. A new activation level for the node in this specific example is calculated by taking the old activation level and adding $\sigma$ twice, adding $\gamma$ once, and subtracting $\delta$ once.

The parameters $\sigma$ and $\gamma$ can be used to influence the data-driven and goal-directed behaviour of an activation network. A high value for $\gamma_{3}$ for instance, will increase the effects of goals on the outcome of the task of action selection. By putting $\sigma(\gamma)$ equal to zero, the network can be made to behave completely goal-directed (data-driven).

An activation network is able to show planning capabilities as opposed to just selecting actions one at the time without apparent relation between subsequent actions. If a sequence of competence modules exists which commects the current state with one or more goals, then this sequence becomes activated through the simultaneous effects of state input and 
$\theta$
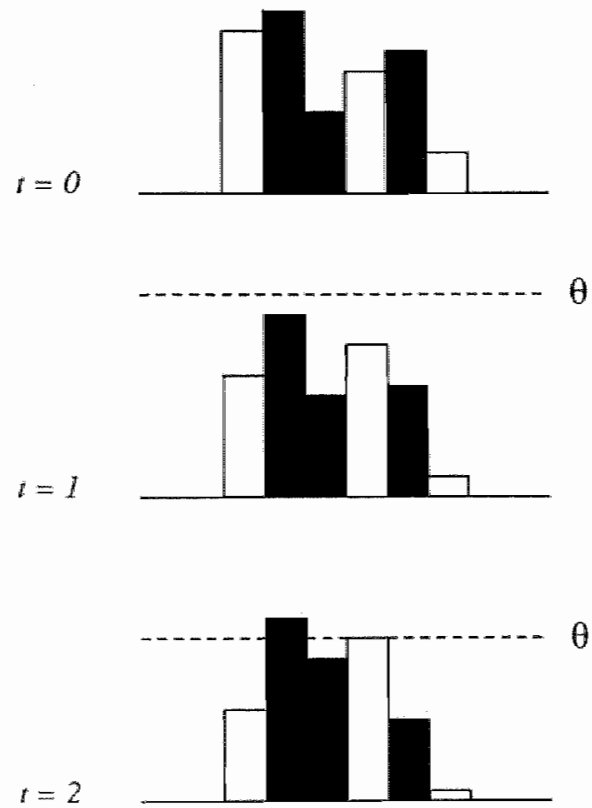

Figure 8.1: The role of $\theta$. 


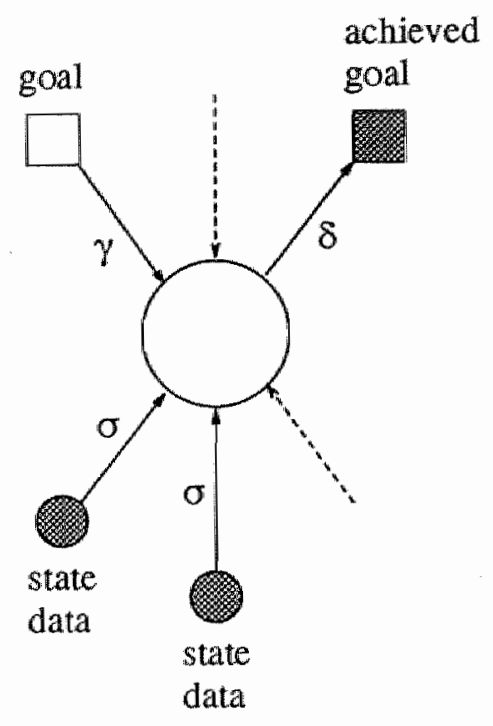

Figure 8.2: The role of $\sigma, \gamma$, and $\delta$.

goal input. Such a sequence then acts as a plan from which the network deviates only when other goals are introduced or when actually emerging state data differs from the data expected from add and delete lists.

Empirical studies show that local maxima during the selection of activities seem to be avoided, provided that the spreading of activation can continue long enough, i.e., that the threshold $\theta$ is high enough. $\theta$ indicates the level of thoughtfulness (Maes' terminology) displayed by the network. A high value for $\theta$ implies longer processing of inputs and consequently a decrease of speed. Evidently, high $\theta$ does not imply that more aspects are examined and weighed during the selection process.

The activation network appears to bias towards ongoing sequences of actions, as illustrated in Figure 8.3. The combined input of current state data and current goals will lead, typically, to high activation levels of all modules along a 'path' (a sequence of actions, drawn bold in Figure 8.3) connecting the state data and goals. Whenever the first module along this path is selected for execution, the remaining modules will still have a high activation level. This leads to the expectation that the next selected module might very well be the next module along the path. 


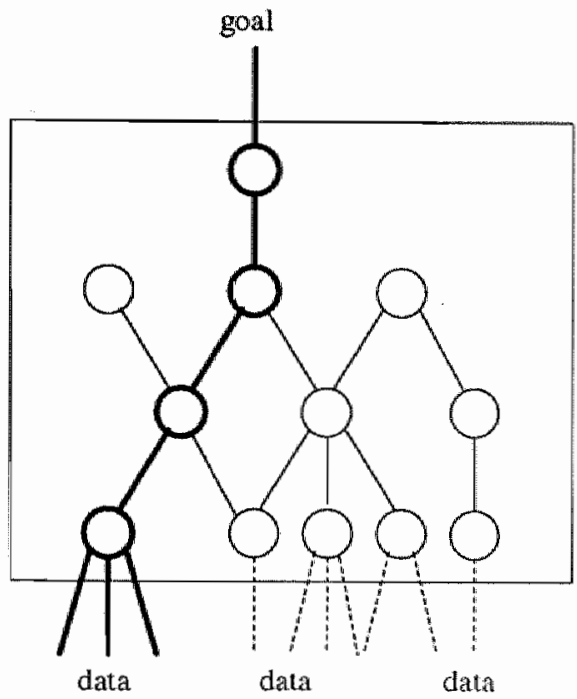

Figure 8.3: Bias towards ongoing sequences of actions.

This bias towards ongoing sequences of actions leads to a certain inertia with respect to changes in the environment (state data and/or goals). This does not mean, however, that an activation network can not react (in time) to changes in its environment. Both state and goal changes can be detected and considered in subsequent selection of activities. Adaptability is enhanced by increasing the state and goal inputs relative to internal activation spreading. Inertia is at its peak when state and goal input are neglected. So, clearly there is a trade-off between adaptability and inertia. However, the value of $\theta$ also plays a role.

By saving the activation levels of the competence modules between dif ferent selections, an activation network does not have to replan after each action. The previously found sequence of actions forms the basis for action selection in subsequent selections.

A final observation concerns situations where more than one (sub)goal exists at a certain time. Competence modules contributing to more than one (sub)goals are favoured as these modules receive activation from all of these (sub)goals. Furthermore, modules with little competition have a higher chance of being selected. 


\subsubsection{Mathematics}

In this section we supply a more detailed description of activation spreading in an activation network. For the time being we assume that no conflictor links exist ${ }^{1}$. Let the activation network consist of $n$ nodes: $N_{i}, i=1, \ldots, n$. At time $t$ the external input $E_{i}(t)$ for a node $N_{i}$ is determined by the following formula:

$$
E_{i}(t)=\sum_{j_{i}} S_{j_{i}}(t)+\sum_{k_{i}} G_{k_{i}}(t)
$$

where $j_{i}$ ranges over the propositions in the condition list of $N_{i}$ that are observed to be true at time $t ; k_{i}$ ranges over the propositions in the add list of $N_{i}$ that match current goals at time $t$. (An overview of the notation used in this section is represented in Table 8.2.) When all $S_{j_{4}}$ have an equal value, $S_{j_{i}}$ is also equal to the global parameter $\sigma$ in the previous section. Analogously, $G_{k_{i}}$ equals $\gamma$ (provided all $G_{k_{i}}$ are equal).

In order to keep global external input constant at each time point $t$ and to mediate between the relative impact of state and goal input, the following constraints are observed:

$$
\sum_{i=1}^{n} \sum_{j_{i}} S_{j_{i}}=\pi \cdot(1-\chi) \text { and } \sum_{i=1}^{n} \sum_{k_{i}} G_{k_{i}}=\pi \cdot \chi
$$

Evidently, $\sum_{i} E_{i}(t)=\pi$. If $\chi=0$, then the activation network behaves completely state-driven, while, if $\chi=1$, the activation network behaves completely goal-civen. These two constraining formulas define the acthal values of each $S_{j_{i}}$ (and $G_{k_{1}}$ ) in the following way. Initially, all $S_{j_{0}}$ are set at a certain weighing factor $w_{j_{1}}$ (in Maes' formulation all $w_{j_{k}}$ equal 1). Then the actual value $S_{j}$ is calculated as follows:

$$
S_{j_{i}}=\frac{\pi \cdot(1-x)}{\sum_{i=1}^{n_{i}} \sum_{j_{i}} w_{j_{i}}} \cdot w_{j_{i}}
$$

\footnotetext{
'When viewing knowledge sonrces or knowledge source instantiations as competence modules, in many application domains the semantics of conflictor links may lose their meaning. Add and delete lists for KSs or KSIs can be associated with their additions and deletions of data on the blackboard, respectively, while deletion of blackboard data is not often used in blackboard applications. We acldress this issne again in Secion 8.3.3.
} 
$\pi=$ the total amount of external input into the network at each time

$\varphi=$ fraction of activation energy an executable node spreads forward into the network, $\varphi \in[0,1]$

$\beta=$ fraction of activation energy a nonexecutable node spreads backward into the network, $\beta \in[0,1]$

$x=$ amount of activation energy a goal puts into the network relative to statie input, $\chi \in[0,1]$

$N_{i}=$ node $i$ of the activation network, $i=1, \ldots, n$

$S_{i}=$ state input for node $N_{i}$

$G_{i}=$ goal input for node $N_{i}$

$E_{i}=$ external input (the sum of state and goal imput) for node $N_{i}$

$B_{i}=$ backward activation for node $N_{i}$

$F_{i}=$ forward activation for node $N_{i}$

$b_{s_{i}}=$ backward activation for node $N_{i}$ from node $N_{s}$

$f_{p i}=$ forward activation for node $N_{i}$ from node $N_{p}$

$\alpha(t, t)=$ total activation of node $N_{i}$ at time $t$

$\operatorname{Ret}_{i}(t)=$ amount of activation retained by node $N_{i}$ at time $t$

$\operatorname{Rec}_{i}(t)=$ amount of activation received by node $N_{i}$ at lime $t$

Table 8.2: Glossary of notation in Maes' activation network. 
Backward and forward activation are defined as follows. Node $N_{i}$ receives input from a successor $N_{s}$ (we note that a node spreads activation backward only when its action is not executable):

$$
B_{i}(s, t)= \begin{cases}\sum_{s_{i}} b_{s_{i}}(t) & \text { if } N_{s} \text { not executable at time } t \\ 0 & \text { if } N_{s} \text { executable at time } t\end{cases}
$$

where $s_{i}$ ranges over the preconditions of node $N_{s}$ which are not true at time $t$ and which are in the add list of node $N_{\mathrm{i}}$.

Node $N_{i}$ also receives input from a predecessor $N_{p}$ (activation is spread forward by a node only when this node is executable):

$$
F_{i}(p, t)= \begin{cases}\sum_{p_{i}} f_{p_{i}}(t) & \text { if } N_{p} \text { executable at time } t \\ 0 & \text { if } N_{p} \text { not executable at time } t\end{cases}
$$

Here $p_{i}$ ranges over the add list propositions of $N_{p}$ which are not true at time $t$ and which are in the precondition list of $N_{i}$. Also $b_{s_{i}}$ and $f_{p_{i}}$ are chosen such that:

$$
\sum_{r} \sum_{s_{r}} b_{s_{r}}(t)=\alpha(s, t) \cdot \beta \text { and } \sum_{q} \sum_{q_{i}} f_{q_{i}}(t)=\alpha(p, t) \cdot \varphi
$$

where $r$ ranges over the predecessors of $N_{s}$ and $q$ ranges over the successors of $N_{p}$. The term $\alpha(j, t)$ denotes the activation level of a node $N_{j}$ at time $t . \beta$ and $\varphi$ are real numbers in the interval $[0,1]$ and are used to scale the effects of backward and forward activation spreading, respectively.

At each time point $t$ a node retains some of its own activation and possibly receives state and goal input. Furthermore, a node may receive input from other nodes. These combined inputs determine its subsequent total activation. By retaining activation, the network achieves inertia. The anount of activation which is retained by a node is related directly to the amount of activation which is spread forward or backward via successor or predecessor links, respectively. Thus for node $N_{i}$ (Figure 8.4 illustrates an activation network and activation spreading in the network): 


$$
\operatorname{Ret}_{i}(a, t)= \begin{cases}a(i, t) \cdot(1-\varphi) & \text { if } N_{i} \text { is executable and has successors } \\ a(i, t) & \text { if } N_{i} \text { is executable and } \\ & \text { has no successors } \\ a(i, t) \cdot(1-\beta) & \text { if } N_{i} \text { is not executable and } \\ a(i, t) & \text { has predecessors } \\ & \text { if } N_{i} \text { is not executable and } \\ \text { has no predecessors }\end{cases}
$$

This definition is consistent with the fact that a node spreads activation forward when it is executable and backward when it is not executable.

The input a node $N_{i}$ receives from other nodes at time $t$ is:

$$
\operatorname{Rec}_{i}(t)=\sum_{s} B_{i}(s, t)+\sum_{p} F_{i}(p, t)
$$

where $s$ ranges over all successors of $N_{i}$ and $p$ ranges over all predecessors of $N_{i}$.

Now we can give the formulas for calculating the activation of a node $N_{i}$ in the scheduling activation network ${ }^{2}$ :

$$
\begin{aligned}
& \alpha(i, 0)=0 \\
& \alpha(i, 1)=\operatorname{Ret}_{i}\left(E_{i}(1), 1\right) \\
& \alpha(i, t)=\operatorname{Ret}_{i}\left(E_{i}(t)+\alpha(i, t-1), t\right)+\operatorname{Rec}_{i}(t-1)
\end{aligned}
$$

We now present some properties of the thus described activation networks, which will be of use later on. The first three lemmas with their justification formulate how the total amount of activation within an activation network behaves under the formulas described in this section.

\footnotetext{
${ }^{2}$ These formulas differ slightly from the formulas in (Maes, 1989a): Reci $(t)$ is replaced by $\operatorname{Rec}(t-1)$. Using old activation levels for calculating new levels makes those calculations more straightforward, as all new actiwations can be calculated in any order. When new activation lewels are used for calculating other new activation levels ${ }_{\|}$those first levels must be calculated before the other levels. Hence, the order of calculations becomes significant. Furthermore, we will see in Lemma 3 that our formulation ensures that the total activation is constant under backward and forward activation spreading. Maes divides the right-hand side in Equation (8.5) by 2 to ensure that the global activation level remains almost constant. We will not address this issue further untill we have more clearly described the topology and dynamics of an activation network for KSI selection in Section 8.3.
} 


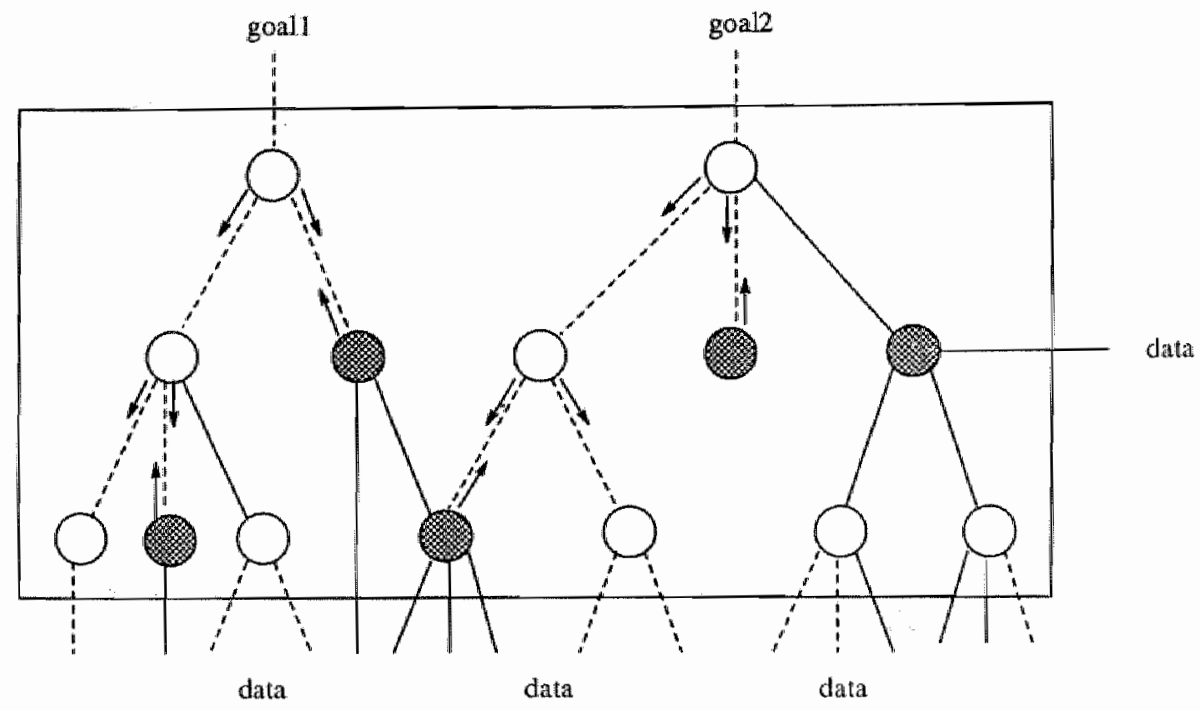

execulable module

truc propasition

(C) non-executable module

false proposition

Figure 8.4: Activation spreading in a network. The arrows indicate the direction in which competence modules send activation. 
Lemma 1 The total external input of activation into the activation network at each step of the algorithm equals $\pi$.

Justification The total input at time $t(E(t))$ into the network stems from state and goal input, i.e.,

$$
E(t)=\sum_{i}\left(\sum_{j_{i}} S_{j_{i}}(t)+\sum_{k_{i}} G_{k_{i}}(t)\right)=\pi \cdot(1-\chi)+\pi \cdot \chi=\pi
$$

Lemma 2 When a node is selected for execution, the total activation in the network drops by an amount greater than or equal to the threshold $\theta$.

Justification When a node is selected for execution, the activation of the corresponding node is reset to zero, while its activation must have been greater than or equal to $\theta$ in order to be selected.

Lemma 3 The net result of backward and forward spreading of activation in an activation network is zero.

Justification A nonexecutable node $N_{s}$ with predecessors can spread backward a total of $\alpha(s, t), \sigma$, while it retains $\alpha(s, t) \cdot(1-\beta)$. Thus no activation is lost or gained here. Equally, executable nodes that have successors can spread forward activation without adding or removing activation to or from the network. No other nodes spread activation to neighbouring nodes.

Lemmas 4, 5, and 6 provide intermediate steps for the justification of Proposition 1. In this proposition, backward propagation and forward propagation are compared. We find that these two forms of propagation are not symmetric, i.e., activation can be propagated forward through only one layer of nodes, white activation can be propagated backiward through any number of layers. In the following, $W_{t}$ denotes the propositions that are true at time $t ; e$ is a proposition which may occur as an element of a condition list or action list. 
Lemma 4 A node pair $\left(N_{p}, N_{s}\right)=\left(\left(c_{p}, a_{p}, d_{p}, \alpha_{p}\right),\left(c_{s}, a_{s}, d_{s}, \alpha_{s}\right)\right)$ interacts in backward propagation at time $t \Leftrightarrow$

1. $N_{s}$ is not executable at time $t$ and

2. $\exists e \in c_{s}: e \notin W_{t} \wedge e \in a_{p}$

Justification Immediate from the definitions.

Lemma 5 A node pair $\left(N_{p}, N_{s}\right)=\left(\left(c_{p}, a_{p}, d_{p}, \alpha_{p}\right),\left(c_{s}, a_{s}, d_{s}, \alpha_{s}\right)\right)$ interacts in forward propagation at time $t \Leftrightarrow$

1. $N_{p}$ is executable at time $t$ and

2. $\exists e \in a_{p}: e \notin W_{t} \wedge e \in c_{s}$

Justification Immediate from the definitions.

Lemma 6 If a node pair $\left(N_{p}, N_{s}\right)$ interacts in forward propagation at time $t$, then $N_{p}$ is executable at time $t$ and $N_{s}$ is not executable at time $t$. Hence forward propagation progresses through only one layer at a time.

Justification $N_{p}$ is executable follows immediately. $N_{s}$ is not executable follows from $\exists e \in a_{p}: e \notin W_{t} \wedge e \in c_{s}$.

Proposition 5 Backward propagation and forward propagation are not symmetric.

Justification From Lemma 6 , it follows that no sequence of more than one successor link $\left(\left(N_{i_{1}}, N_{i_{2}}\right),\left(N_{i_{2}}, N_{i_{3}}\right), \ldots\right)$ can exist where all links interact in forward propagation at the same time. However, for backward propagation such a sequence is quite feasible.

It is also feasible that a node interacts in both backward and forward propagation as a receiver, while it is not possible that a node interacts both ways at the same time as a sender. 


\begin{tabular}{|l|l|}
\hline Maes' activation network & blackboard architecture \\
\hline module & KS (or KSI) \\
precondition & trigger \& precondition \\
add list cq. delete list & KS descriptor slots \\
state information & blackboard data \\
goal & blackboard data \\
\hline
\end{tabular}

Table 8.3: Blackboard systems components used for implementing elements of an activation network.

\subsection{An activation network for the blackboard architecture}

In this section, we describe how the ideas of Maes" activation network can be used for the selection of knowledge sources or knowledge source instantiations in blackboard architectures. Table 8.3 displays which blackboard system components can be used for implementing elements of an activation network for KS selection. Maes uses modules, preconditions, add lists, and delete lists. We take modules to coincide with KSs. However, we have to allow for KS instantiations, which are actually KSs applied on specific data. We later discuss how to deal with KSIs in more detail.

Preconditions coincide with conditions of KSs, often implemented as triggers and preconditions in blackboard systems. Add lists and delete lists can be seen as describing the expected additions to and deletions from the blackboard by that KS and can be included in the knowledge source descriptors for use by a selection mechanism. An example of such recordings are the action-blackboard and action-level features of KS descriptors in BB1 (Hayes-Roth, 1985) and the from-level and to-level features of KS descriptors in Blondie.

The state or environment in a blackboard architecture is represented by the contents of the blackboard. Relevant items on the blackboard should therefore be used as input for the activation network for KS selection. Goals or subgoals can be represented on a separate part of the blackboard and should be used as input for the activation network in their own right. 


\subsubsection{Problems with the translation}

In this section, we focus on two problems existing for the application of an activation network to selection of activities in a blackboard system. The first problem emerges when considering KS instantiations. The second problem involves matching condition lists, add lists, and delete lists with blackboard data and goals. These problems are explained and illustrated in the following.

The activation network as described by Maes does not provide for instantiations of modules (actions as procedures applied to variables), while propositions in condition, add, and delete lists do not allow for unification. The topology of an activation network is therefore static and the number of activities represented within the network remains constant.

In most blackboard systems, however, different executable KS instantiations of the same KS may exist at the same time. Each of these KSIs represents an activity that can be selected for execution. Representation of all these KSIs as nodes in an activation network would lead to a dynamic topology for the network and a varying number of nodes. This defines the KSI problem.

KSs can be applied to different occurrences on the blackboard of the same type of event. In other words, conditions in most blackboard systems are predicates, not propositions, and allow for unification, or variable binding. Each different such binding results in a separate instantiation of the KS. (As defined in Chapter 6, a KST is a pair, consisting of the KS and the bound variables.) The need for dealing with unification of predicates defines the matching problem.

\section{KS instantiations problem}

In order to be able to distinguish KSIs during the selection task, it would seem that an activation network for KSI selection needs to include representations for instantiated KSs (illustrated in Figure 8.5). However, the number of instantiated KSs varies over time. Thus, representing KSIs as competence modules would lead to a dynamic topology for the activation network, which, in turn, would have consequences for the total activation in the network, giving raise to questions such as: do we spread the total activation over the extended set of modules; do we 


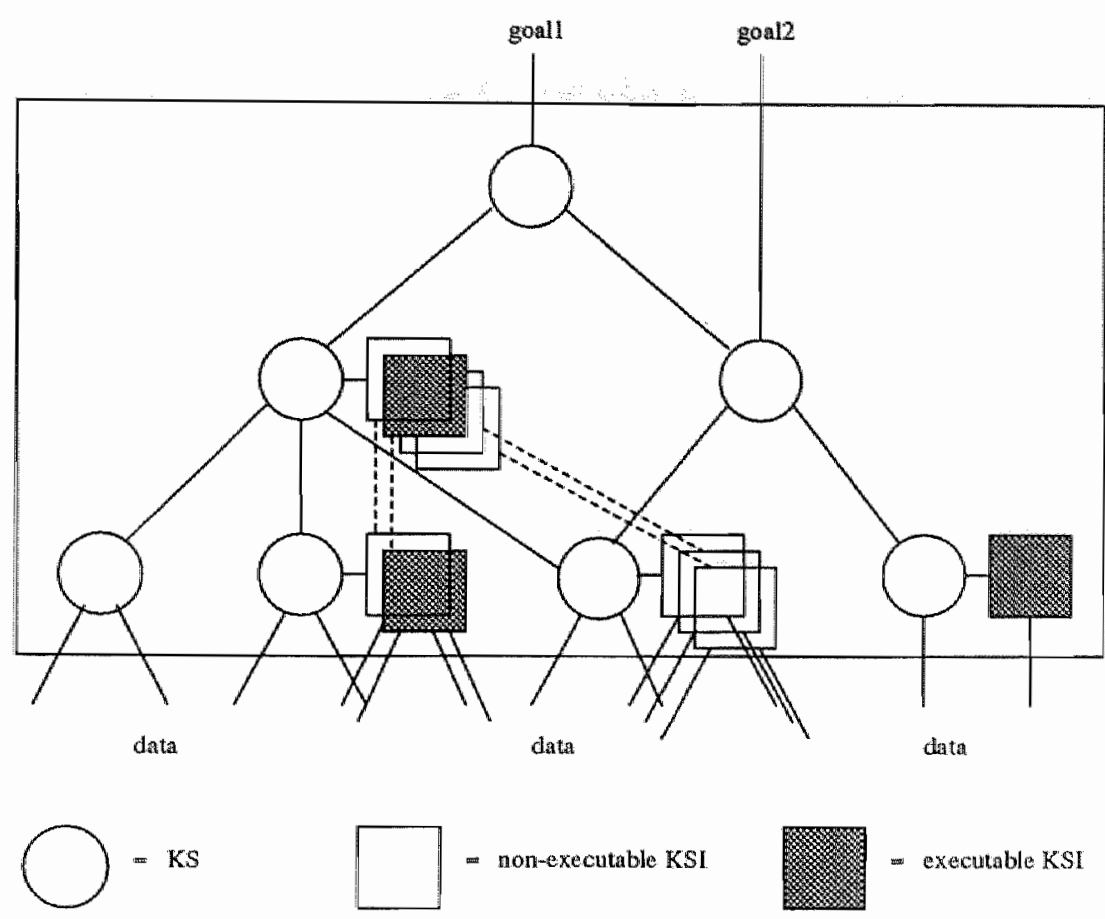

Figure 8.5: An activation network when KSIs would be included.

duplicate the activation of the corresponding KS and thus increase the total activation; do we insert a new module with zero activation and let it compete for its own activation level? The effects of either one of these approaches seem to be ill-defined at best without a careful empirical or mathematical study.

\section{Matching problem}

As seen above, predicates in the condition list may lead to the creation of different instantiations. But this is not only true for conditions. The elements in add and delete lists may lead to instantiations as well, provided that the elements in these lists are predicates. Consider the following example from a blocks world domain, see, e.g., (Winston, 1984 , pp. 35-42). Let on $(A, B)$ be a goal. The final step to reach this 
goal would be to apply the operator $\operatorname{PUTON}(X, Y)$ on blocks $A$ and $B$. $P U T O N(X, Y)$ has associated with it several conditions, including $\operatorname{clear}(X)$ and clear $(Y)$. The add list of $\operatorname{PUTON}(X, Y)$ consists of the single element $o n(X, Y)$ (and the delete list consists of the single item clear $(Y))$. PUTON $(X, Y)$ can be instantiated by unification of the add list element on $(X, Y)$ with $o n(A, B)$, as well as indirectly by unification of the condition list elements with clear $(A)$ and $\operatorname{clear}(B)$.

There are several ways in which elements in the condition and add lists can be matched and unified. Elements in the add list of a KS can be matched and unified with goals, with conditions of other KSs, and via the conditions of the KS itself. In our example: $X$ and $Y$ in the add list element $o n(X, Y)$ can be unified with the goal on $(A, B)$ or via the conditions clear $(A)$ and $\operatorname{clear}(B)$. Conversely, the elements in the condition list of a $\mathrm{KS}$ can be unified via elements in its add list as well as by unification with elements in the add and delete lists of other operators, and with data on the blackboard. In an integration of goaldirected and data-driven control all these different ways for unification may occur (six in all, see also Figure 8.6).

In many blackboard architectures, special procedures must be written to define how KS variables are bound with blackboard data upon instantiation of that KS (in Blondie binding is performed by the precondition procedure of a KS). Finding the appropriate information for binding is largely domain dependent and is thus best left to an application builder. Extending this observation to the above enumeration of ways for unification leads to the expectation that a total of four ${ }^{3}$ procedures for each KS would be needed to be able to perform all the matching and unification necessary in an integrated data-driven and goal-directed control mechanism based on activation networks. It is doubtful whether the magnitude of this additional work is acceptable in practice.

\subsubsection{Design of a blackboard activation network}

The description of the two problems in the previous section draws a rather gloomy picture of the possibility of applying activation networks

\footnotetext{
${ }^{3}$ Unification of predicates themselves within the activation network should be handled by the mechanisms implementing the activation network. Thus, unification and matching of conditions with add and delete lists of other KSs and vice versa do not need to be provided by an application builder.
} 


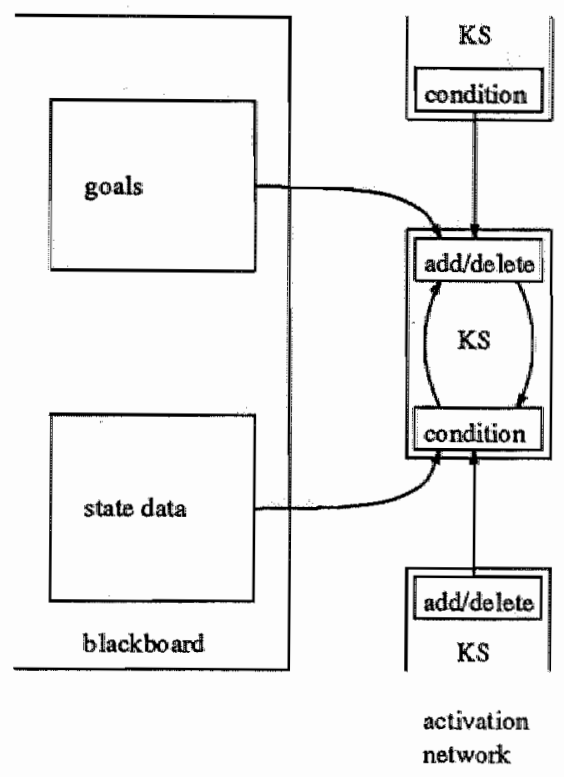

Figure 8.6: Procedures needed for matching and unification.

to KSI selection. And in fact, we do not feel that activation networks provide a good approach towards integration in its full complexity. Firstorder predicate calculus theorem provers, for instance, are designed to deal with predicates, unification of variables, and instantiations and thus should be better suited, in principle, to deal with the identified problems.

However, we show in this section that the problems we have identified can be overcome by adopting one assumption which hopefully holds in many blackboard applications. The one assumption we need is that the elements in add and delete lists must be propositions only (we do not need this assumption for condition lists). By adopting this assumption, we show that meaningful, direct links between modules representing KS instantiations (the dotted lines in Figure 8.5) can not exist, which, in turn, will help to define an activation network for KSI selection with a static topology.

As seen in the previous section, instantiations of competence modules can emerge as a result of unification of predicates in the condition lists of modules (coinciding with data-driven processing) and as a result of 
unification of predicates in the add and delete lists (coinciding with goaldirected processing). An instantiation of one $\mathrm{KS}$ can in turn give rise to the specification of instantiations of another KS, since unified predicates in one list match with predicates in lists of other KSs (these matchings are represented by the successor, predecessor, and conflictor links) leading to further unification. Eventually, this could lead to sequences of KS instantiations 'linked' through unification.

Let us now recall Lemma 6 and Proposition 5 in Section 8.2.2, stating that no sequence of more than one successor link can exist over which activation is propagated forward. The consequence is that, even if longer sequences of KS instantiations linked through unification exist, such sequences would not participate in forward propagation over sequences of more than one successor link. Thus, forward propagation of activation is restricted to isolated pairs of instantiations, only one of which is executable (and, hence, selectable). Even if competence modules were added to the activation network to represent KS instantiations, we have now seen that these KSI modules would never form a network over which activation is propagated forward.

This is not true for backward propagation. Backward propagation can very well proceed over sequences of predecessor links between (nonexecutable) modules. This means that sequences of KS instantiations linked through unification could indeed participate in backward propagation, leading to activation networks of KS instantiations in their own right. However, by prohibiting the use of predicates for add and delete lists and by allowing the use of propositions only, we can obstruct this. The result of this intervention is that the effects of different instantiations of one $\mathrm{KS}$ become indistinguishable and one instantiation is expected to contribute to the realisation of a goal or condition as well as another. Consequently, no unification can occur over predecessor links. Hence, instantiation through umification of elements in add and delete lists with goals or with elements in condition lists of other modules is made impossible, together with the corresponding backward propagation relations between instantiations. Activation as a result of backward propagation is then restricted to competence modules representing $K S s$ themselves.

The restriction regarding elements in add and delete lists has two more important consequences. Firstly, assuming this restriction, even the pairs of $\mathbb{K S}$ instantiations that can engage, in theory, in forward prop- 
agation can not be linked through unification. Secondly, prohibition of the use of predicates in the add and delete lists decreases the need for procedures for unification, since unification of conditions with elements of add and delete lists is not possible anymore. So, an application builder now only needs to specify how elements in add and delete lists match with goals, how conditions are matched with blackboard state information, and how conditions are unified with blackboard information.

Until now, we have described an approach how forward and backward propagation of activation among modules representing KS instantiations can be avoided. Still, we have to show that our simplifying assumption regarding the expressiveness of add and delete lists is not too restrictive.

One reason for assuming that using propositions instead of predicates in the add and delete lists of KSs might not be too restrictive in certain applications lies with the fact that the planning process involved in KSI selection should form an abstraction from the actual execution of KSIs. If this were not so, action selection in the problem-solving process would largely coincide with the problem-solving process itself. Thus, the contents of add and delete lists should not be a fully detailed account of what results can be expected for each input, but an abstracted representation of what generally might be expected.

Specialisation of KSs can provide a partial escape from situations where our assumption is too restrictive. In this approach the conditions of KSs are tailored to be fulfilled in only a limited number of situations, where the results can be expected to be sufficiently similar to be treated as equal by the KSI selection task.

We now take the final step and describe how to deal with $\mathrm{KS}$ instantiations in an activation network. Although we have removed all meaningful, direct relations (links in an eventual activation network) between KS instantiations, we still have to select an executable KS instantiation for execution.

We recall that under the assumption of non-unifiable elements in the add and delete lists instantiations of the same $\mathbb{K S}$ are not distinguishable with respect to their possible contributions towards the fulfilment of goals. Therefore, we have no need for an integration of goal-directed and data-driven processing to choose between instantiations of the same KS. During the selection, however, we still want to mediate between 
different instantiations based on the external input they would receive. To achieve this, we use preselection.

We propose using an activation network for KSI selection, which consists of a competence module for each KS and has connections based on the condition, add, and delete lists of these KSs. KS instantiations and their specific external inputs can participate according to the following preselection approach (see also Figure 8.7 ). When no instantiation exists for a $\mathrm{KS}$, the corresponding node in the activation network is defined as before. However, when there does exist at least one instantiation for a $\mathrm{KS}$, we first determine the executable $\mathrm{KS}$ instantiation that would receive the highest external input and use the external input of this instantiation instead of external input of the original KS to represent the KS and all its instantiations in the activation network. When there exist instantiations for a $\mathrm{KS}$, but no executable instantiations, the nonexecutable KSI with the highest external input is preselected and used into the subsequent competition within the activation network. We remark that the above distinction between cases where executable instantiations for a KS may or may not exist will be cosmetic in most cases, as usually executable KSIs will have a higher external input than nonexecutable KSIs since executable KSIs receive input from all true condition predicates.

We close this section with an observation regarding the overhead involved in using an activation network for KSI selection. The complexity of the proposed activation network is linear in the number of KSs. The most significant overhead stems from the iterative execution of a loop calculating new activation levels for the KSs. Ordinarily, it can not be determined a priori how many times this loop must be repeated before a KSI can be selected for execution.

\subsubsection{Mathematics (revised)}

We now return to the mathematics of activation networks and refine the discussion in Section 8.2.4 to propose an activation network for KSI selection. Two loose ends remained in the discussion in Section 8.2.4. The first concerns the omission of delete lists and conflictor links from the mathematical formulation. The second is tied to the desire for a constant total activation in the network. 


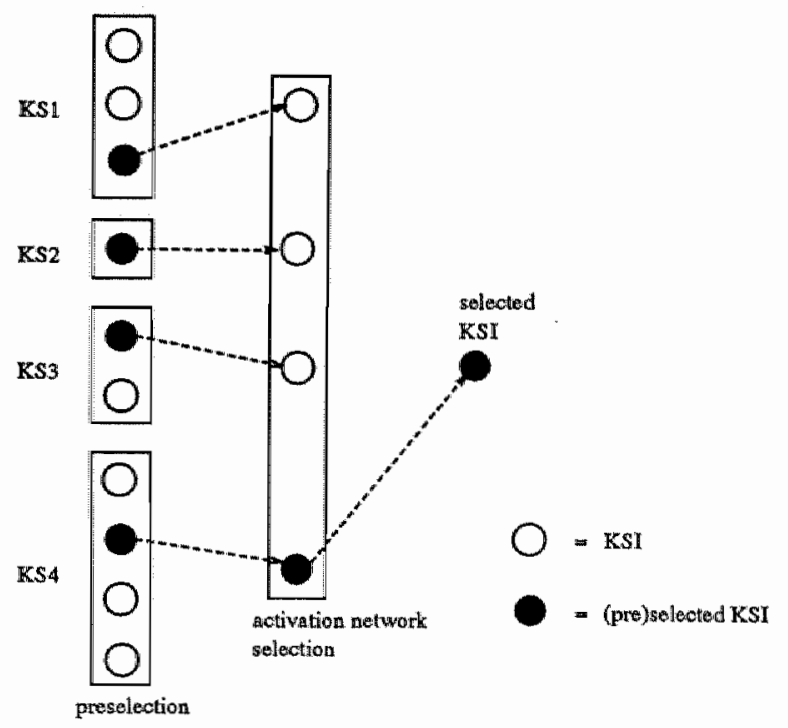

Figure 8.7: Preselection of KSIs for participation in the KSI selection by an activation network.

Delete lists are used to inhibit undesirable activities. Inhibition can be achieved by taking away activation from a competence module (see also the discussion in Section 8.2.1). Activation is taken away for each proposition in the delete list that matches with an already achieved goal and for each proposition in the delete list that matches a true predicate in the condition list of another module that has also a higher activation level.

A reason to leave inhibition out of the original discussion of mathematics is the fact that the defined decrease in activation is not compensated by an equal increase of activation in other modules. At least, there seems to be no reason to assign activation taken away from inhibited modules to specific other modules, like the ones that were responsible for the decision to take away activation. Hence, the total activation in the network is decreased as a result of inhibition, while the total activation remains constant under forward and backward spreading (Lemma 3 in Section 8.2.2). So, inhibition must be compensated in a different way. In the following we sketch a way to achieve this. 
Inhibition by already achieved goals is an external influence, but can not be treated similarly to the external input. External input was set to be equal $(=\pi)$ at all times (see Equation 8.6). If we set the total activation taken away to be constant as well, it may very well be that we need to take away more activation from inhibited modules than they actually have. Therefore, removal of activation as a result of inhibition can only be a fraction of the actual activation of inhibited modules. We calculate the total activation that is taken away from modules in this manner and spread this evenly over all modules in the network. A disadvantage of such an approach is that the effects of inhibition, which essentially belong to goal-directed processing, can not be coupled directly with $\chi$ or $\beta$, the parameters controlling the extent of goal-directed processing in the network.

Let $\delta$ be the fraction of activation that should be taken away from a module for each proposition in its delete list that either violates an already achieved goal or a true predicate in the condition list of another module. The total amount $D_{i}$ of activation taken away from module $i$ at time $t$ is:

$$
D_{i}(t)=\sum_{l_{i}=1}^{m_{i}} \delta^{l_{i}} \cdot \alpha(i, t-1)
$$

where the exponent $l_{i}$ ranges over the propositions in the delete list that match with already achieved goals or true predicates in the condition lists of other modules with an activation level greater than $\alpha(i, t-1)$ ( $m_{i}$ is the total number of such matchings). Equation 8.5 must now be adjusted to incorporate inhibition:

$$
\alpha^{\prime}(i, t)=\operatorname{Ret}_{i}\left(E_{i}(t)+\alpha(i, t-1), t\right)+\operatorname{Rec}_{i}(t-1)-D_{i}(t)
$$

We need to keep the total activation in the network constant. The Lemmas 1,2 , and 3 in Section 8.2 .2 indicate how the activation levels $\alpha^{\prime}(i, t)$ must be adjusted, except for one aspect. We have proposed to represent the external input of a KS by the highest external input possibly received by an instantiation of that. KS. When one instantiation of a KS is selected for execution, other instantiations of the same KS should not be disadvantaged during subsequent selections by a reset activation. In this light it is more appropriate to retain the same activation level and 
only adjust by exchanging the external input of the instantiation with the next highest activation.

In order to ensure a constant total activation in the network, the following amount of activation (denoted by $A(t)$ ) must be spread over the network at time $t^{4}$ :

$$
A(t)=\sum_{i}\left(D_{i}(t)-E_{i}(t)\right)
$$

This amount can be spread evenly over the competence modules in the following manner. The actual activation level $\alpha^{\prime \prime}(i, t)$ for each module will be:

$$
\alpha^{\prime \prime}(i, t)=\alpha^{\prime}(i, t)-A(t) \cdot \frac{\alpha^{\prime}(i, t)}{\sum_{i} \alpha^{\prime}(i, t)}
$$

This concludes our adaptation of Maes' activation network as a proposed mechanism for integration of goal-directed control in blackboard systems.

\subsection{Evaluation}

The work presented in this chapter was initiated by the general observation that mechanisms for integrating data-driven and goal-directed control can provide a useful extension of the typically data-driven or event-driven control in most blackboard systems. Although several examples of implementations of goal-direcled control in blackboard systems can be found in the literature, it was shown that none of these approaches provides a true integration of data-driven and goal-directed control. A possible approach for obtaining a true integration seemed to be provided by Maes' activation network. We showed how an activation network could be designed for a blackboard system in such a way that it is capable of dealing with KS instantiations (albeit in a limited way). In order to be able to adopt activation networks to KSI selection, we had to assume that the elements in add and delete lists of KSs can be expressed as propositions, not predicates. Extensions in this direction are needed, although we have argued that activation networks do not provide the appropriate technique for this.

\footnotetext{
${ }^{4}$ Of course, parameters for the network should be defined in such a way that $-A(t)$ could never be greater than the total activation in the network.
} 
During our studies we did not have available an interesting blackboard application that would also benefit sufficiently from an integration of data-driven and goal-directed control to be able to test the actual suitability of the approach for its intended purposes. Therefore, several questions remain.

Foremost, we have to concede that it is not sure that the described method provides a true, meaningful, integration of the two modes of control. It can be safely assumed from Maes' experimental results that an activation network is capable of making a data-induced selection of KS instantiations which is 'coloured' by goal-related effects. But it is not clear, without experimental results, that this colouring is in fact a meaningful and consistent implementation of integrated data-driven and goal-directed control.

Sophisticated control in blackboard systems typically uses mechanisms during the problem-solving process such as phases (Hearsay-II, Lesser \& Erman, 1988, p. 103), foci, policies, and strategies (BB1, Hayes-Roth, 1985). Experiments would be needed to determine whether such mechanisms could be used in a KSI selection mechanism based on an activation network. Also interesting would be to study how the use of (statically defined) weights to represent weak or strong relationships between competence modules in an activation network would effect the behaviour of the network and whether weights can be used to achieve useful biases towards certain relationships represented within the network.

Finally, experiments are needed to assess the computational burden of activation networks for KSI selection. An activation network will retain the activations calculated during the previous selection of a KSI and uses this for more efficient subsequent selection than would be possible when starting completely anew each time. Still, these calculations may be quite extensive. The actual cost of this and how it compares to an eventual efficiency increase due to integration of data-driven and goaldirected control need to be assessed by empirical studies. 


\section{Chapter 9}

\section{Conclusions}

In this thesis, we focussed on two issues regarding the blackboard architecture: what, exactly, is the blackboard architecture and what is its applicability. These issues can not be addressed completely in isolation. On the one hand, the nature of the blackboard architecture implies its applicability. Thus, a thorough understanding of the applicability of the blackboard architecture must be preceded by a thorough understanding of the architecture itself. On the other hand, additional understanding of the architecture itself can be obtained by observing applications of the architecture to any number of domains. Consequently, throughout most of this thesis the two focus points of this study were developed together.

However, we still can identify some chapters and sections that concentrated on the nature of the architecture and others that focused on the applicability of the architecture. We use this subdivision to review the work described in this thesis and to present our main conclusions. In the following, Section 9.1 focuses on the nature of the blackboard architecture, while Section 9.2 concentrates on the applicability of the blackboard architecture. More detailed conclusions then presented here can be found in the evaluations of most preceding chapters.

When concerned with applied research in the area of blackboard architectures, an important goal should be the eventual development of a blackboard methodology. Such a methodology should provide guidelines for giving answers to the following four questions: 
1. When is a blackboard architecture appropriate?

2. Which particular blackboard architecture is best suited for a specific application domain?

3. How can models for a specific application domain be mapped onto a selected blackboard architecture?

4. How should one organise control in order to achieve an effective and efficient implementation?

The research reported in this thesis is intended as a step towards the realisation of the goal of establishing a blackboard methodology. In Section 9.3 we discuss future research needed for achieving such a methodology.

\subsection{The nature of the blackboard architecture}

The work which concentrated on identifying the nature of the blackboard architecture is described in Chapters 6 and 7 and Appendix A. We chose to use formalisation as a basis for a fundamental. and analytical study of the blackboard architecture, rather than to rely on experience alone or on empirical studies.

Our formalisation was introduced in Chapter 6. The formalisation prow vided us with a means for carefully defining important dimensions in the design of a blackboard architecture and the variation along each dimension. The definitions that together form our formalisation facilitate comparison of blackboard systems and architectures by providing a unified framework and terminology. Furthermore, development of the formal definitions itself helped us to understand better the nature of the blackboard architecture: specifically, because formalisation helps in attaining completeness and consistency.

The formalisation was used to describe a collection of existing, mostly rather influential blackboard architectures. The descriptions can be found in Appendix A. Our formalisation proved to be useful for describing uniformly many relevant architectural aspects of existing blackboard systems and frameworks. However, as discussed in the evaluation of our formalisation in Section 6.8 , not all interesting aspects could be 
described using our formal definitions. This was partly due to incompleteness and inconsistency in official publications of examined architectures and partly due to insufficient expressive power of our formalisation. These observations suggest several topics for future work, such as extension or modification of our formalisation and generation of descriptions based on actual blackboard architectures instead of imperfect publications. Additionally, one may describe other architectures than the fourteen blackboard architectures described in Appendix A, including 'non-blackboard' architectures, to refine analyses as presented in Chapter 7 .

We studied the descriptions in Appendix A to obtain answers to the four basic questions posed in Chapter 1 . These answers were presented in Chapter 7. In particular, this study led to insights in

- the possible variation within the blackboard architecture (both in existing implementations and in imaginable, but not yet existing, implementations),

- the relation between a specific option and resulting features of the architecture, and

- the characteristics distinguishing the blackboard architecture from other computational frameworks.

The insights regarding this last issue, addressed in Section 7.3, provide an actual and precise description of the nature of the blackboard archi. tecture.

As we were able to obtain answers to our basic questions, our strategy of using formalisation for uniformly describing blackboard architectures proved to be valid for gaining a better understanding of the architecture. We expect that formalisation can be used successfully as well for increasing insights in the nature of other computational frameworlss, such as object orientation.

We conclude this section with two general conclusions derived from the work presented in Chapters 6 and 7 and Appendix A. Firstly, as described in Chapter 7 , we found several opportunities for extending current blackboard architecture technology by making some definitions of aspects within the ardhitecture dynamic. Examples of such aspects are 
blackboard interaction, blackboard definition, knowledge source conditions, knowledge source expertise, and knowledge source composition. Adaptivity and dynamic reconfigurability of the blackboard architecture to account for a changing environment or to incorporate learning from past experience provide interesting (and we expect beneficial) areas for research.

Secondly, we found that publication of reasons for selecting specific options in a blackboard architecture for a particular application is much less common than publication of reasons for selecting the blackboard architecture as such. The development of a blackboard methodology would be supported greatly by discussions of reasons for selecting certain options.

\subsection{Applicability of the blackboard architec- ture}

In Chapter 2, we discussed, among other things, existing applications of the blackboard architecture. Four main categories of applications could be identified: interpretation, monitoring over time, configuration and planning, and control (see Section 2.6). Apart from these application categories the blackboard architecture can be used effectively for developing concurrent systems, as illustrated by several examples in Section 2.4 .

Three examples of specific realisations of the blackboard architecture are described in Chapters 3,4 , and 5 , respectively. In Chapter 3 , we described the blackboard framework Blondie. Blondie was developed in order to gain a better understanding of the blackboard architecture than seemed to be possible from studying existing literature only. Understanding the literature and designing our own blackboard framework took us several times longer than actually implementing Blondie. This illustrates that the blackboard architecture as a computational framework is rather complex, though the basic components constituting a blackboard system or framework are reasonably simple. In order to aid closing the gap between publications on the blackboard architecture and actual descriptions of how to implement a blackboard system or framework, we included a functional description of Blondie in (Lippolt et al., 
1986). This functional description has been used several times by others as a basis for implementation of other blackboard architectures (Aleven, 1988; Giguere et al., 1988; Nalbach \& Stawicki, 1989).

In Chapter 4, we described the parallel blackboard framework BlondieII. This framework was implemented for the application domain of robot control. This work contributed in two areas. Firstly, in Blondie-II we actually implemented parallel execution of knowledge sources. Until then parallel execution of knowledge sources was mostly only noted as an opportunity for introducing concurrency in the blackboard architecture, which seemed quite natural due to the modularity of the architecture and the nature of the blackboard metaphor. Notwithstanding this natural opportunity, it became apparent that parallel execution of knowledge sources is restricted by certain factors such as shared resources. One of these shared resources is the blackboard. Concurrent access of the blackboard may lead to undesirable inconsistency of data on the blackboard. We studied ways for preserving consistency leading to the implementation of mechanisms for handling read locks and write locks for specific regions on the blackboard.

Secondly, during the design of Blondie-II we studied the applicability of the blackboard architecture for robot control. Several characteristics of the blackboard architecture make it suitable for this domain. Of these we mention the ability to incorporate diverse components and the suitability for opportunistic, event-driven control. Although the blackboard architecture may not be sufficient in all kinds of robot control applications (see evaluation in Section 4.4), we intuit, based on our own experience and on results found in the literature (see Section 2.6.4), that the blackboard architecture still provides a useful framework in less demanding robot control applications.

Our third implementation of a blackboard architecture is Blondie-III, described in Chapter 5. Blondie-III is a distributed blackboard framework. Chapter 5 also describes an application of Blondie-III to a networkconfiguration problem. We used Blondie-III and the application to demonstrate three distinct points.

Firstly, both the blackboard (for asynchronous communication) and event-based control (for reacting adequately to newly arriving information) make blackboard systems suitable as 'building blocks' in distributed problem-solving networks. 
Secondly, by successfully realising a blackboard application for a configuration application, we illustrated the feasibility of using the blackboard architecture for such applications. This is further illustrated by the discussion of other blackboard applications in configuration and planning domains in Section 2.6.3.

Thirdly, the use of a distributed blackboard framework in an application domain which is inherently distributed (both geographically and functionally) showed that distributed blackboard frameworks specifically and Distributed Artificial Intelligence systems generally provide useful tools for creating systems dealing with or even exploiting such inherent distribution of the application domain.

The efficiency and effectiveness of the control unit of a blackboard system will usually be decisive for the success of a particular application. In Chapter 8 we argued that in certain applications, in order to obtain sufficient efficiency and effectiveness, integration of goal-directed control with data-driven control, which is more natural in blackboard systems, will be necessary. We also showed that true integration of data-driven and goal-directed control has not been realised in current blackboard architectures. Chapter 8 therefore presents a proposal for a new approach to integration of data-driven and goal-directed control based on a dynamic network. Our approach forms an adaptation of Maes' sprending activation network for action selection (Maes, 1989a; Maes, 1989b). This adaptation involves an extension enabling such an 'activation network' to deal with different instantiations for the same knowledge source. We showed that, except for some restrictions which may be insubstantial in many domains, a network can be defined which combines information about data and goals to select knowledge sources for execution. Future experiments with this approach in real-world applications are necessary to validate this approach in practice.

We would like to conclude this section with some general remarks regarding the applicability of the blackboard architecture. Whilst the blackboard architecture has been conceived and developed mainly within the field of Artificial Intelligence, it is our view that the architecture is much more generally applicable. We saw in Chapters 2, 4, and 5 that there may exist several reasons for choosing (parts of) the blackboard architecture as a framework for a computer program. It is essential that one has an open mind with respect to the architecture and is able to view 
the blackboard architecture not only as a cognitive model with a certain associated 'minimal' configuration, but also as a program architecture providing any of a number of features, according to the specific components included in this program architecture. With these considerations in mind, using (parts of) a blackboard architecture provides facilities for implementing:

- asynchronous, address-less communication between otherwise independent programs,

- representation (on the blackboard) of what can be done, should be done, and must be done eventually,

- integration of systems and programs, which may have diverse internal operations,

- event-driven, data-driven, or opportunistic processing,

- explicit control of activities, and

- modularity.

Of these, modularity provides, in turn, facilities for: a) dividing a problem in manageable and solvable subproblems; b) obtaining flexibility regarding the exchange of components within the system; c) obtaining flexibility of control; and d) achieving efficiency improvements. This last aspect may seem strange, as blackboard architectures are normally not associated with efficiency due to the apparent cost involved with the flexibility of the architecture. However, modularity can in fact greatly increase efficiency as has been illustrated, e.g., in (McCracken, 1979); see also page 48 .

\subsection{Future methodological work}

The blackboard metaphor as such is not a complex concept: it can be explained and understood in simple terms. Nor is implementing a specific instance of the blackboard architecture from a given design a difficult task: we implemented blackboard architectures with reasonably little effort. Yet, the complexity of problem domains and the possible variation 
in design and implementation options for the blackboard architecture make developing a blackboard system a difficult task. A blackboard methodology is needed to assist in this task. The work presented in this thesis should be seen as contributing towards the development of such a methodology. In this section we identify additional, future work leading to a blackboard methodology.

We recognize four different subtasks in the development of a blackboard system. Each subtask constitutes an area for research.

1. When the blackboard architecture is considered as an option for implementing a system for a specific application domain, it must be decided whether the blackboard architecture provides a good, or even optimal, framework for that domain. In order to determine whether the blackboard architecture is appropriate we need clear insights not only regarding the blackboard architecture, but also regarding alternative computational frameworks.

2. When the blackboard architecture is selected for a specific application domain, it becomes necessary to develop a particular blackboard architecture which is best suited for that domain. This is done by choosing among design and implementation options in order to produce an architecture with the desired characteristics for building an application. Knowledge of the relations between options and characteristics is imperative for making such choices.

3. Given a particular blackboard architecture and a specific application domain, the domain model must be mapped onto the architecture. It must be decided, e.g., what constitutes a knowledge source and how the blackboard should be subdivided. This involves a thorough investigation of the domain to find candidates for knowledge sources, expected interactions between those candidates, etc.

4. Even when the three previous tasks have been completed successfully, an effective and efficient control mechanism must be selected and implemented. It has proved to be extremely difficult to combine in a meaningful way a large amount of relevant, but often diverse information to decide which activity should be performed next. 
A general, encompassing theory of blackboard control does not yet exist.

The following lists possible future work in each of these four task areas.

Ad 1. We used formalisation as a basis for determining the nature of the blackboard architecture (see Section 7.3). This is one step towards the development of a methodology for deciding whether a blackboard architecture is appropriate. Formalisation of related computational frameworks (e.g., rule-based programming and objectoriented programming) is not only an interesting task for increasing understanding of those frameworks, it is in our view also necessary to establish, as precisely as possible, the differences and similarities between such frameworks ${ }^{1}$, and to determine the advantages and disadvantages of selecting one framework over another.

Ad 2. Accumulation of a large amount of experience with the blackboard architecture provides one way to acquire knowledge about possible options and their relationship to characteristics. However, we argued that an additional, more fundamental and analytical study is necessary, and we performed a formal study of the blackboard architecture as part of such a fundamental and analytical study.

This formalisation itself is incomplete, as it can be augmented with the formalisation of more details (e.g., by further specifying possible blackboard interactions and flow of control), and with a formalisation of control. Formalisation, leswever, will not be sufficient for developing a complete picture: of whe relationships between options and characteristics. Importint results can be obtained by performing empirical comparisons of diflerent architectural designs and implementations, both in specifically creatcd test environments and in real-world applications.

\footnotetext{
${ }^{1}$ We feel that a clear boundary between different frameworks will not wsually exist. Related frameworks often are distinguished according to gradual differences between options along a number of possibly interrelated dimensions, where transitions from one framework to another are often a matter of opinion. An example of this is provided by the fuzzy distinction between a working memory in rule-based systems and a blackboard in blackboard systems.
} 
Ad 3. Experiments are needed also to increase insight into how to map an application onto a blackboard architecture. Finding a methodology for mapping an application onto a blackboard architecture is much more difficult than finding a methodology for the previous two tasks, since those two tasks are confined to the restricted domain of blackboard architectures, while mapping involves the rather unrestricted domain of all possible applications. We expect that empirical studies will provide general guidelines for mapping applications onto architectures. A more fundamental approach via, e.g., formalisation is currently probably not feasible:

Ad 4. The formalisation of control and the dynamics of blackboard applications as a result of control were left out of our studies, as it forms a separate, domain-dependent problem area. In Section 6.7 we noted several initiatives towards formalisation of control in blackboard systems. We feel that those initiatives constitute important work which will contribute significantly to our understanding of control and the development of a methodology for control in blackboard systems.

The development of a methodology for the blackboard architecture is a task which should be the combined effort of a research community, rather than that of an individual. Validation and verification of the work presented in this thesis or similar work is essential to arrive at a well-accepted methodology. 


\section{Bibliography}

(Adams \& Bisiani, 1986) D.A. Adams and R. Bisiani, The CarnegieMellon University distributed speech recognition system, Speech Technology, pp. 14-23, 1986.

(Addison \& Leon, 1987) E.R. Addison and B.D. Leon, A blackboard architecture for the control of the flow of information in a distributed sensor network, in: Proceedings First Blackbaard Systems Workshop, Seattle, WA, 1987.

(Adey, 1988) S. Adey, High level control of simulated ships and aircraft, in: Proceedings ECAI-88, pp. 442-444, München, 1988.

(Agre, 1990) P.E. Agre, Book review on "Plans and Situated Actions: The Problem of Human-Machine Communications", by L.A. Suchman, Artificial Intelligence, 43:369-384, 1990.

(Aiello, 1983) N. Aiello, A comparative study of control strategies for Expert Systems: AGE implementation of three variations of PUFF, HPP 83-33, Stanford University, Stanford, CA, 1983.

(Aiello, 1986) N. Aiello, User-directed control of parallelism: The CAGE system, KSL 86-31, Stanford University, Stanford, CA, 1986.

(Aleven, 1988) V. Aleven, Blackboard approach to the interpretation of Thallium-201 heart scans (in Dutch), Technical Report, Department of Electrical Engineering, Delft University, 1988.

(Andress \& Kak, 1988) K.M.Andress and A.C. Kak, Evidence accunulation and flow of control in a hierarchical spatial reasoning system, AI Magazine, 1988(2):75-94, 1988. 
(Arlabosse et al., 1988) F. Arlabosse, N.V. Duong, and E. Gaussens, Blackboard and alternative architectural design for two realscale KBS industrial applications, in: Proceedings Second Blackboard Systems Workshop, pp. 173-191, St. Paul, MN, 1988.

(Ayel, 1988) J. Ayel, A conceptual supervision model in computer integrated manufacturing, in: Proceedings ECAI-88, pp. 427-432, München, 1988.

(Bachimont, 1991a) B. Bachimont, DOTMS: a dynamic object-based thruth maintenance system to manage consistency in a blackboard, in: Proceedings 11th International Workshop on Expert Systems and their Applications, General Conference Second Generation Expert Systems, pp. 109-122, Avignon, 1991.

(Bachimont, 1991b) B. Bachimont, A logical framework to manage coherence and convergence in blackboard architectures: a proposal, in: Proceedings Fifth Blackboard Systems Workshop, Anaheim, CA, 1991.

(Baeten \& Weijland, 1990) J.C.M. Baeten and W. P. Weijland, Process Algebra, volume 18 of Cambridge Tracts in Theoretical Computer Science, Cambridge University Press, Cambridge, MA, 1990.

(Bakker, 1986) J.J. Bakker, An architecture for the control of a flexible manufacturing system (in Dutch), in: Perspectieven in de Robotica, Delft, 1986.

(Balzer et al., 1980) R. Balzer, L.D. Erman, P. London, and C. Williams, Hearsay-III: A domain-independent framework for Ex. pert Systems, in: Proceedings AAAl-80, pp. 108-110, Stanford, CA, 1980.

(Barrett et al., 1985) R. Barrett, A. Ramsay, and A. Sloman, POP-11: a Practical Language for Artificial Intelligence, Ellis Horwood Ltd., Chichester, UK, 1985.

(Baum et al., 1989) L.S. Baum, R.T. Dodhiawala, and V. Jagannathan, The Erasmus system, in: V. Jagannathan, R. Dodhiawala, 
and L.S. Baum, eds., Blackboard Architectures and Applications, pp. 347-370, San Diego, CA, 1989. Academic Press.

(Bisiani, 1986) R. Bisiani, A software and hardware environment for developing AI applications on parallel processors, in: Proceedings AAAI-86, pp. 742-747, Philadelphia, PA, 1986.

(Bisiani \& Forin, 1988) R. Bisiani and A. Forin, Multilanguage parallel programming of heterogeneous machines, IEEE Transactions on Computers, C-37(8):930-945, 1988.

(Bisiani et al., 1987) R. Bisiani, F. Alleva, A. Forin, R. Lerner, and M. Bauer, The architecture of the AGORA environment, in: M.N. Huhns, ed., Distributed Artificial Intelligence, Research Notes in Artificial Intelligence, chapter 4, pp. 99-117. Pitman, London, 1987.

(Blevins et al., 1989) D.M. Blevins, B.S. Miller, and L.S. Baum, Benchmarking blackboards to support cockpit information management, in: Proceedings Third Blackboard Systems Workshop, pp. 59-66, Detroit, MI, 1989.

(Bond \& Gasser, 1988) A.H. Bond and L. Gasser, eds., Readings in Distributed Artificial Intelligence, Morgan Kaufmann Publishers, Inc., San Mateo, CA, 1988.

(Boureau \& Hayes-Roth, 1989) L. Boureau and B. Hayes-Roth, Distributed perception and reasoning knowledge in blackboard architectures, in: Proceedings Third Blackboard Systems Workshop, pp. 67-75, Detroit, MI, 1989.

(Brajnik et al., 1988) G. Brajnik, G. Guida, M. Pighin, and C. Tasso, ADL: a language and tool for designing blackboard system architectures, in: Proceedings Second Blackboard Systems Workshop, pp. 1-18, St. Paul, MN, 1988.

(Braver et al., 1987) W. Brauer, W. Reisig, and G. Rozenberg, eds., Petri Nets: Central Models and Their Properties - Advances in Petri Nets 1986, Part I, Springer Verlag, Berlin, 1987.

(Brogi et al., 1988) A. Brogi, R. Filippi, M. Gaspari, and F. Turini, An expert system for data fusion based on a blackboard architecture, in: Proceedings Bih International Workshop on Expert 
Systems and their Applications, Specialized conferences, pp. 147-165, Avignon, 1988.

(Brunessaux \& Chevrier, 1990) S. Brunessaux and V. Chevrier, Facilitating the design of a blackboard-based application through explanation, in: Proceedings Fourth Blackboard Systems Workshop, Boston, MA, 1990.

(Buteau, 1989) B.L. Buteau, A generic framework for the implementation of distributed blackboard systems, in: Proceedings Third Blackboard Systems Workshop, pp. 147-155, Detroit, MI, 1989.

(Chandraselkaran \& Mittal, 1983) B. Chandrasekaran and S. Mittal, Deep versus compiled knowledge approaches to diagnostic problem-solving, International Journal of Man-Machine Studies, 19:425-436, 1983.

(Ciscon \& Johnson, 1990) L. Ciscon and D.H. Johnson, Distributed blackboard for robot path planning in a heterogeneous computer environment, in: Proceedings Fourth Blackboard Systems Workshop, Boston, MA, 1990.

(Cohen et al., 1989) R.M. Cohen, T.P. McCandless, and E. Rich, A problem solving approach to human-computer interface management, in: Proceedings Third Blachboard Systems Workshop, pp. 213-227, Detroit, MI, 1989.

(Corkill, 1988) D.D. Corkill, Design alternatives for parailel and distributed blackboard systems, in: Proceedings Second Blachboard Systems Workshop, pp. 89-106, St. Paul, MN, 1988.

(Corkill \& Lesser, 1983) D.D. Corkill and V.R. Lesser, The use of metalevel control for coordination in a distributed problem solving network, in: Proceedings IJCAT-89, pp. 748-756. Karlsruhe, 1983.

(Corkill et al., 1982) D.D. Corkill, V.R. Lesser, and E. Hudlicka, Unifying data-directed and goal-directed control: an example and experiments, in: Proceelings AAAI-8\%, pp. 143-14T, Pittsburgh, PA, 1982. 
(Corkill et al., 1986) D.D. Corkill, K.Q. Gallagher, and K.E. Murray, GBB: a generic blackboard development system, in: Proceedings AAAI-86, pp. 1008-1014, Philadelphia, PA, 1986.

(Corkill et al., 1987) D.D. Corkill, K.Q. Gallagher, and P.M. Johnson, Achieving flexibility, efficiency, and generality in blackboard architectures, in: Proceedings AAAI-87, pp. 18-23, Seattle, WA, 1987.

(Craig, 1986) I.D. Craig, The Ariadne-1 blackboard system, The Computer Journal, 29(3):235-240, 1986.

(Craig, 1987a) I.D. Craig, The BB-SR System, Technical Report No. 94, University of Warwick, 1987.

(Craig, 1987b) I.D. Craig, A distributed blackboard architecture, in: Proceedings First Blackboard Systems Workshop, Seattle, WA, 1987.

(Craig, 1989) I.D. Craig, The CASSANDRA Architecture: Distributed Control in a Blackboard System, Ellis Horwood Ltd., Chichester, UK, 1989.

(Craig, 1991) I.D. Craig, Formal Specification of Advanced AI Architectures, Ellis Horwood Ltd., Chichester, UK, 1991.

(Cullingford, 1981) R.E. Cullingford, Integrating knowledge sources for computer "understanding" tasks, IEEE Transactions on Systems, Man, and Cybernetics, SMC-11(1):52-60, 1981.

(Dai et al., 1990) H. Dai, T.J. Anderson, and F.C. Monds, A framework for real-time problem solving, in: Proceedings ECA1-90, pp. 179-185, Stockholm, 1990.

(Dai et al., 1991) H. Dai, T.J. Anderson, and F.C. Monds, On the management of real-time information in a parallel expert system, in: Proceedings 11th International Workshop on Expert Systems and their Applications, General Conference Tools, Techniques, and Methods, pp. 455-467, Avignon, 1991.

(Dassow, 1991) J. Dassow, Cooperating/distributed grammar systems with hypothesis languages, Journal of Experimental and Theoretical Artificial Intelligence, 3:11-16, 1991. 
(Davis, 1980) R. Davis, Report on the workshop on distributed AI (1980), SIGART Newsletter, (73):42-52, 1980.

(Davis, 1982) R. Davis, Report on the second workshop on distributed AI (1981), SIGART Newsletter, (80):13-23, 1982.

(Davis \& Hamscher, 1988) R. Davis and W. Hamscher, Model-based reasoning: troubleshooting, in: H.E. Shrobe, ed., Exploring Artificial Intelligence, chapter 8, pp. 297-346, Morgan Kaufmann, Inc., San Mateo, CA, 1988.

(de Kleer, 1986) J. de Kleer, An assumption-based TMS, Artificial Intelligence, $28: 127-162,1986$.

(Decker, 1987) K.S. Decker, Distributed problem-solving techniques: a survey, IEEE Transactions on Systems, Man, and Cybernetics, SMC-17(5):729-740, 1987.

(Dodhiawala et al., 1987) R.T. Dodhiawalla, V. Jagannathan, and L.S. Baum, Erasmus system design: performance issues, in: Proceedings First Blackboard Systems Workshop, Seattle, WA, 1987.

(Doyle, 1979) J. Doyle, A truth maintenance system, Artificial Intelligence, $12: 231-272,1979$.

(Draper et al., 1988) B.A. Draper, R.T. Collins, J. Brolio, A.R. Hanson, and E.M. Riseman, Issues in the development of a blackboardbased schema system for image understanding, in: R.S. Engelmore and A.J. Morgan, eds., Blackboard Systems, pp. 189218. Addison-Wesley, Reading, MA, 1988.

(Dreschler-Fischer, 1986) L.S. Dreschler-Fischer, A blackboard system for dynamic stereo matching, in: Proceedings Intelligent $\mathrm{Au}$ tonomous Systems, pp. 189-202, Amsterdam, 1986.

(Durfee, 1988) E.H. Durfee, Coordination of Distributed Problem Solvers, Kluwer Academic Publishers, Boston, MA, 1988.

(Durfee \& Lesser, 1987) E.H. Durfee and V.R. Lesser, Using partial global plans to coordinate distributed problem solvers, in: Proceedings IJCA I-87, pp. 875-883, Milan, 1987. 
(Durfee et al., 1985) E.H. Durfee, V.R. Lesser, and D.D. Corkill, Increasing coherence in a distributed problem solving network, in: Proceedings IJCAI-85, pp. 1025-1030, Los Angeles, CA, 1985.

(Durfee et al., 1987) E.H. Durfee, V.R. Lesser, and D.D. Corkill, Coherent cooperation among communicating problem solvers, IEEE Transactions on Computers, C-36:1275-1291, 1987.

(Engelmore \& Morgan, 1988) R.S. Engelmore and A.J. Morgan, eds., Blackboard Systems, Addison-Wesley, Reading, MA, 1988.

(Engelmore \& Terry, 1979) R.S. Engelmore and A. Terry, Structure and function of the Crysalis system, in: Proceedings IJCAI-79, pp. 250-256, Tokyo, 1979.

(Ensor \& Gabbe, 1985) J.R. Ensor and J.D. Gabbe, Transactional blackboards, in: Proceedings IJCAI-85, pp. 340-344, Los Angeles, CA, 1985.

(Erman \& Lesser, 1978) L.D. Erman and V.R. Lesser, System engineering techniques for artificial intelligence systems, in: A. Hansen and E. Riseman, eds., Computer Vision Systems, pp. 37-45. Academic Press, New York, NY, 1978.

(Erman et al., 1980) L.D. Erman, F. Hayes-Roth, V.R. Lesser, and D.R. Reddy, The Hearsay-II speech-understanding system: intem grating knowledge to resolve uncertainty, ACM Computing Surveys, 12(2):213-253, 1980.

(Erman et al., 1981) L.D. Erman, P.E. London, and S.F. Fickas, The design and an example use of Hearsay-III, in: Proceedings IJCA I-81, pp. 409-415, Vancouver, BC, 1981.

(Fehling \& Erman, 1983) M. Fehling and L. Erman, Report on the third annual workshop on distributed Artificial Intelligence, SIGART Newsietter, (84):3-12, 1983.

(Fehling et al., 1987) M.R. Fehling, S. Forrest, and B.M. Wilber, The Heuristic Control Virtual Machine, in: Proceedings Blackboard Systems Workshop, Seattle, WA, 1987. 
(Fehling et al., 1989) M.R. Fehling, A.M. Altman, and B.M. Wilber, The Heuristic Control Virtual Machine: an implementation of the Schemer computational model of reflective, real-time problem-solving, in: V. Jagannathan, R. Dodhiawala, and L.S. Baum, eds., Blackboard Architectures and Applications, pp. 191-218. Academic Press, San Diego, CA, 1989.

(Fennell \& Lesser, 1977) R.D. Fennell and V.R. Lesser, Parallelism in artificial intelligence problem solving: A case study of Hearsay-II, IEEE Transactions on Computers, C-26(1):98$111,1977$.

(Fernandez de Castro et al., 1988) M.I. Fernandez de Castro, A. Diaz de Llarraza Sanchez, and M.F. Verdejo Maillo, A cooperative architecture for tutoring tasks, in: Proceedings 8th International Workshop on Expert Systems and their Applications, Volume 2, pp. 107-126, Avignon, 1988.

(Fikes, 1987) R. Fikes, Integrating blackboards into the KEE objectoriented modeling environment, in: Proceedings First Blackboard Systems Workshop, Seattle, WA, 1987.

(Fikes \& Nilsson, 1971) R.E. Fikes and N.J. Nilsson, STRIPS: a new approach to the application of theorem proving to problem solving., Artificial Intelligence, 2(3/4):189-208, 1971.

(Findler \& Gao, 1987) N.V. Findler and J. Gao, Dynamic hierarchical control for distributed problem solving, Data 8 Knowledge Engineering, 2:285-301, 1987.

(Fleisch, 1990) B.D. Fleisch, Using distributed shared memory as a framework for a distributed blackboard, in: Proceedings Fourth Blackboard Systems Workshop, Boston, MA, 1990.

(Florescu et al., 1988a) A. Florescu, E.P.M. van Liempd, and H. Velthuijsen, BLONDIE-III: an environment for distributed problem solving, Internal Report 88 DNL/16, PTT Research, Leidschendam, 1988.

(Florescu et al., 1988b) A. Florescu, E.P.M. van Liempd, and H. Velthuijsen, A distributed blackboard architecture for network 
configuration (in Dutch), in: Proceedings AI Toepassingen '88, pp. 637-644, The Hague, 1988.

(Florescu et al., 1988c) A. Florescu, E.P.M. van Liempd, and H. Velthuijsen, A distributed implementation of a network configuration problem, Memorandum 1844 DNL/88, PTT Research, Leidschendam, 1988.

(Gallagher et al., 1988) K.Q. Gallagher, D.D. Corkill, and P.M. Johnson, GBB Reference Manual, GBB version 1.2, COINS Technical Report 88-66, Computer and Information Science, University of Massachusetts, Amherst, MA, 1988.

(Gallestigni et al., 1989) I. Gallestigni, M. Bhandaru, H. Nawab, V.R. Lesser, and D.D. Corkill, Implementing a blackboard-based sound classification system: a case study, in: Proceedings Third Blackboard Systems Workshop, pp. 247-253, Detroit, MI, 1989 .

(Gasser, 1987) L. Gasser, The 1985 workshop on distributed Artificial Intelligence, AI Magazine, 1987(2):91-97, 1987.

(Gasser \& Huhns, 1989) L. Gasser and M.N. Huhns, eds., Distriounted Artificial Intelligence, volume II, Research Notes in Artificial Intelligence. Pitman, London, 1989.

(Giguere et al, 1988) C. Gignere, T. Lim, and R. McGill, A blackboard expert system shell, ELE 1635 Project Report, University of Toronto, Department of Electrical Engineering, Toronto, 1988.

(Gong \& Haton, 1989) Y. Gong and J.P. Haton, The specialist society - a mixed framework for multi-agent reasoning, in: Proceedings Third Blackboard Systems Workshop, pp. 33-46, Detroit, MI, 1989.

(Grumbach \& Ducasse, 1983) A. Grumbach and M. Ducasse, An Expert System shell for signal interpretation. The hypothesis management module, in: Proceedings Expert Systems '83, pp. 53-62, 1.983. 
(Harmon, 1989) S.Y. Harmon, A decentralized distributed blackboard system, in: Proceedings Third Blackboard Systems Workshop, pp. 157-172, Detroit, MI, 1989.

(Haton et al., 1987) J.P. Haton, B. Maitre, H. Laasri, and T. Mondot, ATOME, in: Proceedings First Blackboard Systems Workshop, Seattle, WA, 1987.

(Hausen-Tropper, 1989) E. Hausen-Tropper, A blackboard architecture for weather preconditions, in: Proceedings Third Blackboard Systems Workshop, pp. 191-202, Detroit, MI, 1989.

(Hayes-Roth, 1984) B. Hayes-Roth, BB1: an architecture for blackboard systems that control, explain, and learn about their own behavior, STAN-CS-84-1034, Stanford University, Stanford, CA, 1984.

(Hayes-Roth, 1985) B. Hayes-Roth, A blackboard architecture for control, Artificial Intelligence, 26:251-321, 1985.

(Hayes-Roth \& Hayes-Roth, 1979) B. Hayes-Roth and F. Hayes-Roth, A cognitive model of planning, Cognitive Science, 3:275-310, 1979.

(Hayes-Roth \& Hewett, 1985) B. Hayes-Roth and M. Hewett, Learning control heuristics in BB1, HPP 85-2, Stanford University, Stanford, CA, 1985.

(Hayes-Roth \& Lesser, 1977) F. Hayes-Roth and V.R. Lesser, Focus of attention in a distributed-logic speech understanding system, in: Proceedings IJCAI-77, pp. 416-420, 1977.

(Hayes-Roth et al., 1983) F. Hayes-Roth, D.A. Waterman, and D.B. Lenat, eds., Building Expert Systems, Addison-Wesley, Reading, MA, 1983.

(Hayes-Roth et al., 1986a) B. Hayes-Roth, B. Buchanan, O. Lightarge, M. Hewett, R. Altman, J. Brinkley, C. Cornelius, B. Duncan, and $O$. Jardetzky, PROTEAN: deriving protein structure from constraints, in: Proceedings AAAI-86, pp. 904-909, Philadelphia, PA, 1986. 
(Hayes-Roth et al., 1986b) B. Hayes-Roth, A. Garvey, M.V. Johnson, and $M$. Hewett, A layered environment for reasoning about action, KSL 86-38, Stanford University, Stanford, CA, 1986.

(Hendler et al., 1990) J. Hendler, A. Tate, and M. Drummond, AI planning: systems and techniques, AI Magazine, 11(2):61-77, 1990.

(Herik \& Henseler, 1986) H.J. van den Herik and J. Henseler, Control mechanisms in the parallel knowledge-based system HYDRA, in: Proceedings Artificial Intelligence and Advanced Computer Technology Conference/Exhibition, pp. 1-12, Wiesbaden, 1986. TCM Expositions, Ltd., Hants, UK.

(Herik et al., 1988) H.J. van den Herik, G. Honderd, W. Jongkind, E.J.H. Kerckhoffs, and J.D. Verwey, Rule-based control of a mobile robot vehicle: a simulation study, in: Proceedings of the User Working Conference for Users of Simulation Hardware, Software, and Intelliware, pp. 264-269, Ostende, 1988.

(Hoare, 1985) C.A.R. Hoare, Communicating Sequential Processes, Prentice-Hall International Series in Computer Science. Prentice-Hall, London, 1985.

(Hudlicka \& Lesser, 1984) E. Hudlicka and V.R Lesser, Meta-level control through fault detection and diagnosis, in: Proceedings AAAI-84, pp. 153-161, Austin, TX, 1984.

(Hudlicka \& Lesser, 1987) E. Hudlicka and V.R Lesser, Modeling and diagnosing problem-solving system behavior, IEEE Transactions on Systems, Man, and Cybernetics, SMC-17(3):407-419, 1987.

(Huhns, 1987) M.N. Huhns, Distributed Artificial Intelligence, Research Notes in Artificial Intelligence. Pitman, London, 1987.

(Jagannathan, 1989) V. Jagannathan, Realizing the concurrent blackboard model, in: V. Jagannathan, R. Dodhiawala, and L.S. Baum, eds., Blackboard Architectures and Applications, pp. 85-97, San Diego, CA, 1989. Academic Press. 
(Jagannathan et al., 1987) V.Jagannathan, L.S. Baum, and R.T. Dodhiawala, Designing a distributed blackboard system with Erasmus, in: Proceedings First Blackboard Systems Workshop, Seattle, WA, 1987.

(Jagannathan et al., 1989) V. Jagannathan, R. Dodhiawala, and L.S. Baum, eds., Blackboard Architectures and Applications, Academic Press, San Diego, CA, 1989.

(Johnson \& Hayes-Roth, 1986) M.V. Johnson and B. Hayes-Roth, Integrating diverse reasoning methods in the BB1 blackboard control architecture, KSL 86-76, Stanford University, Stanford, CA, 1986.

(Jones et al., 1986) J. Jones, M. Millington, and P. Ross, A blackboard shell in Prolog, in: Proceedings ECAI-86, pp. 428-436, Brighton, 1986.

(Jones et al, 1990) P.M. Jones, C.M. Mitchell, and K.S. Rubin, Validation of intent inferencing by a model-based operator's associate, International Journal of Man-Machine Studies, 33:177202,1990 .

(Kaiser et al., 1988) K. Kaiser, L. Baum, D. Blevins, B. Miller, and V. Jagannathan, Adapting the blackboard model for cockpit information management, in: Proceedings Second Blackboard Systems Workshop, pp. 193-209, St. Paul, MN, 1988.

(Kak et al., 1986) A.C. Kak, K.L. Boyer, C.H. Chen, R.J. Safranek, and H.S. Yang, A knowledge-based robotic assembly cell, IEEE Expert, pp, 63-83, 1986 .

(Kelemen, 1991) J. Kelemen, Syntactical models of distributed cooperative systems, Journal of Experimental and Theoretical Artificial Intelligence, 3:1-10, 1991.

(Laasri el al, 1988a) H. Laasri, B. Maitre, and J.P. Haton, Hybrid control to achieve flexibility and efficiency in blackboard-based systems, CRIN-88-R-033, Centre de recherche en informatique de Nancy, Nancy, 1988. 
(Laasri et al., 1988b) H. Laasri, B. Maitre, T. Mondot, F. Charpillet, and J.P. Haton, ATOME: A blackboard architecture with temporal and hypothetical reasoning, CRIN-88-R-013, Centre de recherche en informatique de Nancy, Nancy, 1988.

(Leao \& Talukdar, 1988) L.V. Leao and S.N. Talukdar, COPS: a system for constructing multiple blackboards, in: A.H. Bond and L. Gasser, eds., Readings in Distributed Artificial Intelligence, chapter 7.3, pp. 547-556. Morgan Kaufmann Publishers, Inc., San Mateo, CA, 1988.

(Lehnert, 1989) K. Lehnert, A blackboard approach to planning and execution in decision systems, in: Proceedings Third Blackboard Systems Workshop, pp. 203-212, Detroit, MI, 1989.

(Lenat et al., 1983) D.B. Lenat, A. Clarkson, and G. Kiremidjian, An expert system for indications \& warning analysis, in: Proceedings IJCAI-83, pp. 259-262, Karlsruhe, 1983.

(Lesser \& Corkill, 1981) V.R. Lesser and D.D. Corkill, Functionally accurate, cooperative distributed systems, IEEE Transactions on Systems, Man, and Cybernetics, SMC-11(1):81-96, 1981.

(Lesser \& Corkill, 1983) V.R. Lesser and D.D. Corkill, The distributed vehicle monitoring testbed, AI Magazine, 4(3):15-33, 1983.

(Lesser \& Erman, 1988) V.R. Lesser and L.D. Erman, A retrospective view of the Hearsay-II architecture, in: R.S. Engelmore and A.J. Morgan, eds., Blackboard Systems, pp. 87-121. AddisonWesley, Reading, MA, 1988.

(Lesser et al, 1975) V.R. Lesser, R.D. Fennell, L.D. Erman, and D.R. Reddy, Organization of the Hearsay-II speech understanding system, IEEE Transactions on Acoustics, Speech, and Signal Processing, ASSP-23(1):11--24, 1975.

(Lesser et al., 1988) V.R. Lesser, D.D. Corkill, J.A. Hernandez, and R.C. Whitehair, Goal relationships in blackboard architectures, in: Proceedings Second Blackboard Systems Workshop, pp. 73-87, St. Paul, MN, 1988. 
(Lesser et al., 1989a) V.R. Lesser, D.D. Corkill, R.C. Whitehair, and J.A. Hernandez, Focus of control through goal relationships, in: Proceedings IJCAI-89, pp. 497-503, Detroit, MI, 1989.

(Lesser et al., 1989b) V.R. Lesser, R.C. Whitehair, D.D. Corkill, and J.A. Hernandez, Goal relationships and their use in a blackboard architecture, in: V. Jagannathan, R. Dodhiawala, and L.S. Baum, eds., Blackboard Architectures and applications, chapter 1, pp. 9-26. Academic Press, Inc., 1989.

(Levy \& Finin, 1987) J. Levy and T. Finin, BBC: a blackboard generating system, in: Proceedings First Blackboard Systems Workshop, Seattle, WA, 1987.

(Liempd et al., 1989) E.P.M. van Liempd, A. Florescu, and H. Velthuijsen, A distributed blackboard implementation of a network configuration problem, in: Proceedings Third Blackboard Systems Workshop, pp. 229-245, Detroit, MI, 1989.

(Liempd et al., 1990) E.P.M. van Liempd, H. Velthuijsen, and A. Florescu, BLONDIE-III: a distributed implementation of a network configuration problem, IEEE Expert, 5(4):48-55, 1990.

(Ling et al., 1987) C. Ling, R.T. Dodhiawala, D. Nguyen, and T. Skillman, A parallel VLSI architecture for blackboard systems, in: Proceedings First Blackboard Systems Workshop, Seattle, WA, 1987.

(Lippolt et al., 1986) B.J. Lippolt, H. Velthuijsen, and J.C. Vonk, BLONDIE: a blackboard shell, 1404 DNL/86, PTT Research, Leidschendam, 1986.

(Lippolt et al., 1987) B.J. Lippolt, H. Velthuijsen, and J.C. Vonk, A blackboard shell with multiple blackboards, in: Proceedings KBS-87, pp. 71-78, London, 1987.

(Lister, 1975) A.M. Lister, Fundamentals of Operating Systems, The Macmillan Press Ltd, London, 1975.

(Lumley, 1983) J. Lumley, PROBE, a Prolog blackboard environment, in: Proceedings Expert Systems '83, pp. 126-134, 1983. 
(Lutze, 1988) R. Lutze, Customizing cooperative office procedures by planning, in: Proceedings Conference on Office Automation Systems (ACM Sigois), pp.63-77, Palo Alto, CA, 1988.

(Maes, 1989a) P. Maes, The dynamics of action selection, in: Proceedings IJCAL-89, pp. 991-997, Detroit, MI, 1989.

(Maes, 1989b) P. Maes, A spreading activation network for action selection, in: Proceedings Intelligent Autonomous Systems 2, pp. 875-885, Amsterdam, 1989.

(Märtin \& Waldhör, 1988) C. Märtin and K. Waldhör, BASAR - a blackboard based software architecture, in: Proceedings ECAI-88, pp. 2-4, München, 1988.

(Mathe, 1990) N. Mathe, A blackboard approach to intelligent assistance for space telemanipulation, in: Proceedings Fourth Blackboard Systems Workshop, Boston, MA, 1990.

(McClelland \& Rumelhart, 1981) J.L. McClelland and D.E. Rumelhart, An interactive activation model of context effects in letter perception: Part 1, an account of basic findings, Psychological Review, 88:375-407, 1981.

(McClelland, 1986) J.L. McClelland, The programmable blackboard model of reading, in: J.L. McClelland and D.E. Rumelhart, eds., Parallel Distributed Processing, Vol. 2: Psychological and Biological Models, pp. 122-169. MIT Press, Cambridge, MA, 1986.

(McCracken, 1979) D.L. McCracken, Representation and efficiency in a production system for speech understanding, in: Proceedings IJCAI-79, pp. 556-561, Tokyo, 1979.

(Milne \& Eldridge, 1988) R. Milne and R. Eldridge, Reasoning about relationships, in: Proceedings 8th International Workshop on Expert Systems and their Applications, Specialized con. ferences, pp. 467-478, Avignon, 1988.

(Milner, 1989) R. Milner, Communication and Concurrency, PrenticeHall International Series in Computer Science. Prentice-Hall, London, 1989. 
(Mulder \& Braspenning, 1988) F.W. Mulder and P.J. Braspenning, A complex blackboard system for interpretation of series of images (in Dutch), in: Proceedings AI Toepassingen '88, pp. 627-635, The Hague, 1988.

(Nagao et al., 1988) M. Nagao, T. Matsuyama, and H. Mori, Structural analysis of complex aerial photographs, in: R.S. Engelmore and A.J. Morgan, eds., Blackboard Systems, chapter 9, pp. 219-230. Addison-Wesley, Reading, MA, 1988.

(Nalbach \& Stawicki, 1989) M. Nalbach and J. Stawicki, BB-POL - a blackboard expert system shell for industrial applications, in: Proceedings 5th International Conference on Artificial Intelligence and Information-Control Systems of Robots, Strbske Pleso, 1989.

(Newell, 1962) A. Newell, Some problems of the basic organization in problem-solving programs, in: M.C. Yovits, G.T. Jacobi, and G.D. Goldstein, eds., Proceedings Second Conference on SelfOrganizing Systems, pp. 393-423. Spartan Books, 1962.

(Nii, 1985) H.P. Nii, Research on blackboard architectures at the Heuristic Programming Project, KSI. 85-24, Stanford University, Stanford, CA, 1985.

(Nii, 1986a) H.P. Nii, Blackboard systems: blackboard application systems, blackboard systems from a knowledge engineering perspective, AI Magazine, T(3):82-106, 1986.

(Nii, 1986b) H.P. Nii, Blackboard systems: the blackboard model of problem solving and the evolution of blackboard architectures, AI Magazine, $7(2): 38-53,1986$.

(Nii, 1986c) H.P. Nii, CAGE and POLIGON: Two frameworks for blackboard based concurrent problem solving, KSL 86-41, Stanford University, Stanford, CA, 1986.

(Nii \& Aiello, 1979) H.P. Nii and N. Aiello, AGE (Attempt to Generalize): a knowledge-based program for building knowledgebased programs, in: Proceedings IJCAI-79, pp. 645-655, Tokyo, 1979. 
(Nii \& Feigenbaum, 1978) H.P. Nii and E.A. Feigenbaum, Rule-based understanding of signals, in: D.A. Waterman and F. HayesRoth, eds., Pattern Directed Inference Systems, pp. 483-501. Academic Press, New York, NY, 1978.

(Nii et al., 1988) H.P. Nii, E.A. Feigenbaum, J.J. Anton, and A.J. Rockmore, Signal-to symbol transformation: HASP/SIAP case study, in: R.S. Engelmore and A.J. Morgan, eds., Blackboard Systems, chapter 6, pp. 135-157. Addison-Wesley, Reading, MA, 1988.

(Niyogi \& Srihari, 1987) D. Niyogi and S.N. Srihari, Using a blackboard architecture for control in a knowledge based document understanding system, in: Proceedings SPIE Vol.786 Applications of Artificial Intelligence $V$, pp. 319-326, 1987.

(Pang, 1989) G.K.H. Pang, A blackboard system for the off-line programming of robots, Journal of Intelligent and Robotic Systems, 2:425-444, 1989.

(Pang \& Shen, 1990) G.K.H. Pang and H.C. Shen, Intelligent control of an autonomous mobile robot in a hazardous matrial spill accident - a blackboard structure approach, Robotics and Autonomous Systems, 6:351-365, 1990.

(Pearson, 1988) G. Pearson, Mission planning within the framework of the blackboard model, in: R.S. Engelmore and A.J. Morgan, eds., Blackboard Systems, chapter 21, pp. 433-442. AddisonWesley, Reading, MA, 1988.

(Penders, 1989) J.S.J.H. Penders, Error recovery in a robot system, in: T. Kanade, F.C.A. Groen, and L.O. Hertzberger, eds., Proceedings Intelligent Autonomous Systems 2, pp. 929-936, Amsterdam, 1989. IOS Press.

(Perlis, 1987) D. Perlis, Circumscription, in: S.C. Shapiro, ed., Encyclopedia of Artificial Intelligence, volume 1, pp. 100-103, John Wiley \& Sons, New York, NY, 1987.

(Pomeroy \& Jrving, 1990) B.D. Pomeroy and R. Irving, A blackboard approach for diagnosis in Pilot's Associate, IEEE Expert, $5(4): 39-46,1990$. 
(Pomeroy \& Stein, 1990) B.D. Pomeroy and B.A. Stein, Real-time blackboard scheduling for advanced crewstations, in: Proceedings Fourth Blackboard Systems Workshop, Boston, MA, 1990.

(Potter \& Lawrence, 1987) M.A. Potter and J.L. Lawrence, Tabula: a blackboard environment for knowledge engineering, in: Proceedings First Blackboard Systems Workshop, Seattle, WA, 1987.

(Raulefs, 1987) P. Raulefs, Towards a blackboarả architecture for realtime control of dynamic systems, in: Proceedings First Blackboard Systems Workshop, Seattle, WA, 1987.

(Reddy et al., 1973) D.R. Reddy, L.D. Erman, R.D. Fennell, and R.B. Neely, The Hearsay speech understanding system: an example of the recognition process, in: Proceedings IJCAI-73, pp. 185193, Stanford, CA, 1973.

(Rice, 1986) J.P. Rice, POLIGON, a system for parallel problem solving, KSL 86-19, Stanford University, Stanford, CA, 1986.

(Rice, 1989a) J.P. Rice, The Advanced Architectures Project, AI Magazine, pp. 26-39, 1989.

(Rice, 1989b) J.P. Rice, The design and implementation of Poligon, a. high performance, concurrent blackboard system shell, KSL 89-37, Knowledge Systems Laboratory, Stanford University, Stanford, CA, 1989.

(Rice, 1989c) J.P. Rice, The ELINT application on Poligon: the architecture and performance of a concurrent blackboard system, in: Proceedings IJCAI-89, pp. 212-217, Detroit, MI, 1989.

(Rice \& Aiello, 1989) J.P. Rice and N. Aiello, See how they run ..., the architecture and performance of two concurrent blackboard systems, in: V. Jagannathan, R. Dodhiawala, and L.S. Baum, eds., Blackboard Architectures and Applications, chapter 8, pp. 153-178. Academic Press, Inc., 1989. 
(Roth et al., 1987) S.P. Roth, S.D. Tynor, and J.F. Gilmore, GEST: development of a Blackboard Expert System tool, in: Proceedings First Blackboard Systems Workshop, Seattle, WA, 1987.

(Rubin et al., 1988) K.S. Rubin, P.M. Jones, and C.M. Mitchell, OFMSPERT: inference of operator intentions in supervisory control using a blackboard architecture, IEEE Transactions on Systems, Man, and Cybernetics, SMC-18(4):618-637, 1988.

(Rumelhart \& McClelland, 1982) D.E. Rumelhart and J.L. McClelland, An interactive activation model of context effects in letter perception: Part 2, the enhancement effect and some tests and extensions to the model, Psychological Review, 89:60-94, 1982.

(Rumelhart \& McClelland, 1986) D.E. Rumelhart and J.L. McClelland, eds., Parallel Distributed Processing, Vol. 1: Foundations, MIT Press, Cambridge, MA, 1986.

(Sacerdoti, 1974) E.D. Sacerdoti, Planning in a hierarchy of abstraction spaces, Artificial Intelligence, 5:115-135, 1974.

(Saxena et al., 1990) M.K. Saxena, K.K. Biswas, and P.C.P. Bhatt, DISPROS: a distributed blackboard architecture, in: Proceedings IEA/AIE-90, Charleston, SC, 1990.

(Shafer \& Stentz, 1987) S.A. Shafer and A. Stentz, A communications database for mobile robot navigation, in: Proceedings First Blackboard Systems Workshop, Seattle, WA, 1987.

(Shortliffe \& Buchanan, 1984) E.H. Shortliffe and B.G. Buchanan, A model of inexact reasoning in medicine, in: B.G. Buchanan and E.H. Shortliffe, eds., Rule-Based Expert Systerns, chapter 11, pp. 233-262, Addison-Wesley, Reading, MA, 1984.

(Silverman et al., 1989) B.G. Silverman, J.S. Chang, and K. Feggos, Blackboard System Generator (BSG): an alternative distributed problem-solving paradigm, IEEE Transactions on Systems, Man, and Cybernetics, SMC-19(2):334-355, 1989. 
(Smith, 1980) R.G. Smith, The contract net protocol: high-level communication and control in a distributed problem solver, IEEE Transactions on Computers, C-29(12):1104-1113, 1980.

(Smith, 1985) R.G. Smith, Report on the 1984 distributed Artificial Intelligence workshop, AI Magazine, 6(3):234-243, 1985.

(Sombé, 1990) Group Léa Sombé, Reasoning under incomplete information in Artificial Intelligence: a comparison of formalisms using a single example, International Journal of Intelligent Systems, 5:324-472, 1990.

(Spivey, 1988) J.M. Spivey, Understanding $Z$, Cambridge University Press, Cambridge, UK, 1988.

(Spivey, 1989) J.M. Spivey, The $Z$ Notation: A Reference Manual, Prentice-Hall, London, 1989.

(Sridharan, 1987) N.S. Sridharan, 1986 workshop on distributed AI, AI Magazine, 8(3):75-85, 1987.

(Srihari et al, 1987) S.N. Srihari, C.H. Wang, P.W. Palumbo, and J.J. Hull, Recognizing address blocks on mail pieces: specialized tools and problem solving architecture, AI Magazine, $1987(4): 25-40,1987$.

(Sriram, 1987) D. Sriram, Destiny: a model for integrated structural design, in: Proceedings First Blackboard Systems Workshop, Seattle, WA, 1987.

(Stammers, 1985) R.A. Stammers, The MXA-shell, in: M.A. Bramer, ed., Research and Development in Expert Systems, pp. 31-37. Cambridge University Press, Cambridge, UK, 1985.

(Tailor, 1988) A. Tailor, MXA - a blackboard expert system shell, in: R.S. Engelmore and A.J. Morgan, eds., Blackboard Systems, chapter 15, pp. 315-333. Addison-Wesley, Reading, MA, 1988.

(Talukdar \& Cardozo, 1987) S. Talukdar and E. Cardozo, Blackboards, lateral relations, and large scale organizations, in: Proceedings First Blackboard Systems Workshop, Seattle, WA, 1987. 
(Talukdar et al., 1988) S. Talukdar, E. Cardozo, and G. Podnar, Building large-scale software organizations, in: J.S. Kowalik and C.T. Kitzmiller, eds., Coupling Symbolic and Numerical Computing in Expert Systems, II, pp. 129-143. Elsevier Science Publisher B.V. (North-Holland), 1988.

(Terry, 1988) A. Terry, Using explicit strategic knowledge to control expert systems, in: R.S. Engelmore and A.J. Morgan, eds., Blackboard Systems, pp. 159-188. Addison-Wesley, Reading, MA, 1988.

(Tommelein et al., 1987) I.D. Tommelein, M.V. Johnson, B. HayesRoth, and R.F. Levitt, SIGHTPLAN: a blackboard expert system for construction site layout, in: J. Gero, ed., Proceedings IFIP WG5.2 Working Conference on Expert Systems in Computer-Aided Design, Sydney, 1987. Elsevier Science Publisher B.V. (North-Holland).

(Tsichritzis et al., 1987) D. Tsichritzis, E. Fiume, S. Gibbs, and O. Nierstrasz, KNOs: Knowledge acquisition, dissemination, and manipulation Objects, ACM Transactions on Office Automation Systems, 5(1):96-112, 1987.

(Tynor et al., 1987) S.D. Tynor, S.P. Roth, and J.F. Gilmore, Implementation of a generic blackboard architecture, in: Proceedings SPIE vol. 786 Applications of Artificial Intelligence $V$, pp. 116-124, 1987.

(Valkonen et al., 1987) K. Valkonen, H. Jäppinen, and A. Lehtola, Blackboard-based dependency parsing, in: Proceedings IJCAI-87, pp. 700-702, Milan, 1987.

(Velthuijsen, 1987a) H. Velthuijsen, The BLACKBIRD-project: implementation of the parallel blackboard shell BLONDIE-II (in Dutch), Memorandum 1572 DNL/87, PTT Research, Leidschendam, 1987.

(Velthuijsen, 1987b) H. Velthuijsen, Incorporating UNIX message passing in a Poplog environment, Internal Report $87 \mathrm{DNL} / 06$, PTT Research, Leidschendam, 1987. 
(Velthuijsen, 1987c) H. Velthuijsen, Manual for BLONDIE - An expert system shell with a blackboard architecture, Internal Report 87 DNL/35, PTT Research, Leidschendam, 1987.

(Velthuijsen, 1987d) H. Velthuijsen, Manual for BLONDIE-II - A parallel blackboard shell, Internal Report 87 DNL/36, PTT Research, Leidschendam, 1987.

(Velthuijsen, 1987e) H. Velthuijsen, A parallel blackboard system for robot control, in: Proceedings First Blackboard Systems Workshop, Seattle, WA, 1987.

(Velthuijsen \& Braspenning, 1990) H. Velthuijsen and P.J. Braspenning, Towards a formal model of blackboard architectures, in: Proceedings Computing Science in the Netherlands '90, pp. 469-483, Utrecht, 1990.

(Velthuijsen \& Braspenning, 1991a) H. Velthuijsen and P.J. Braspenning, A conceptual and formal study of the blackboard architecture, in: E. Moskilde, ed., Modelling and Simulation 1991, Proceedings 1991 European Simulation Multiconference (ESM '91), pp. 374-379, Copenhagen, 1991. The Society for Computer Simulation.

(Velthuijsen \& Braspenning, 1991b) H. Velthuijsen and P.J. Braspenning, Results of formalising the blackboard architecture, in: Proceedings Fifth Blackboard Systems Workshop, Anaheim, CA, 1991 .

(Velthuijsen et al, 1987a) H. Velthuijsen, B.J. Lippolt, and J.C. Vonk, A parallel blackboard architecture, in: Proceedings 3 rd International Expert Systems Conference 1987, pp. 487-493, London, 1987.

(Velthuijsen et al., 1987b) H. Velthuijsen, B.J. Lippolt, and J.C. Vonk, A parallel blackboard system for robot contral, in: Proceedings IJCAI-87, pp. 1157-1159, Milan, 1987.

(Vere, 1987) S. Vere, Planning, in: S.C. Shapiro, ed., Encyclopedia of Artificial Intelligence, volume 2, pp. 748-758, John Wiley \& Sons, New York, NY, 1987. 
(Wand, 1988) Y. Wand, A proposal for a formal model of objects, in: W. Kim and F. Lochovsky, eds., Object-Oriented Concepts, Databases, and Applications, pp. 537-559, Addison-Wesley, Reading, MA, 1988.

(Waterman, 1986) D.A. Waterman, A Guide to Expert Systems, Addison-Wesley, Reading, MA, 1986.

(Weihmayer \& Brandau, 1990) R. Weihmayer and R. Brandau, A distributed AI architecture for customer network control, in: Proceedings IEEE Global Telecommunications Conference (GLOBECOM '90), pp. 656-662, San Diego, CA, 1990.

(Werkman \& Hillman, 1989) K.J. Werkman and D.J. Hillman, Designer fabricator interpreter system, in: Proceedings 9th Workshop on Distributed Artificial Intelligence, pp. 95-111, Eastsound, WA, 1989.

(Wilkins, 1988) D.E. Wilkins, Practical Planning - Extending the Classical AI Planning Paradigm, Morgan Kaufmann Publishers, Inc., San Mateo, CA, 1988.

(Williams, 1988) M.A. Williams, Hierarchicall multi-expert signal understanding, in: R.S. Engelmore and A.J. Morgan, eds., Blackboard Systems, chapter 19, pp. 387-415. Addison-Wesley, Reading, MA, 1988.

(Winkelbauer, 1988) L. Winkelbauer, A structure for the incremental construction of coupled systems: a bridge between shallow and deep coupling, in: J.S. Kowalik and C.T. Kitzmiller, eds., Coupling Symbolic and Numerical Computing in Expert Systems, II, pp. 145-156. Elsevier Science Publisher B.V. (NorthHolland), 1988.

(Winskel, 1987) G. Winskel, Event structures, in: W. Brauer, W. Reisig, and G. Rozenberg, eds., Petri Nets: Applications and Relationships to Other Models of Concurrency - Advances in Petri Nets 1986, Part II, pp. 325-392, Springer Verlag, Berlin, 1987.

(Winston, 1984) P.H. Winston, Artificial Intelligence, Addison-Wesley, Reading, MA, second edition, 1984. 
(Yang et al., 1985) J.D. Yang, M.H. Huhns, and L.M. Stephens, An architecture for control and communication in distributed Artificial Intelligence systems, IEEE Transactions on Systems, Man, and Cybernetics, SMC-15(3):316-326, 1985. 


\section{Appendix A}

\section{Formal Description of Blackboard Architectures}

The formal definitions presented in Chapter 6 are used to describe a number of existing blackboard architectures. The reasons why these architectures were selected for being described formally have been given in Chapter 7 (see Table 7.1 on page 180). That chapter also contained an analysis based on the descriptions in this Appendix.

For each of the fourteen blackboard architectures this Appendix uses the formal definitions to describe the KSs and their life cycles, their blackboard, and their control unit. Moreover, when an architecture supports concurrency, we describe the specific form of concurrency. The descriptions of the architectures follow roughly the items specified in Tables $7.2,7.3,7.4$, and 7.5 .

Throughout the Appendix boxes are placed around the equations describing aspects of the architectures. Each box is marked DYNAMTC or $S T A T I C$ to indicate whether the symbols are to be interpreted according to their dynamic or static definitions (see also Tables 6.1 and 6.2).

The descriptions of the knowledge-source life cycles in each of the presented systems make use of the same symbols $c, s, e, r$, etc. as defined on page 174 in Chapter 6. 


\section{A.1 Hearsay-II}

The speech understanding system Hearsay-II contained the first implementation of a blackboard architecture. Hearsay-II evolved out of an earlier version, Hearsay-I, which contained some rudimentary features of a blackboard architecture, to provide a system engineering tool characterised by modularity, flexibility, and the ability to generate solutions incrementally by applying multiple sources of knowledge according to a hypothesize-and-test paradigm (Reddy et al., 1973; Lesser et al., 1975; Hayes-Roth \& Lesser, 1977; Erman et al, 1980). The application domain of Hearsay-II is speech understanding: the input is an acoustic representation of an utterance and the task is to find a semantic interpretation of the utterance.

\section{A.1.1 Knowledge sources}

Knowledge sources in Hearsay-II are condition-action pairs, but condition and action parts are both activities in themselves, subject to scheduling or selection. A KS condition does not necessarily 'belong' to a corresponding $\mathbb{K S}$ action, i.e., there is no one-to-one relation between $\mathrm{KS}$ conditions and KS actions. A meaningful mapping must be surjective, however.

Both KS conditions and KS actions are implemented as procedural bodies of LISP code. Apart from a condition part and an action part a $\mathrm{KS}$ also contains a description of the kinds of changes it makes to the blackboard. Thus:

$$
\begin{aligned}
K S & =\{\text { condition, expected-changes, action }\} \\
\text { condition } & =\{\text { precondition, LISP-code }\} \\
\text { action } & =\text { LISP-code }
\end{aligned}
$$

The condition is a procedure which is invoked when a change set (description of an event) occurs matching the precondition of that condition. Upon invocation the condition is added to the queue of pending KS conditions, together with a reference to the hypothesis, creation or 
modification of which resulted in the change set. When this pair of condition and reference is selected, the LIS P-code is executed. This code searches the blackboard for arbitrary complex patterns on the blackboard and if successful place the corresponding KS action in the queue of pending KS actions. Also two data structures for this invocation of a KS action are created: the stimulus frame and the response frame. The stimulus frame contains the set of hypotheses that matched the pattern in the condition and is thus an instantiation of condition. The response frame represents the modifications of the blackboard that this invoked KS action is expected to make and is thus an instantiation of expected-changes. Together, stimulus frame and response frame form a kind of KS activation record.

The LISP-code in the KS action may create, modify, and delete hypotheses on the blackboard, based on the hypotheses in its stimulus frame, and in turn cause the invocation of other KS conditions.

\section{A.1.2 Blackboard}

In Hearsay-II the blackboard consists of one panel, subdivided into an ordered set of levels:

$B B=$ parameter, segment, syllable, word, word-sequence, phrase, database-inter face, link ${ }^{+}$

The levels are ordered in a loose hierarchy: elements on one level represent either aggregations or abstractions of elements on a preceding level. The elements or units on a blackboard level are called hypotheses:

$$
\begin{aligned}
\text { bb-level } & =\{\text { level-id, hypathesis }\} \\
\text { hypothesis } & =\left\{\text { unit-id, interpretation, credibility-rating }\left(t_{1}, t_{2}\right)\right\} \\
\text { link } & =\text { hypothesis } 1, \text { hypothesis }
\end{aligned}
$$

A hypothesis proposes an interpretation of part of the input utterance at a level of representation corresponding with the blackboard level on 
which the hypothesis resides. Hypotheses on a blackboard level can be (partially) ordered according to time coordinates $\left(t_{1}, t_{2}\right)$, representing the beginning and the ending of that part of the input utterance a hypothesis addresses.

Each hypothesis has associated with it a credibility-rating, representing the current belief in the interpretation. Directed links are used to indicate on which other hypotheses the contents of a certain hypothesis are based.

Hypotheses on the blackboard may be created, modified, and deleted by KS actions. Procedures for "associative" matching are provided tor reading by $\mathrm{KS}$ actions and $\mathrm{KS}$ conditions.

In Hearsay-II a function is used to describe the blackboard state. This function $S$, called the state, is defined for each time vallue from the beginning to the ending of the input utterance:

$$
S(t)=\max _{h_{t}} V\left(h_{t}\right)
$$

where $h_{t}$ ranges over all hypotheses representing an interpretation containing point $t$. The value of a hypothesis, $V\left(h_{t}\right)$, is an function increasing with its level, duration, and credibility rating. The desirability of creating a new hypothesis on the blackboard can be described via the increase in state it would bring about.

\section{A.1.3 Control unit}

The control unit in Hearsay-TI executes a control cycle. In the description of the control cycle in Erman et al, 1980 the cycle starts by calculating priorities of pending activities in the scheduling queues, which contain either KS conditions or KS actions. The activity with the highest priority is selected and executed. If the activity is a KS condition the blackboard is scanned for patterns and new KS actions may be added to the scheduling queues. Two modes exist for this invocation of KS actions: either a KS action is placed in the queue for each satisfying pattern or only one $\mathrm{KS}$ action is acded with a reference to all satisfying patterns. If the activity is a KS action, the blackboard is updated as a result. Change sets (events) are created to represent relevant information about blackboard modifications, possibly triggering KS conditions which are then added to the scheduling quenes. 
In our formalisation we usually start the control cycle with the matching of events against conditions. The definition of the control unit in Hearsay-II is as follows:

$$
\begin{aligned}
& C U=\text { match }_{1} \ldots . . \text { match }_{n} . \text { sel.E. } X \\
& E X=e x_{\text {cond }} \cdot \text { sel. } E X+e x_{\text {action }} . C U+\text { stop. } \overline{\text { done }}
\end{aligned}
$$

$D Y N A M I C$

The actions match ( $n$ is the number of conditions) match the generated change sets against the $n$ specified patterns that define when to invoke the corresponding KS condition. If the match is successful the queue of pending $\mathrm{KS}$ conditions is expanded with the invoked KS condition and a. reference to the hypothesis responsible for this match.

The function sel calculates a priority for each pending activity and selects the activity with the highest priority for execution. Priority calculation for an activity is based on information about the hypotheses that invoked this activity (either a KS condition or a KS action), the expected outcome of the activity, and the global blackboard state.

When a KS condition ( $e x_{c o n d}$ ) is executed, the blackboard is searched for arbitrary complex patterns of hypotheses, forming conceptual AND/OR trees of matching prototypes. Matching prototypes are partial specifications for associative matching on the blackboard. If this search is successful the action part of a KS corresponding to this pattern is added to the queue of pending KS actions, together with a reference to the hypotheses that matched the pattern. Information about the created instantiation of a KS action is stored in two data structures, called stimulus frame and response frame.

Stimulus frame and response frame (including credibility, level, and time spanning of hypotheses represented in the two frames) are used, together with the state of the blackboard, to determine the priority of a KS instantiation. KS actions in Hearsay-II can be top-down or bottom-up and even operate within one level of representation. Control carn switch opportunistically between the different approaches.

The control unit stops when no more activities are present in the queues. Hearsay-II has a special KS that can discourage pending activities and is 
invoked when an interpretation on the highest level emerges, effectively clearing the queues and thus halting the problem-solving process.

The life cycle of a KSI in Hearsay-II is very simple:

$$
L C=\text { c.s.e.r. } \overline{\text { done }}
$$

DYNAMIC

\section{A.2 Hearsay-III}

Hearsay-III is a blackboard framework, developed as a generalisation of Hearsay-II, but with some additional characteristics. An important characteristic of Hearsay-III (Balzer et al., 1980; Erman el al., 1981) is that it is built on top of a typed relational database system, called AP3. The presence of this database and its pattern-matching language is apparent in severai aspects of the blackboard architecture.

\section{A.2.1 Knowledge sources}

Knowledge sources in Hearsay-III consist of a condition, the immediate action which is called immediate code, and a non-interruptible, procedural body in LISP. A condition is specified as a combination via AND and OR of elementary predicates which can be AP3 templates and LISP predicates. The immediate code is executed as soon as the condition of the corresponding $\mathrm{KS}$ is satisfied by units on the blackboard. The immediate code is used for binding by assigning values to attributes in a unit representing this instantiation of the $\mathbb{K S}$ on the scheduler blackboard. Such a unit is called an activation record. The immediate code also returns the appropriate unit-class for the created activation record.

$$
K S=\{\text { condition, immediate-code, LISP-code }\}
$$




\section{A.2.2 Blackboard}

The blackboard in Hearsay-III is the component where the use of AP3 is most significant. A generalised notion of levels on the blackboard is represented as a tree structure of classes. The nodes in the graph correspond to unit-classes which define types for the units (instances of a unit-class). The definition of the unit-classes and their organisation is left free, be it that the complete blackboard is subdivided into two superclasses, corresponding to a scheduling panel and a domain panel of the blackboard. The root of the class-tree is the class denoting the general concept of "unit".

Apart from this tree structure, units on the blackboard may form nodes in other graph-structures. The arcs in these graphs are labelled and are called roles. Labelling is used to represent different (component) hierarchies, thus simplifying search. Labelling is used also to administer inclusion of units in a certain hierarchy in order to be able to inhibit inclusion of the same partial solution more than once in an overall solution. Access to specific data on the blackboard can be made more efficient by using type restricted retrieval in AP3.

The definition of the blackboard in Hearsay-III is described as follows:

$$
\begin{aligned}
B B & =\left\{\text { unit-class, } \text { role }^{*}\right\} \\
\text { unit-class } & =\left\{\text { class-id },\left\{\text { unit-class }^{*}\right\},\left\{\text { class-instance }{ }^{*}\right\}\right. \\
\text { role } & =\left\{(\text { class-instance } 1, \text { class-instance })^{*}\right\}
\end{aligned}
$$

STATIC

Unit-classes define the type of their instances: units of the same unitclass have the same attributes. The top of this tree structure of unitclasses is pre-defined in Hearsay-III.

Units on the blackboard can be created and modified (not deleted). Changes in templates in the AP3 database are recorded and matched against elementary predicates in the conditions of KSs.

With each unit-class a collection of procedurally defined predicates may be associated for assessing for instance the validity of a unit with respect to a specific global constraint. These predicates are run when a unit of that class is created or marked unaccepted by a KS. If all these predicates 
run successfully, the unit becomes accepted; and unacceptable otherwise. Hearsay-III prevents KSs from being triggered by an unacceptable unit. KS instantiations having units in their unacceptable triggering contexts are not selected for execution.

Hearsay-III uses the notion of choice-sets, explicitly representing decision points on the blackboard. A choice-set is a unit, augmented to represent unresolved decisions, i.e., a set of alternatives together with a partial elaboration function or a generation function (or both) ${ }^{1}$. Ambiguity represented by a choice-set can be resolved in two different ways: by deduction (selecting one alternative and discarding all others) or by assumption (temporarily substituting one alternative for the choice-set).

\section{A.2.3 Control unit}

A change of the blackboard is matched against the elementary predicates of the conditions of KSs. If there is a match the complete pattern of the condition is matched against the contents of the blackboard. If this second match is completed successfully as well, the immediate code of the corresponding $\mathrm{KS}$ is run. The immediate code may instantiate any attribute in the activation record to be used later as a context for this instantiation of the KS. Essential is that the immediate code returns the identity of a unit-class on the scheduler blackboard. The newly created activation record is stored as an instance of that unit-class.

The default control cycle in Hearsay-III is implemented via two procedures for which (simple) default versions are supplied. These two procedures (called inner-base-scheduler and outer-base-scheduler) can be substituted, however, by arbitrarily complex alternatives. A distinction is made between (user-definable) scheduler KSs and domain KSs. A scheduler KS can return a list of activation records for (non-scheduler) KSs which the scheduler KS deems appropriate for execution.

The inner-base-scheduler is called by the outer-base-scheduler and returns a list of activation record units. The outer-base-scheduler then executes in turn all items in the returned list (if the list were empty, the problem-solving process halts). If an item is the activation record for a

\footnotetext{
"Partial elaboration and generation functions represent prescriptions for moving in the search space used in the problem-solving process.
} 
scheduler KS and returns a list of activation records, these are executed immediately, before execution of items in the original list is resumed.

Control in Hearsay-III is extremely flexible. For the selection of activation records all data on the blackboard is available. Relevant data on the KSs and their instantiations themselves can be made specific by the immediate code and is available as blackboard data as well. The way in which all this data is applied is user-definable, either by implementing dedicated scheduler KSs or by supplying another scheduler instead of the default base-schedulers. Effectively any number of KSs (scheduler or non-scheduler) can be executed between reassessment of the set of applicable KSs and their activation records. The control unit as defined by the default base scheduler can be described as follows:

$$
\begin{aligned}
C U= & \text { match }_{1} \ldots . . \text { match }_{n} . \text { inner-base-scheduler. } \\
& \left(e x_{1} \ldots . . e x_{r} . C U+\overline{\text { done }}\right)
\end{aligned}
$$

DYNAMIC

The action inner-base-scheduler implements the selection procedure. The number $r$ of KSs that are executed depends not only on the list returned directly by inner-base-scheduler, but also on the returned lists of any scheduler KSs in the original list.

We do not attempt to give a description of the life cycle of IKSs in general, as the default control cycle is completely redefinable. The default control cycle, however, can be described by the following usual and simple equation:

$$
L C=\text { c.s.e.r. } \overline{\text { done }}
$$

$D Y N A M I C$

We note that, although more than one KSI can be selected for execution at the same time and thus the problem could be solved before a specific KSI is being executed, the default control cycle does not recognise this, so a selected KSI is executed as well. 


\section{A.3 DVMT}

DVMT (Distributed Vehicle Monitoring Testbed) was developed as a tool for research in Distributed Artificial Intelligence: otherwise independent systems try to solve a shared problem cooperatively. Components achieve cooperation by making informed decisions about communication of data and tasks with other systems. In DVMT the components are complete blackboard architectures, based on the Hearsay-II architecture but with extensions for communication and more sophisticated control. The components form the nodes in a network, called a distributed problem-solving network.

This description is based on material describing versions of DVMT up to 1985 (Lesser \& Corkill, 1983; Corkill \& Lesser, 1983; Durfee et al., 1985; Durfee et al., 1987) and does not include possible later developments (mainly directed at increasing coherence in a distributed problemsolving network) such as the use of 'partial global plans'. The description is focussed on the architecture of a single node, giving some additional attention to the communication facilities needed for cooperation. Sometimes the application domain of DVMT is apparent. DVMT has been applied to monitoring vehicles (determining identity and location of vehicles) as they move around in a certain area. The input to the system is provided by acoustic signals.

\section{A.3.1 Knowledge sources}

Knowledge sources in DVMT are non-interruptible LISP procedures. They interact with the data blackboard (see below) and might occasionally write goals on the goal blackboard (explained in the next section). There are special KSs dedicated to communication with other nodes in the problem-solving network.

For each KS a condition is defined, specifying what hypotheses are needed for that KS to become applicable. The effects of KSs are represented in a table indicating goal-KS relationships. The goal-KS relationships provide the mechanism for determining the desirability of executing a specific $\mathrm{KS}$ in DVMT as opposed to the usual mechanism based on triggering in other blackboard architectures. 
KS action parts can read and write data on the data blackboard, read and write goals on the goal blackboard, and read abstractions from the abstraction blackboard.

\section{A.3.2 Blackboard}

The blackboard in DVMT is subdivided into three panels: data blackboard, goal blackboard, and abstraction blackboard. The data blackboard is subdivided into four groups of two levels. The four groups form an abstraction hierarchy and represent signals, groups, vehicles, and patterns. Each group contains two levels, ordered via an aggregation hierarchy and containing hypotheses about locations and tracks respectively.

The levels on the goal blackboard reflect the data blackboard. Units on the data blackboard are called hypotheses, while units on the goal blackboard are called goals. Goals represent an incentive to create or extend hypotheses with particular attributes on the corresponding level on the data blackboard.

Units on the abstraction blackboard are called level-time entries. Leveltime entries group together hypotheses with similar level, time, and region characteristics. Regions are meant here to indicate areas in the space monitored by the DVMT system. The abstraction blackboard is an abstracted representation of the data blackboard, ignoring details about individual. hypothesis interactions.

The structure of the blackboard can be represented by the following equations (we note that the vehicle monitoring application is apparent in this description):

$$
\begin{aligned}
B B= & \{\text { data-BB, goal-BB, abstraction-BB }\} \\
\text { data-BB }= & (\text { signal-loc, group-loc, vehicle-loc, pattern-loc), } \\
& (\text { signal-track, group-track, vehicle-track, } \\
& \text { pattern-track })
\end{aligned}
$$




$$
\begin{aligned}
\text { data-lewel }= & \left\{\text { hypothesis }{ }^{+}\right\} \\
\text {goal-BB }= & (\text { signal-loc,group-loc, vehicle-loc, pattern-loc }), \\
& (\text { signal-track, group-track, vehicle-track, } \\
& \text { pattern-track }) \\
\text { goal-level }= & \left\{\text { goal }{ }^{+}\right\} \\
\text {abstraction- } B B= & \left\{\text { level-time-entry }{ }^{+}\right\}
\end{aligned}
$$

\begin{tabular}{|c|c|c|}
\hline \multirow[t]{2}{*}{$B B$} & $=$ & $($ Data-BB $\mid$ Goal-BB $\mid$ Abstraction-BB \\
\hline & & $B B$-monitor $\mid$ C(usterer $) \backslash L$ \\
\hline \multirow[t]{2}{*}{ Data-BB } & $=$ & read-data.Data-BB+ \\
\hline & & 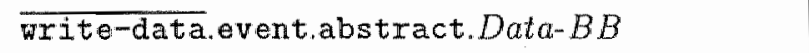 \\
\hline BB-monitor & $=$ & 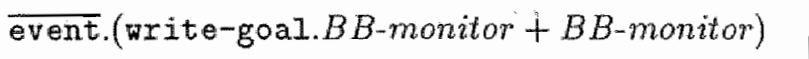 \\
\hline Goal-BB & $=$ & read-goal.Goal-BB+ write-goal.Goal-BB \\
\hline Crusterer & $=$ & $\overline{\text { abstract }}$ wite-abstraction.Clusterer \\
\hline \multirow[t]{4}{*}{ Abstraction- $B B$} & $=$ & read-abstraction. Abstraction- $B B+$ \\
\hline & & write-abstraction $A b$ straction- $B B$ \\
\hline & $=$ & \{event, abstract, write-abstraction\} \\
\hline & & $D Y N A M I C$ \\
\hline
\end{tabular}

The blackboard also participates in interactions with IKSs and several components of the control unit. For the blackboard as a process the following equations hold (Clusterer and BB-monitor are explained in the next section):

\section{A.3.3 Control unit}

The control unit in DVMT is more complex than in the previously described blackboard architectures. Unlike some other architectures (e.g., $\mathrm{BB} 1$ ) sophisticated control is not implemented via control $\mathrm{KSs}$, but via activities in the control unit. The control cycle can be described as follows. When a KS has been executed, the changes on the data blackboard are recorded by the blackboard monitor and matched against an 
event-goal table, possibly resulting in the creation of goals on the goal blackboard.

The goal-processor matches goals on the goal blackboard against a goalKS table in order to detect useful actions. Goals are matched also against a goal-subgoal table to generate subgoals when it is not possible to instantiate executable KSs satisfying the goals completely. Subgoals are added to the goal blackboard.

A clusterer includes abstractions of new hypotheses on the data blackboard in the abstraction blackboard, which in turn is monitored by the situation-recognizer in order to detect medium-term problem-solving goals.

A planner combines output from the goal-processor (identifications of KS instantiations, being short-term actions) and the situation-recognizer (specifications of medium-term tactics) together with current network organisation parameters, which specify the behaviour of the node in the distributed problem-solving network, to form prioritised plans, i.e., sequences of KS instantiations.

The control cycle can now be described by the following equation:

$$
C U=(\text { goal-processor|situation-recognizer }) \cdot \text { planner.sel.ex.CU }
$$

DYNAMIC

The actions match and sel, as defined in the general formal description of blackboard architectures in Chapter 6, are extended considerably here to be able to make better informed decisions, mainly by including information about problem-solving goals and problem-solving state abstractions. However, as can be seen, the original form is still intact.

The life cycle of a KSI in DVMT can be described as follows (the action $p$ for becoming present is included here to represent that instantiation of KSs is related to the creation of goals):

$$
L C=\text { p.e.s.e.r. } \overline{\text { done }}
$$




\section{A.3.4 Concurrency}

DVMT provides facilities for communication among the different nodes in the problem-solving network. All these facilities are completely embedded within dedicated communication KSs and do not interfere with the control unit of a node. The effects of such communications within a node are only felt indirectly via the creation of hypotheses and goals on the blackboard of that node that were received from other nodes by its communication KSs.

The action parts of the special communication KSs (called Hyp-send, Hyp-receive, etc) can be specified by their allowed interactions. The special communication $\mathrm{KS}$ s are described in the following set of equations:

$\begin{aligned} & \text { Hyp-send }= \frac{\text { read-data.Hyp-send }+ \text { int-action.Hyp-send }+}{\text { communicate.Hyp-send }} \\ & \text { Hyp-receive }= \frac{\text { communicate.Hyp-receive }+ \text { int-action.Hyp-recerve }+}{\text { Grite-data.Hyp-receive }} \\ & \text { Goal-send }= \frac{\text { read-goal.Goal-send }+ \text { int-action.Goal-send }+}{\text { communicate.Goal-send }} \\ & \text { Goal-help }= \frac{\text { write-goal.Goal-receive }}{\text { read-data.Goal-help }+ \text { int-action.Goal-help }+} \\ & \text { Goal-reply }= \frac{\text { communicate.Goal-help }}{\text { Iead-data.Goal-reply }+ \text { int-action.Goal-reply }+} \\ & \text { communicate.Goal-reply }\end{aligned}$

Port communicate represents communication between different nodes. The definitions for the KSs Goal-send and Goal-help are identical at this level of detail of the descriptions. The semantics are different, however. Goal-send merely informs other nodes of created goals, while Goal-help requests for assistance by other nodes to achieve the goal specified in the request. Goal-reply returns hypotheses that satisfy such a transmitted goal. 


\section{A.4 HASP/SIAP}

HASP and SIAP are two successive implementations of a blackboard system for sonar interpretation (Nii et al, 1988). The domain is the recognition and localisation of vessels in an ocean area, via interpretation of sonar data. The sonar data is preprocessed and then represented in the HASP/SIAP system as a collection of lines. SIAP tried to include more low-level processing than HASP did. The architectures of the two systems are sufficiently similar to discuss them as one in this section.

HASP/SIAP differs from other blackboard architectures mainly regarding the type of task of its problem domain, namely a monitoring task. The problem is to maintain a correct interpretation of incoming sonar data from a certain ocean area over a period of time. This means that the HASP/SIAP system has to include some sense of time. Also, the interpretation of the real-world as represented on the blackboard necessitates more careful treatment of competing or conflicting entries on the blackboard.

\section{A.4.1 Knowledge sources}

KSs in HASP/SIAP are hierarchically organised. In the implementation as described in Nii et al., 1988 there is one strategy KS, two KSactivators, called Event-driver and Expectation-driver respectively, and a number of specialist KSs. Specialist KSs perform the actuall application oriented processing in the system. The other KSs are used for control. The strategy $K S$ reacts to so called clock-events, which form an implementation of timers in HASP/SIAP. The KS Event-driver reacts to ordinary events. The Expectation-driver reacts to a third kind of events, called problems. Problems are used as a suspension mechanism: when a specialist $K S$ discovers it has not enough information it can post a problem specifying the missing data and the activities to perform when the missing data is derived later on. These activities are activated by the Expectation-driver when the missing data becomes available. This mechanism can be used as a form of self-suspension.

Apart from this self-suspension mechanism, KSs are non-interruptible. The action parts of KSs can be heterogeneous: in the HASP/SIAP im- 
plementation some knowledge sources ase rules, while others use framebased representations or procedures.

The HASP/SIAP architecture does not provide for multiple instantiations of KSs: the control component first determines all expired clockevents and processes the appropriate KSs. Then the Expectation-driver tries to resolve all pending problems by matching these with newly occurred events. Finally, the Event-driver selects events and executes KSs that can process the selected events. Selection is thus based on events, not on the KSs that can process selected events. It was expected that no information about KSs would be needed for control. The only information associated with $\mathrm{KSs}$ is a description of the (ordinary) events.

$$
\begin{aligned}
K S & =\{\text { trigger }, \text { action-part }\} \\
\text { trigger } & =\left\{\text { event-abstraction }{ }^{+}\right\} \\
\text {action-part } & =\left\{\text { rule }^{*}, \text { frame }^{*},[\text { procedure }]\right\}
\end{aligned}
$$

STATIC

\section{A.4.2 Blackboard}

The contents of blackboard in HASP/SIAP forms a model of the ocean area under observation as it is currently interpreted by the system, hence the name Current Best Hypothesis, or CBH. The CBH is subdivided into different levels, called levels of analysis, which are strictly ordered. The resulting hierarchy is not a strict abstraction or aggregation hierarchy, but is based on different levels of interpretation during the problem-solving process. There are five levels of analysis: lines, harmonics, sources, vessels, and situation board. Data on the levels are typed and called hypothesis elements. Hypothesis elements contain an informal confidence weight for each of their property values.

HASP/SIAP uses directed links between the hypothesis elements. Links from an element on one level of analysis to an element on a higher (according to the strict ordering) level of analysis are called reduction links, while links in the other direction are called expectation links. The links coincide with $\mathrm{KS}$ inferences, which generally operate from one level of analysis to another level. 


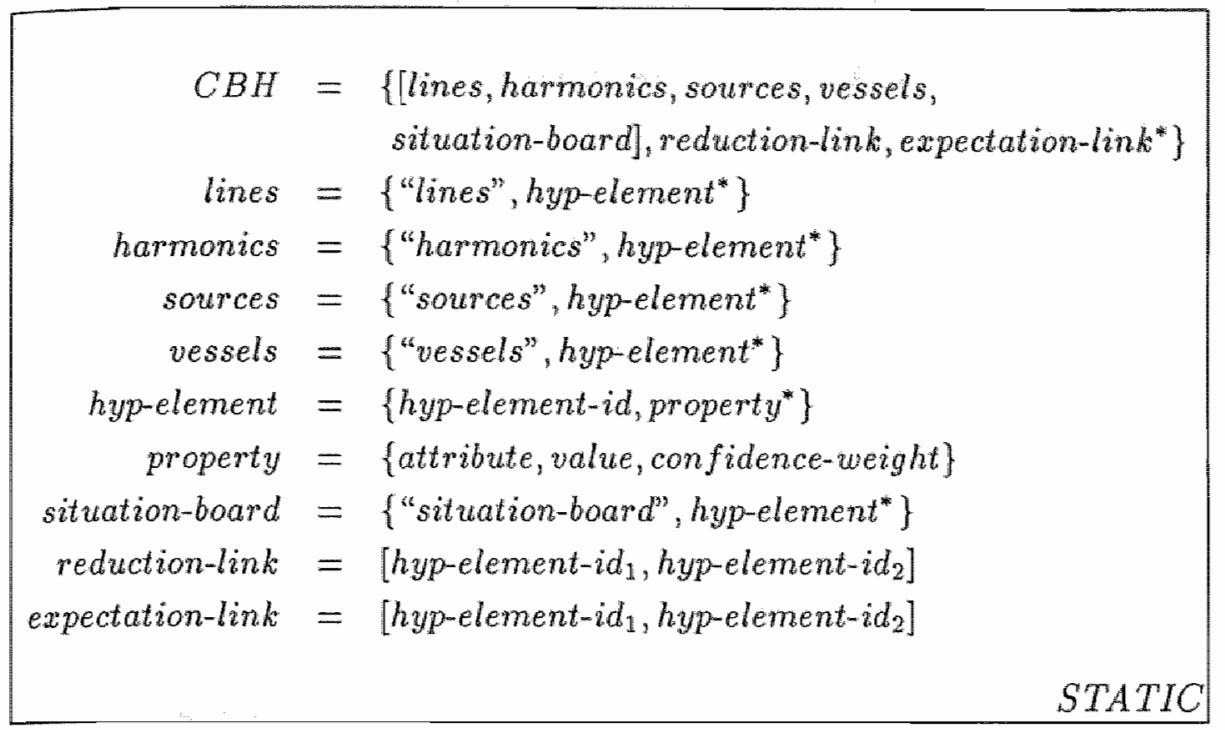

The CBH allows for the usual interactions: adding elements, modifying existing hypothesis elements, and reading information. There exist three kinds of events. Clock-events and problems are created directly by KSs. Clock-events represent time-points where certain actions have to be taken. A clock-event contains a time-point and a $\mathbb{K S}$ name. Problems are dynamically created and represent the need for a specific event that has to occur before a certain activity can be executed. A problem contains an event(-type) and KS name. Events of the third kind (ordinary events) contain an abstracted description of a blackboard modification. These events are created indirectly as a result of blackboard modifications by KSs.

\section{A.4.3 Control unit}

The control unit in HASP/SIAP executes a control cycle. When the cycle starts, the strategy $K S$ checks which clock-events need to be processed at this time in the problem-solving process. The strategy KS activates in turn the KSs ( $K S$-activators or specialist KSs) able to process the expiring clock-events. Next, the Expectation-driver is called to match pending problems with occurred ordinary events. If a problem matches with an event, the corresponding KSs are executed. Finally, the Eventdriver is called to select an event or group of events. The Event-driver 
then calls the specialist $K S S$ able to process the selected event(s). The cycle is repeated until there are no more events.

$$
\begin{aligned}
& C U=\text { natch-clock-events.ex } x_{1} \ldots e x_{m} \cdot \text { match-problems.ex } x_{1} \ldots e x_{p} . \\
& \text { match-events.ex } x_{1} \ldots \text { ex e.CU }
\end{aligned}
$$

DYNAMIC

The life cycle of instantiations of specialist KSs in HASP is slightly more complex than in most other examined blackboard architectures, due to the mechanism that allows KSs to specify problems as a form of selfsuspension. The description is as follows (as HASP does not recognise KS instantiations as separate objects, the life cycle does not include removal):

$$
L C=c \cdot e \cdot(i \cdot L C+\overline{\text { done }})
$$

DYNAMIC

We note that the life cycle does not include selection. In HASP/SIAP selection is aimed at event selection. Once an event is selected, related KSIs are all automatically executed.

\section{A.5 AGE}

AGE (an acronym for "Attempt the Generalize") forms an early example of a blackboard framework. It was developed as a tool box for building knowledge-based systems and aimed at compiling and making available emerging AI techniques. As a consequence AGE (Nii \& Aiello, 1979; Aiello, 1983; Nii, 1985) is equipped with a front end, which aids with the development of knowledge-based systems in AGE, and a collection of different AI techniques, from which selections can be made for the implementation of an application system. Some characteristics of HASP/SIAP can be recognised in the organisation of AGE. 


\section{A.5.1 Knowledge sources}

KSs in $\mathrm{AGE}$ are condition-action pairs similar to KSs in most other blackboard architectures. The action part of a KS in AGE consists of a set of rules. The way in which the rules in the action part of any KS are used can be specified by an application builder. AGE provides different evaluators for the rules, such as forward chaining and backward chaining, single and multiple hit (selecting one or all applicable rules, respectively), etc. Evaluators that are not provided can be implemented by an application builder.

The condition of a KS is specified by a list of events called preconditions. In AGE there are three kinds of steps processing different kinds of events: ordinary events, expectations, and goals. Events (as usual, representing modifications of the blackboard) are matched with the condition. Expectations represent expected blackboard items or values. Activities can be associated with an expectation. These activities are executed when the expected blackboard item or value indeed appears on the blackboard. Goals represent intentions to create certain blackboard items and are used in goal-directed reasoning. Goals are matched with the RHSs of rules in the action part of a KS. The RHSs of rules are responsible for generating events (indirectly) and expectations and goals (directly). All these items are represented as labels and placed on an event-list, expectation-list, and goal-list, respectively.

AGE does not provide for blackboard state matching in the ISS conditions. Variable binding can only occur inside the LHSs of the rules in the action parts of KSs. AGE does not represent KS instantiations: the control unit selects first an interesting event and then one of the $\mathbb{K} S \mathrm{Ss}$ with a matching precondition.

AGE uses descriptions of the KSs, which contain the following information: condition in the form of a list of events; a list of hypothesis levels involved in its processing; a list of links the KS will generate; specification of single or multiple hit: and a variable binding facility (variables are bound by the LHSs of the rules in the KS action parts for use by the RHSs).

KSs can thus be represented as follows. 


$$
\begin{aligned}
K S= & \left\{\text { condition, } \left\{\text { hypothesis-level }{ }^{+},\{\text {link" }\}\right.\right. \\
& \text { hit-strategy, binding }\} \\
\text { condition }= & \left\{\text { event }{ }^{+}\right\} \\
\text {hit-strategy }= & \text { single" } \mid \text { "multiple" }
\end{aligned}
$$

STATIC

\section{A.5.2 Blackboard}

In AGE, levels are seen as forming a hierarchy. Different (related) hierarchies can be represented as different panels of the blackboard. The number of both panels and levels on panels may vary. The items on the blackboard levels are called hypothesis elements and are implemented as sets of attribute-value pairs. All elements on the same blackboard level have the same attributes. The name, or identifier, of a hypothesis element can be prespecified by the application builder. There are two different kinds of directed links in AGE (similar to HASP/SIAP): reduction-links to indicate support from below (according to the hierarchy) and expectation-links to indicate support from above.

$$
\begin{aligned}
B B & =\left\{\text { panel }+, \text { reduction-lin } k^{*}, \text { expectation-lin } k^{*}\right\} \\
\text { panel } & =[\text { level } \\
\text { level } & =\{\text { level-name, hyp-element } \\
\text { hyp-element } & =\left\{\text { name, }\left\{\text { altribute, } \text { value }^{*}\right\}\right. \\
\text { reduction-link } & =\left[\text { hyp-element }{ }_{1}, \text { hyp-element }_{2}\right] \\
\text { expectation-link } & =\left[\text { hyp-element }_{1}, \text { hyp-element }_{2}\right]
\end{aligned}
$$

Hypothesis elements as well as the links between hypothesis elements can be created, modified, and read by KSs. Manipulation of links can be used for specifying events, expectations, or goals analogously to manipulation of hypothesis elements.

AGE provides at least one mechanism for reasoning with uncertainty on the blackboard., i.e., the calculus of Certainty Factors (Shortliffe \& Buchanan, 1984). 


\section{A.5.3 Control unit}

The selection of a $\mathrm{KS}$ for execution proceeds in a sequence of stages. First, the kind of step is chosen (step being the processing of either an event, an expectation, or a goal). Next, one item from the appropriate list (event-list, expectation-list, or goal-list) is chosen. Then the selected item is matched with the KSs (matching occurs via look-up tables) and one of the matching KSs is selected for execution. The control cycle is terminated when user-specified conditions appear on the blackboard.

The use of events, expectations, and goals provides reasonable control flexibility, allowing intermittent application of top-down and bottom-up processing or event-driven and goal-directed processing.

$$
C U=\operatorname{stop} \cdot(\overline{\text { done }}+\text { sel-step.sel-item.match-item.sel-action.ex.CU })
$$

$D Y N A M I C$

The life cycle of a KS in AGE resembles strongly possible life cycles in HASP/SIAP. Again the architecture recognises a kind of event (expectation) for which a trigger can be defined dynamically. An expectation can be used a means for self-suspension. Also analogously to HASP/SIAP, selection is restricted to events and does not apply a separate selection action after an appropriate $\mathrm{KS}$ has been identified. Thus the life cycle of a $\mathrm{KS}$ in $\mathrm{AGE}$ can be described by the same equation as for HASP/SIAP:

$$
L C=c \cdot e \cdot(i \cdot L C+\overline{\text { done }})
$$

$D Y N A M I C$

\section{A.6 CAGE}

CAGE is a parallel blackboard framework, i.e., it has a shared memory blackboard, while KSs (and activities within KSs) can be executed in parallel. CAGE (Nii, 1986c; Aiello, 1986) was developed alongside POLIGON as part of the Advanced Architectures Project (Rice, 1989a). Both blackboard frameworks were designed as part of a study of the possibilities of the blackboard architecture for concurrent processing. 
POLIGON, on the one hand, is an inherently concurrent framework, where users must explicitly state synchronisation points. CAGE, on the other hand, invites the user to specify what actions are to be executed in parallel. CAGE (Concurrent AGE) can best be seen as a shared-memory parallelisation effort of the AGE system (Nii \& Aiello, 1979).

\section{A.6.1 Knowledge sources}

Knowledge sources in CAGE are similar to KSs in AGE, but with one notable exception: the possibility of executing $\mathrm{KSs}$, rules in $\mathrm{KSs}$, and parts of rules concurrently. It is left to the user to specify what items are to be executed in parallel. Like in AGE the action part is a set of rulles. The same flexibility for evaluating rules applies as for AGE. The added flexibility of concurrency enables parallel evaluation of rules, clauses, and statements within clauses (implemented in Qlambda - a parallel LISP version). Additionally, the action parts of applicable rules may be executed concurrently, with or without synchronisation upon beginning and ending of execution.

For each KS in CAGE the following items must be specified (apart from the rules): name; condition in the form of a list of events (preconditions); a list of classes that will be accessed by the KS; lisi of links that might be generated; hit-strategy, a specification of single or multiple hit; a variable binding facility, which is used by the LHSs of the rules in order to avoid reevaluation of the same predicates for different rules; the order in which rules should be evaluated; and a definition of the LHS evaluator. Preconditions are tokens that should equal events (also represented via tokens). The RHS of a rule may specify an expectation which implements a dynamic trigger: as soon as the event specified by an expectation occurs, the corresponding activities (defined by the same RHS that posted the expectation) are executed.

$$
\begin{aligned}
K S= & \{K S \text {-descr, KS-action }\} \\
K S \text {-deser }= & \left\{\text { name, }\left\{\text { precondition }{ }^{+}\right\},\left\{\text {class-id }^{+}\right\},\{\text {link }\right. \\
& \text { hit-strategy, binding, order, LHS-evaluator }\}
\end{aligned}
$$

(continued on next page)

$S T A T I C$ 


$$
\begin{aligned}
K S \text {-action } & =\{\text { rule } \\
\text { rule } & =\{L H S, R H S\}
\end{aligned}
$$

Similarly to AGE, there is not a separate representation of $\mathrm{KS}$ instantiations in $\mathrm{CAGE}$. When an event is chosen for processing, a (set of) KS(s) matching the event is selected and immediately executed. Instantiations of not selected KSs are not retained for future execution.

\section{A.6.2 Blackboard}

The blackboard in CAGE is structured via classes. There is no obligatory hierarchy. Instances of the classes are called nodes, which form the basic elements on the blackboard. Nodes are sets of attribute-value pairs. Each node in a class has the same attributes, possibly with default values. Links between nodes have a name, are directed, and may have associated with them the name of an opposite link. Links are implemented as atitributes.

$$
\begin{aligned}
B B & =\left\{\text { class }^{+}\right\} \\
\text {class } & =\left\{\text { class }-i d, \text { node }^{*}\right\} \\
\text { node } & =\left\{\text { node-id },(\text { attribute }, \text { value })^{*}\right\}
\end{aligned}
$$

The user must specify tokens to be associated with a specific modification of the blackboard. These tokens serve as events and can be matched with the preconditions of KSs. When comparable events occur, a standard mechanism is provided which can be used for discarding all but one of those comparable events.

Remarkably, no mechanism is provided for maintaining consistency of data on the blackboard. One should be warned, however, that rules may be executed concurrently, thus accessing the blackboard simultaneously. 


\section{A.6.3 Control unit}

The control unit in CAGE is more flexible than in AGE. This is not only due to the added possibilities for parallelism: other steps in the control cycle are also more flexible.

In AGE the control cycle started with the selection of a kind of step (cvent, expectation, and goal). Then one step-item from the chosen steplist was chosen and only one KS that processed the chosen step-item was selected for execution.

Although CAGE does not include goals, the AGE control cycle is only a special case of the CAGE control cycle. In CAGE any number of step-items can be selected for processing and any number of KSs to do this processing. The selected KSs can be executed sequentially and in parallel. Concurrent execution of KSs can proceed synchronously as well as asynchronously: either the control unit executes all selected KSs and waits until all KSs have terminated before continuing to select a new collection of KSs or the control unit executes KSs as soon as an event occurs enabling them. Execution of KSs can not be interrupted, although execution of RHSs of concurrently evaluated rules in one KS may be synchronised. This leads to any of the following three definitions (depending on the specific extent of concurrency and synchronisation).

$$
C U=\text { stop. }
$$

$\left(\overline{\text { done }}+\right.$ sel-step.sel-item.match-item.sel-action.ex $x_{1} \ldots$ ex $\left.x_{r} . C U\right)$

$D Y N A M I C$

$$
\begin{aligned}
C U= & \text { stop } \cdot(\overline{\text { done }}+\text { sel-step.sel-item.match-item.sel-action. } \\
& \left.\left(e x_{1}|\ldots| e x_{r}\right) \cdot C U\right)
\end{aligned}
$$

DYNAMIC

$$
\begin{aligned}
C U & =C U \mid\left(\overline{\text { event. }}\left(K S-\text { action }_{1}|\ldots| K S \text {-action }_{n(i)}\right)\right) \\
K S \text {-action } & =\text { int-action.KS-action },+ \text { event.KS-action }+\overline{\text { done }}
\end{aligned}
$$

$D Y N A M I C$ 
The applicability of KSs is determined by matching preconditions of KSs with events (tokens). The control cycle stops when user-defined conditions occur on the blackboard or in the step-lists (event-list or expectation-list). The use of expectations provides a means for dynamic definition of triggers and associated actions and could be used also for delayed execution of (parts of) KS action parts (self-suspension).

The life cycle of KSIs in CAGE combines aspects of HASP/SLAP and $\mathrm{AGE}$ with a characteristic that stems from the ability to evaluate a condition on the contents of the blackboard, which decides whether a solution has been found. The consequence is that a selected KS is not executed when a solution has been found previously. This results in the following description of the life cycle for a $\mathrm{KS}$ :

$$
L C=\operatorname{css}(\text { e. }(i \cdot L C+\overline{\text { done }})+\overline{\text { done }})
$$

DYNAMIC

\section{A.6.4 Concurrency}

A number of different activities can be executed concurrently in CAGE. These activities are: $K S s$, rule-LHSs, LHS-clauses, code in clauses, and rule-RHSs. All options are user-definable. Strictly, only the parallel execution of KSs is of importance from an architectural point of view; KSs are merely seen as otherwise independent programs, which are only interesting in the way they interact with their environment. At the architectural level of detail the allowed concurrency has been specified in Section A.6.3 and was represented by the symbol "l".

\section{A.7 POLIGON}

The architecture of the POLIGON framework diverges significantly from usual blackboard architectures, as can be seen from its description in this section. Yet, it has been labelled as a blackboard architecture and it contains indeed some essential characteristics of blackboard architectures. POLIGON (Nii, 1986c; Rice, 1986; Rice, 1989b; Rice, 1989c) is 
aimed at running on a fine-grain, distributed memory, concurrent hardware platform. POLIGON was deweloped within the same Advanced Architectures Project (Rice, 1989a) as CAGE. The developments of the CAGE and POLIGON architectures were based on a number of opposing design assumptions, the most important of which are: shared memory in CAGE versus distributed memory in POLIGON and user-defined parallelism in CAGE versus user-defined sequentialisation in POLIGON. This resulted for POLIGON in an architecture, where the elements on the blackboard are distributed and actions are associated with these elements and executed immediately when associated elements are accessed in a prespecified way.

\section{A.7.1 Knowledge sources}

POLIGON does use the concept of KSs, but only as an abstraction mechanism; a means for clustering related rules, thus a KS is nothing more than a name for a set of rules. In POLIGON evaluation of rules form the basic steps in the problem-solving process. The conditions of rules are directly associated with items on the blackboard in a demonlike fashion. As soon as the condition is fulfilled, the RHS or action part of the rule is executed. As a result, other blackboard items may be changed, thus triggering other rules. Hence, the condition is in fact a trigger.

The conditions may be specified in terms of creation of items on the blackboard, modification of attributes of items on the blackboard, a semaphore being waved at an attribute (an event not related to an actual change on the blackboard), or time-points according to a real-time clock.

The action parts (RHSs) of rules can create and modify items on the blackboard, wave a semaphore at an attribute, or post an expectation. An expectation dynamically specifies a trigger. When an event matching an expectation occurs, the actions are executed specified by the part of the RHS that posted the expectation.

$$
\begin{aligned}
K S & =\left\{\text { name, } \text { rule }^{+}\right\} \\
\text {rule } & =\left\{\text { trigger }^{+}, \text {RHS }\right\}
\end{aligned}
$$




\section{A.7.2 Blackboard}

The blackboard in POLIGON, though actually distributed, is the component that probably best resembles other existing blackboard architectures. In POLIGON the blackboard is organised as a hierarchy of classes. Instances of a class are called nodes and correspond to units. All instances of the same class have the same record-like structure with the same attributes and possibly multiple values.

Classes can be organised in a hierarchy of superclasses (defining characteristics of instances of classes) and/or metaclasses (defining characteristics for classes themselves). As classes are instances of metaclasses, they are represented also on the blackboard as nodes. Links, or relations, between nodes are themselves instances of a (link) class and represented as nodes on the blackboard. As a result, links can be used in the same way as other nodes for triggering rules. Some relations between nodes are provided by POLIGON: instance-of, part-of, and subclass-of. Other relations (links) can be defined by the user. The class-relations can be used for inheritance of attribute values.

\section{A.7.3 Control unit}

In POLIGON there is no selection of actions: rules are triggered in a demon-like fashion and immediately (at least in a conceptual sense) executed. Control is not an issue in POLIGON. The only (inherent) control present in an application based on POLIGON can be found in the serialisation as induced by subsequent triggers and actions.

The life cycle of the basic activities in POLIGON can thus be described via the simple equation:

$$
L C=\text { p.c.e.LC }
$$

DYNAMIC

\section{A.7.4 Concurrency}

POLIGON supports fine-grain parallelism. The smallest undividable entities are nodes and rules. Whether this granularity is actually achieved 
depends on both the granularity of the available hardware and the number of entities needed in a certain application. However, such considerations are hidden from the user.

$$
\begin{aligned}
\text { POLIGON } & =\text { POLIGON } \mid\left(\overline{\text { event }_{1}}\left(\text { rule }_{1}|\ldots| \text { rule }_{n_{t}}\right)\right) \\
\text { rule }_{r} & =\text { int-action.rule } \\
+ \text { event.rule } & +\overline{\text { done }}
\end{aligned}
$$

DYNAMIC

\section{A.8 BB1}

BB1 was developed as a generalisation of the blackboard-based errandplanning system OPM, which was used to illustrate the validity of the blackboard architecture as a cognitive model for planning (Hayes-Roth \& Hayes-Roth, 1979). An approximation of the higher (abstraction) levels of the planning task in OPM are retained as a control component in BB1, including several control KSs and a control blackboard. Consequently, in BB1 (Hayes-Roth, 1984; Hayes-Roth, 1985; Hayes-Roth \& Hewett, 1985) much attention is given to an explicit representation of control.

\section{A.8.1 Knowledge sources}

The action parts of KSs in BB1 consist of sets of rules. Associated with each $K S$ is a collection of attribute-value pairs, called $K S$ descriptor, in which relevant attributes of that KS are represented. A KS descriptor contains slots for: the name of the KKS; a description of the KS; a condition for the $\mathrm{KS}$, consisting of an event-based trigger and a state-based precondition; a list of variables (condition-vars) that can be assigned values when the trigger or precondition is evaluated; and a list of variables (scheduling-vars) that can be used for KS selection purposes. This leads to the following description of KSs in BB1:

$$
\begin{aligned}
K S & =\{\text { descriptor, action-part }\} \\
\text { descriptor } & =\{\text { KS-name, description, condition, }
\end{aligned}
$$

(continued on next page)

$S T A T I C$ 


$$
\begin{aligned}
& \text { condition-vars, scheduling-vars }\} \\
\text { condition } & =\{\text { trigger, precondition }\} \\
\text { features } & =\left\{(\text { attribute, value })^{*}\right\} \\
\text { action-part } & =\{\text { rule }
\end{aligned}
$$

Both trigger and precondition can be arbitrarily complex combinations of (LISP) predicates. The evaluation of a trigger is the execution of a procedure, with one event as parameter and a Boolean as result (although a trigger itself can be a combination of predicates, no combinations of events are used). When the evaluation of a trigger is successful, a data structure representing this instantiation of the corresponding KS is created, called a knowledge source activation record or KSA $R$ for short. The evaluation of a precondition has no parameter, but returns a Boolean similar to a trigger. Evaluation of both trigger and precondition may result in binding of variables. These variables are defined explicitly in the attribute condition-vars.

A KSAR contains similar information to the $\mathrm{KS}$ descriptor, but also includes special instantiation information. The contents of a KSAR are: an identification, called name; $K S$-name of the corresponding KS; triggering-cycle specifying in which cycle of the control loop this instantiation was created; a description of the triggering-event; the slot triggering-decision indicating the blackboard element where the triggering event occurred; the preconditions and current associated bindings in precondition-value; bindings of condition-vars for use in the action part in condition-values; bindings of scheduling-vars for use by the control unit in scheduling-values; ratings specifying the current ratings in light of operational heuristics; and a priority.

A distinction is made between triggered and invocable KSARs. A KSAR is called invocable when its precondition has been evaluated successfully (and hence its complete condition has been satisfied; a KSAR is only created after it has been triggered). A triggered KSAR is a KSAR which is not invocable.

A description of KSARs is as follows: 


\section{$K S A R=$ \{name, KS-name, triggering-cycle, triggering-event, triggering-decision, precondition-values, condition-values, scheduling-values, ratings, priority\}}

STATIC

The slots in a KSAR are filled at different moments during the problemsolving process. Some are filled upon instantiation of the KSAR (these are name, KS-name, triggering-cycle, triggering event, and triggeringdecision). The slot precondition-values is filled with the precondition predicates upon instantiation, but variables used inside the precondition are bound during evaluation. As preconditions may be evaluated more than once, the values in precondition-values may be updated as well. The slot condition-vars is updated with trigger information upon KSAR creation and with precondition information when the precondition has been evaluated. Scheduling-values are instantiated directly from the corresponding values in the $\mathrm{KS}$ descriptor (scheduling-vars-slot) or computed by applying functions which are specified in the schedulingvars-slot in the KS descriptor and which are executed upon instantiation of the KSAR. Finally, the ratings and the priority are re-computed during each cycle of the control loop.

\section{A.8.2 Blackboard}

The blackboard in BB1 consists of two panels: one for control data and one for domain data, called control blackboard and domain blackboard, respectively. Each blackboard panel consists of an ordered set of blackboard levels. The levels on the control panel are predefined and are called problem, strategy, focus, policy, to-do-set, and chosen-action. The semantics of these levels are partly derived from the cognitive model of planning as described in (Hayes-Roth \& Hayes-Roth, 1979). For each blackboard level an ordered list of attributes must be specified (together with optional initial values). These attributes define the structure of elements (units) on that level. Units are sets of attribute-value pairs. BB1 does not explicitly provide for the use of links (although they can be defined as unit attributes).

The static definition of the blackboard in BB1 is thus: 


$$
\begin{aligned}
B B= & \{\text { control, domain }\} \\
\text { control }= & \{\text { "control", problem, strategy, focus, } \\
& \text { policy,to-do-set, chosen-action }\} \\
\text { domain }= & \{\text { "domain", level }\} \\
\text { level }= & \{\text { level-id, unit }\} \\
\text { problem }= & \{\text { "problem", unit" }\} \\
\text { strategy }= & \{\text { "strategy", unit }\} \\
\text { focus }= & \{\text { "focus", unit" }\} \\
\text { policy }= & \{\text { "policy", unit" }\} \\
\text { to-do-set }= & \{\text { "to-do-set", unit" }\} \\
\text { chosen-action }= & \{\text { "chosen-action", unit" }\} \\
\text { unit } & \left.=\{\text { unit-id, (attribute, value })^{*}\right\}
\end{aligned}
$$

The level problem is used to represent the problem currently being solved. The control levels strategy, focus, and policy are used to represent current operating heuristics. The level to-do-set lists all not yet executed KSARs, distinguishing between triggered and invocable KSARs. Finally, the level chosen-action contains a reference to the action last selected for execution.

When a modification occurs on the blackboard, an event is created. An event specifies the kind of change (created, modified, or superseded) and the identification of the unit involved in the change. The description of an event is therefore:

$$
\begin{aligned}
\text { event } & =\{\text { kind-of-change, unit-id }\} \\
\text { kind-of-change } & =\text { "created"|"modified"|"superseded" }
\end{aligned}
$$

\section{A.8.3 Control unit}

Although control is an important issue in $\mathrm{BB} 1$, the control unit itself is reasonably simple; more control sophistication can be obtained by 
applying control KSs. BB1 provides a number of predefined, generic control KSs. Thus, great flexibility of control can be achieved.

The control unit in BB1 performs a loop, called basic control loop. The basic control loop is implemented in BB1 as a succession of three stages. The loop starts with the first stage after the completion of execution of a KS. This stage (Update-to-do-set) updates the control blackboard level to-do-set. First the just executed $\mathrm{KSAR}^{2}$ is removed from the list on the level to-do-set. All events that have been created as a result of actions by the just completed KS are then given as input for evaluation of the triggers of all KSs. In those cases where the trigger evaluated successfully a KSAR is created for the corresponding pair of event and KS and added to the list of triggered KSARs on the level to-do-set. Next, the preconditions of all triggered KSARs are evaluated; successfully evaluated preconditions result in a move of the KSAR from the list of triggered KSARs to the list of invocable KSARs. Then for all KSARs (triggered and invocable) its ratings against current operative heuristics (as represented in the blackboard levels strategy, focus, and policy) is calculated. Finally, a priority is calculated for all KSARs, based on the ratings and a rule for integrating these ratings.

The second stage (Choose-KSAR) selects one of the invocable KSARs to be executed next (this is not necessarily the KSAR with the highest priority). The precondition of the selected KSAR is then re-evaluated. When the precondition still evaluates successfully, this KSAR is executed in the next stage (Interpret-KSAR). Otherwise, another KSAR is proposed.

In the third stage, execution of the action part of a KS results in an evaluation of the rules in the action part. The left-hand-sides of the rules can read the contents of the blackboard, the right-hand-side may modify the contents of the blackboard.

The user can interfere with the system at two moments during the basic control loop. BB1 provides an opportunity for users to inspect the state of the problem-solving process (blackboard state and KSs) at the end of the first stage in the basic control loop. During the second stage the user may evaluate the choice for the chosen next action and may overrule the selection even.

\footnotetext{
${ }^{2}$ Executing a KSAR means that the action part of the corresponding KS is executed with as its inputs the bound variables represented by that KSAR.
} 
The life cycle for a KSI (KSAR) includes a test, determining whether this KSI is still applicable when it is eventually selected for execution. This leads to the following description:

$$
\begin{aligned}
L C & =p \cdot L \\
L & =\operatorname{css}(\text { e.r. } \overline{\text { done }}+L)
\end{aligned}
$$

DYNAMIC

\section{A.9 AGORA}

The programming environment AGORA was developed at CarnegieMellon University - the same university where Hearsay-II evolved. Although AGORA is a general environment (Adams \& Bisiani, 1986; Bisiani, 1986; Bisiani et al., 1987; Bisiani \& Forin, 1988), it was developed with the domain of speech understanding in mind. As the incentive for building AGORA is based on the same application domain as Hearsay-II and both systems were developed at the same university (although by different researchers), it is interesting to see in which ways AGORA extends the ideas present in Hearsay-II.

AGORA is aimed at providing a programming environment on a heterogeneous software and hardware platform, where concurrency is explicitly supported. AGORA consists of several layers. The lowest layer, the bottom layer, is a network of heterogeneous processors. This network interfaces with an operating system, called MACH layer, which supports the heterogeneous and distributed assembly of machines. The next level is called the parallel virtual machine and provides the elementary building blocks for developing applications. System definitions on the level of the virtual machine are independent of the incidental configuration of hardware at the lowest layer. Higher layers include frameworks, tools, application, user interface etc. The parallel virtual machine level coincides best with the level of specification of blackboard architectures, as it is defined in terms of, e.g., knowledge sources and element cliques, which perform a role similar to knowledge sources and blackboard levels, respectively, in blackboard architectures. In AGORA processing is subdivided in knowledge sources and communication between knowledge 
sources is organised via data structures clearly resembling blackboard levels. Information in these data-structures, called element cliques, are used to determine applicability of KSs.

\section{A.9.1 Knowledge sources}

Instantiations ${ }^{3}$ of KSs in AGORA are permanent objects: from the moment an instantiation is created onwards it will continue to exist. A KS instantiation may execute different functions. An activation-pattern specifies in which situation what function, called entry point, is to be executed.

The definition of a KS in AGORA contains the following items: type definitions of the cliques employed by the KS; a name for this KS; a declaration of the parameters used by the KS; and entry points, which are the functions the KS can perform, together with the activation-patterns that specify when a specific function should be executed. Activationpatterns can be (dynamically) specified by using combinations of arrival events (data-structures representing the arrival of a new element in a clique) and values in clique data. Combinations may include different cliques. Knowledge sources in AGORA can be heterogeneous: different programming languages (notably LISP and $C$ ) can be used for implementing the functions. A KS is thus defined as follows:

$$
\begin{aligned}
K S= & \left\{\text { name, clique-definition }{ }^{*}\right. \text {, parameter-definition } \\
& \text { entry-point } \left.{ }^{+}\right\} \\
\text {entry-point }= & \{\text { activation-pattern, function }\} \\
\text { function }= & \text { LISP-code } \mid \text { C-code }
\end{aligned}
$$

Dynamically, KS instantiations act as follows:

\footnotetext{
${ }^{3}$ Instantiations of $\mathrm{KS}$ in AGORA are actually processes executing a copy of the corresponding $\mathrm{KS}$. "The term "instantiation" is used here differently from the way it is used in other descriptions where it represents a pair consisting of a KS and a context.
} 


$$
\begin{aligned}
& K S I_{j}=\operatorname{match}_{j} \\
& \left(E^{n}-\text { point }_{j_{1}} ; K S I_{j}+\ldots+E_{r-p_{0 i n t} t_{\mathrm{r}(j)}} ; K S I_{j}+K S I_{j}\right)
\end{aligned}
$$

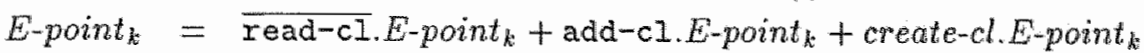

$$
\begin{aligned}
& + \text { share-cl. E-point }+ \text { int-action. E-point }+ \text { done }
\end{aligned}
$$

DYNAMIC

\section{A.9.2 Blackboard}

AGORA supports objects for communication between knowledge sources resembling the blackboard in blackboard architectures. These objects are called cliques. The cliques resemble blackboard levels. Information on the blackboard is stored in data-structures called elements. All elements in a clique are of the same type. When an element is added to a clique, an event is created, called arrival event.

A clique is created by a KS instantiation and can be shared with other KS instantiations. The kind of interactions allowed between a clique and a KS are dynamically specified via capabilities associated with that clique. Capabilities can be seen as permissions. Two kinds of capability exist: read-only and add-element. Any KS can read from a clique. When a clique has an add-element capability for a $\mathbb{K S}$, this KS may write elements to that clique as well. Modification or deletion of elements is not allowed. The only form of deletion allowed is a form of garbage collection when KSs have signalled to have no more interest in a clique. Summarising, the following operations can be performed on cliques: create, share, copy to (add-element), and copy from (read).

$$
C l=\operatorname{read}-\mathrm{cl} \cdot C l_{l}+\overline{\mathrm{add}-\mathrm{cl}} \cdot C l_{l}
$$

DYNAMIC

\section{A.9.3 Control unit}

AGORA has no control cycle. The architecture is more reminiscent of the original blackboard metaphor than many blackboard architectures 
in the sense that $\mathrm{KS}$ instantiations monitor the blackboard (cliques) for interesting opportunities, specified by activation-patterns. When such an activation-pattern occurs the associated function is executed immediately. The instantiations of KSs can run on different machines, but also on the same machine via time sharing. Load balancing can be changed dynamically, e.g., by KSs, which influences scheduling. However, no selection of tasks is involved. Thus, the description of the life cycle for a $\mathrm{KS}$ is as follows:

$$
L C=\text { c.e. } L C
$$

$D Y N A M I C$

Now we can proceed directly with the description of a complete AGORA system in the following section.

\section{A.9.4 Concurrency}

An AGORA application is described by the following equations. The symbol "|" is used here to denote actual concurrency of the involved. processes. The variable $t$ is used to indicate that the number of several entities in the system, such as KS instantiations and cliques, is dynamic and may change as time $(t)$ progresses.

$$
\begin{aligned}
& A G O R A=\left(K S I_{1}|\ldots| K S I_{n(t)}\left|C \eta_{1}\right| \ldots \mid C I_{m(t)}\right) \backslash L \\
& L=\{\text { read-c1, add-cl }\} \\
& K S I_{j}=\text { match }_{j} . \\
& \left(E-\text { point }_{j_{1}} ; K S I_{j}+\ldots+E \text { point }_{j_{n(j)}} ; K S I_{j}+K S I_{j}\right) \\
& E_{- \text {point }_{k}}=\overline{\text { read-cl }} \cdot E-\text { point }_{k}+\text { add-cl.E-point }_{k} \\
& + \text { create-cl.E-point }+ \text { share-cl.E-point }{ }_{k} \\
& + \text { int-action. E-point } k+\overline{\text { done }} \\
& C l=\operatorname{read}-\mathrm{cI} \cdot C l_{l}+\overline{\mathrm{add}-\mathrm{CI}} \cdot \mathrm{Cl}
\end{aligned}
$$




\section{A.10 GBB}

GBB (Generic BlackBoard development system) is a blackboard framework, which was developed at the University of Massachusetts and which benefitted greatly from the presence of experience with the development of Hearsay-II and DVMT. The developers of GBB (Corkill et al., 1986; Corkill et al., 1987; Gallagher et al., 1988) were mainly concerned with providing efficient blackboard interaction mechanisms. This resulted in a system which seems to be truly generic regarding its blackboard facilities. The opportunities for implementing a control unit and KSs are also generic, but more in the sense of underspecification. However, this does not mean that GBB does not provide facilities to choose from for implementing a control unit and KSs: GBB includes (presently) two complete shells for these aspects. One is a simple control cycle, based on Hearsay-II (Erman et al., 1980); the second is a more elaborate control shell and is derived from BB1 (Hayes-Roth, 1984).

\section{A.10.1 Knowledge sources}

As stated above, most aspects of KSs are left to the user to be specified. This includes for instance (if needed) the condition, KS descriptor, KS instantiations, and binding facilities. Action parts in GBB must be implemented in LISP, although GBB contains a rule-based shell, based on OPS5, as a LISP extension that can be used inside KS action parts.

GBB is equipped with two example shells for control and handling KSs that can be used and tailored for applications. The basic properties of these two shells, called simple shell and $G B B 1$, based on Hearsay-II and BB1 respectively, are described elsewhere (see Sections A.1 and A.8, respectively).

\section{A.10.2 Blackboard}

The emphasis in the design of GBB can be found in the development of mechanisms for defining and using the blackboard. In GBB the blackboard is represented as a tree of spaces. The root and all interior nodes in the tree are called blackboards; the leaves of the tree are called spaces. The spaces contain the actual blackboard objects, called units. 
Spaces resemble levels in other blackboard architectures. Each space has a number of dimensions, each of which can either be ordered or enumerated. A space is uniquely identified by its place in the space tree as specified by the path from root to space (the same space definition. can occur at more than one place in the tree).

The units are the blackboard objects. Units are aggregated data types. Attributes contain: slots, links, dimensional indices, path indices, and paths. The slots contain the actual information of a unit. The place of a unit in a space according to the dimensions of that space is represented by dimensional indices which can be derived directly or indirectly from the values in its slots (a user must specify how). Analogously, the identity of the space (s) to which a unit belongs is represented by path indices and paths which can be derived also directly or indirectly (user-specified) from the slot values. Units can be either named or anonymous. The use of dimensional and path indices greatly increases efficiency of blackboard interaction, by allowing content-based access to units on the blackboard, while minimizing search.

Links are implemented in GBB analogously to slots (and are labelled accordingly). Links must be bidirectional. GBB provides mechanisms for maintaining consistency among the relations represented by links.

$$
\begin{aligned}
B B= & \left\{\text { blackboard }^{+}\right\} \\
\text {blackboard }= & \left\{\text { blackboard }{ }^{*}\right\} \mid\left\{\text { space }^{*}\right\} \\
\text { space }= & \{\text { unit }\} \\
\text { unit }= & \left\{\text { unit-name } \text { opt },(\text { slot, slot-value })^{*},(\text { (ink, unit-name })^{*},\right. \\
& \text { dim-index } \left.^{*}, \text { path-inde } x^{+}, \text {path }^{+}\right\}
\end{aligned}
$$

STATIC

The following interactions can occur with the blackboard: creation and deletion of units; addition to, deletion from spaces, and transportation of units from one space to another; initialisation, access, and updating of slots; and initialisation, access, updating, and removal of links. Each of these interactions results in an event. Per unit type an event-handler must be specified by the user to define when an event is recorded and what information should be sent to the control unit. Exceptions may be specified per unit. Event-handlers can pass on all information related 
with an event without any abstraction.

\section{A.10.3 Control unit}

GBB places no restrictions on mechanisms used for the control unit. While GBB provides two different shells for control and handling KSs (simple shell and $G B B 1$; descriptions of the systems from which these were derived are given elsewhere), which can be used and adapted to specific needs, many other approaches may be implemented by the user. The facilities for specifying and accessing the blackboard also provide a means for recording events, which essentially form the fuel in a blackboard system's problem-solving process. Events are the input to any control unit. It is left to the user to specify in which way events are matched with conditions of KSs, how actions are selected, which information is used for selection of tasks and so on. This flexibility for defining control in GBB makes it pointless to try and describe a life cycle for KSIs.

\section{A.11 Blondie}

Blondie is a blackboard framework, developed in order to become acquainted with important aspects of designing and applying a blackboard architecture. Blondie (Lippolt et al., 1986; Lippolt et al., 1987) shares important aspects with the blackboard architecture of BB1.

\section{A.11.1 Knowledge sources}

Knowledge sources in Blondie consist of a data structure describing the KS, the KS descriptor, and an action part which is a Pop-11 procedure. The KS descriptor specifies in its trigger the blackboard changes, or events, a KS is interested in. The trigger is defined as an abstraction of events (template), without allowing AND or OR combinations. Abstraction is essentially achieved by leaving some attributes of useful events unspecified. When such an event occurs, an instantiation of the KS is created with the triggering event as part of its context. The KS descriptor also contains the name of a procedure, called the precondition, which checks the blackboard for arbitrary complex patterns to 
determine applicability of the KS. The KS descriptor further contains an identification of variables which can be assigned values by the precondition. These values will, together with the triggering event, provide the context for an instantiation of the KS. Finally, a KS descriptor may contain arbitrary, user-definable features in its features attribute that may be relevant for control.

The action part of a KS in Blondie is a noninterruptible Pop-11 procedure, which may incorporate code in several other programming languages (e.g., PROLOG, LISP, and C) and programming paradigms (such as rule-based or frame-based reasoning) in its action parts.

$$
\begin{aligned}
K S & =\{\text { descriptor, action-part }\} \\
\text { descriptor } & =\{K S \text {-name, trigger,precondition, variables, features }\} \\
\text { trigger } & =\text { event-abstraction } \\
\text { precondition } & =\text { Pop-11-code } \\
\text { variables } & =\{\text { variable-name* }\} \\
\text { features } & =\left\{(\text { attribute, value })^{*}\right\} \\
\text { action-part } & =\text { Pop-11-code }
\end{aligned}
$$

When a KS is triggered a data structure is created, containing relevant information about this instantiation of the KS. This data structure is called a knowledge source activation record, or KSAR. This KSAR contains references to the triggering event and the unit that was instrumental in this event, values as assigned to the variables, the same features as the KS descriptor, and a slot for the current priority as assigned by the control unit. A priority can be any number rating the importance of a KSAR against that of other KSARs. Thus, a KSAR may read as follows:

$$
\begin{aligned}
K S A R= & \{K S A R \text {-name, } K S \text {-name, triggering-unit, } \\
& \text { triggering-event, precondition, values, features, } \\
& \text { priority }\}
\end{aligned}
$$




$$
\begin{aligned}
\text { triggering-unit } & =\text { unit-id } \\
\text { triggering-event } & =\text { event } \\
\text { values } & =\left\{(\text { (variable-name, value })^{*}\right\}
\end{aligned}
$$

A KSAR which has been found applicable by its corresponding precondition is called an invocable KSAR, while a not invocable KSAR is simply called triggered.

\section{A.11.2 Blackboard}

The blackboard in Blondie can be subdivided into an arbitrary number of panels, each of which can be subdivided in turn into blackboard levels. Elements on a blackboard level, called units, are collections of attribute-value pairs with an identifier. A panel for control as well as two levels on this panel are predefined. These are the levels to-do-set and chosen-action. They are used by the framework for representing pending and selected activities. Links can be specified via the attributes of units on the blackboard.

Blondie provides procedures for creating and modifying units and for "associative" retrieval of information from the blackboard. The definition of the blackboard is as follows:

$$
\begin{aligned}
B B & =\{\text { control, panel" }\} \\
\text { panel } & =\{\text { panel-id, level" }\} \\
\text { level } & =\{\text { level-id, unit" }\} \\
\text { control } & =\{\text { "control", chosen-action, to-do-set, level" }\} \\
\text { chosen-action } & =\{\text { "chosen-action", unit" }\} \\
\text { to-do-set } & =\{\text { "to-do-set", unit }\} \\
\text { unit } & \left.=\{\text { unit-id, (attribute, value })^{*}\right\}
\end{aligned}
$$

Whenever a unit is created or modified, a data structure, called event, is created to describe this blackboard modification. An event consists of 
attribute value pairs indicating the unit that was part of the modification, the blackboard level and the panel on which the change occurred, the kind of event (creation or modification of a unit), and in case of modification also the name of the attribute that was modified. An event can be defined as follows (each of the items in the describing set are attribute-value pairs):

$$
\begin{aligned}
\text { event } & =\{\text { unit-id,level-id, panel-id, kind, }[\text { attribute }]\} \\
\text { kind } & =\text { "new"|"modified" }
\end{aligned}
$$

STATIC

\section{A.11.3 Control unit}

The control cycle in Blondie is subdivided into three major steps, called Update-to-do-set, Choose-ksar, and Interpret-ksar, respectively, after the corresponding tasks in $\mathrm{BB} 1$. Unlike $\mathrm{BB} 1$, where these tasks were implemented as KSs, Blondie executes its control cycle as a fixed sequence of procedures.

The control cycle starts with Update-to-do-set in which first all events that occurred during the previous cycle are matched against the triggers of all KSs, instantiating those KSs for which a match was found. Then for all triggered (but not yet invocable) KSARs their precondition procedure is run by the procedure test-precondition. This determines the invocable KSARs. Next, for each invocable KSAR a priority is calculated by a user-definable procedure.

In the next procedure, Choose-ksar another user-definable procedure is run to select the most appropriate invocable $\mathrm{KSAR}$ for execution. Once more the precondition is run for the selected KSAR to make sure it is still applicable. The earlier instantiated context for this KSAR can, if necessary, be updated. If the precondition fails, the KSAR becomes a triggered KSAR again and the next best KSAR is selected for execution. Executing the selected KS by the procedure Interpret-ksar completes the cycle. 


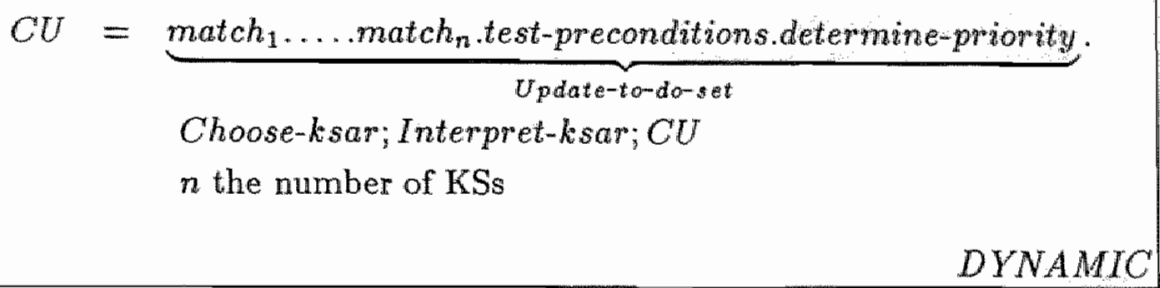

Testing preconditions typically involves searching the blackboard for arbitrary complex patterns of data. Determining priorities of KSARs and selecting a KSAR may involve using data on the blackboard as well as data present in the KSARs, i.e., the descriptions of particular KS instantiations.

As Blondie derives its control from $\mathrm{BB1}$, also the description of the life cycle for a KSI in Blondie equals that in BB1:

$$
\begin{aligned}
L C & =p \cdot L \\
L & =\text { c.s.(e.r. } \overline{\text { done }}+L)
\end{aligned}
$$

$D Y N A M T C$

\section{A.12 Blondie-II}

The parallel blackboard framework Blondie-II was developed as a research tool for intelligent control in robot cells. Blondie-II (Velthuijsen et al., 1987a; Velthuijsen et al., 1987b; Velthuijsen, 1987d; Velthuijsen, $1987 \mathrm{e})$ is an extension of the Blondie system, mainly characterised by its ability to execute KSs concurrently. In Blondie-II three different types of concurrent processes can be recognised: one control unit process, one blackboard process, and an indefinite number of KS executor processes. Concurrency induced several modifications due to the interpretation of KS instantiations as part of the control cycle and to the blackboard operating as a separate process, handling simultaneous requests by concurrent processes. Blondie-II's architecture supports any mapping of processes onto processors. 


\section{A.12.1 Knowledge sources}

Knowledge sources in Blondie-II are like KSs in Blondie, with a few extensions. In Blondie-II the KS descriptor is extended to include references to the (names of) virtual processors capable of executing this KS.

$$
\begin{aligned}
& K S=\{\text { descriptor, action-part }\} \\
& \text { descriptor }=\{\text { KS-name, trigger, precondition, variables } \\
&\text { virtual-processors, features }\} \\
& \text { trigger }= \text { event-abstraction } \\
& \text { precondition }=\text { Pop-11-code } \\
& \text { variables }=\{\text { variable-name }\} \\
& \text { processors }=\{\text { processor-id } \\
& \text { features }=\left\{(\text { attribute, value }\}^{*}\right\} \\
& \text { action-part }=\text { Pop-11-code }
\end{aligned}
$$

KSAR descriptors are extended likewise to include references to processors capable of executing this KS. In Blondie-II a KSAR is described as follows:

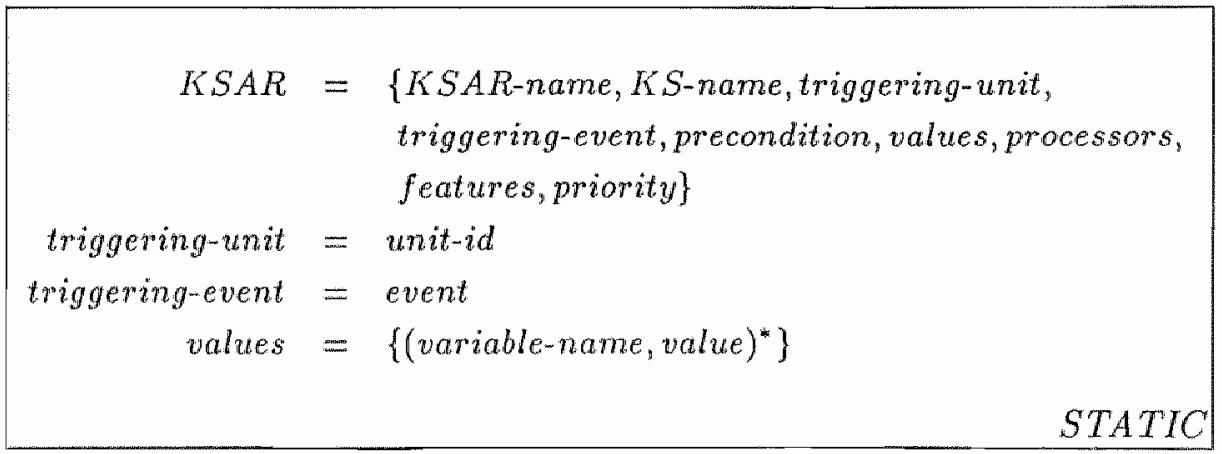

The action parts of KSs are executed by dedicated KS processes. ${ }^{4}$ These KS processes receive commands, specifying the KSAR that has to be ex-

\footnotetext{
"KS processes may be mapped on any number of processors: collection of IKS processes may be executed on one processor or on different processors. This fact has no direct influence on the architecture and is thus left out of this description. See for more details the description of Blondie-II in Chapter 4.
} 
ecuted, execute that $\mathrm{KSAR}$, and announce their availability upon completion of the task. This leads to the following definition for KS processes ( $j$ ranges over the $m \mathrm{KS}$ processes):

$$
K S_{j}=\overline{\text { commandj }_{j}} \cdot \text { Exec-task }_{j} ; \text { ready }_{j} \cdot K S_{j}
$$

DYNAMIC

The process Exec-task coincides with the execution of the action part of some $\mathrm{KS}$ with a context as specified by the KSAR representing this particular task. Typically, an action part will interact with the blackboard. Before engaging in an interaction an action part may wish to lock a region of the blackboard in order to secure exclusive access to specific data. KSs only communicate with the blackboard.

$$
\begin{aligned}
& E_{x e c-t a s k_{j}}=\text { read-lock }_{j} \cdot \text { Exec-task }_{j}+\text { read-unlock } j \cdot \text { Exec-task }_{j}+
\end{aligned}
$$

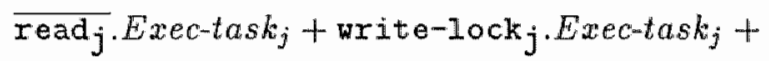

$$
\begin{aligned}
& \text { write-unlock }_{j} \text {. Exec-task } \text { Exite }_{j} \text {. Exec-task } \text { Er }_{j}+ \\
& \text { internal-activity. Exec-task }{ }_{j}+\overline{\text { done }}
\end{aligned}
$$

DYNAMIC

\section{A.12.2 Blackboard}

While the blackboard in Blondie-II can be partitioned in the same way as in the Blondie framework, it is implemented as a separate process, handling requests from $\mathrm{KSs}$ and the control unit. Concurrent requests are sequentialised (by the operating system) and handled in some order according to priority (requests from the control unit have a higher priority than requests from KS processes). As in the Blondie framework the partitioning of the blackboard is described by the following:

$$
\begin{aligned}
B B & =\{\text { control }, \text { panel } \\
\text { panel } & =\left\{\text { panel-id, } \text { level }^{*}\right\}
\end{aligned}
$$

(continued on next page)

STATIC 


$$
\begin{aligned}
\text { level } & =\{\text { level-id, unit" }\} \\
\text { control } & =\{\text { "control", chosen-action, to-do-set, level" }\} \\
\text { chosen-action } & =\{\text { "chosen-action", unit }\} \\
\text { to-do-set } & =\{\text { "to-do-set", unit" }\} \\
\text { unit } & =\{\text { unit-id, (attribute, value })\}
\end{aligned}
$$

Events are generated, as in Blondie, each time a unit is created or modifyed on the blackboard. Additionally, in Blondie-II an event is generated for each deletion of a unit.

$$
\begin{aligned}
\text { event } & =\{\text { unit-id, level-id,panel-id, kind, }[\text { attribute }]\} \\
\text { kind } & =\text { "new"|"modified"|"deleted" }
\end{aligned}
$$

STATIC

In addition to creation, modification, and deletion of units on the blackboard, the blackboard process allows locking of regions (levels or panels) of the blackboard by $K S$ processes. A read-lock for a specific region by a KS process prevents other KS processes from changing the data in that region until the lock is released. A write-lock also prevents other $\mathrm{KS}$ processes from reading write-locked data.

This mechanism was introduced to enable application writers to ensure reliability and consistency of data on the blackboard at all times. As Blondie-II was developed for robot control and the blackboard state also represents the current world state in which effectors are moving, incorrect data on the blackboard might result in damaging collisions.

There are no explicit mechanisms provided by Blondie-II for avoiding deadlock caused by concurrent processes holding locks, but adhering to the simple (although possibly time-consuming) strategy of unlocking all held locks before requesting for additional locks achieves this.

The blackboard process can now be described ( $j=0$ represents communication with the control unit; $j=1, \ldots, m$ with the KS processes): 


$$
\begin{aligned}
& B B=\sum_{j=0}^{m}\left(\overline{\text { read-Iock }} \cdot B B+\overline{\text { read-unlock }} \cdot B B+\text { read }_{j} \cdot B B+\right. \\
& \left.\overline{\text { write-lock }} \cdot B B+\overline{\text { wite-unlock }} \cdot B B+\overline{\text { write }_{\mathrm{j}}} \cdot B B\right)
\end{aligned}
$$

DYNAMIC

Actually the blackboard process is more complex, as the mechanism of locking is more restrictive in allowed sequences of interaction than is represented here, but inclusion of this needs specification of regions as parameters for the various actions. This is not naturally included in our formalism.

\section{A.12.3 Control unit}

The control cycle of Blondie was modified in such a way for Blondie-II that the execution of KSs is handed over to KS processes.

$$
\begin{aligned}
& C U=\sum_{j=1}^{m}\left(\text { ready }_{j} \cdot \text { match }_{1} \ldots \text { match }_{n}\right. \text {.test-preconditions. } \\
& \text { determine-priority.choose-ksar } \left.{ }_{j} \text {.command } j . C U\right) \\
& m \text { the number of KS processes, } n \text { the number of } K S s
\end{aligned}
$$

In each cycle all not yet processed events are matched against the triggers of all KSs including those that can not be executed by the KS process that has announced itself to be available. Then among the invocable KSs the best one, which also can be run by the KS process under consideration, is selected (action choose-ksar). Selection of KSARs can use information available on the blackboard as well as data represented in the KSARs. The features-slot in the ISS descriptor may contain information describing resources that may be needed by a KS (such as the locks) or indicating the suitability of a specific KS process for executing this particular KS.

The life cycle for KSIs in Blondie-II equals that for Blondie and BB1: 


$$
\begin{aligned}
L C & =p \cdot L \\
L & =c . s \cdot(\text { e.r. } \overline{\text { done }}+L)
\end{aligned}
$$

DYNAMIC

\section{A.12.4 Concurrency}

The dynamic definition of Blondie-II is now defined by the following (the symbol "|" indicates in this case actual opportunities for distribution):

$$
\begin{aligned}
& B B S=\left(C U|B B| K S_{1}|\ldots| K S_{m}\right) \backslash L \\
& C U=\sum_{j=1}^{m}\left(\overline{\text { ready }_{j}} \cdot \text { match }_{1} \ldots \text { match }_{n} \cdot\right. \text { test-preconditions. } \\
& \text { determine-priority.choose-ksar } \text {. command }_{\mathrm{j}} . C U \text { ) } \\
& m \text { is the number of KS processes, } n \text { the number of KSs } \\
& \begin{aligned}
B B= & \sum_{j=0}^{m}\left(\overline{\text { read-lock }} \cdot B B+\overline{\text { read-unlock }} \cdot B B+\operatorname{read}_{\mathbf{j}} \cdot B B+\right. \\
& \left.\overline{\text { write-lock }} \cdot B B+\overline{\text { write-unlock }} \cdot B B+\overline{\text { write }_{j}} \cdot B B\right)
\end{aligned} \\
& K S_{j}=\overline{\text { command }_{j}} \cdot \text { Exec-task }_{j} ; \text { ready }_{j} \cdot K S_{j} \\
& L=\cup_{j=1}^{m}\left\{\text { read }_{j}, \text { command }_{j}, \text { read-lock }_{j}, \text { read-unlock }_{j}, \text { read }_{j},\right. \\
& \text { write-lock } \left.k_{j} \text {, write-unlock }{ }_{j}, \text { write }_{j}\right\}
\end{aligned}
$$

DYNAMIC

\section{A.13 Blondie-III}

Blondie-III was developed as a research tool for studying Distributed Artificial Intelligence. Blondie-III (Florescu et al., 1988a) is an extension of the blackboard framework Blondie. Blondie-III is a distributed blackboard shell, i.e., it provides an environment in which several complete Blondie blackboard systems can cooperate, resulting in a distributed problem-solving network, where the nodes are the blackboard systems. Apart from some small optimalisations Blondie-III extends 
Blondie mainly by its mechanisms for communication between nodes (blackboard systems) in the system.

\section{A.13.1 Knowledge sources}

The KSs in Blondie-III have the same form and specification as KSs in Blondie. There is one notable exception which lies in the allowed complexity of the trigger. In Blondie-III combinations of events (apart from the usual abstractions of events) can be specified for triggering KSs. Combination functions are AND, OR, and FOLLOWED-BY, allowing arbitrary complex patterns of events, which may occur independently during any of the cycles of the control unit. The definition of $\mathrm{KS}$ S reads:

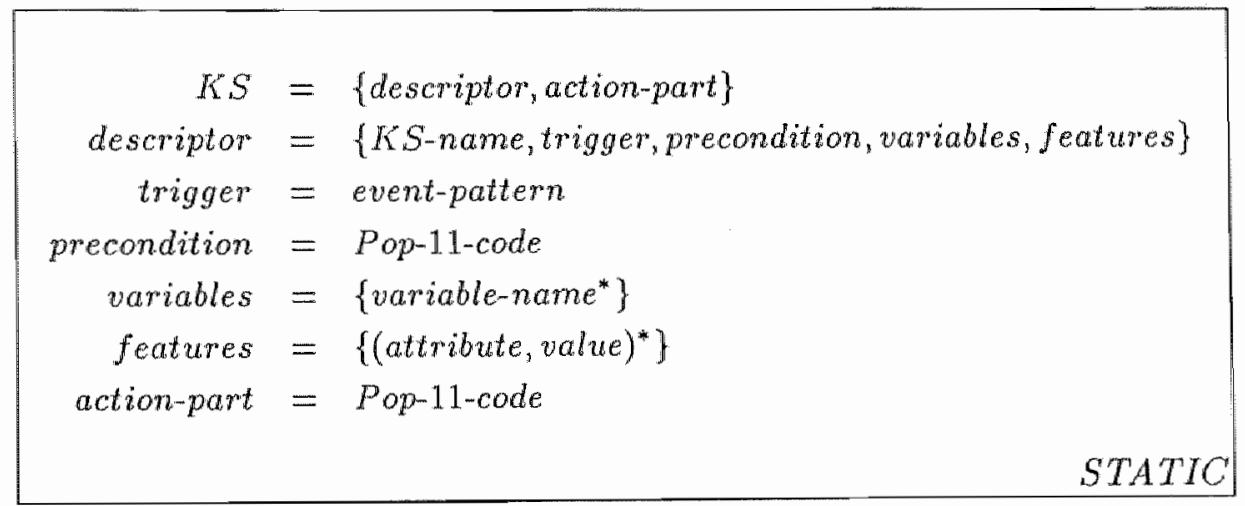

The definition of a KSAR is left unchanged:

$$
\begin{aligned}
K S A R= & \{K S A R-n a m e, K S-n a m e, \text { triggering-unit, } \\
& \text { triggering-event, precondition, values, } \\
& \text { features, priority }\} \\
\text { triggering-unit }= & \text { unit-id } \\
\text { triggering-event }= & \text { event } \\
\text { values }= & \left\{(\text { (variable-name, value })^{*}\right\}
\end{aligned}
$$

Apart from communicating with the blackboard the action part of a KS may engage in some communication with other nodes in the problemsolving network. Incoming communication can only be treated directly 


$$
\begin{array}{ll} 
& \frac{\text { request-event.write.Handle-comm }+}{\text { stop-request-event.write.Handle-comm }+} \\
& \text { inform.Handle-comm + done } \\
\text { Interpret-ksar = } & \text { KS-action }
\end{array}
$$

A request for notification of events is stored by the receiving node as well and will be acted upon when relevant events occur (represented by the last but one term in the definition of Handle-comm).

The description of the life cycle for a KSI in Blondie-III is slightly different from those in Blondie and Blondie-II. The control cycle in Blondie-III includes the same extra test of the condition upon selection of a KSI, but the interpretation of a negative result may vary. In case the extra test was negative, a KSI may either be returned to the ranks of present KSIs or be completely removed from the system:

$$
\begin{aligned}
L C & =p \cdot L \\
L & =c \cdot s \cdot(e \cdot r \cdot \overline{\text { done }}+L+\overline{\text { done }})
\end{aligned}
$$

\section{A.13.4 Concurrency}

The behaviour of one node is represented by the following set of definitions ( $n$ the number of KSs):

$$
\begin{aligned}
\text { NODE }= & (C U \mid B B) \backslash L^{\prime} \\
L^{\prime}= & \{\text { read, write }\} \\
C U= & \text { Handle-comm; } \\
& \text { match }_{1} \ldots \text { match }_{n} . \text { test-preconditions. } \\
& \text { determine-priority.choose-ksar.Interpret-ksar; } C U
\end{aligned}
$$




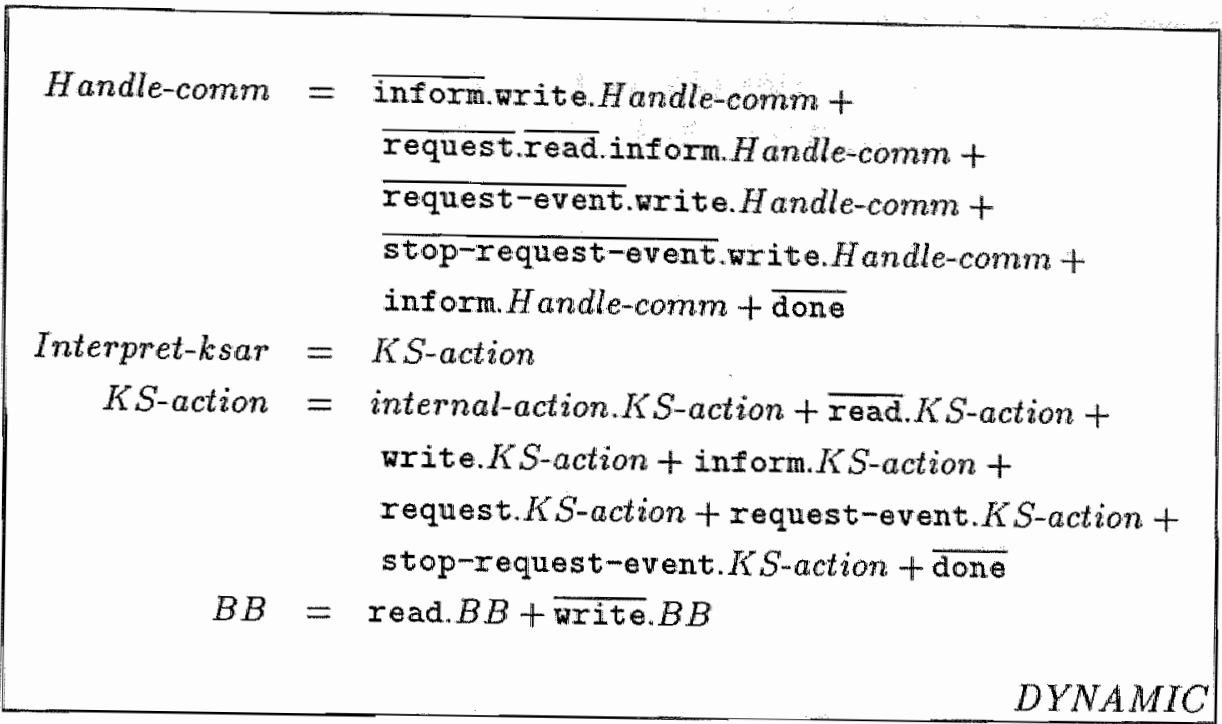

A problem-solving network consists of a number of nodes, leading to the following definition ( $r$ the number of nodes):

$$
\begin{aligned}
\text { NETWORK }= & \left(\text { NODE }|\ldots| N O D E_{r}\right) \backslash L \\
L= & \{\text { inform, request, request-event }, \\
& \text { stop-request-event }\}
\end{aligned}
$$

DYNAMIC

\section{A.14 CASSANDRA}

The CASSANDRA architecture provides a framework concentrating on modularity. Craig (1989) presents the architecture of CASSANDRA as a blackboard-like architecture where levels are seen separately, each with its own collection of KSs and own (local.) control. Information exchange between different levels is handled via message passing among so called Level Managers, the components supervising the process associated with one level. Actually, the CASSANDRA framework is a special instance of the distributed blackboard framework, but with a notable restriction to only one level for the blackboard in each node of the distributed blackboard architecture. 
While the design of the CASSANDRA architecture concentrates on the modularity of the framework, many other options within the architecture (such as the definition of $\mathrm{KS}$ conditions and condition matching) are left unspecified.

Two applications of the CASSANDRA architecture are described in (Craig, 1989): CASSANDRA-II for monitoring aircraft movements in controlled airspace; and WordSys for word recognition according to the model as described by McClelland and Rumelhart (1981; 1982). These two applications illustrate some of the allowed generality.

The representation given in this section is concerned with the CASSANDRA architecture itself, not the applications. The Level Managers are described as nodes in a problem-solving network. Since 1989, Craig has created an updated design for CASSANDRA, which can be found in (Craig, 1991). As there is no information currently available about an actual implementation, we base our description entirely on (Craig, 1989).

\section{A.14.1 Knowledge sources}

Each Level Manager (LM) has its own collection of KSs. A KS in CASSANDRA is a relatively simple condition-action pair. The action part of a KS in CASSANDRA is a set of rules. In the application CASSANDRA-II these rules are executed sequentially. Action parts of KSs can modify the local information on the blackboard within its own LM. Action parts may send messages to other LMs as well, which may decide to place the contents of received messages on their own local blackboard. Similarly, KSs can read information from the local blackboard and send requests for specific data to other LMs. This data can not be received by the KSs directly, as the message passing communication method in CASSANDRA is asynchronous. CASSANDRA uses dedicated $\mathrm{KSs}$ for deleting entries from a level.

The dynamic description of a KS action part for a KS within a certain LM is now as follows $(i, j=1, \ldots, m$, $m$ the number of LMs in an application): 


$$
\begin{aligned}
& K S \text {-action }_{i}=\overline{\text { read }}_{i} \cdot K S \text {-action }+ \text { write } \cdot K S \text {-action } i \\
& \text { message }_{j} \times S \text {-action }_{i}+\text { int-action. } K S \text {-action }+ \\
& \overline{\text { done }}
\end{aligned}
$$

CASSANDRA provides for a condition definition which uses both information about blackboard state and events. The part of the (event-based) condition is called trigger; the state-based part is called precondition. The trigger can only match events generated within the same LM. The precondition can match also values present in other LMs. As verification of values in another LM may involve an indefinite number of time, a three-valued logic is used for evaluating preconditions (the third logical value undefined being reserved for predicates not yet resolved by other LMs). The allowed complexity of both trigger and precondition can be redefined for each application.

KS instantiations record only the entry in the local blackboard that triggered this particular instantiation together with an identification of the KS. There are no mechanisms provided for binding variables.

\section{A.14.2 Blackboard}

In CASSANDRA each blackboard level is a separate entity. The levels may occur in any hierarchy. Each level has a Level Manager (LM) associated with it. The LM acts as a local control unit and provides communication facilities with other LMs. The units on a level are called entries. Within one LM the entries can be all of one type or of a set of types (this actually extends the usual notion of a level, where all units would have the same type; a level in CASSANDRA is in this respect similar to a complete blackboard). The contents of a level in CASSANDRA can only be inspected or modified directly by KSs within the same LM. Communication via the LMs can provide indirect read and write actions by KSs within other LMs. Deletion of entries can only be achieved by dedicated KSs. When an entry is deleted, pending actions related to this 


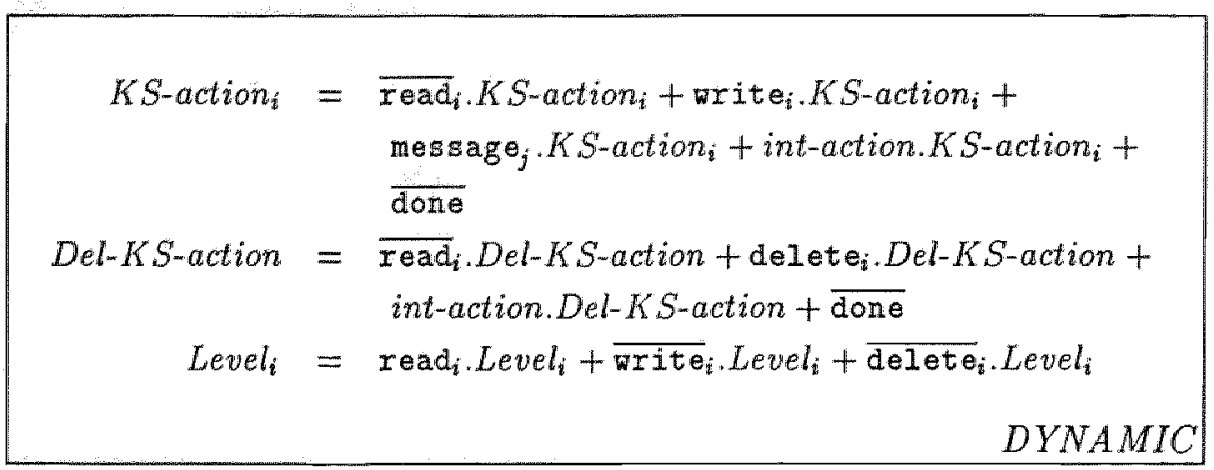




\section{Index}

Pagenumbers in italics refer to formal definitions of the corresponding items.

ABLS, 42,43

ABSTRIPS, 87

ACCORD, 53

ACP, 149

Address Block Localisation

System, see ABLS

ADL, 33, 34, 52, 54, 130

ADP, 48,49

AGE, 20,43, 52, 172, 184, 189,

232,316

AGORA, 178, 184, 190, 991

anchor, 13

architecture, 6

Ariadne-I, 52, 53, 207

Assumption-based Truth

Maintenance System, 13, 206

ATMS, see Assumption-based

Truth Maintenance System

ATOME, 47, 52, 54

Backus-Naur Form, see BNF

Basar, 29, 30, 50, 105

basic contral loop, see control loop

$\mathrm{BB}^{*}, 53$

BB-SPACE, 47, 48

BB $1,19-21,45,52,53,57,72,161$,

$170,184,190,233,253,326$

$\mathrm{BBC}, 52,54$

BBF, 47,48

BEDS, 47,48 blackboard, $1,12,59,92,168,187$, 203

data consistency, $31,91,102$, $104,194,210$

interaction, $62,150,169,172$, 193,208

level, $15,59,168,187$

link, 16, 137, 169, 174, 193, 207

asymmetric, 169

bidirectional, 137

labelled, 169

symmetric, 169

unidirectional, 137

locking, 32, 102

object hierarchy, 169, 187

organisation, 187, 206

panel, $15,59,168,187$

purpose, 203

region, 102

sequentialised access, 31,91 , 102

state, 157

state change, 157,171

tagging, 32,102

transactions, $30,32,102$

unit, $60,168,193$

blackboard application, 6

blackboard architecture, 6

blackboard framework, 6,51

blackboard generator, 51,148

blackboard metaphor, $1,89,104$, 
150,159

blackbaard methodology, 265

blackboard model, 6

blackboard model of planning, 39

blackboard model of reacling, 36

blackboard shell, 6

blackboard system, 6,155

Blondie, $8,20,52,54,57,183$,

$191,256,397$

Blondie-II, 8, 29, 30, 47, 48, 52, $54,83,183,191,341$

Blondiem III, $8,34,35,46,47,52$,

$54,109,183,191,3 / 6$

$\mathrm{BNF}, 150$

bottom-up processing, 24, 232

$\mathrm{BSG}_{1} 47,48,52,54,148$

CAGE, 29, 30, 52, 53, 104, 184 189,319

CAIBL, 33,34

Calculus of Communicating

Systems, see CCS

CASSANDRA, 33, 34, 47, 52, 53, $130,179,184,191,351$

CASSANDRA-II, 48

causality, 149, 181

CCS, $149,179,181$

circumscription, 174

class bierarchy, 15

CODGER, $29,30,47,48,105$

cognitive model, 4, 5, 35

cognitive science approach, 4

Communicating Sequential

Processes, see CSP

communication, $16,85,94,110$,

113,123

asynchronous, $17,203,226$

blackboard method, 18

broadcasting, 203

data sharing, 110

message passing, 94, 112, 123

party-line method, 17

primitives, 123

private-line method, 17 reserved-spot method, 18

singlecasting, 204

synchronous, 17

task sharing, 110

unidirectional, 204

computer-system architecture, 6

Conceptual Supervision Model, see CSM

concurrency, 28, 83, 109, 164, 199,

208,220

asynchronous, 104

synchronous, $104,165,221$

condition, see knowledge source condition part

consumption, 209

context, 155

Contract Net Protocol, 125

control, 22, 198, 219

agenda, 205

coherence, 179

convergence, 180

data-driven, 25, 87, 229

event-driven, 25,87

flow of control, 215

focus, 233,264

goal-directed, $25,87,229$

inertia, 245,248

opportumistic, 24

pulase, 264

policy, 204, 264

strategy, 204, 264

control cycle, 159,198

control loop, $23,72,98,115,161$

control unit, $2,22,72,90,92,98$, 156,198

distributed control, 164

parallel, 164

COPS, 33, 34, 130

Crysalis, 15,46

CSM, 33, 34

CSP, 149

DAI, see Distributed Artificial Intelligence 
deadlock, 103, 161

Designer Fabricator Interpreter, see DFI

DESTINY, 50

DFI, 49, 50

DISPROS, $33,34,47,48$

Distributed Artificial Intelligence, $33,109,123$

distributed blackboard architecture, 3, 28, 32, 90, 110, 143, 164, 199

Distributed Problem Solving Kernel, see DPSK distributed problem-solving, 109 distributed problem-solving network, 33, 109

Distributed Vehicle Monitoring Testbed, see DVMT

DOLPHIN, 42,43

DPSK, 30, 31, 105

DPSN, see distributed problem-solving network

DSAM, 42,43

DVMT, $33,34,44,129,184,188$, 234,308

Edinburgh Prolog Blackboard Shell, 52, 54

engineering approach, 4

Erasmus, 34, 35, 45, 52, 54, 130, 148

event, $19,64,79,120,172,194$, 211,216

absence of event, 211

clock event, 194

combinations, $79,120,173$,

196, 217

problem, 194

event structures, 149

formalisation, 147

fuzzy sets, 208

GBB, $14,15,44-46,52,53,172$, $174,184,185,190,395$
GEST, 52, 54, 105

goal, $25,194,203,205,229$

relationships, 230

grammar system, 31,180

GUARDIAN, 44,45

HASP, see HASP/SLAP

HASP/SIAP, 15, 19, 23, 27, 44, $80,184,189,319$

HCVM, 29, 30, 47, 48, 105

HCVM-2, 29, 30

Hearsay-I, 36

Hearsay-II, 1, 15, 18-20, 22, 23, $27,29,30,36,42,50,103$, $184,188,232,234,900$

Hearsay-III, 15, 20, 52, 79, 80, 184, 188,904

Heuristic Control Virtual Machine, see HCVM

history, 179

HITS, 48, 49

HOPES, 34, 35, 44, 105

hypotheses, 91

hypothesis, $1,12,13,36,193,226$

hypothesize-and-test paradigm, 36 , 226

immediate action, 218

island, 13

KIM, 30, 31, 49, 50, 104

knowledge source, $1,19,65,170$, 195,211

action part, 19, 170, 196, 213

applicability, 162

condition part, 19, 162, 170,

$171,196,212$

descriptor, $66,98,170,195$

execution, 169,220

initiator, 211,216

instantiation, $20,66,68,155$,

197,254

life cycle, $17 \%, 218$

selection, 162,200, 220,230 
variable binding, $67,170,195$, $214,254,255$

knowledge source activation record, $21,66,68,170$

inwocable, 68

triggered, 68

Knowledge-Based Integration

Manager, see KIM

KS, see knowledge source

KS cycle, 90, 152, 198

KSAR, see knowledge source activation record

KSI, see knowledge source instantiation

latency, 226

livelock, 161

LUPINO, 45,46

machine learning, $112,222,227$, 268

Mirage, 29, 30

MXA, 44, 52, 53, 232

NBB, 53

negotiation, 125

neural networks, 37

non-blackboard reimplementation, $51,81,227$

nonmonotonic reasoning, 194, 209

object-oriented programming, 15, $18,105,223$

OFMSPERT, 44,45

OPM, 15, 26, 27, 40, 45, 46

opportunistic problem solving, 24

OPUS, 52, 54

parallel blackboard architecture, 3, $28,29,83,164,199$

Parallel Distributed Processing, 37 parallelism

data parallelism, 29

pipeline parallelism, 29

Petri-Nets, 149
Pilot's Associate, 29, 30, 44, 45, 105

planning, 26

POLIGON, 15, 52, 53, 79, 178 , $184,189,929$

precondition, 66, 72, 171, 174, 196, 216

PROBE, 46, 52, 54

problem solving, 5

problem-solving state, 5

problem space, 5

process algebra, see $\mathrm{ACP}$

PROTEAN, 13, 27, 46, 53

PSEIKI, 42, 43

PUFF, 42, 43

PVIM $_{4} 44,45$

RARE, 49, 50

RC2, 42, 43

robot cell, 83 control, 83, 106

rule-based programming, 141, 223, 232

SARGE, 46

scheduler, 22, 154

SIAP, see HASP/SIAP

SIGHTPLAN, 53

situated action, 28, 106, 207

spreading activation network, 230 , 238

stop criterion, $73,118,161,163$, 201

STRIPS, 87

$\mathrm{SU} / \mathrm{P}, 15,46$

$\mathrm{SU} / \mathrm{X}, 44$

TABULA, 46

TEAM-CPS, 33,34

termination, see stop criterion

TMS, see Truth Maintenance System

top-down processing, 24, 232

transactional blackboards, see

blackboard transactions 
TRICERO $, 42,43$

trigger, $66,72,171,179,196,216$

Truth Maintenance System, 13, 206

Umass Schema System, 42

virtual blackboard model, 32

$\mathrm{Z}, 149,179$ 


\section{Summary}

\section{The Nature and Applicability of the Blackboard Architecture}

The blackboard architecture is a computer-program architecture for knowledge-based systems. This architecture can be illustrated best by the metaphor that lent it its name. Imagine several specialists with different expertise required to solve a problem cooperatively. The specialists are gathered around a blackboard for communicating intermediate results and hypotheses. As soon as data has been written on the blackboard by one of the specialists, other specialists may decide that they can use that information to contribute to the problem-solving process. These specialists in turn write their own findings on the blackboard, thus providing possibly interesting information for other specialists to continue the problem-solving process. The process so continues until either a solution to the problem is found or no specialist is able to proceed on any of the data present on the blackboard.

The blackboard architecture models this type of problem-solving behaviour. Separate, otherwise independent computer programs, called knowledge sources, are given the role of specialists. A global data structure (the blackboand) is used to write and read intermediate results and hypotheses. Knowledge sources interact only via the blackboard. In many blackboard systems (a computer program with a blackboard architecture) a control unit is added. The control unit selects the most suitable knowledge source when various knowledge sources can contribute at the same time. Metaphorically, a control unit is given the role of discussion leader. 
In practice it appears that many choices have to be made during the design and implementation of a blackboard system. Different choices imply different characteristics of the resulting system. This thesis investigates the range of possible options, the relations between options and characteristics, and the consequences of choosing a certain option for the applicability of the blackboard architecture. The following presents abstracts of the chapters in this thesis.

Chapter 1 presents the blackboard architecture, defines terminology used in this thesis, describes the problem definition, and provides an overview of the contents of the chapters.

Chapter 2 provides background information on the blackboard architecture in general. The sections discuss subsequently the blackboard, the knowledge sources, and the control unit in a blackboard system. These discussions address the purpose of each component and some of the more important variations. Next, some examples of concurrent blackboard systems are described. Chapter 2 also contains descriptions of two cognitive models based on the blackboard architecture. The chapter concludes with an overview of existing applications of the blackboard architecture.

We have designed and implemented a number of blackboand frameworks which are presented in the Chapters 3,4 , and 5. A blackboard framework is a computer program with a blackboard architecture, but without application-specific elements. A blackboard framework provides program elements necessary for implementing a blackboard system, without having to implement these elements for each application again. The first of these frameworks is called Blondie and is based on BB1. Blondie was developed mainly as an instrument to gain practical experience with the blackboard architecture. Analogously, the description of Blondie in Chapter 3 is meant as an illustration of how a blackboard system can be built and how a blackboard system operates.

The second version of Blondie, called Blondie-II, is described in Chapter 4. Blondie-II was developed for application in robot control. This required concurrent execution of knowledge sources. Blondie-II supports this. We call such an architecture a parallel blackboard architecture. Chapter 4 also discusses in what way the intended application influenced our design decisions.

Our third blackboard framework, Blondie-III, is described in Chapter 5. 
Blondie-III was developed as a tool for studying the feasibility of using Distributed Artificial Intelligence techniques for a telecommunicationnetwork configuration problem. Blondie-III is a distributed blackboard framework, designed for implementing a number of cooperating blackboard systems. Blondie-III has been applied on a telecommunicationnetwork configuration problem, also described in Chapter 5. The distribution of the problem-solving task over blackboard systems coincides with functional and geographical distributions within the application domain.

After gaining experience with implementing and applying blackboard frameworks, we wanted to acquire a better insight in the nature and applicability of the blackboard architecture. For this we developed a formalisation for describing blackboard architectures precisely and consistently. Chapter 6 contains this formalisation.

Subsequently, the formalisation was used to describe fourteen existing blackboard architectures. These descriptions are collected in Appendix A. These fourteen architectures include Blondie, Blondie-II, and Blondie-III. Furthermore, eleven architectures are described which are well-known from the literature. Since publications about these eleven architectures are readily available, these architectures serve as examples regularly. Hence, the collection of design and implementation options selected within these architectures provide a large subset of options within all blackboard architectures.

The formal descriptions of architectures in Appendix A were used for an analysis. This analysis was directed at finding answers to the following questions: which options exist and have been used in blackboard systems; which additional and meaningful options are conceivalble; what is the relationship between selected options and characteristics of the resulting architecture; in what way can the blackboard architecture be distinguished from other, comparable program architectures. Answers to these questions are presented in Chapter 7 . In fact, these answers form the core of this thesis as they try to define the nature of the black. board architecture.

A significant problem typically encountered during the development of a blackboard system is the need for an optimal strategy for selecting knowledge sources for execution: how can a knowledge source be se. lected which seems to be the most efficient and effective in light of cur- 
rent information. Blackboard systems are essentially 'data driven': a knowledge source may be selected when data arrives on the blackboard which signifies that the knowledge source can contribute. It is desirable, however, to evaluate the desirability of executing a knowledge source by examining how well the knowledge source is expected to contribute to the goals of the system. Such 'goal-directed' selection needs to be integrated with the more natural, data-driven method of knowledge source selection. Although several approaches exist towards incorporating goaldirected selection in blackboard systems, we do not know of approaches that realise a true integration of data-driven and goal-directed selection, as is illustrated in Chapter 8 . We propose a method, based on a dynamical network, to provide true integration. This method is presented in Chapter 8 . The method has yet to be verified in a real-world application domain.

General conclusions to the work presented in this thesis can be found in Chapter 9. More detailed conclusions are provided by the evaluations at the end of most chapters. Chapter 9 also discusses future research. This discussion focuses on research aiming at the development of a blackboard methodology. Such a methodology should support the design and implementation process and help making the right decisions during the development of a blackboard system for a particular application domain. 


\section{Samenvatting}

\section{De Essentie en Toepasbaarheid van de Blackboard-Architectuur}

De blackboard-architectuur is een programma-architectuur voor kennissystemen. Deze architectuur dankt zijn naam aan de volgende metafoor. Men kan zich een situatie voorstellen waarin enkele personen geacht worden gezamenlijk een probleem op te lossen. Deze personen verblijven in een kamer, waar een schoolbord is opgehangen. Dit schoolbord ("blackboard" in het Engels) wordt gebruikt door de personen om met elkaar te communiceren en wel op de volgende wijze. Een beschrijving van het op te lossen probleem wordt op het schoolbord geschreven. We gaan er nu van uit dat tenminste één van de personen een aanknopingspunt ziet in deze probleembeschrijving tot het leveren van een bijdrage aan het oplossingsproces. Dit levert een tussenresultaat of een mogelijke hypothese op die ook op het schoolbord wordt geschreven. Een aldus verschenen tussenresultaat of hypothese kan op zijn beurt weer aanknopingspunten bieden voor nieuwe bijdragen van de aanwezigen. Door het steeds reageren door personen op tussenresultaten verkregen door anderen ontstaat een oplossings- proces dat pas stopt wanneer niemand meer een bijdrage kan leveren of wanneer een oplossing gevonden is.

Een blackboard-architectuur kopieer deze methode van probleemoplossen in een computerprogramma. In een dergelijk programma wordt het schoolbord geimplementeerd door middel van een globale datastructuur, het blackboard geheten. De samenwerkende personen komen overeen met (deel)programma's die uitsluitend met elkaar interacteren door het schrijven en lezen van informatie in de globale datastructuur. De deelprogramma's in een blackboard-architectuur heten kennisbronnen. Vaak 
wordt aan een computerprogramma met een blackboard-architectuur (een blackboard-systeem) nog een component toegevoegd dat tot doel heeft de meest geschikte kennisbron te kiezen indien verschillende kennisbronnen tegelijkertijd een bijdrage kunnen leveren. Een dergelijke component noemen we een beslissingseenheid en kan binnen de metafoor worden opgevat als een discussieleider.

Het blijkt in de praktijk dat er bij het ontwerpen en implementeren van een blackboard-systeem veel verschillende keuzes gemaakt kunnen worden. Daarbij leiden verschillende keuzes tot verschillende eigenschappen van het resulterende programma. Dit proefschrift onderzoekt welke variaties mogelijk zijn, welke relaties bestaan tussen gekozen varianten en eigenschappen van het resulterende programma en wat de consequenties zijn van dergelijke keuzes voor de toepasbaarheid van de blackboardarchitectuwr. In het nu volgende worden de hoofdstukken in het proefschrift samengevat.

Hoofdstuk 1 beschrijft kort de blackboarcl-architectuur, presenteert definities van termen die veel worden gebruikt binnen het proefschrift, geeft de probleemstelling voor het proefschrift aan en geeft een overzicht van wat in de hoofdstukken beschreven wordt.

Hoofdstuk 2 verschaft algemene achtergrond informatie over de blackboard-architectuur. Achtereenvolgens worden het blackboard, de kennisbronnen en de beslissingseenheid binnen een blackboard-systeem besproken. Hierbij komen de functie van iedere component en een aantal van de belangrijkste verschijningsvormen aan bod. Vervolgens worden enkele voorbeelden van parallellisme in blackboard-architecturen beschreven. Hoofdstuk 2 bevat verder nog beschrijvingen van een tweetal cognitieve modellen gebaseerd op de blackboard-architectuur. Hooflstuk 2 besluit met een overzicht van bestaande toepassingen van de blackboardarchitectuur.

Hoofdstukken 3,4 en 5 beschrijven achtereenvolgens verschillende versies van een blackboard-shell door ons ontwikkeld. Een blackboard-shell is een computerprogramma met een blackboard-architectuur, maar zonder toepassings-specifieke programmatuur. Een blackboard-shell biedt de programma-elementen die noodzakelijk zijn bij het implementeren van een blackboard-systeem, zodat deze elementen niet voor iedere toepassing opnieuw geimplementeerd hoeven te worden. De eerste versie van onze blackboard-shell heet B]ondie en is voornamelijk gebaseerd op de 
blackboard-shell BB1. Blondie is ontwikkeld teneinde ervaring op te doen met het concept van de blackboard-architectuur. De beschrijving van Blondie in Hoofdstuk 3 is ook hoofdzakelijk bedoeld als concreet voorbeeld van hoe een blackboard-systeem kan worden gerealiseerd en hoe een dergelijk systeem werkt.

De tweede versie van Blondie, Blondie-II genaamd, wordt beschreven in Hoofdstuk 4. Blondie-II is ontwikkeld ten behoeve wan toepassing in robot besturing. Daartoe moest het mogelijk gemaakt worden om kennisbronnen gelijktijdig uit te voeren. Blondie-II ondersteunt parallelle uitvoering van kernisbronnen. Een dergelijke architectuur heet een parallelle blackboard-architectuur. Verder wordt beschreven hoe het toepassingsdomein robot besturing van invloed was op de ontwerpbeslissingen.

In Hoofdstuk 5 wordt Blondie-III beschreven. Blondie-III is ontwikkeld als hulpmiddel bij onderzoek naar de haalbaarheid van het gebruik van gedistribueerde kunstmatige intelligentie technieken in een telecommunicatie-netwerkconfiguratie probleem. Blondie-III ondersteunt de implementatie van verschillende met elkaar samenwerkende blackboardsystemen. Een dergelijke architectuur wordt een gedistribueerde blackboard-architectuur genoemd. Hoofdstuk 5 beschrijft tevens een prototype systeem voor telecommunicatie-netwerkconfiguratie, ontwikkeld met behulp van Blondie-III. In dit prototype werken vijf blackboardsystemen samen met als doel een nieuwe verbinding in een telecommunicatie-netwerk te configureren. De opdeling wan het enkele probleem in deelproblemen komt overeen met zowel functionele als geografische distributie binnen het probleemdomein.

Nadat er ervaring was opgedaan door middel van de ontwiklkeling en toepassing van de blackboard-shells, ontstond de behoefte om het verkregen inzicht in essentie en toepasbaarheid van de blackboard architectuur nog te vergroten. Daartoe werd een formalisatie ontwikkeld, waarmee bestaande blackboard-architecturen nauweurig en met een uniforme terminologie moesten kunnen worden beschreven. Hoofdstuk 6 bevat deze formalisatie.

Vervolgens werd de formalisatie gebruikt om veertien bestaande architecturen te beschrijven. Deze beschrijvingen zijn te vinden in Appendix $\mathrm{A}$. Onder deze veertien architecturen bewinden zich Blondie, Blondie-Il en Blondie-III. Daarnaast werden elf uit de literatuur welbekende archi- 
tecturen beschreven. Doordat publikaties over deze elf systemen goed toegankelijk zijn, worden deze architecturen vaak als voorbeeld gebruikt. Daardoor vormt de verzameling gekozen ontwerp en implementatie details binnen deze architecturen een grote en betekenisvolle deelverzameling van details binnen alle bestaande blackboard-architecturen.

De formele beschrijvingen uit Appendix A zijn vervolgens gebruikt voor een analyse. Deze analyse was gericht op het vinden van antwoorden op de volgende vier vragen: welke variaties bestaan er in bestaande blackboard-architecturen; hoe kunnen deze variaties worden aangevuld met zinnige, maar nog niet beproefde variaties; wat is de relatie tussen een variant en eigenschappen van de resulterende architectuur; waarin onderscheidt zich een blackboard-architectuur van andere, vergelijkbare programma-architecturen. Antwoorden op deze vragen worden gegeven in Hoofdstuk 7. Deze antwoorden vormen feitelijk de kern van het proefschrift in hun poging tot de essentie van de blackboard-architectuur te komen.

Een belangrijk probleem bij het ontwikkelen van een blackboard-systeem wordt gevormd door het willen vinden van een optimale strategie voor de beslissingseenheid van het systeem: hoe kan die kennisbron worden gekozen voor uitvoering die het meest efficiënt en effectief lijkt op basis van beschikbare informatie. Van nature zijn blackboard-systemen 'datagestuurd': een kennisbron komt voor uitvoering in aanmerking, wanneer een aanknopingspunt voor deze kennisbron verschijnt op het blackboard. Het is echter ook wenselijk dat de geschiktheid van een kennisbron wordt geevalueerd ten aanzien van de te verwachten bijdrage aan het einddoel van het systeem. Deze vorm van 'doel-gericht' probleemoplossen dient geintegreerd te worden met de oorspronkelijke data-gestuurde manier van werken teneinde de wenselijkheid van uitwoering van een kennisbron zo goed mogelijk te kunnen bepalen. Hoewel er enkele voorbeelden bestaan van blackboard-systemen die doelgerichte kennisbronselectie gebruiken, zijn er tot dusverre zijn geen methoden bekend die een echte integratie bewerkstelligen. Dit wordt geillustreerd in Hoofdstuk 8 . Tevens wordt in Hoofdstuk 8 een voorstel beschreven voor een methode op basis van een dynamisch netwerk die wel integratie zou moeten kunnen realiseren. Deze methode is nog niet geverifieerd aan de hand wan een reële toepassing.

Hoofdstuk 9 , tenslotte, bevat conclusies. Dit zijn de algemene conclusies 
die uit het onderzoek kunnen worden getrokken. Meer gedetailleerde conclusies zijn te vinden in de evaluaties aan het eind van de meeste hoofdstukken. Hoofdstuk 9 identificeert tevens enkele onderwerpen die nader onderzoek behoeven. Daarbij wordt vooral aandacht gegeven aan onderzoek dat moet leiden naar de ontwikkeling van een blackboardmethodologie. Een dergelijke methodologie heeft tot doel ondersteuning te bieden bij de ontwerp- en implementatiebeslissingen die genomen moeten worden bij de ontwikkeling van een blackboard-systeem voor een bepaald toepassingsgebied. 


\section{Curriculum Vitae}

Hugo Velthuijsen was born in Driebergen-Rijsenburg (the Netherlands) on April 8, 1960. From 1972 till 1978, he attended the Rijksscholengemeenschap Schoonoord in Zeist. In 1978, he received his Gymnasium- $\beta$ diploma. Next, he studied Mathematics at the University of Amsterdam. In 1986, he received his M.Sc. in Pure Mathematics. Special interests during his studies included Group Theory and Topology. His subsidiary subject was Decision Theory.

Upon completion of his studies, he left the field of Mathematics for an area with better prospects. He joined Dr. Neher Laboratories, the precursor of PTT Research. These laboratories are the research facilities of the Royal PTT Nederland NV, the Dutch mail and telecommunications company. Since then, he has worked in the Artificial Intelligence group of their Computer Science Department. His work consisted primarily of Artificial Intelligence research, ranging over such topics as the blackboard architecture, distributed artificial intelligence, and model-based diagnosis.

Additionally, in 1989, he acted as secretary to the Quality Steering Committee concerned with the introduction of a Total Quality Management programme for PTT Research. Following this experience in a management-oriented task, he decided to pursue a more researchoriented continuation of his career.

Early 1990, he reached agreement with the Rijksuniversiteit Limburg on a plan to complete a $\mathrm{Ph} . \mathrm{D}$. thesis under supervision of Peter Braspenning and Jaap van den Herik, that is based on and extends his previous research in the area of blackboard systems. He also received support from PTT Research to pursue his Ph.D.. A manuscript for this thesis was finished by the end of 1991 . 
Next, he spent a year as a visiting researcher at Bellcore. The purpose of this visit was twofold: helping to substantiate a collaborative agreement between Bellcore and PTT Research and doing research on the application of distributed artificial-intelligence techniques to the management and operations of telecommunications systems. During his stay in the USA, he also completed this thesis. 